\title{
ADVANCING SINGLE MOLECULE FLUORESCENCE MICROSCOPY THROUGH THE STUDY OF DYNAMIC MACROMOLECULES
}

A Dissertation presented to the Faculty of the Graduate School at the University of Missouri-Columbia

In Partial Fulfillment of the Requirements for the Degree:

Doctor of Philosophy in Biochemistry

By: Drew Edwin Menke

Dr. Peter V. Cornish, Dissertation Supervisor

December, 2017 
The undersigned, appointed by the dean of the Graduate School, have examined the dissertation entitled,

\section{ADVANCING SINGLE MOLECULE FLUORESCENCE MICROSCOPY THROUGH THE STUDY OF DYNAMIC MACROMOLECULES}

presented by Drew Menke, a candidate for the degree of doctor of philosophy in Biochemistry, and hereby certify that, in their opinion, it is worth of acceptance.

Professor Peter Cornish

Professor Judy D. Wall

Professor Michael Henzl

Professor Donald Burke 


\section{DEDICATIONS}

I would like to dedicate my dissertation to my parents Peggy and Kevin Menke. You are the most caring parents I could ask for and you continue no matter what, to support, love and nurture me through the challenges I face in life.

To my siblings, Kit, Paul, and Kate Menke, you each hold special places in my heart. From small children playing in mud pits and climbing trees, to fighting as teenagers, to becoming young adults and beyond. Together, you make my life so much better. 


\section{ACKNOWLEDGEMENTS}

This work was carried out in the Department of Biochemistry at the University of Missouri, Columbia.

First and foremost, I would like to thank Peter V. Cornish my advisor. Over the past 7 years we have spent together you have become an amazing friend, mentor and supervisor whose compassion and excitement for science has driven me to become a better scientist. Although I may not have understood 99\% of your movie and sports references, you lightened the lab during the good times and the bad. The ability to walk into your office and talk about my latest science problems, an interesting science question or to just joke around was invaluable. I hope that the work we have done together help you to have a successful career in academia.

I would also like to thank my committee members Judy D. Wall, Michael Henzl, Donald Burke and Gavin M. King for their support throughout my graduate degree.

I would like to thank Jerry Hazelbauer and Linda Randall for providing me with support while completing my degree. Jerry, your calm demeanor and steadfast commitment to the graduate students of this department made a huge impact on me. Linda Randall, the clarity and the empathy you showed toward me will always be remembered.

Donald Burke, you found time even with an already packed schedule to sit and listen and guide me through my degree. Your straightforward frankness has taught me to approach problems with a more objective mind.

Min Pennella, you transformed the way I look at science. Your kindness, persistence, passion, raw talents continue to amaze me.

David W. Emerich, without your support not a single one of my projects would have been possible.

Zenia Norman, we had some great times together and you made graduate school interesting.

To the rest of my lab mates, I would thank all of you for making my time during graduate school what it has been.

Bassem Shebl, we are from different worlds and yet we formed such a tight bond, I will never forget you or the things you have done for me. The encouragement, compassion, and support that you 
showed me throughout my graduate degree were invaluable. You kept me going even when I thought I could not and there are not enough words in this world for me to try to describe what you mean to me. It has been a pleasure working with you and I wish you all the luck and success that this world can offer. I know for a fact that you will continue to accomplish great things. 


\section{TABLE OF CONTENTS}

ACKNOWLEDGEMENTS ................................................................................................ii

LIST OF ILLUSTRATIONS ........................................................................................vii

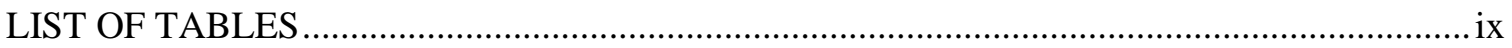

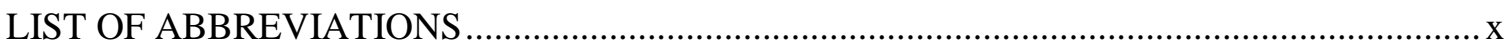

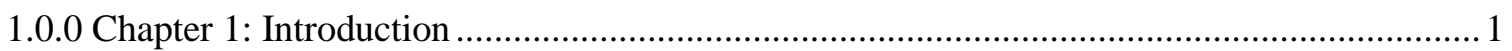

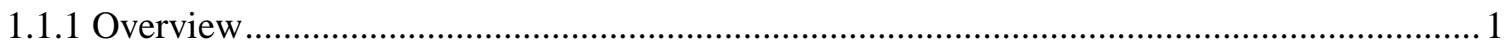

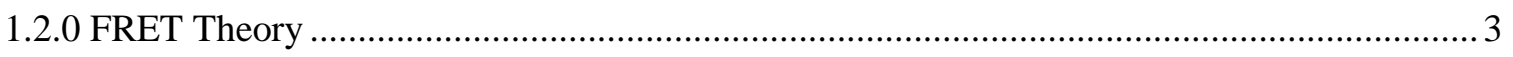

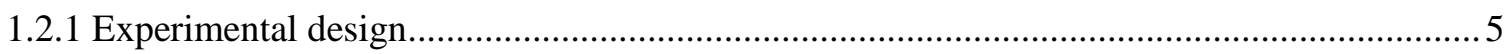

1.3.0 Microscopy and technology …………………………….....................................

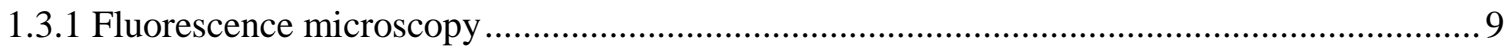

1.3.2 Single molecule fluorescence resonance energy transfer (smFRET) ……........................... 10

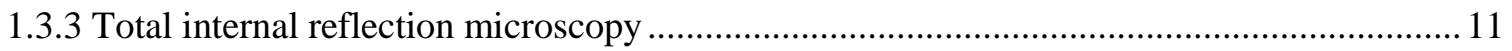

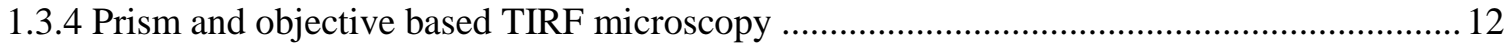

1.3.5 Methods of selective fluorescence observations ..............................................................13

1.4.0 Ribosome structure …………………………………………………………………... 14

1.4.1 X-ray crystallography and the ribosome ……………………………………………..... 14

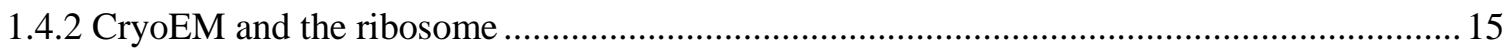

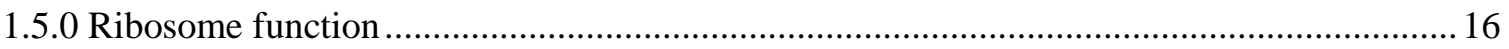

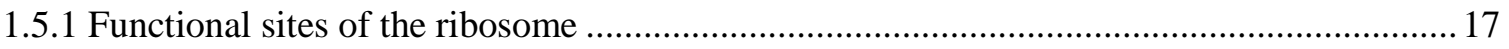

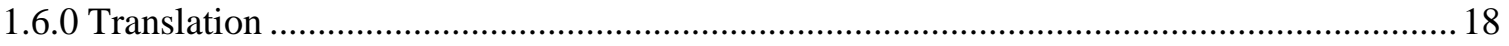

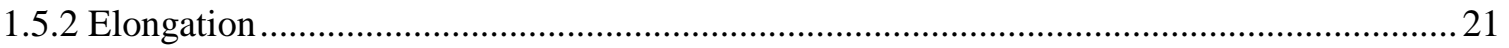

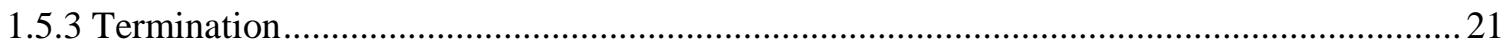

1.6.0 Using smFRET to examine large scale dynamic motions of the ribosome …………….......22

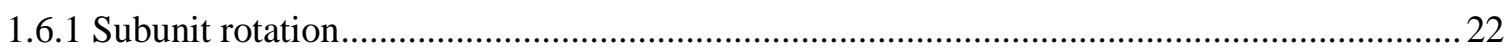

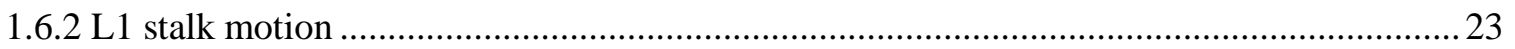




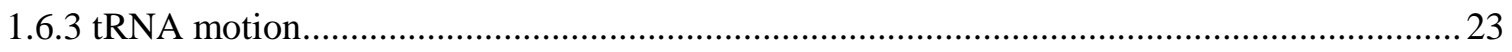

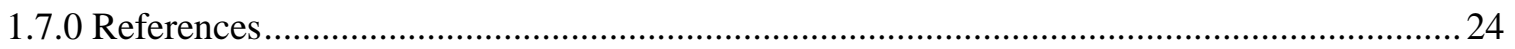

2.0.0 Chapter 2: Fluorescence enhancement from nano-gap embedded plasmonic gratings by a

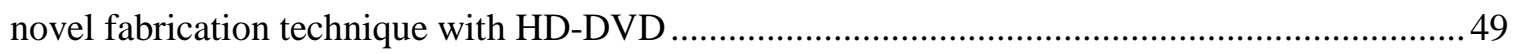

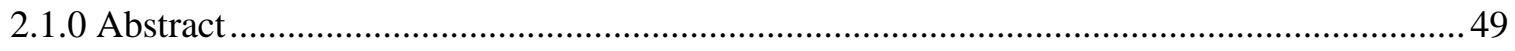

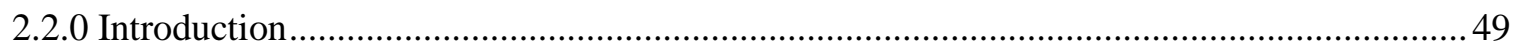

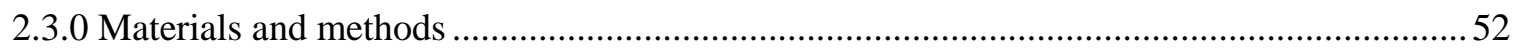

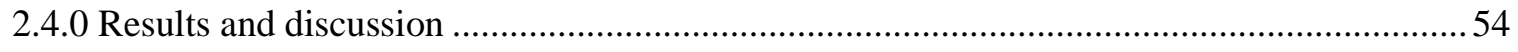

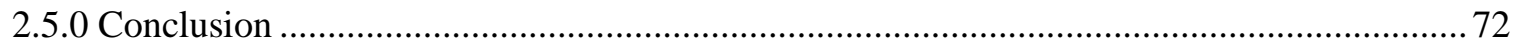

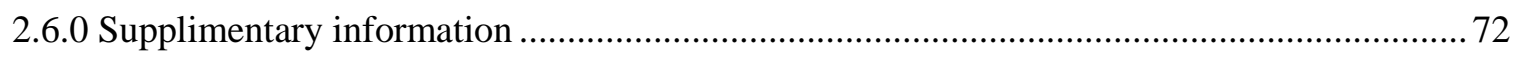

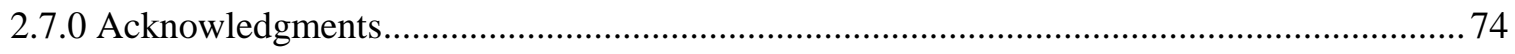

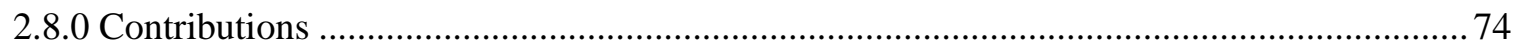

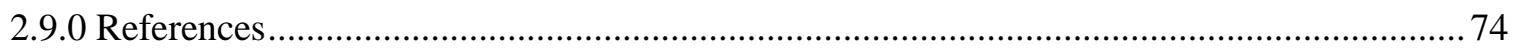

3.0.0 Chapter 3: Single Molecule Oscillations of an RNA/DNA Duplex in a Plasmonic

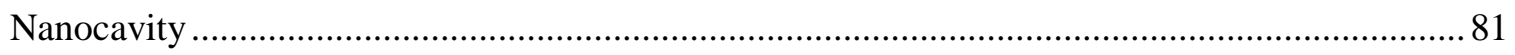

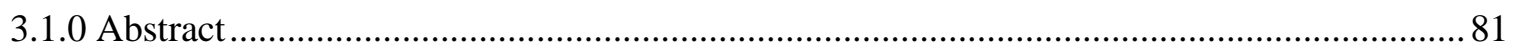

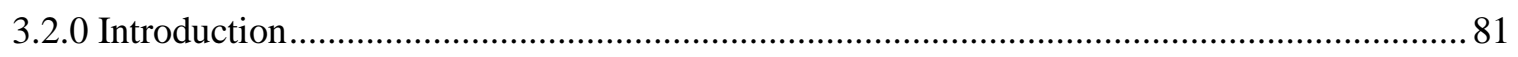

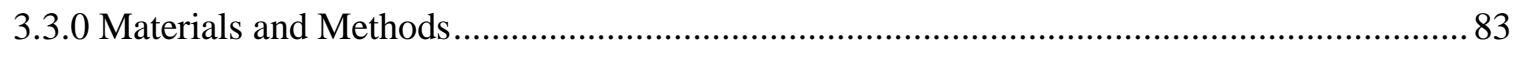

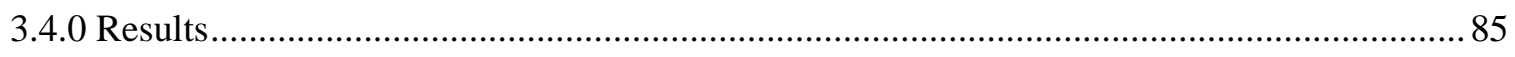

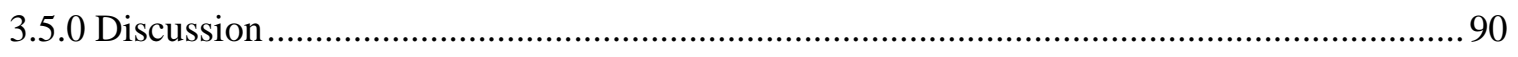

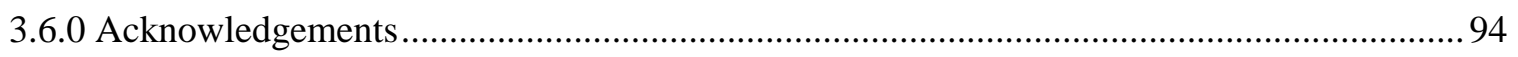

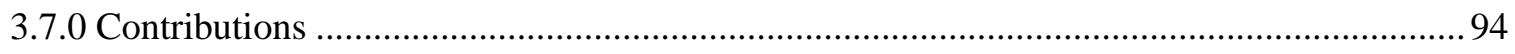

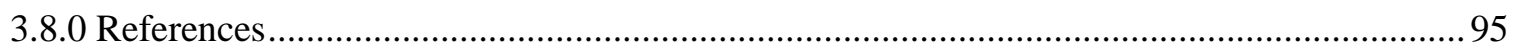

4.0.0 Chapter 4: Preparation of Ribosomes for smFRET Studies: A Simplified Approach ..........98

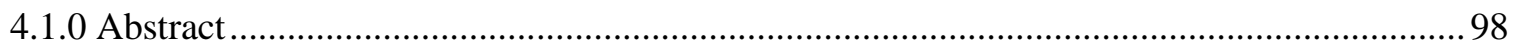

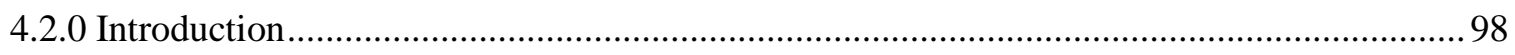




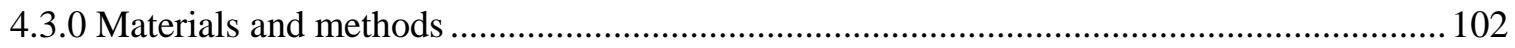

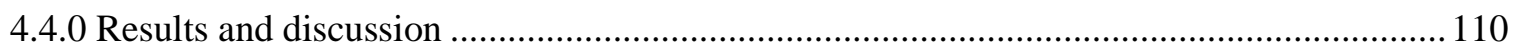

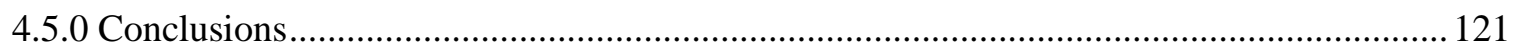

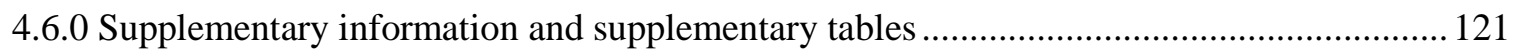

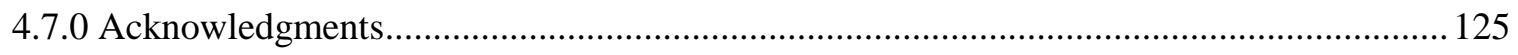

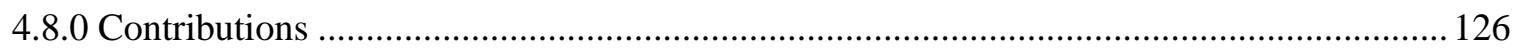

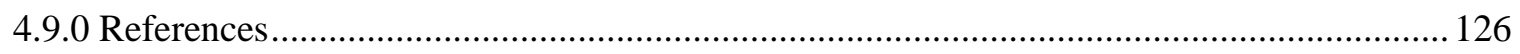

5.0.0 Chapter 5: Using smFRET to dissect the L1 stalk-tRNA interaction ................................ 131

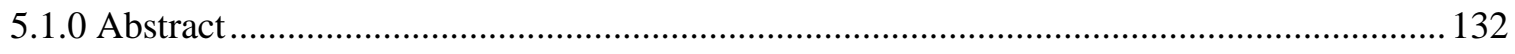

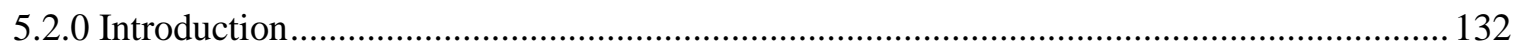

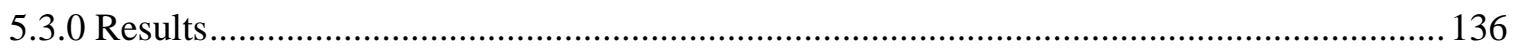

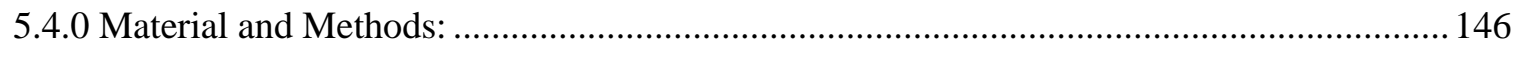

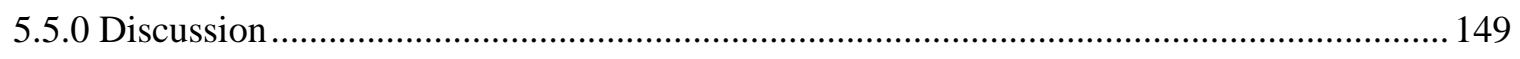

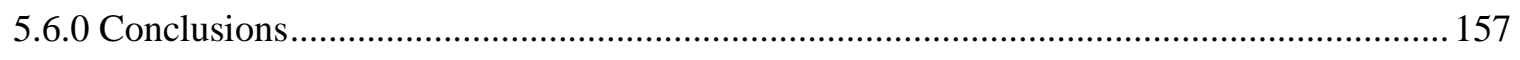

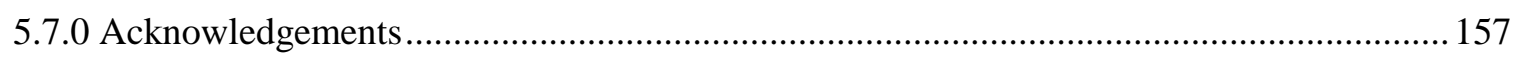

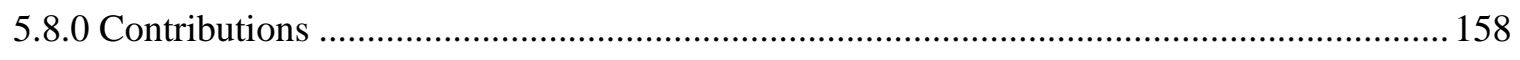

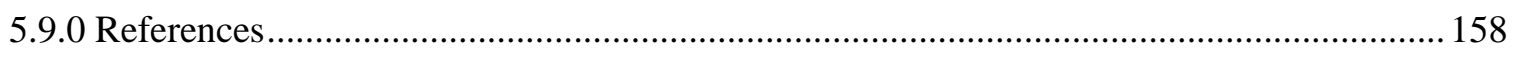

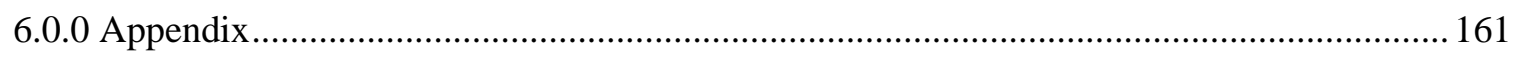

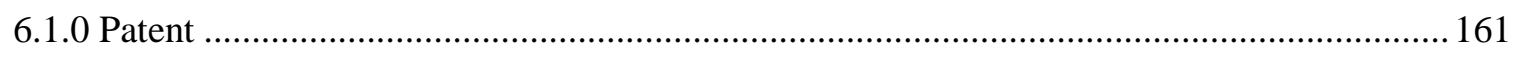

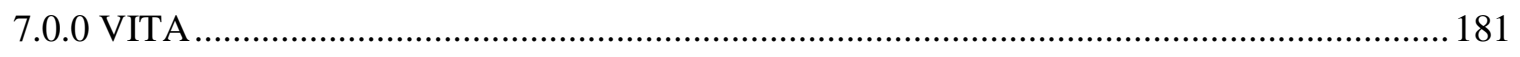




\section{LIST OF ILLUSTRATIONS}

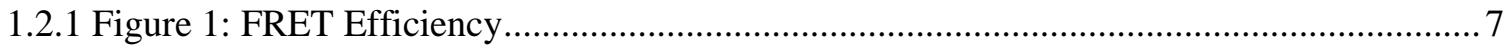

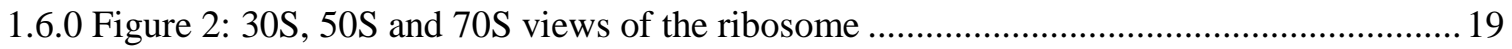

1.6.0 Figure 3: Model of translation: Initiation, elongation, termination and recycling ................20

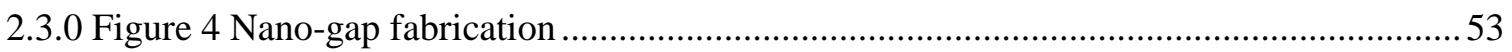

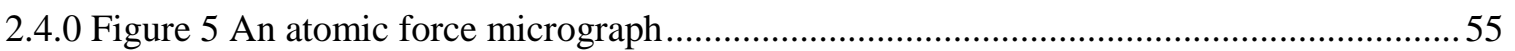

2.4.0 Figure 6 A top-view SEM image of the nano-gap embedded in the silver gratings .............56

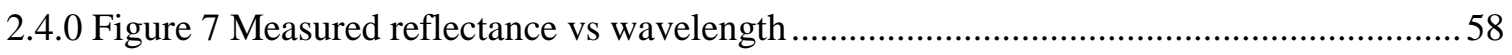

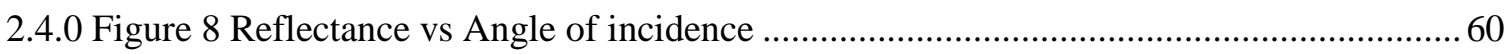

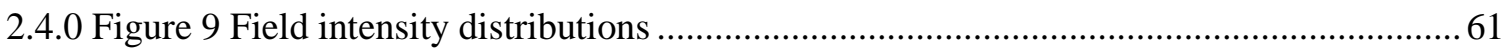

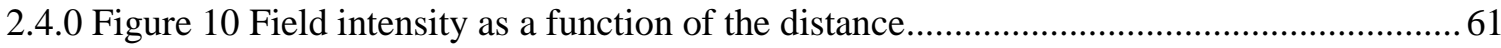

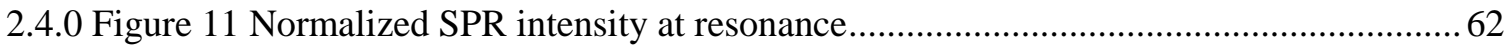

2.4.0 Figure 12 Contour profile of the nano-gap embedded in silver gratings ............................63

2.4.0 Figure 13 Field intensity distributions at the air-silver interface ...................................... 64

2.4.0 Figure 14 The excitation and emission spectra of Rhodamine 590 (R6G) ........................66

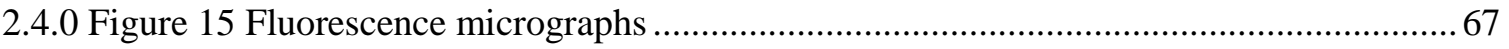

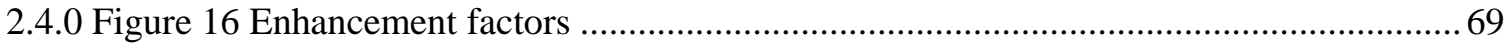

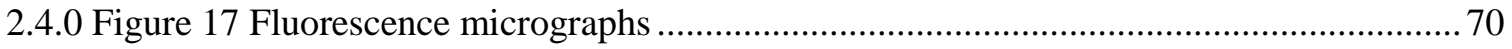

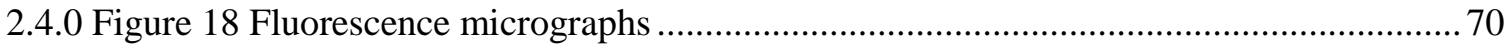

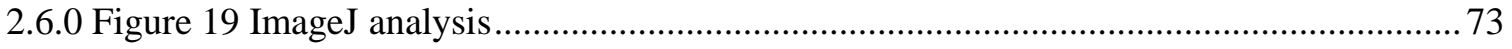

2.6.0 Figure 20 Reflectance spectra for silver gratings............................................................... 74

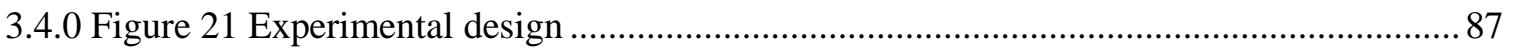

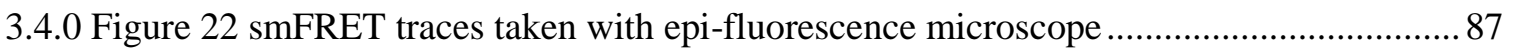

3.5.0 Figure 23 Fluorophore intensity oscillation frequency ............................................... 91

3.5.0 Figure 24 Schematic of the ion concentration distribution and duplex behavior..................91

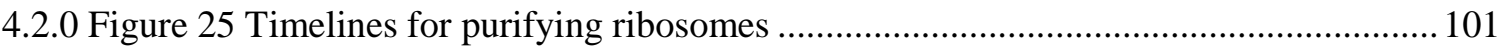




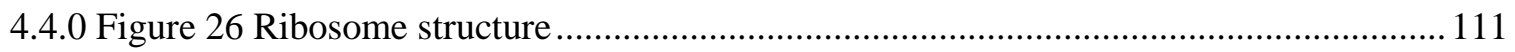

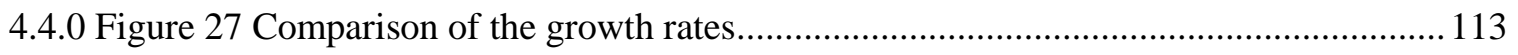

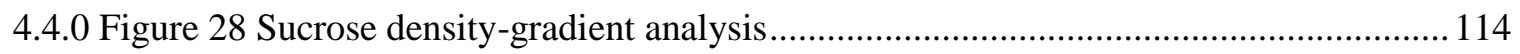

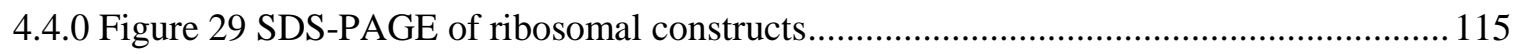

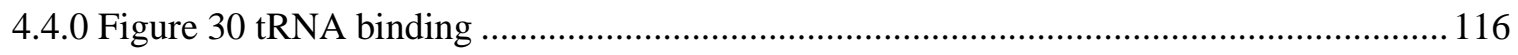

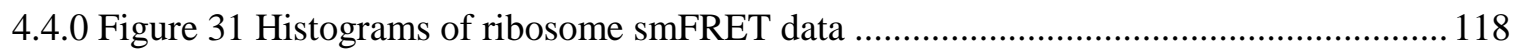

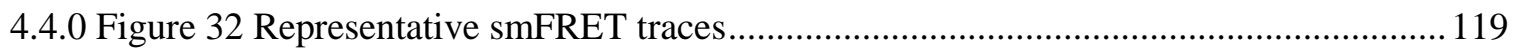

4.6.0 Figure 33 Sucrose density-gradient analysis.............................................................. 123

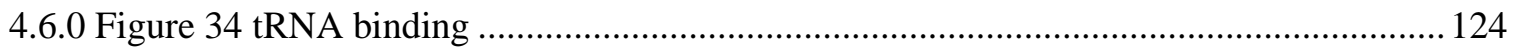

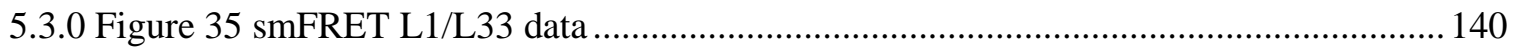

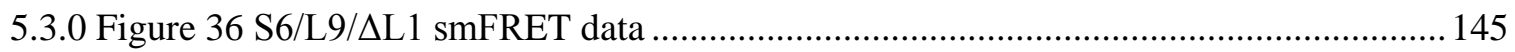




\section{LIST OF TABLES}

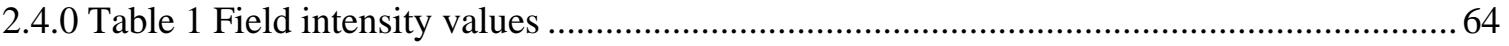

2.6.0 Table 2 Enhancement factors and average intensity values.......................................... 73

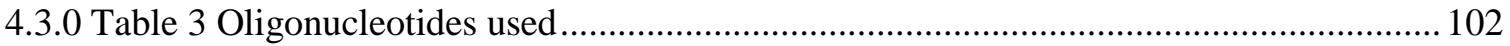

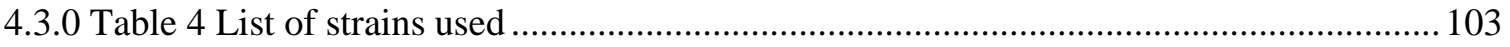

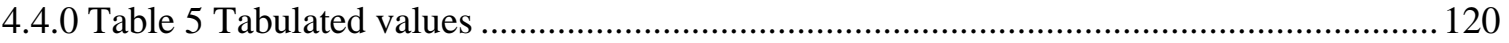

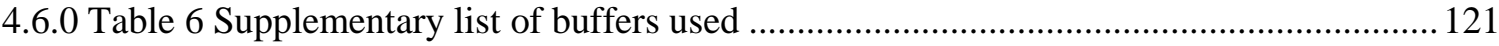

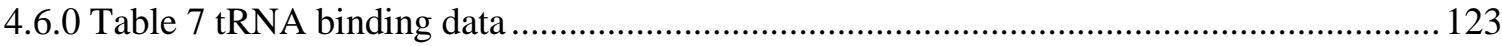

5.3.0 Table 8 Calculates values for the L1/L33 smFRET data ................................................ 141

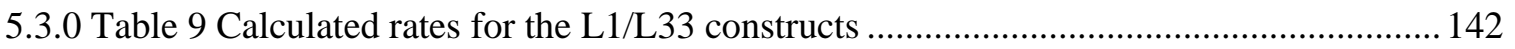

5.3.0 Table 10 Calculates values for the S6/L9/AL1 smFRET data ............................................ 146 


\section{LIST OF ABBREVIATIONS}

O6-alkylguanine-DNA alkyltransferase (AGT), amino acyl site (A-site), arbitrary units (au), $\beta$ mercaptoethanol ( $\beta \mathrm{ME})$, cartilage hair hypoplasia $(\mathrm{CHH})$, chloramphenicol (cam), continuous wave $(\mathrm{CW})$, Cryo-electron microscopy (CryoEM), C-terminal domain (CTD), Diamond-Blackfan anemia (DBA), Xlinked dyskeratosis congenita (DKC), electron multiplying charged coupled device (EMCCD), electron paramagnetic resonance (EPR), elongation factor G (EF-G), elongation factor Tu (EF-Tu), exit site (E-site), fast Fourier transform (FFT), finite difference time domain (FDTD), grating coupled surface plasmon resonance (GC-SPR), high-definition (HD), initiation factors (IF), kanamycin (kan), localized surface plasmons (LPs), Mega Dalton (MDa), messenger ribonucleic acid (mRNA), milliseconds (ms), N-terminal domain (NTD), peptidyl site (P-site), peptidyl transferase center (PTC), perfect electric conductor (PEC), perfectly matched layer (PML), photo multiplier tubes (PMT), polydimethylsiloxane (PDMS), polymethylsilsesquioxane (PMSSQ), power spectral density (PSD), protein induced fluorescence enhancement (PIFE), reactive ion-etching (RIE), resonance energy transfer (RET), Rhodamine 590 (R6G), ribosomal RNA (rRNA), ribosome recycling factor (RRF), Schwachman-Diamond syndrome (SDS), scientific CMOS (sCMOS), Shine-Dalgarno sequence (SD), signal-to-noise ratio (SNR), single-molecule (SM), single molecule fluorescence resonance energy transfer (smFRET), Small Angle Neutron Scattering (SANS), surface enhance Raman spectroscopy (SERS), surface plasmon-coupled emission (SPCE),surface plasmon polaritons (SPPs), surface plasmon resonance (SPR), total internal reflection (TIR), total internal reflection microscopy (TIRFM), transmission electron microscopy (TEM), transverse electric (TE), transverse magnetic (TM), Treacher Collins syndrome (TCS), tris (2-carboxyethyl) phosphine (TCEP), and variable angle spectroscopic ellipsometer (VASE). 


\subsubsection{Chapter 1: Introduction}

\subsubsection{Overview}

Translation is the process by which the ribosome synthesizes proteins and is essential to all living organisms. Understanding the mechanism by which protein synthesis occurs has been a focus of biologists for many years. Protein synthesis is an important part of the central dogma of life which states that DNA, the genetic material responsible for the storage of information, must first be made into RNA through a process called transcription, which can then made into protein by translation. Proteins provide important functions inside and outside the cell, including, the ability to catalyze reactions, signal information and provide structural support.

The synthesis of protein by the ribosome is important because the mutation of genes coding for ribosomal RNA or proteins can lead to various deleterious phenotypes. In humans, defective ribosomes diseases are classified as ribosomeopathies some of which include Diamond-Blackfan anemia (DBA), Schwachman-Diamond syndrome (SDS), X-linked dyskeratosis congenita (DKC), cartilage hair hypoplasia (CHH), and Treacher Collins syndrome (TCS). These diseases typically include bone marrow failure and/or skeletal defects. Not only have some of these genetic defects been linked to an increased risk of getting cancer, but ribosomes play an important role in the cell because they have been implicated in almost every major biological process: apoptosis, DNA repair, regulation and development, cell migration and cell cycle control [1]. Studying eukaryotic ribosomes is currently challenging, however, a large amount of work has already been completed on the study of bacterial ribosomes, which share many of the same structural motifs and core processes. One day, the information gained from studying bacterial ribosomes may be applied to help understand eukaryotic ribosomes as well.

The ribosome and the process of translation have been studied by various techniques since its discovery by George Palade in 1955 by electron microscopy [2]. Over time, scientists have steadily pieced together the mechanism of translation through extensive research. One critically important field, structural biology, has been instrumental in revolutionizing research on translation. At the turn of the $20^{\text {th }}$ century, structural biology became a much more valuable tool and has seen an impressive amount of progress in obtaining X-ray crystallographic and Cryo-electron microscopy (CryoEM) structures of the ribosome and many other complex biomolecules. In 2009, Ada Yonath, Venkatraman Ramakrishnan and Thomas Steitz 
were awarded the Noble Prize in Chemistry for their pioneering work towards structure determination of the ribosome by X-ray crystallography. Ada Yonath pioneered some of the first work with growth and production of crystals suitable for X-ray diffraction. [3-7]. High resolution X-ray crystallographic data has given scientists working on translation an unprecedented look at the detailed structure of the ribosome. The combination of structural data with other methods has aided in more detailed investigation of the mechanism of translation and also the development of treatments and/or cures for modern day ribosome linked ailments.

Single molecule fluorescence resonance energy transfer (smFRET) is one tool that has been used extensively to study the mechanism of bacterial translation. SmFRET provides information about the dynamics of molecules and is often used in conjunction with structural data to address specific mechanistic questions. In the case of the ribosome, smFRET has been used to understand how large-scale conformational motions of the ribosome function and how they are influenced by various translation factors during translation. Today, researchers recognize that the ribosome is a highly dynamic and interconnected macromolecular complex, where large-scale conformational dynamics play an important role during translation. Subunit rotation, which is the rotation of the 30S and 50S subunits is one of the largest dynamic motions of the ribosome and has been studied using FRET and smFRET [8, 9]. SmFRET has also been used to examine the fluctuations of tRNA within the ribosome during translation, and how these motions facilitate the translocation of tRNA though specific tRNA binding sites within the ribosome [10-12]. The L1 stalk, an rRNA protrusion bound to ribosomal protein L1, has been shown by smFRET to participate in stabilization of tRNA on the ribosome and is thought to facilitate removal of exit site tRNA [13, 14]. Taken together, visualization of these motions has contributed to a greater understanding of the various stages of translation including initiation[15], elongation [16], and termination/recycling[17]. Other more specialized processes of translation are beginning to be investigated, including movement of the 30S's head [18], ribosomal frameshifting [19, 20], and regulation of protein synthesis [21].These studies have been invaluable and have given researchers a much better understanding of translation, but there is still a large amount of information we do not know about the mechanism of translation.

In order to be able to better understand the ribosomes, we have developed a two-pronged approach: (1) Advance and improve fluorescence observation of samples and (2) improve upon already 
existing methods for generation of ribosome samples for use with smFRET experiments. The need for both of these technologies is evident. The high costs associated with this instrumentation have limited the availability of these technologies, and there is an ever-growing need for the collection of high resolution data at a low cost with high signal-to-noise using fluorescence methods.

We have developed one such method for the collection of high-resolution data of fluorescence signal using plasmonic gratings, which can be used with conventional fluorescence microscopy. This technology has been tested on a biologically relevant sample composed of a RNA/DNA hybrid (Chapter 2 and 3). Also, we have developed a method for the generation of ribosome constructs for smFRET studies that is cheaper, quicker and results in a sample that is the same, if not better, than traditional methods (Chapter 4 and 5). Finally, we applied these sample preparation methods to the production of ribosome constructs that are designed to examine the interactions of the L1 stalk with tRNA and how these interactions can affect subunit rotation. As mentioned, the development of techniques in microscopy and the generation of samples for smFRET investigation is an integral part of the strategy we took in our work and a common theme throughout.

\subsubsection{FRET Theory}

One of the main methods we have used during our studies was Fluorescence resonance energy transfer or Förster resonance energy transfer, which was named after Theodor Förster who discovered it in 1946. FRET is a process by which an excited fluorophore of higher energy and shorter wavelength is able to transfer energy in a distance dependent manner to excite another fluorophore of the same or longer wavelength and with lesser energy. Modern biology has embraced this technique as a method for observation dynamics of macromolecules though the use of single smFRET. SmFRET has helped to establish a better understanding of the dynamics of complex macromolecules not previously attainable. The FRET process is defined by a set of equations which include a number of internal properties inherent to the fluorescent dye used and surrounding environment, which are important considerations when designing and performing smFRET experiments. A more detailed examination of FRET and its theory can be found in the following references $[22,23]$. 
FRET or even more simply Resonance energy transfer (RET) is governed by the following equation and variable. RET between a single donor and acceptor can be defined in terms of their distance apart and rate of energy transfer through the following equation:

$$
K_{T}(r)=\frac{Q_{D} k^{2}}{\tau_{D} r^{6}}\left(\frac{9000(\ln 10)}{128 \pi^{5} N n^{4}}\right) \int_{0}^{\infty} F_{D}(\lambda) \varepsilon_{A}(\lambda) \lambda^{4} d \lambda
$$

Where the rate of energy transfer $(r)$ is the distance between the donor and acceptor as is determined by: $Q_{D}$ which is the quantum yield of the donor without the acceptor present, $\mathrm{n}$ is the refractive index of the medium the sample is within, $\mathrm{N}$ is Avogadro's number, $\mathrm{r}$ is the distance between the donor and acceptor, and $\tau_{\mathrm{D}}$ is the lifetime of the donor without the acceptor present. For smFRET base experiments, which are commonly performed on quartz slides in a biologically relevant solution the refractive index (n) is assumed to be 1.4. The corrected fluorescence intensity $F_{D}(\lambda)$ is the intensity of the donor wavelength range $\lambda$ to $\lambda+$ $\Delta \lambda$ with the total intensity normalized. The factor $\varepsilon_{\mathrm{A}}(\lambda)$ is the extinction coefficient of the acceptor at $\lambda$, which is typically reported in units of $\mathrm{M}^{-1} \mathrm{~cm}^{-1}$. The factor $\kappa^{2}$ is describes the relative orientation in space of the transition dipoles of the donor and acceptor. It is common for $\kappa^{2}$ to be assumed to be $2 / 3$, which is the approximation for random averaging of the donor and acceptor. A $\kappa^{2}$ equal to 0 represents the rigid orientation of the dyes dipole perpendicular, $a \kappa^{2}$ of 1 would represents the dye dipoles as parallel and a $\kappa^{2}$ of 4 has the dipole of the dye in a straight line. It is not uncommon to experience difficulties associated with $\kappa^{2}$ when designing and performing experiments because of confinement and/or interactions between the hydrophobic ringed structure of some dyes and biological structures.

The previous equation can be rewritten in terms of the distance between the donor and acceptor:

$$
R_{0}^{6}=\left(\frac{9000(\ln 10) k^{2} Q_{D}}{128 \pi^{5} N n^{4}}\right) \int_{0}^{\infty} F_{D}(\lambda) \varepsilon_{A}(\lambda) \lambda^{4} d \lambda
$$

The Transfer rate from donor to acceptor can be rewritten as:

$$
\text { Transfer Rate }=k_{T}(r)=\frac{1}{\tau_{D}}\left(\frac{R_{0}}{r}\right)^{6}
$$

Where $\mathrm{k}_{\mathrm{T}}(\mathrm{r})$, is the transfer rate that occurs between a donor and acceptor fluorophore at a defined rate that is defined by $\tau_{\mathrm{D}}$ which is the decay time of the donor without the acceptor present, $\mathrm{R}_{0}$ is the Förster distance and $\mathrm{r}$ is the distance between the donor and acceptor fluorophores. The $\mathrm{R}_{0}$ is the distance at which $50 \%$ of the energy from the donor is being transferred to the acceptor. RET is based on an inverse sixth 
power law which make is strongly dependent on the distance between the donor and acceptor molecules [24].

The efficiency of energy transfer (E) during FRET is the fraction of photons absorbed by the donor which are ultimately transferred to the acceptor and is the ratio of the transfer rate by the total decay rate of the donor when the acceptor is present:

$$
E=\frac{k_{t}(r)}{\tau_{D}^{-1}+k_{t}(r)}
$$

This equation can be rearranged using $k T(r)=\tau D-1\left(R_{0} / r\right)^{6}$ from the previous equation to define $\mathrm{E}$ which is the fractions of photons absorbed by the donor and subsequently transferred to the acceptor, $\mathrm{R}_{0}{ }^{6}$ is the Förster distance at which $50 \%$ of energy transfer occurs and $\mathrm{R}^{6}$ is the distance between the donor and acceptor.

$$
E=\frac{R_{0}^{6}}{R^{6}+R_{0}^{6}}
$$

These equations demonstrate that the transfer of energy is strongly dependent on the distance between the donor and acceptor when close to the $\mathrm{R}_{0}$. The efficiency of energy quickly increases to 1.0 when the donor and acceptor are in close proximity and decays quickly to 0 over a certain limit, often near $10 \mathrm{~nm}$.

FRET is influenced by a number of factors including, the photo physical properties of the dye, solution environment, and rotational orientation. This makes it critical to take into account both the biological sample and dyes that will be used for experiments.

\subsubsection{Experimental design}

When designing an experiment it is important to consider the dye pair you will use, the labeling sites, and the methods for generation of the sample. The choice of dyes to be used for smFRET is essential to the generation of successful smFRET construct. Cyanine dyes are widely used because of their similar quantum yields and detection efficiencies as well as their good photo stability and suitable properties for use with biological samples [25]. They come in a large range of fluorescent spectra from $\sim 450-750 \mathrm{~nm}$, are easily detectible and have a good quantum yield which make them suitable for FRET. Cyanine dyes come in a number of forms including a non-sulfonated or sulfonated form, which is less hydrophobic, can be less prone to interact with your biological sample and precipitate in aqueous buffers. Conjugation of trolox and 
other triplet state quenchers to cyanine dyes has been shown to significantly increase time spent in the fluorescent state and prevent triplet state and blinking [26]. There are also alternatives to using Cyanine dyes including Atto and Alexa dyes. Atto and Alexa dyes can be excellent choices for FRET dye pairs because of their similar properties to cyanine dyes [24].

Constructs design should take advantage of the most sensitive region of the dye pairs FRET efficiency curve. This is the linear region of the FRET efficiency curve and for Cy3 and Cy5 it is between 2-8 nanometers. It is situated around the $\mathrm{R}_{0}$, which is the point at which $50 \%$ of energy transfer from donor to acceptor occurs, allowing for the detection of small motions of a biomolecule. If the motion you are trying to examine for the construct is shifted closer or further away from the $\mathrm{R}_{0}$ for a specific dye pair it may be preferable to work with an alternative set of dye pairs (Figure 1). The disadvantage of choosing a dye pair not properly suited for your experimental design is insensitivity and lack of resolution to the dynamic motion being examined. The labeling positions chosen are critical to the observation of the dynamics and prevention of problems with respect to the orientation factor of the dyes [27].

Dye effects are a persistent problem when performing smFRET experiments, however there have been a number of methods developed to mitigate their influence on the data. The conversion of a fluorescent molecule to the triplet state, which is seen in the data as blinking, and the premature conversion to a dark state called, photo-bleaching, as a result of its reaction with oxygen. These processes can be somewhat controlled through the use of triplet state quenches like TROLOX and BME, and enzymatic oxygen scavenging systems like glucose oxidase and catalase. The development of alternative systems like protocatechuic acid/protocatechuate-3,4-dioxygenase have resulted in advantages over traditional methods like $\mathrm{pH}$ stability as well in addition to better control over dissolved oxygen in solution. 


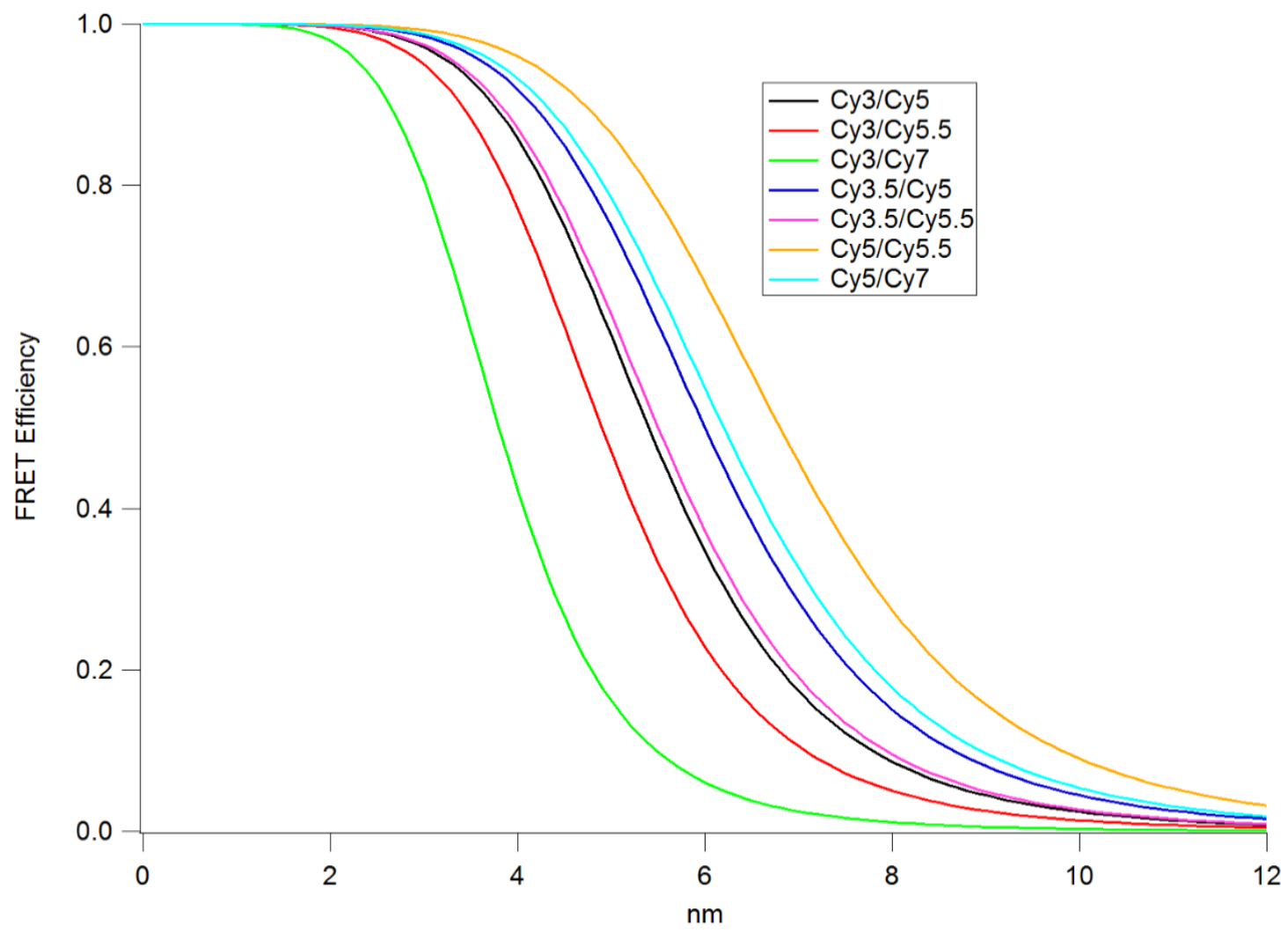

\subsubsection{Figure 1: FRET Efficiency}

A graph with the use of known $\mathrm{R}_{0}$ values for the following dye pairs: Cy3/Cy5, Сy3/Cy5.5, Cy3/Cy7, Cy3.5/Cy5, Cy3.5/Cy5.5 Cy5/Cy5.5 and Cy5/Cy7. Note the linear range, change in position with respect to $\mathrm{nm}$ and diversity of the various FRET dyes pairs displayed [28] [29].

A number of options exist for the conjugation of fluorescent dyes to a biomolecule with the existing fluorescent probes and different systems. There is a large diversity of compatible, spectrally separated fluorescent probes that can be conjugated to DNA, RNA and protein through a number of different methodologies. Some general choices for linking to different biomolecules include alkyne (ethynyl), azide, NHS ester, maleimide and hydrazide chemistry. Thiol-maleimide conjugation chemistry is a common choice for in vitro protein labeling that contains only a single cysteine. In addition to chemical labeling, there exist alternative methods one of which takes advantage of the ability to reprogram specific codons to encode for un-natural amino acids. This method has been employed in the reprograming of specific codons in Escherichia coli to incorporate an unnatural amino acid into a protein at a specific position [30,31]. The purified components containing the specific unnatural amino acid can then be labeled 
through conventional methods. It was previously demonstrated as a method for dual labeling of a protein for use with smFRET [32]. The same methodology has also been expanded for use in Eukaryotic systems as well $[33,34]$.

Site specific labeling of proteins can also be accomplished through the use of small unique protein tags, two examples of which are SNAP and CLIP. These systems are used to covalently link a small labeled protein onto the protein of interest at a specific protein sequence. SNAP-tag is derived from the human DNA repair protein, O6-alkylguanine-DNA alkyltransferase (AGT), and can be covalently linked to O6benzylguanine derivatives bearing a chemical probe. CLIP-tag, an AGT-based tag which reacts with O2benzylcytosine derivatives. SNAP and CLIP were some of the first derived proteins to allow for labeling of this kind and [35] because SNAP-tag and CLIP-tag possess orthogonal substrate specificities, SNAP and CLIP fusion proteins can be used simultaneously with different molecular probes in living cells [36]. Depending on the design and implementation of this labeling methodology these tags should have little to no effect on bioactivity [11].

Protein induced fluorescence enhancement (PIFE) occurs when a protein is able to bind to a section of nucleic acid in the proximity of a fluorescent probe. The protein prevents the formation of cis isomerization which results in an increase in fluorescence intensity [37]. It is extremely important that you take into account the advantages and limitations of FRET when designing and executing an experiment [24, 38-40].

\subsubsection{Microscopy and technology}

Microscopy includes a number of different techniques used to obtain information about a sample. Modern microscopes can use light, electrons or even force to probe samples in solution or on a surface and have proved crucial to the investigation of numerous research topics. The ultimate goal of microscopy is to observe a sample in its native state, in vivo. The development of new technologies that are able to overcome conventional limitations have provided researchers with new and exciting opportunities for the future. In recent history, microscopy has advanced to the point where it has been used to detect disease markers and continues to be used to ask questions about biology, materials and life on other planets. 


\subsubsection{Fluorescence microscopy}

Fluorescence microscopy, unlike optical microscopy, allows for the selective excitation and observation of emission light of one or many spectrally separate fluorescent probes that can be specifically attached to target molecules. The emitted light by the fluorophores is separated from the excitation light before imaging using a set of optical filters. The advantage of fluorescence microscopy techniques is that they allow for detailed localization of fluorescent probes, which can be used to aid in structural and functional understanding of the cell.

Epifluorescence microscopy utilizes the microscope's objective lenses to act as both the illumination condenser and the collector of fluorescent light and allows for direct observation of living organisms by excitation of fluorescent molecules incorporated into the organism or through fixation and fluorescent labeling. In the past, fluorophores have been used to label specific structures or track a molecule within the cell. Two main methods exist for the labeling of a sample. This includes the attachment of a chemically synthesized fluorophore in vitro or the use of the cellular machinery and genes that code for fluorescent proteins or RNA which are joined in-vivo. In-vivo labeling methods can be very effective with high specificity and being relatively non-invasive method. Fluorescence microscopy users can take advantage of multiple fluorescent labels of various wavelengths in order to localize multiple signals within the cell to track several different processes simultaneously. One of the main disadvantages when using epifluorescence microscopy can be the low single-to-noise caused by scattered light, which decreases the clarity of the image by increasing background fluorescence. One major advantage is the ease of operation and the ability to observe samples from one side. The disadvantages of epifluorescence microscopy are the use of photo-bleachable probes, bulk imaging and diffraction limited images. A number of techniques like confocal microscopy have been developed to address diffraction limited images, high background and sensitivity of epifluorescence microscopy.

Confocal microscopy, an advanced method for the observation of fluorescence signals, can be broken up into two main techniques, laser scanning confocal microscopy and spinning disk confocal microscopy. Both techniques rely on the rejection of out of focus light to obtain high quality images of fluorescently labeled samples. The primarily advantage of confocal imaging is its use in live cell imaging, 
predominantly in the cases having to do with the localization of specific cellular components, or it can be used to examine how a cell responds to a specific process.

Laser scanning confocal microscopy, a variation of confocal microscopy, relies on the use of a raster scan methodology for excitation and imaging. A pair of pinhole apertures are used to block out-offocus light, which results in improved image quality and signal-to-noise. A set of movable mirrors are used to scan the sample and create the image. This method of imaging can be very time consuming to acquire a data set because the imaging speed is limited to the collection of a single point of your image at a time. It does however have a high sensitivity and ability to reach high time resolutions because of use of photo multiplier tubes (PMT) for recording images. It was one of the first techniques to be used to observe smFRET [24].

Spinning-disk confocal microscopy, a much higher throughput method allows for imaging of a large field of view and is often used for live cell imaging. Spinning disk confocal instruments, like Yokogawa units, are equipped with a pair of patterned disks. One disk contains a set of holes designed to reject out of focus light and the other a set of micro lenses inserted into the spinning disk that focus the light for imaging. It uses the same principles and concepts of laser confocal, however, over a much larger area with the use of spinning disks with precisely positioned pin holes [41]. Confocal microscopy and other fluorescence microscopy techniques have proven to be valuable tools in the study of structure-function relationships and in understanding structural dynamic rearrangements. Biochemistry, biophysics and structural biology continue to use many of these techniques to address ever more detailed scientific problems and further improvements will help to enhance and improve upon existing methods. An alternative method for excitation of surface-bound molecules is prism and objective based total internal reflection microscopy, which can produce similar levels of signal-to-noise but with higher throughput.

\subsubsection{Single molecule fluorescence resonance energy transfer (smFRET)}

SmFRET was first established by Ha et al. in 1996 [42]. Since then, smFRET has become a powerful technique that has evolved into a valuable resource used to dissect the molecular interactions and conformational dynamics of macromolecules. Structural data have been extremely helpful in the design of smFRET experiments and have provided information that aid in the design and placement fluorescent dye on a construct. smFRET experiments are able to observe dynamics down to several milliseconds (ms) with 
a conventional EMCCD setup. However, the use of PMT can extend the range into the ns range. SmFRET is typically performed using prism TIRF because of its high signal-to-noise ratio, however, a correctly configured laser scanning confocal microscope can be used as well.

FRET is the non-radiative transfer of energy that can take place between two fluorescent dyes, called the donor and the acceptor, and are in close proximity: 0-10 nanometers [43]. With the most sensitivity for FRET for Cy3 and Cy5, two commonly used fluorescent dyes, between 2-8 nanometers. During analysis FRET is calculated by comparing the intensity of donor channel to the acceptor channels over time allowing for the calculation of the amount of energy that has been exchanged. Where $\mathrm{A}$ is the intensity of the acceptor and D is the donor and FRET is the calculate FRET that has occurred between the two dye.

$$
\mathrm{A} /(\mathrm{D}+\mathrm{A})=\mathrm{FRET}
$$

Data is collected as a series of movies which are further analyzed to determine how the molecule interacts. SmFRET represents a challenging, but rewarding, technique that, when correctly applied, provides one of the few methods for observation of real time dynamics. Two-color smFRET, represents the most commonly applied methodology of smFRET and has typically been used to examine a single dynamic process at a time. SmFRET has been applied to numerous systems, including RNA and DNA, providing detailed dynamics information and helps further our understanding of the mechanism of interaction for many biomolecules.

\subsubsection{Total internal reflection microscopy}

Total internal reflection (TIRF) is a method of selectively exciting surface-bound molecules to limit the excitation volume to a depth of only a few hundred nanometers from the coverslip or slide surface. Typically, a laser is employed as the light source of choice because of its small bandwidth and tightly collimated beam. Light refracted off of the interface between two materials of different refractive index at what is called the critical angle and results in the generation of an evanescent wave at the interface between the two surfaces. The advantage of TIRF over other methods like epifluorescence microscopy is the reduced excitation volume, which provides for a reduction in the background fluorescence and helps to enhance the signal-to-noise ratio. TIRF microscopy has a broad range of applications, including sensing and use fluorescence microscopy techniques, like smFRET and super resolution imaging [41]. TIRF occurs 
when light passes from an interface of high refractive index to one of lower refractive index. If the incident light encounters a difference in refractive index that is greater than the critical angle the light will undergo what is called total internal reflection. This means that the incident light that encounters the surface will be reflected because of the difference in surface refractive indices and will not be allowed to enter the material of lower refractive index. At the point where these two surfaces of differing refractive indices meet is where there will be the generation of an electromagnetic field called an evanescent wave. This evanescent wave generated at the point of TIRF is what is used to excite samples using this technique. The evanescent wave created by TIRF decays exponentially the further you get away from the two surfaces with a range of $\sim 30-300 \mathrm{~nm}$. TIRF does not occur as a sudden effect but slowly as you approach the critical angle and in a continuous transition from transmittance to reflectance. As you approach the critical angle, the amount of transmitted light is reduced and reflected light enhanced. At all angles greater than the critical angle all light will be reflected. The depth of excitation can be controlled through the use of choice in excitation wavelength used to generate the evanescent wave [44].The critical angle can be calculated for two materials of different refractive index using the following formula derives from Snell's Law:

$$
\theta_{c}=\left(\frac{N_{2}}{N_{1}}\right)
$$

Where $\theta_{c}$ is the critical angle, $N_{1}$ is the angle of the first refractive index and $N_{2}$ is the second refractive index. In units of degrees

\subsubsection{Prism and objective based TIRF microscopy}

Objective and Prism type TIRF are two methods for exciting a sample bound to the surface of a slide or coverslip. Objective TIRF occurs through the excitation and collection of emission light by the objective. While with prism TIRF, the sample is excited from the top with the use of a prism and the emitted light is collected through an objective lenses positioned on the opposite side of the prism. Both techniques have their advantages and disadvantages: objective TIRF is easier but has slightly worse signalto-noise while prism TIRF is harder and use but produces better signal-to-noise.

The primary use of TIRF microscopy is to observe fluorescent signal attached to the surface of the slide. TIRF is typically used in experimental situations where high signal-to-noise ratios are required to obtain high quality data. A sensitive detector such as an electron multiplying charged coupled device electron 
multiplying charge coupled device (EMCCD) [41] or a scientific CMOS (sCMOS) are typically used because of their sensitivity [24]. TIRF is widely used in biological research fields like molecular biology, biochemistry and biophysics. One disadvantage is that TIRF like many other fluorescence techniques is limited in resolution to $\lambda / 2$ or half the wavelength of light. The application and development of new technologies for fluorescence observation has allowed researchers to develop super resolution imaging, which has been able to work beyond conventional limits of fluorescence microscopy.

\subsubsection{Methods of selective fluorescence observations}

Zero mode wave guides are a substrate that can be used for selective observation of fluorescence and use of the restriction of the excitation wavelength that is able to enter the structure and also provides a restricted observation volume. Zero mode wave guides are made of fused silica with a patterned aluminum overlay that has a set of specifically designed holes prepared by electron beam lithography followed by reactive ion etching [45]. By changing the specific diameter of the hole, the dimensions can be used to control the wavelength of light able to enter the zero mode wave guide device. Experiments performed with zero mode wave guides are done using prism TIRF. Volume restriction, down to a zeptolitre is possible and results in an increased signal-to-noise when examining conditions where there is a need for high concentrations of fluorescently labeled molecules in solution [46]. To the smFRET field that studies biomolecules, this means that experiments with fluorescent species at more physiological concentrations and at a greater range of concentrations are possible. Zero mode wave guide have been used to examine translation by the ribosome with both two and three color smFRET [47].

Gold and silver, noble metals, pattered metal nanostructures are a method for selective emission signal enhancement and can be tuned to enhance the wavelength of light by altering their structural dimensions and material makeup. Their ability to enhance the emission signal comes from their generation of localized electric field called surface plasmon resonance (SPR) [48]. An electrical field is generated when incoming photons interacts with the valence electron of the noble metal coated surface [49]. Noble metals like gold and silver are often used because of their tunable optical properties and relatively nonreactive nature when coming in contact with biological material [50]. Surface Plasmon Resonanceenhancing structures come in a variety of different shapes and sizes including nanoparticles [50], plasmonic bowtie nanoantennae [51], gold nanoshell [52], gold nanocages[53] as well as a variety other structures. 
Patterned noble metal nanostructured material represent an alternative to expensive conventional system used for the observation of fluorescence. Some systems have already been applied as a method for the detection of disease and may one day be the preferred method for diagnosis because of their advantages as being easily transportable and cost effective [45].

\subsubsection{Ribosome structure}

The Escherichia coli ribosome is a $\sim 2.5$ Mega Dalton (MDa) complex of RNA and protein, as compared to the eukaryotic ribosome which is roughly a 4.5 MDa complex. Ribosomes are made of two main subunits, which, when joined together, roughly form a spherical shape. The interior of the ribosomal subunits is primarily RNA with the proteins bound to the exterior, although there are a number of proteins that extend within the rRNA component of the ribosome. Escherichia coli ribosomes are made of two subunits, the small subunit which is made up of $1 \mathrm{rRNA}$, the $16 \mathrm{~S}$, and 21 proteins and a large subunit which is made of two rRNA, the $5 \mathrm{~S}$ and $23 \mathrm{~S}$, and $\sim 34$ proteins. An effort in recent history has been made to rename the ribosomal proteins for a more logical and consistent nomenclature. [54]. Our understanding of the structure of the ribosomes has been vastly improved by structural biology techniques, such as CryoEM and X-ray crystallography [55].

\subsubsection{X-ray crystallography and the ribosome}

Developed in the early 1920s, X-ray crystallography has been used to help study, develop and obtaining of structures of, alloys, minerals, small molecules, drugs, protein, nucleic acid and much more. X-ray crystallography but has been particularly important to the field of structural biology because of its use in the production of angstrom resolution structures of biomolecules [56].

The pursuit of high resolution ribosome structures had been ongoing for a number of years and starting in the 2000s labs were able to consistently obtain high resolutions structures several angstroms. This was recognized for what ended nearly three decades of work to crystalize and obtain a structures for the ribosome, some of the most influential players involved with ribosome X-ray crystallography were awarded the Nobel Prize in 2009, including: Ada Yonath, Venkatraman Ramakrishnan and Thomas Steitz.

The preparation of Archaea, Bacteria and Eukaryotic ribosomes in an assortment of state with various factors have been crystalized. Researchers have continued to focus on obtaining the highest resolution structures of the ribosome during various biologically relevant processes. Escherichia coli 
ribosomes have been crystalized with deacylated tRNA [57-59], multiple tRNA [60], acylated tRNA [61], in states like the pre translation state [62], the post translocation complex [60] and with a number of different factors like EF-Tu for example [63]. X-ray crystallographic studies continue to take on ambitiously and complex mechanistic questions of translation ever advancing techniques [64] with better resolution [65]. Many studies began with the study of Archaea and Bacteria and have since moved more toward Eukaryotic systems and the investigation human ribosome and diseases [66].

X-ray crystallography has helped to complement smFRET studies by providing information on dye placement sites and structures of the ribosome in various states during translation [67].

There are limits to X-ray crystallography and one of the primary disadvantages has been that the structure you obtain is the average of a crystal in a packed lattice which in often unlike its native conditions. The advancement of technologies used for cryo-electron microscopy have been able to overcome some of these obstacles.

\subsubsection{CryoEM and the ribosome}

In recent years cryoEM has become a powerful tool for the study of structural biology. Cryoelectron microscopy, a type of transmission electron microscopy (TEM), allows for the imaging of a sample which has been cryogenically frozen. Electron microscopy was first invented in around the 1930s; however, it was not until 1981, when the short article entitled "Vitrification of pure water" was published, that its real potential started to become realized [68]. Samples for cryoEM are flash frozen with the use of liquid ethane or propane to prepare the sample for visualization on the microscope [69]. Cryo-electron microscopy has grown to complement structural techniques like nuclear magnetic resonance and X-ray crystallography. In the past, cryoEM structures were often limited to a resolution of tens of angstroms, which because of recent technological advances has now become only a few angstroms.

The use of cryoEM to studying translation and the ribosome has provided a greater understanding of its mechanism [70]. Early studies produced numerous lower resolution images of the ribosome however the resolution restrictions did not completely prevent observation of the ribosome. In 1998, Joachim frank published a 15A resolution of the Escherichia coli ribosome in complex with fMET-tRNA-fMet with the use of cryoEM [71]. This was followed by the first observations of inter-subunit rotation by Joachim Frank, also using cryoEM [72]. 
One advantage and disadvantage of cryoEM over X-ray crystallography has been its ability to capture and short a multitude of different structures of the same molecule instead of just looking at the average. This has been used extensively when examining the ribosome because it is dynamic under equilibrium conditions. Numerous studies have examined the ribosome in preparation with specific factors, antibiotics and during specific processes [73]. Additional studies, have examined the ribosome throughout translation, by examining the ribosomes in known states of translocation and then subsequently analyzing the position of known dynamic moieties, like the L1 stalk, tRNA and subunit rotation, change [74].

Other papers have focused on obtaining high resolution structures $<3 \mathrm{~A}$ resolution such as the ribosome complex with the EF-Tu complex [63]. An extension of cryoEM called time-resolved cryoelectron microscopy has been shown to be a powerful technique in studying the ribosome. Time-resolved cryo-electron microscopy allows for the capture of images of a construct throughout a non-equilibrium process or set of state and has been applied to studying subunit association ribosomal inter-subunit rotation and translocation $[75,76]$.

With recent advances in the structure determination field by cryoEM and X-ray crystallography, a huge amount of information can obtained through snap shots of the ribosome. The study of real time dynamic processes of the ribosome are currently not accessible to cryoEM or X-ray crystallography and alternative techniques like smFRET can be used to report on the real time dynamics of a biomolecule.

\subsubsection{Ribosome function}

The ribosome is a complex macromolecular machine that functions to turn the information coded in a gene into protein. This is done through a multistep process which first begins with the transcription of DNA into RNA and then the translation of that RNA into protein by the ribosome. The regulation of protein production by the cell is critically important the survival and can take place through the numinous steps preceding translation or by the ribosomes itself. The many different component of translation that interacts with the ribosome have been shown to influence the mechanism of the ribosome [8]. Recently, the ribosome has been increasing thought of as a target of post translational modifications particularly including phosphorylation and ubiquitination [77]. The ribosome is made up of numerous interacting functional sites that facilitate the catalysis of protein synthesis. 


\subsubsection{Functional sites of the ribosome}

The ribosome supports multiple catalytic events throughout translation and disruption of a single process within the ribosome often results in a detrimental effect on protein synthesis. Notable sites critical to the ribosome include the tRNA binding sites, peptidyl transferase activity, mRNA entrance tunnel, decoding center and the L1 stalk.

The core of the ribosome is composed of three main binding sites, the amino acyl site (A-site), the peptidyl site (P-site) and exit site (E-site), that tRNA bind to during the elongation phase of translation. The A-site is responsible for the accommodated of amino acylated tRNA into the ribosome, with the use of EFTu-GTP. It is within the A-site that peptide bond formation occurs between the A-site tRNA and the P-site tRNA. Peptide bond formation is facilitated by the peptide transferase center, which helps the A-site tRNA remove the amino acids joined to P-site tRNA, to elongate the growing polypeptide. The peptidyl transferase center (PTC) is located on the 50S subunit and is composed of the $23 \mathrm{~S}$ rRNA component of the ribosome $[78,79]$. The P-site is occupied by a growing peptide chain after translocation of the ribosome and in preparation for the joining of additional aminoacyl tRNA. Removal of deacylated tRNA is facilitated by the exit site of the ribosome. (Figure 2)

During translation, the growing protein chain is threaded through the protein exit tunnel interactions between both the rRNA as well as proteins. The exit tunnel is approximately 80-100 angstroms long, around 10 angstroms at its narrowest and 20 angstroms at its widest point. It can fit approximately 20 amino acids in an extended confirmation. The four proteins, L22, L23, L24 and L29 which surround the protein portion of the exit tunnel, are thought to be interaction sites for various factors involved in export and folding [80].

The mRNA entrance tunnel facilitates the movement of the mRNA into the ribosome. The mRNA first enters the ribosome through three ribosomal proteins S3, S4 which are positioned on the head of the small subunit and S5 is bound to the body of the small subunit. The mRNA is then threaded up through an rRNA component of the ribosome into the decoding center. S3, S4 and S5 have been implicated in the unwinding of mRNA secondary structures such as hairpins or pseudoknots and are thought to possesses helicase activity which helps to facilitate the removal of downstream secondary structures that the ribosome might encounter and is thought to participate in ribosome frameshifting [81]. Ribosomal proteins S7, S11 
and S18 are thought to be involved in translation fidelity by restricting the mRNA within the exit channel [82].

The decoding center, located in the A and P sites is where the 16S organizes and controls the fidelity of the mRNA's codon and tRNA anticodon interactions [83]. Structurally the decoding center lies between the head and the body of the 30S subunit which is the curved path along which the mRNA lies.

The L1 stalk has been implicated in the ability of the ribosome to facilitate the removal of E-site tRNA from the ribosome post translocation as well as stabilization of the ribosome in the hybrid state [14, 84, 85]. L1 stalk actually contains two components, an rRNA component which is made of the 23S's helix 76, 77 and 78 and ribosomal protein L1 [86].

Together, the tRNA binding sites, peptidyl transferase activity, mRNA entrance tunnel, decoding center and the L1 stalk, allow the ribosome to synthesize proteins.

\subsubsection{Translation}

Protein synthesis is an essential process for cellular function. Proteins make up a large portion of the cells, structural, signaling and enzymatic function and the macromolecular complex responsible for synthesis of protein is the ribosome[87]. The process by which DNA is converted to RNA is called transcription and the process by which RNA is converted into protein is called translation.

The ribosome "reads" a single stranded mRNA three nucleotides at a time. This three nucleotide grouping, called a codon, is made up of combination of 4 possible nucleotides A U G and C. This differs from DNA because of the use of U instead of T. RNA's use of ribose sugar instead of DNA's deoxyribose makes RNA more labile because of the 2' OH's ability to react. With 4 possible nucleotides and 3 possible positions within a codon the number of unique combinations 64 . There are only 20 natural amino acids

which are encoded by those 64 combinations some of which are encoded multiple times. Additionally, there is one start codon AUG and 3 stop codons named amber (UAG), ochre (UAA), and opal/umber (UGA). The polymerization of individual amino acids linked to tRNA has been shown to be catalyzed by the PTC center of the ribosome. The polypeptide chain is joined together, from $\mathrm{N}$ to $\mathrm{C}$ terminus of each adjoining peptide, through a peptide bonds. The growing polypeptide exits the ribosome through a tunnel where it can then undergo a number of additional processes during or after synthesis like base modification, 
cleavage, co-translational folding and export which can result in the production of the desired peptide sequence.

Translation is a very specialized and complicated process which has been broken into three main phases, initiation, elongation and termination /recycling.

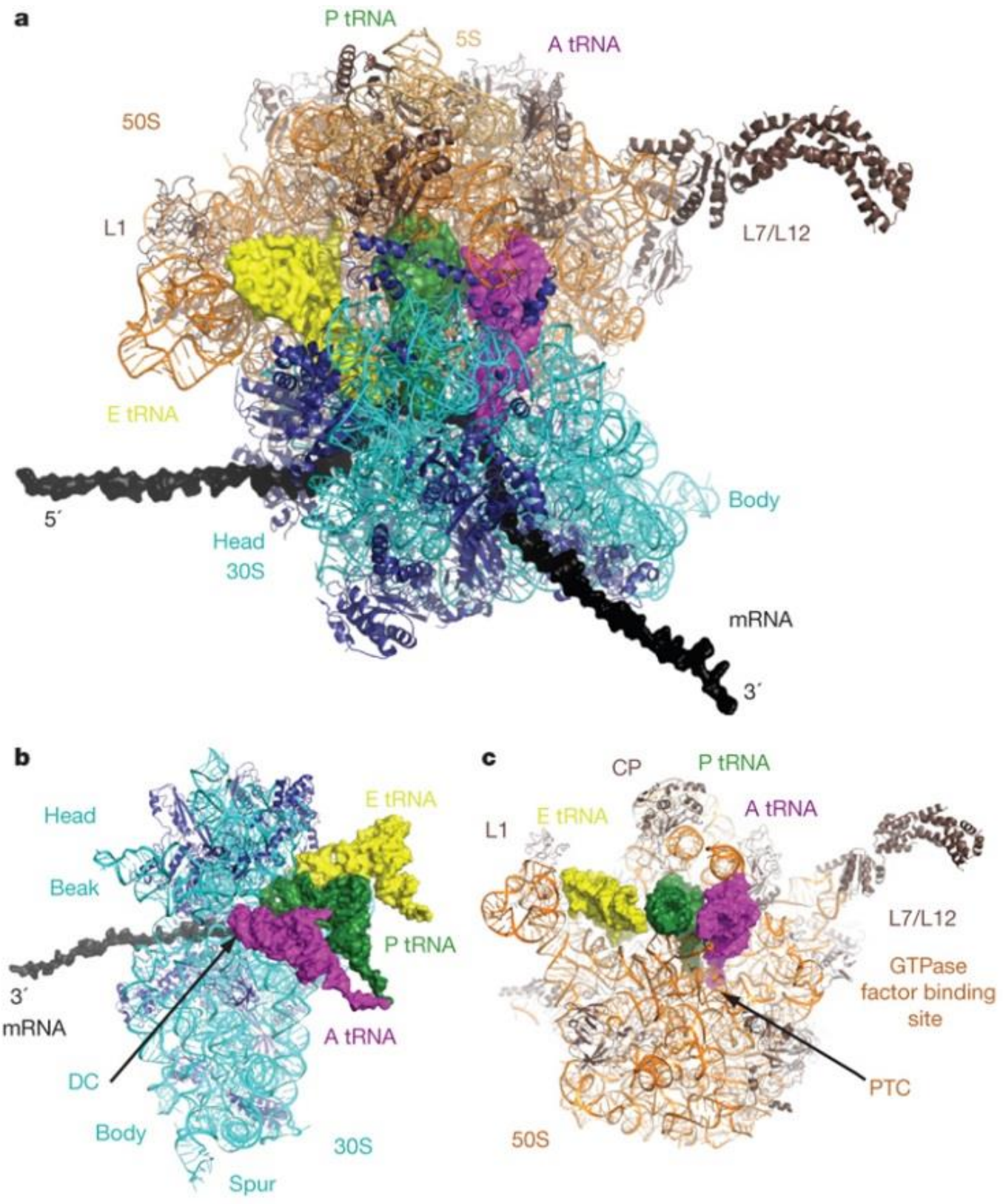

\subsubsection{Figure 2: 30S, $50 \mathrm{~S}$ and $70 \mathrm{~S}$ views of the ribosome}


a, 'Top' view of the 70S ribosome with mRNA and A- P- and E-site tRNAs. b, c, Exploded view of the 30S subunit (b) and 50S subunit (c). The structure of the L7/L12 arm [10] was fit onto the 70S ribosome [69], with mRNA elongated by modelling. This and all other figures were made with Pymol (Delano Scientific) and Photoshop (Adobe).

Reprint with permission from:

[88] Schmeing, T.M. and V. Ramakrishnan, What recent ribosome structures have revealed about the mechanism of translation. Nature, 2009. 461(7268): p. 1234-1242.

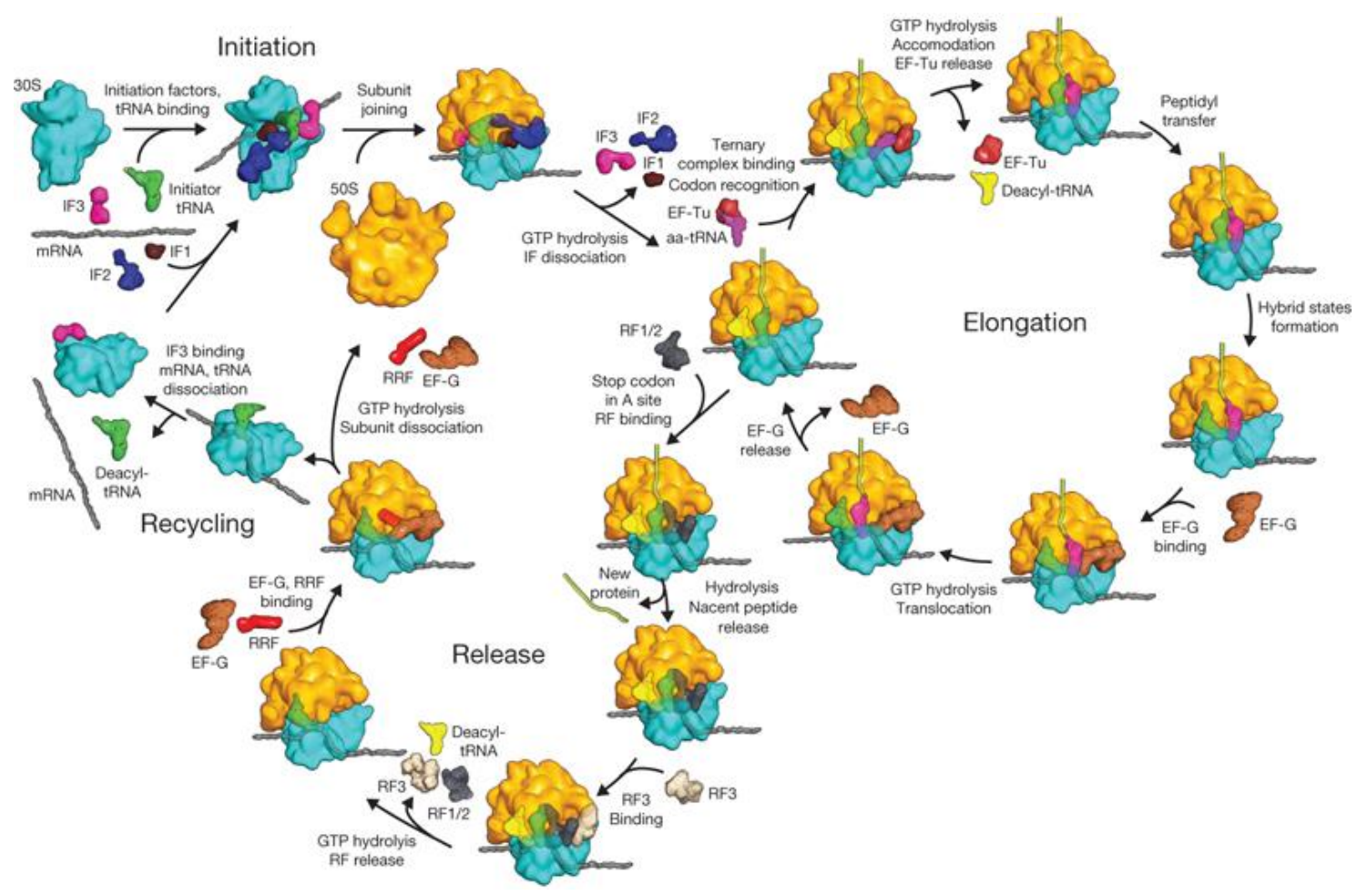

\subsubsection{Figure 3: Model of translation: Initiation, elongation, termination and recycling}

For simplicity, not all intermediate steps are shown. The colour scheme shown here is used consistently throughout this review. aa-tRNA, aminoacyl-tRNA; EF elongation factor; IF, initiation factor; RF, release factor.

Reprint with permission from:

[88] Schmeing, T.M. and V. Ramakrishnan, What recent ribosome structures have revealed about the mechanism of translation. Nature, 2009. 461(7268): p. 1234-1242. 


\subsubsection{Initiation}

Initiation is the process by which the ribosome is assembled mRNA in preparation for synthesis of a new protein. Initiation occurs in three main steps: The 30s ribosomal subunit binds two initiation factors (IF) IF-1 and IF-3 that prevent the 30S and 50S from joining prematurely. The mRNA then binds the 30S subunit at the Shine-Dalgarno sequence (SD). The start site is paired to the 16S's Anti-SD with the start codon positioned in the P-site, this is followed by the binding of IF-2 and acylated initiator tRNA Nformyl-Methionine-tRNA-N-formyl-Methionine binding. Followed by successful assembly of all three initiation factors, mRNA and initiator fMet-tRNA-fMet, hydrolysis of GTP occurs during a check step, and the $50 \mathrm{~S}$ is recruited. Afterwards, all of the initiation factors are released from the ribosome, leaving fMettRNA-fMet, tRNA, and the ribosome intact. (Figure 3)

\subsubsection{Elongation}

Translational Elongation is the successive addition of amino acids onto a growing peptide chain until the ribosome encounters a stop codon. Elongation factors and energy in the form of GTP are used to ensure fidelity and timely progression through translation. The ribosome proceeds through elongation by the successive accommodation of tRNA, with the help of Elongation factor Tu (EF-Tu). EF-Tu-GTP effectively acts as a proofreading step that checks the correct pairing of the codon anticodon interaction. Peptide bond formation between the amino terminus of the A-site tRNA and the carboxyl terminus of the P-site tRNA can then occur. Formation of the pre-translocated state of the ribosome has a deacylated tRNA in the P-site and a growing peptide chain attaching to the A-site tRNA. Following peptide bond formation, the ribosome must translocate to the next codon of the mRNA to translate protein. Elongation factor G (EFG) facilitates translocation through the hydrolysis of GTP, which results in the movement of the ribosome down the mRNA to the following codon. The process continues until the polypeptide is synthesized and stops when the ribosome encounters a stop codon [89]. (Figure 3)

\subsubsection{Termination}

Termination of protein synthesis occurs when the ribosome encounters one of the following stop codons: UAA UAG UGA. Termination begins with the positioning of the ribosome over a stop codon in the A-Site. RF-1 recognizes the termination codons UAG and UAA and RF-2 recognizes UGA and UAA. Once bound, these proteins promote the cleavage and release of the peptide from the P-Site tRNA. RF-3 
promotes release of RF-1 and RF-2 after polypeptides release [90]. There are a number of interesting control mechanism involved with release including the observation that RF-3 increases the affinity of RF-1 and RF-2 for the ribosome [91].

Ribosome recycling is the process by which the cell is able to reuse the ribosome to produce additional proteins. Ribosome recycling factor (RRF) together with EF-G and the hydrolysis of GTP is required for the dissociation of the 50S subunit after termination [90]. This EF-G-GTP hydrolysis leads to dissociation of the 50S and 30S tRNA-mRNA complex EF-G and RRF are replaced by IF-3 which promotes dissociation of the tRNA. (Figure 3)

\subsubsection{Using smFRET to examine large scale dynamic motions of the ribosome}

SmFRET reports on real time dynamic information and has been used extensively to observe the dynamics and binding of macromolecules in order to investigate their mechanism. FRET is the process by which energy can be non-radiatively transferred from a donor fluorophore to an acceptor fluorophore over a defined distance and has been adapted for single molecules with the use of a prism TIRF microscope. SmFRET was developed by TJ Ha in Shimon Weiss's lab. Their seminal manuscript was published in 1996 [92] and is based on the properties which were first described in 1946 by Theodor Förster in the paper, "energy migration and fluorescence" [93]. Since its inceptions, smFRET has been used to study numerous processes involving most important biological processes including ribosome dynamics [11, 17, 94, 95].

\subsubsection{Subunit rotation}

Subunit rotation is the counter clockwise rotation of the $30 \mathrm{~S}$ in relation to $50 \mathrm{~S}$ when viewed from the top of the 70S. Subunit rotation was first proposed to exist by Joachim Frank with its observation by cryoEM [72]. Bulk FRET was later used to investigate the coupling of tRNA hybrid state formation and rotation of the ribosomal subunit after translocation [9]. It was found that, by linking the subunits together, they lost their activity, further supporting the hypothesis that subunit rotation is critical for ribosomal function [96]. SmFRET experiments found that the ribosome was a Brownian ratchet and that at room temperature the ribosome is able to transition between its two rotation states, the rotated and non-rotated state [8]. Subunit rotation was reported on by the Puglisi lab however their construct used rRNA extension in order to label their construct and their results indicated that the ribosome would only transition when supplied with energy. These results are in conflict with the observation that the ribosome is able to 
spontaneously fluctuate between the rotated and non-rotated state. The addition of the rRNA extensions has been hypothesized to be the reason why the construct used was unable to freely transition between states [97]. Experimentation by other groups has shown that antibiotics that bind to the ribosome are able to shift its equilibrium of states [98]. It has also been shown that point mutations made between important interacting ribosomal proteins of the ribosome can disrupt the ribosome's native equilibrium of states [99]. In recent years, new constructs have been developed to examine inter-subunit rotation and continue to support the hypothesis of the ribosome as a Brownian ratchet [100].

\subsubsection{L1 stalk motion}

L1 stalk motion is the movement of the rRNA and protein extensions located by the E-site of the ribosome that is responsible for the removal of E site tRNA and helps to stabilize the ribosome in the hybrid state. Numerous groups have used a variety of different smFRET constructs to examine the L1 stalk with tRNA and other factors bound to the ribosome. Two different groups using two different labeling schemes were able to report on the dynamics of the L1 stalk. Both groups reported similar findings, however Fei et al. found that the L1 stalk could occupy two states [13] while Cornish et al. were able to observe three states, the open, the closed and the half closed state, the latter of which is only populated when the solution conditions are supplemented with excess tRNA [14]. It was also found that the L1 stalk interacts with the tRNA within the ribosome during elongation [84]. The L1 stalk has also been shown to be important to the global dynamic and structural rearrangement of the ribosome required for productive translocation [101]. The use of two and three color smFRET to examine the dynamics of the L1 stalk and tRNA motion uncovered that both motions are loosely coupled, independent processes that must all come together for the ribosome to efficiently translocate [102] [99].

\subsection{3 tRNA motion}

tRNA motion is the movement of the tRNA through the A, P and E-site of the ribosome as it progresses through translation. smFRET helped to determine that, not only can tRNA in the ribosome occupy the A, P and E site of the ribosome, but they can also form hybrid states which include the A/P and $\mathrm{P} / \mathrm{E}$ states of the ribosome [103] [10] [12] [11]. There have been numerous studies using constructs that examine the dynamics of the L1 stalk to study other mechanistic details of the ribosome such as how tRNA motion is coordinated with the other dynamic motions of the ribosome [101] [84] [99] [104]. 


\subsubsection{References}

1. Narla, A. and B.L. Ebert, Ribosomopathies: human disorders of ribosome dysfunction. Blood, 2010. 115(16): p. 3196-3205.

2. Palade, G.E., A SMALL PARTICULATE COMPONENT OF THE CYTOPLASM. The Journal of Biophysical and Biochemical Cytology, 1955. 1(1): p. 59-68.

3. Wittmann, H.G., et al., Crystallization of Escherichia coli ribosomes. FEBS Letters, 1982. 146(1): p. $217-220$.

4. Vainshtein, B.K. and S.D. Trakhanov, Crystallization of Ribosomes, Ribosomal Subunits, and Individual Ribosomal Proteins, in Growth of Crystals: Volume 16, K.S. Bagdasarov and É.L. Lube, Editors. 1991, Springer US: Boston, MA. p. 169-180.

5. Yonath, A., J. Müssig, and H.G. Wittmann, Parameters for crystal growth of ribosomal subunits. Journal of Cellular Biochemistry, 1982. 19(2): p. 145-155.

6. Wimberly, B.T., et al., Structure of the 30 S ribosomal subunit. Nature, 2000. 407(6802): p. 327339.

7. Ban, N., et al., The Complete Atomic Structure of the Large Ribosomal Subunit at $2.4 \AA$ Resolution. Science, 2000. 289(5481): p. 905-920.

8. Cornish, P.V., et al., Spontaneous Intersubunit Rotation in Single Ribosomes. Molecular Cell, 2008. 30(5): p. 578-588.

9. Ermolenko, D.N., et al., Observation of Intersubunit Movement of the Ribosome in Solution Using FRET. Journal of Molecular Biology, 2007. 370(3): p. 530-540.

10. Blanchard, S.C., et al., tRNA dynamics on the ribosome during translation. Proceedings of the National Academy of Sciences of the United States of America, 2004. 101(35): p. 12893-12898.

11. Munro, J.B., et al., Single Molecule Series A New View of Protein Synthesis: Mapping the Free Energy Landscape of the Ribosome Using Single-Molecule FRET. Biopolymers, 2008. 89(7): p. $565-577$.

12. Munro, J.B., et al., Identification of Two Distinct Hybrid State Intermediates On the Ribosome. Molecular cell, 2007. 25(4): p. 505-517. 
13. Fei, J., et al., Allosteric collaboration between elongation factor G and the ribosomal L1 stalk directs tRNA movements during translation. Proceedings of the National Academy of Sciences of the United States of America, 2009. 106(37): p. 15702-15707.

14. Cornish, P.V., et al., Following movement of the L1 stalk between three functional states in single ribosomes. Proceedings of the National Academy of Sciences, 2009. 106(8): p. 2571-2576.

15. Caban, K. and R.L. Gonzalez, The emerging role of rectified thermal fluctuations in initiator aatRNA- and start codon selection during translation initiation. Biochimie, 2015. 114: p. 30-38.

16. Salsi, E., et al., Movement of Elongation Factor G between Compact and Extended Conformations. Journal of molecular biology, 2015. 427(2): p. 454-467.

17. Sternberg, S.H., et al., Translation factors direct intrinsic ribosome dynamics during translation termination and ribosome recycling. Nat Struct Mol Biol, 2009. 16(8): p. 861-868.

18. Guo, Z. and H.F. Noller, Rotation of the head of the $30 S$ ribosomal subunit during mRNA translocation. Proceedings of the National Academy of Sciences, 2012. 109(50): p. 20391-20394.

19. Kim, H.-K., et al., A frameshifting stimulatory stem loop destabilizes the hybrid state and impedes ribosomal translocation. Proceedings of the National Academy of Sciences of the United States of America, 2014. 111(15): p. 5538-5543.

20. Kim, H.-K. and J.I. Tinoco, EF-G catalyzed translocation dynamics in the presence of ribosomal frameshifting stimulatory signals. Nucleic Acids Research, 2017. 45(5): p. 2865-2874.

21. Chen, B., et al., EttA regulates translation by binding to the ribosomal E site and restricting ribosome-tRNA dynamics. Nature structural \& molecular biology, 2014. 21(2): p. 152-159.

22. Lakowicz, J.R., Principles of fluorescence spectroscopy. 2006, New York: Springer.

23. Doornkaat-Koolman, J., Friesische Ortsnamen.

24. Roy, R., S. Hohng, and T. Ha, A Practical Guide to Single Molecule FRET. Nat Methods, 2008. 5(6): p. 507-16.

25. Pisoni, D.S., et al., Symmetrical and Asymmetrical Cyanine Dyes. Synthesis, Spectral Properties, and BSA Association Study. The Journal of Organic Chemistry, 2014. 79(12): p. 5511-5520.

26. Altman, R.B., et al., cyanine fluorophore derivatives with enhanced photostability. Nature methods, 2011.9(1): p. 68-71. 
27. van der Meer, B.W., Kappa-squared: from nuisance to new sense. Reviews in Molecular Biotechnology, 2002. 82(3): p. 181-196.

28. Hohng, S., C. Joo, and T. Ha, Single-Molecule Three-Color FRET. Biophysical Journal, 2004. 87(2): p. 1328-1337.

29. Lee, S., J. Lee, and S. Hohng, Single-Molecule Three-Color FRET with Both Negligible Spectral Overlap and Long Observation Time. PLoS ONE, 2010. 5(8): p. e12270.

30. EXPANDING THE GENETIC CODE. Annual Review of Biophysics and Biomolecular Structure, 2006. 35(1): p. 225-249.

31. Noren, C., et al., A general method for site-specific incorporation of unnatural amino acids into proteins. Science, 1989. 244(4901): p. 182-188.

32. Brustad, E.M., et al., A General and Efficient Method for the Site-Specific Dual-Labeling of Proteins for Single Molecule Fluorescence Resonance Energy Transfer. Journal of the American Chemical Society, 2008. 130(52): p. 17664-17665.

33. Liu, W., et al., Genetic incorporation of unnatural amino acids into proteins in mammalian cells. Nat Meth, 2007. 4(3): p. 239-244.

34. Wang, F., et al., Genetic Incorporation of Unnatural Amino Acids into Proteins in Mycobacterium tuberculosis. PLOS ONE, 2010. 5(2): p. e9354.

35. Keppler, A., et al., A general method for the covalent labeling of fusion proteins with small molecules in vivo. Nat Biotech, 2003. 21(1): p. 86-89.

36. Gautier, A., et al., An Engineered Protein Tag for Multiprotein Labeling in Living Cells. Chemistry \& Biology, 2008. 15(2): p. 128-136.

37. Hwang, H. and S. Myong, Protein induced fluorescence enhancement (PIFE) for probing proteinnucleic acid interactions. Chemical Society reviews, 2014. 43(4): p. 1221-1229.

38. Berney, C. and G. Danuser, FRET or No FRET: A Quantitative Comparison. Biophys J, 2003. 84(6): p. 3992-4010.

39. McCann, J.J., et al., Optimizing Methods to Recover Absolute FRET Efficiency from Immobilized Single Molecules. Biophys J, 2010. 99(3): p. 961-70. 
40. Hohng, S., C. Joo, and T. Ha, Single-Molecule Three-Color FRET. Biophys J, 2004. 87(2): p. 1328-37.

41. Combs, C.A., Fluorescence Microscopy: A Concise Guide to Current Imaging Methods. Current protocols in neuroscience / editorial board, Jacqueline N. Crawley ... [et al.], 2010. 0 2: p. Unit2.1Unit2.1.

42. Ha, T., et al., Probing the interaction between two single molecules: fluorescence resonance energy transfer between a single donor and a single acceptor. Proceedings of the National Academy of Sciences, 1996. 93(13): p. 6264-6268.

43. Roy, R., S. Hohng, and T. Ha, A Practical Guide to Single Molecule FRET. Nature methods, 2008. 5(6): p. 507-516.

44. Fish, K.N., Total Internal Reflection Fluorescence (TIRF) Microscopy. Current protocols in cytometry / editorial board, J. Paul Robinson, managing editor ... [et al.], 2009. 0 12: p. Unit12.18Unit12.18.

45. Levene, M.J., et al., Zero-Mode Waveguides for Single-Molecule Analysis at High Concentrations. Science, 2003. 299(5607): p. 682-686.

46. Samiee, K.T., et al., Zero Mode Waveguides for Single-Molecule Spectroscopy on Lipid Membranes. Biophysical Journal, 2006. 90(9): p. 3288-3299.

47. Chen, J., et al., High-throughput platform for real-time monitoring of biological processes by multicolor single-molecule fluorescence. Proceedings of the National Academy of Sciences, 2014. 111(2): p. 664-669.

48. Bronson, J.E., et al., Learning Rates and States from Biophysical Time Series: A Bayesian Approach to Model Selection and Single-Molecule FRET Data. Biophysical Journal, 2009. 97(12): p. 3196-3205.

49. Aslan, K., J.R. Lakowicz, and C.D. Geddes, Plasmon light scattering in biology and medicine: new sensing approaches, visions and perspectives. Current Opinion in Chemical Biology, 2005. 9(5): p. 538-544. 
50. Jain, P.K., et al., Review of Some Interesting Surface Plasmon Resonance-enhanced Properties of Noble Metal Nanoparticles and Their Applications to Biosystems. Plasmonics, 2007. 2(3): p. 107118.

51. Dodson, S., et al., Optimizing Electromagnetic Hotspots in Plasmonic Bowtie Nanoantennae. The Journal of Physical Chemistry Letters, 2013. 4(3): p. 496-501.

52. Loo, C., et al., Nanoshell-Enabled Photonics-Based Imaging and Therapy of Cancer. Technology in Cancer Research \& Treatment, 2004. 3(1): p. 33-40.

53. Chen, J., et al., Immuno Gold Nanocages with Tailored Optical Properties for Targeted Photothermal Destruction of Cancer Cells. Nano Letters, 2007. 7(5): p. 1318-1322.

54. Ban, N., et al., A new system for naming ribosomal proteins. Current opinion in structural biology, 2014. 24: p. 165-169.

55. Campbell, I.D., The march of structural biology. Nat Rev Mol Cell Biol, 2002. 3(5): p. 377-381.

56. Ling, C. and D.N. Ermolenko, Structural insights into ribosome translocation. Wiley Interdisciplinary Reviews: RNA, 2016. 7(5): p. 620-636.

57. Selmer, M., et al., Structure of the $70<e m>S</ e m>$ Ribosome Complexed with mRNA and tRNA. Science, 2006. 313(5795): p. 1935-1942.

58. Feng, S., Y. Chen, and Y.-G. Gao, Crystal Structure of 70S Ribosome with Both Cognate tRNAs in the E and P Sites Representing an Authentic Elongation Complex. PLOS ONE, 2013. 8(3): p. e58829.

59. Cate, J.H., et al., X-ray Crystal Structures of $70<e m>S</ e m>$ Ribosome Functional Complexes. Science, 1999. 285(5436): p. 2095-2104.

60. Korostelev, A., et al., Crystal Structure of a 70S Ribosome-tRNA Complex Reveals Functional Interactions and Rearrangements. Cell, 2006. 126(6): p. 1065-1077.

61. Rozov, A., et al., Structural insights into the translational infidelity mechanism. Nature Communications, 2015. 6: p. 7251.

62. Li, W., et al., Activation of GTP hydrolysis in mRNA-tRNA translocation by elongation factor G. Science Advances, 2015. 1(4). 
63. Fischer, N., et al., Structure of the E. coli ribosome-EF-Tu complex at $<3$ A resolution by Cscorrected cryo-EM. Nature, 2015. 520(7548): p. 567-570.

64. Cheng, Y., Single-particle cryo-EM at crystallographic resolution. Cell, 2015. 161(3): p. $450-457$.

65. Shi, Y., A Glimpse of Structural Biology through X-Ray Crystallography. Cell, 2014. 159(5): p. 995-1014.

66. Budkevich, T., et al., Structure and dynamics of the mammalian ribosomal pre-translocation complex. Molecular cell, 2011. 44(2): p. 214-224.

67. Dunkle, J.A., et al., Structures of the bacterial ribosome in classical and hybrid states of tRNA binding. Science (New York, N.Y.), 2011. 332(6032): p. 981-984.

68. Mayer, E., Vitrification of pure liquid water. Journal of Microscopy, 1985. 140(1): p. 3-15.

69. Thompson, R.F., et al., An introduction to sample preparation and imaging by cryo-electron microscopy for structural biology. Methods, 2016. 100: p. 3-15.

70. Subramaniam, S., W. Kuhlbrandt, and R. Henderson, CryoEM at IUCrJ: a new era. IUCrJ, 2016. 3(1): p. 3-7.

71. Malhotra, A., et al., Escherichia coli $70 \mathrm{~S}$ ribosome at $15 \AA$ A resolution by cryo-electron microscopy: localization of fmet-tRNAfMet and fitting of L1 protein1. Journal of Molecular Biology, 1998. 280(1): p. 103-116.

72. Frank, J. and R.K. Agrawal, A ratchet-like inter-subunit reorganization of the ribosome during translocation. Nature, 2000. 406(6793): p. 318-322.

73. Zhou, J., et al., Crystal Structures of EF-G-Ribosome Complexes Trapped in Intermediate States of Translocation. Science (New York, N.Y.), 2013. 340(6140): p. 1236086-1236086.

74. Bock, L.V., et al., Energy barriers and driving forces in tRNA translocation through the ribosome. Nat Struct Mol Biol, 2013. 20(12): p. 1390-1396.

75. Chen, B., et al., Structural Dynamics of Ribosome Subunit Association Studied by MixingSpraying Time-Resolved Cryogenic Electron Microscopy. Structure, 2015. 23(6): p. 1097-1105.

76. Fischer, N., et al., Ribosome dynamics and tRNA movement by time-resolved electron cryomicroscopy. Nature, 2010. 466(7304): p. 329-333. 
77. Simsek, D. and M. Barna, An emerging role for the ribosome as a nexus for post-translational modifications. Current Opinion in Cell Biology, 2017. 45: p. 92-101.

78. Sievers, A., et al., The ribosome as an entropy trap. Proceedings of the National Academy of Sciences of the United States of America, 2004. 101(21): p. 7897-7901.

79. Ś Swiderek, K., et al., Peptide Bond Formation Mechanism Catalyzed by Ribosome. Journal of the American Chemical Society, 2015. 137(37): p. 12024-12034.

80. Kramer, G., et al., The ribosome as a platform for co-translational processing, folding and targeting of newly synthesized proteins. Nat Struct Mol Biol, 2009. 16(6): p. 589-597.

81. Takyar, S., R.P. Hickerson, and H.F. Noller, mRNA Helicase Activity of the Ribosome. Cell, 2005. 120(1): p. 49-58.

82. Kurkcuoglu, O., et al., The ribosome structure controls and directs mRNA entry, translocation and exit dynamics. Physical biology, 2008. 5(4): p. 046005-046005.

83. Schluenzen, F., et al., Structure of Functionally Activated Small Ribosomal Subunit at $3.3 \AA$ Resolution. Cell, 2000. 102(5): p. 615-623.

84. Fei, J., et al., Coupling of Ribosomal L1 Stalk and tRNA Dynamics during Translation Elongation. Molecular Cell, 2008. 30(3): p. 348-359.

85. Trabuco, L.G., et al., The role of L1 stalk:tRNA interaction in the ribosome elongation cycle. Journal of molecular biology, 2010. 402(4): p. 741-760.

86. Réblová, K., J. Šponer, and F. Lankaš, Structure and mechanical properties of the ribosomal L1 stalk three-way junction. Nucleic Acids Research, 2012. 40(13): p. 6290-6303.

87. Moore, P.B., How Should We Think About the Ribosome? Annual Review of Biophysics, 2012. 41(1): p. 1-19.

88. Schmeing, T.M. and V. Ramakrishnan, What recent ribosome structures have revealed about the mechanism of translation. Nature, 2009. 461(7268): p. 1234-1242.

89. Evans, R.N., et al., The structure of LepA, the ribosomal back translocase. Proceedings of the National Academy of Sciences of the United States of America, 2008. 105(12): p. 4673-4678.

90. Karimi, R., et al., Novel Roles for Classical Factors at the Interface between Translation Termination and Initiation. Molecular Cell. 3(5): p. 601-609. 
91. Klaholz, B.P., et al., Structure of the Escherichia coli ribosomal termination complex with release factor 2. Nature, 2003. 421(6918): p. 90-94.

92. Ha, T., et al., Probing the interaction between two single molecules: fluorescence resonance energy transfer between a single donor and a single acceptor. Proceedings of the National Academy of Sciences of the United States of America, 1996. 93(13): p. 6264-6268.

93. Förster, T., Energy migration and fluorescence. Journal of Biomedical Optics, 2012. 17(1): p. 0110021-01100210.

94. Wang, J., K. Caban, and R.L. Gonzalez, Ribosomal initiation complex-driven changes in the stability and dynamics of initiation factor 2 regulate the fidelity of translation initiation. Journal of molecular biology, 2015. 427(9): p. 1819-1834.

95. Tinoco, I. and R.L. Gonzalez, Biological mechanisms, one molecule at a time. Genes \& Development, 2011. 25(12): p. 1205-1231.

96. Horan, L.H. and H.F. Noller, Intersubunit movement is required for ribosomal translocation. Proceedings of the National Academy of Sciences of the United States of America, 2007. 104(12): p. 4881-4885.

97. Marshall, R.A., M. Dorywalska, and J.D. Puglisi, Irreversible chemical steps control intersubunit dynamics during translation. Proceedings of the National Academy of Sciences, 2008. 105(40): p. 15364-15369.

98. Ermolenko, D.N., et al., Antibiotics that bind to the A site of the large ribosomal subunit can induce mRNA translocation. RNA, 2013. 19(2): p. 158-166.

99. Ning, W., J. Fei, and R.L. Gonzalez, The ribosome uses cooperative conformational changes to maximize and regulate the efficiency of translation. Proceedings of the National Academy of Sciences of the United States of America, 2014. 111(33): p. 12073-12078.

100. Wasserman, M.R., et al., Multiperspective smFRET reveals rate-determining late intermediates of ribosomal translocation. Nat Struct Mol Biol, 2016. 23(4): p. 333-341.

101. Fei, J., et al., Transfer RNA-mediated regulation of ribosome dynamics during protein synthesis. Nat Struct Mol Biol, 2011. 18(9): p. 1043-1051. 
102. Munro, J.B., et al., A fast dynamic mode of the EF-G-bound ribosome. The EMBO Journal, 2010. 29(4): p. 770-781.

103. Blanchard, S.C., et al., tRNA selection and kinetic proofreading in translation. Nat Struct Mol Biol, 2004. 11(10): p. 1008-1014.

104. Frank, J. and R.L. Gonzalez, Structure and Dynamics of a Processive Brownian Motor: The Translating Ribosome. Annual review of biochemistry, 2010. 79: p. 381-412. 


\subsubsection{Chapter 2: Fluorescence enhancement from nano-gap embedded plasmonic gratings by a novel fabrication technique with HD-DVD}

As seen in: Bhatnagar, K., Pathak, A., Menke, D., Cornish, P. V., Gangopadhyay, K., Korampally, V., \& Gangopadhyay, S. (2012). Fluorescence enhancement from nano-gap embedded plasmonic gratings by a novel fabrication technique with HD-DVD. Nanotechnology, 23(49), [495201]. DOI: 10.1088/0957$4484 / 23 / 49 / 495201$

\subsubsection{Abstract}

We demonstrate strong electromagnetic field enhancement from nano-gaps embedded in silver gratings for visible wavelengths. These structures fabricated using a store-bought HD-DVD worth \$10 and conventional micro-contact printing techniques have shown maximum fluorescence enhancement factors of up to 118 times when compared to a glass substrate under epi-fluorescent conditions. The novel fabrication procedure provides for the development of a cost-effective and facile plasmonic substrate for low-level chemical and biological detection. Electromagnetic field simulations were also performed that reveal the strong field confinement in the nano-gap region embedded in the silver grating, which is attributed to the combined effect of localized as well as propagating surface plasmons.

\subsubsection{Introduction}

Surface plasmons have been used extensively in the past few decades for applications in subwavelength optics, data storage, optoelectronic circuits, diffraction-limited microscopy and bio-photonics. Recent years have seen a significant growth in the development of plasmonic nanostructures because of their ability to concentrate electromagnetic energy at sub-wavelength scales $[1,2]$. The phenomenon of surface plasmon resonance (SPR) can enhance the interaction between the incident light and the metallic nanostructures, leading to highly localized electric fields within a few hundred nanometers [3, 4]. By confining light to volumes as small as $1 \mathrm{~nm} 3$, researchers have made optical wave guiding and imaging possible beyond the diffraction limit $[5,6]$. Recent studies of novel metal nanostructures have unraveled a plethora of applications that utilize the near-field localization of light for signal enhancement $[7,8]$ as well 
as signal propagation [9]. Fluorescence spectroscopy, on the other hand, has emerged as one of the key techniques for performing basic research to clinical diagnostics and plays a key role in ultrasensitive chemical and biological threat sensors [10-12]. However, there has been a growing need to improve the contrast levels in fluorescence images and the limit of detection for fluorescence based sensing. The advent of plasmonics in recent years has provided some of the answers required to improve the quality of fluorescence microscopy and spectroscopy through surface patterning and novel geometries [13, 14]. The enhanced electromagnetic field produced by plasmonic nanostructures can be used to couple light to fluorescent dye molecules in the immediate environment, thereby providing the extreme signal amplification necessary for detecting trace quantities of biomolecules tagged with fluorophores [15]. Furthermore, the presence of nearby metallic films or nanoparticles has been known to profoundly affect the emission characteristics of fluorophores in terms of increasing the quantum yields of weakly fluorescent molecules, reduced lifetimes and subsequently improved photostabilities [16].

Propagating surface plasmons are charge oscillations present on a planar metal-dielectric interface with propagation lengths on the order of hundreds of microns [17-19]. A proficient way to couple propagating surface plasmons to incident light is through the use of metal gratings [20, 21]. These periodic structures provide additional optical momentum to the incoming incident light, necessary for exciting the surface plasmons [19]. The surface plasmon resonance angle $\theta$ SPR, can be easily calculated theoretically from momentum matching conditions including the dispersion relationship in equation (1).

$$
\begin{aligned}
k_{\mathrm{SP}}= & \frac{\omega}{c} \sqrt{\frac{\varepsilon_{\mathrm{m}} \varepsilon_{\mathrm{d}}}{\varepsilon_{\mathrm{m}}+\varepsilon_{\mathrm{d}}}}=\frac{\omega}{c} \sqrt{\varepsilon_{\mathrm{d}}} \sin \theta_{\mathrm{SPR}} \pm m \frac{2 \pi}{\Lambda} \\
& (m= \pm 1,2,3, \ldots)
\end{aligned}
$$

where $\omega$ is the angular frequency of the incident light, $\mathrm{c}$ is the speed of light in vacuum, $\mathrm{\varepsilon m}$ is the dielectric constant for the metal, $\varepsilon d$ is the dielectric constant for the medium surrounding the metal, $\Lambda$ is the grating pitch, $\mathrm{kSP}$ is the wavevector of the surface plasmon, and $\mathrm{m}$ is an integer. Equation (1) can be used to accurately predict the resonance angle for a periodic pattern in one-dimension. Both 1D and 2D metal grating structures have been fabricated and shown to provide considerable signal enhancements using epifluorescence microscopy $[20,22]$.

Metal nanoparticles and metallic nano-gaps on the other hand exhibit surface plasmons that are non-propagating in nature and are known as localized surface plasmons [23, 24]. Coupling an incident 
photon to such a nanostructure can be achieved by selecting the resonant wavelength, which depends on the shape, size, and dielectric environment of the metallic nano-gap. Metallic nano-gap bow-ties as well as bow-tie arrays have been fabricated using e-beam lithography and used to observe individual fluorescent molecules attached to the surface owing to extreme electromagnetic field enhancement within the bow-tie nano-gap region using confocal microscopy [16].

One can now envision a nanostructure that utilizes the combined effect of both the periodic metal gratings and the metallic nano-gap. In this case, the propagating surface plasmon polaritons (SPPs) travel as surface waves with high field intensity towards the metallic nano-gap, where the sudden field discontinuity causes 'extreme crowding' of the surface charges, leading to very high field intensities [25-30]. The field enhancement within the gap can be expected to be on the order of $|\varepsilon \mathrm{m} / \varepsilon \mathrm{d}|$ owing to electromagnetic boundary conditions at the interface [31]. Hence, an individual nano-gap on a flat film will show a field enhancement of $|\varepsilon \mathrm{m} / \varepsilon \mathrm{d}|$, but a nano-gap embedded in a grating surface should show $|\varepsilon \mathrm{m} / \varepsilon \mathrm{d}|$ times the electromagnetic enhancement due to the coupling of surface plasmon polaritons. However, it is also important to remember that this enhancement is dependent on the size and dimensions of the nano-gap itself. It has been shown experimentally and computationally that the field enhancement increases as the nano-gap width decreases $[32,33]$. Since these structures combine both the localized surface plasmons (LPs) from the metallic nano-gaps as well as the SPPs from the metal gratings, the extreme field intensities can be used to excite fluorescent molecules in the vicinity, enabling the detection of very low concentrations of antibodies and proteins tagged with fluorophores, down to the single molecule level. Genevet et al exploited this idea and fabricated periodic arrays of nano-gaps and achieved up to 1000-fold field enhancements using a four-wave mixing setup [27].

Recent studies have shown the fabrication of sub-wavelength patterned metallic nanostructures using two-beam interference lithography, electron beam lithography and nano-imprint lithography [34] for enhanced fluorescence detection, microscopic imaging and surface enhance Raman spectroscopy (SERS) $[16,35,36]$. Although these techniques provide highly precise periodic structures and geometries on the nanoscale, they involve high cost and complexity in fabrication. Commercially available optical media on the other hand have drawn the attention of many researchers in the past few years as these offer an inexpensive alternative to fabrication of gratings $[37,38]$. CDs, DVDs and other optical disks have periodic 
gratings in the form of tracks on the polycarbonate substrate that can be coated with metal films to form plasmonic metal gratings [39-41]. A disadvantage with this method, however, is that these polycarbonate surfaces have to be tuned to obtain sharp resonance peaks by chemical etching methods, which have issues with reliability and reproducibility.

In this work, we present a novel fabrication technique for producing polymethylsilsesquioxane (PMSSQ) based gratings embedded with nano-gaps using micro-contact printing [42, 43], where the starting master pattern is obtained from commercially available high-definition (HD) DVD-Rs. The nanogaps occur spontaneously within the grating structure as a result of tensile strain in the elastomeric PDMS stamp during the printing process [44-46] (see supporting information for details available at stacks.iop.org/Nano/23/495201/mmedia). This method eliminates the high cost of production involved in conventional lithography and reactive ion-etching (RIE) procedures, providing substrates that are relatively fast, inexpensive and easy to fabricate. These nano-gap embedded PMSSQ gratings can be used as a base pattern for depositing metal layers for plasmonic nanostructures. We have produced nano-gap embedded silver gratings using this method for surface plasmon enhanced fluorescence and single molecule detection. Precise control of the nanofabrication of these structures can open doors to applications in diffractionlimited optics, optoelectronic circuits, and the observation of single molecule dynamics.

\subsubsection{Materials and methods}

For the fabrication process, polydimethylsiloxane (PDMS) $\mu$-contact printing was used to stamp the nano-gap embedded gratings on glass substrates using a dilute PMSSQ based ink. A commercially available HD DVD-R was dissected into two parts to reveal the grating pattern on the inner side of the polycarbonate substrate. The substrate was cleaned using isopropanol to dissolve the dye coating layer covering the periodic pattern on the HD DVDs. The pattern was replicated using PDMS elastomer with a ratio of 5:1 for the PDMS base to the cross-linking agent (Sylgard® 184 silicone elastomer kit available through Dow Corning, Inc.). The grating pattern now being transferred to the PDMS was used as a stamp to print the pattern onto a glass substrate. The unique fabrication procedure utilizes a $3 \%$ by weight solution of PMSSQ (Techneglas Technical Products, GR-650F) in ethanol (Sigma Aldrich, 99\%) as the 'ink' for the micro-contact printing process. PMSSQ was chosen as it is a cross-linking polymer that exhibits stability 
and robustness at room temperature. The ink was spin-coated on to the PDMS stamp at $3000 \mathrm{rpm}$ for $30 \mathrm{~s}$. The PDMS stamp was then printed on a silicon substrate and peeled off after $10 \mathrm{~s}$ using a pair of tweezers. A very important by-product of this printing procedure is the formation of discontinuities or 'nano-gaps' in the PMSSQ gratings. The origin of these nano-gaps is believed to arise from the mechanical strain $[45,46]$ developed in the thin PMSSQ film (approximately $100 \mathrm{~nm}$ measured using ellipsometry) after the spincoating step (figure 1). Even a slight bending of the elastomeric PDMS stamp produces a tensile strain in the thin PMSSQ film, which then leads to the formation [44] and transfer of the nano-gaps in the printing step, thereby producing nano-gap embedded grating structures. Since the fabrication procedure is done manually (by hand), we can expect a random distribution of the nano-gaps embedded in the gratings varying from stamp to stamp, depending on a myriad of factors such as the force with which the PDMS is peeled off, the direction of peeling as well as the extent of bending of the PDMS stamp [47]. However, the physics of the fluorescence enhancement arising from the resonant coupling of incident light with the evanescent field can be studied well from the random distribution of the nano-gaps in the grating structure.

(a)

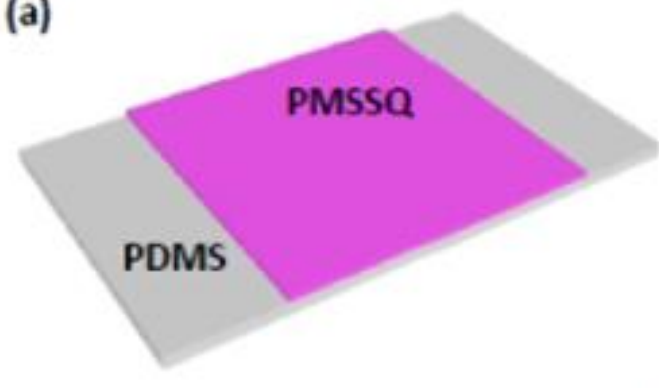

(b)

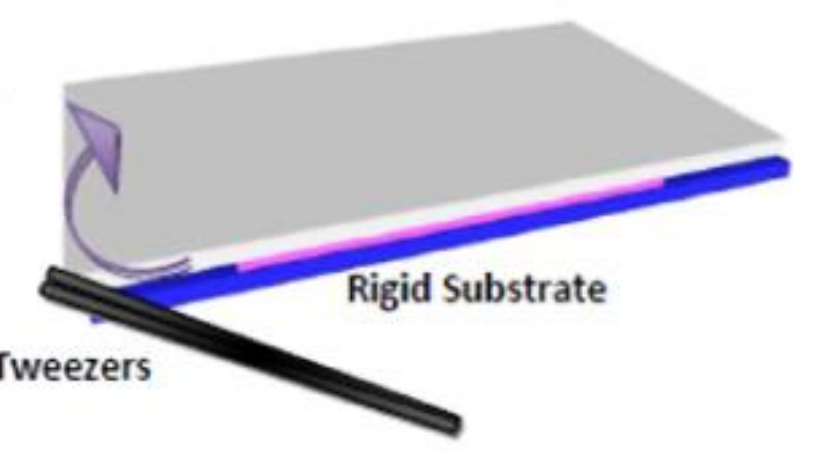

(c) Random formation of nano-gaps

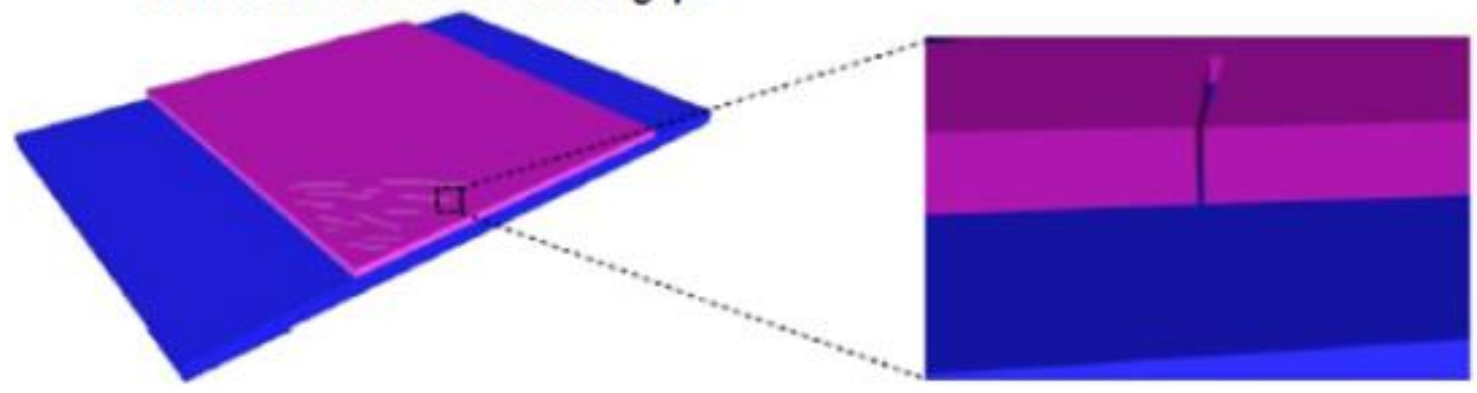

\subsubsection{Figure 4 Nano-gap fabrication}


Figure 1. (a) Spin-coated PMSSQ thin film on a PDMS stamp. (b) $\mu$-contact printing on a glass or silicon substrate followed by peeling of the PDMS stamp using tweezers. (c) The formation of nano-gaps as a result of the tensile strain developed in the film during peel-off. A zoomed-in cross-sectional view of the fabricated nano-gap within the PMSSQ film is shown for clarity.

Following the stamping procedure, the metal depositions were carried out using an AJA RF Magnetron sputtering system. The sputter process was carried out at room temperature, $100 \mathrm{~W}$ RF power, $20 \mathrm{sccm}$ argon flow, and a deposition pressure of 4 mTorr. First, a $2 \mathrm{~nm}$ layer of titanium $(0.2 \AA \mathrm{s}-1)$ was deposited on the substrate, which serves the purpose of giving better adhesion between the silicon and silver. Following this, a $100 \mathrm{~nm}$ layer of silver (100 W RF power, 4 mTorr argon pressure, $1.5 \AA \mathrm{s}-1$ deposition rate) was deposited to complete the fabrication of the plasmonic substrates.

A commercial atomic force microscope-AFM 5500 system (Agilent Technologies) with a vista silicon tip $(\mathrm{k}=40 \mathrm{~N} \mathrm{~m}-1$, resonant frequency $=\sim 295 \mathrm{kHz}$, length $=125 \mu \mathrm{m}$, width $=40 \mu \mathrm{m}$,tip height $=14 \mu \mathrm{m}$,tip radius $\leq 10 \mathrm{~nm}$ ) was used to scan the fabricated samples in tapping mode. To image the nano-gaps embedded within the silver gratings, a Hitachi 4700 FE-SEM (field-emission scanning electron microscope) was used. Images were obtained using an accelerating voltage of $5000 \mathrm{~V}$ and an emission current of $11000 \mathrm{nA}$. Reflectance measurements were performed using a J A Woollam variable angle spectroscopic ellipsometer (VASE). This setup consisted of a Xenon broadband source, which directed light through a monochromator via an optical fiber. To test the fluorescence enhancement from the fabricated nano-gap embedded silver gratings, a thin film of Rhodamine-doped PMSSQ in ethanol $(10 \mu \mathrm{M})$ was deposited on top of the substrates using a spin-coater at $3000 \mathrm{rpm}$ for $30 \mathrm{~s}$ [48]. The film thickness was determined to be $30 \mathrm{~nm}$ and the refractive index to be 1.41 using variable angle spectroscopic ellipsometry measurements.

\subsubsection{Results and discussion}

\section{Surface characterization}

The printed gratings after silver deposition were imaged using the above-mentioned conditions and showed a grating pitch of $\Lambda=401 \pm 5 \mathrm{~nm}$ and a grating height of $\mathrm{h}=64 \pm 3 \mathrm{~nm}$ (figure 2). 


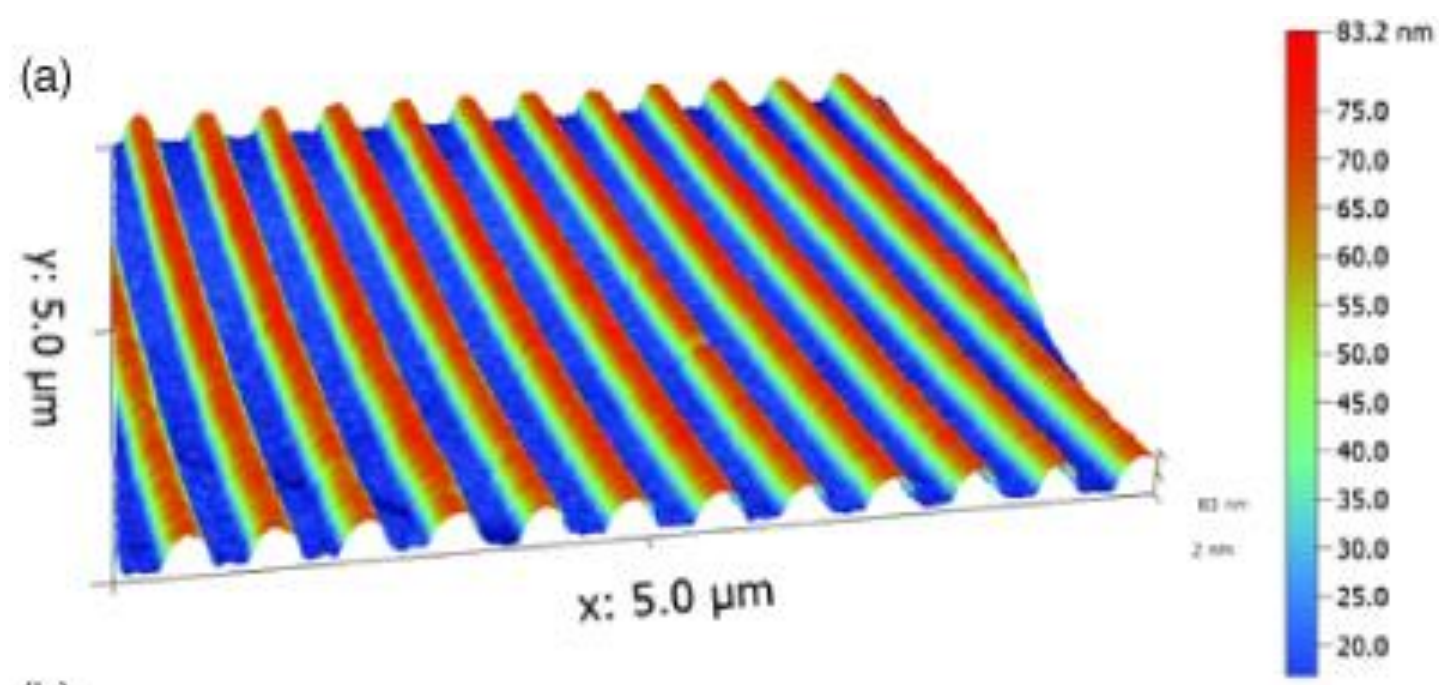

(b)

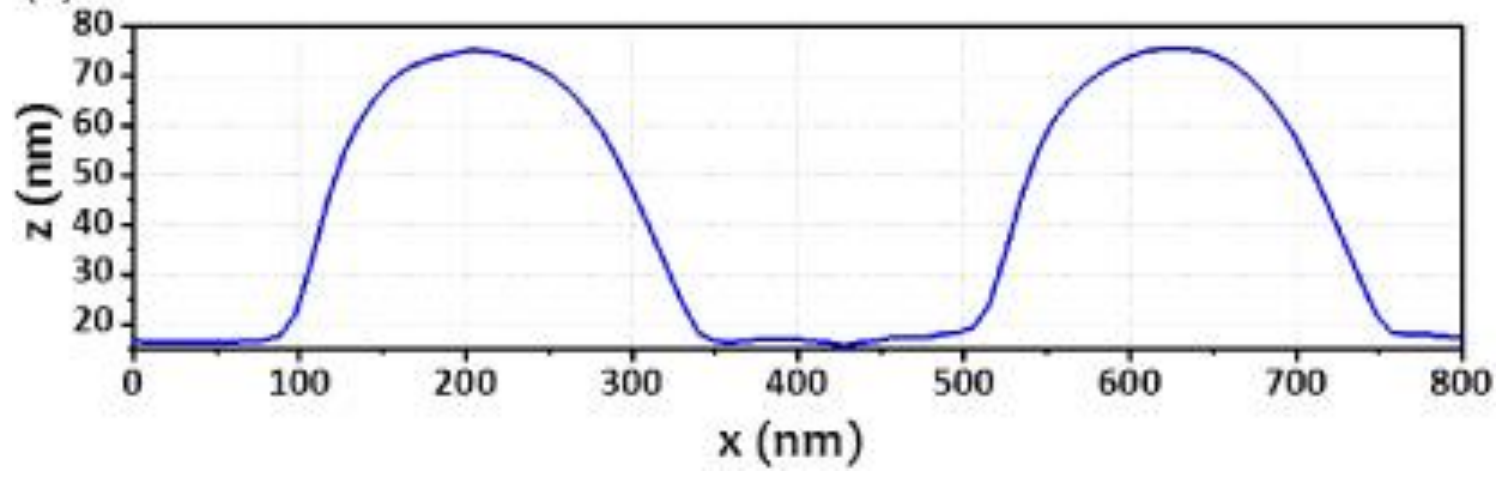

\subsubsection{Figure 5 An atomic force micrograph}

Figure 2. (a) An atomic force micrograph showing the surface topography of the fabricated silver gratings.

(b) Topography of the silver gratings measured using atomic force microscopy, showing a grating height of $65 \mathrm{~nm}$.

Figure 3 shows a top-view scanning electron micrograph of one of the nano-gaps cutting across the periodic silver grating and a lateral width of approximately $30 \mathrm{~nm}$, which is not constant along the nanogap length as it is a randomly formed nano-gap. 
(a)

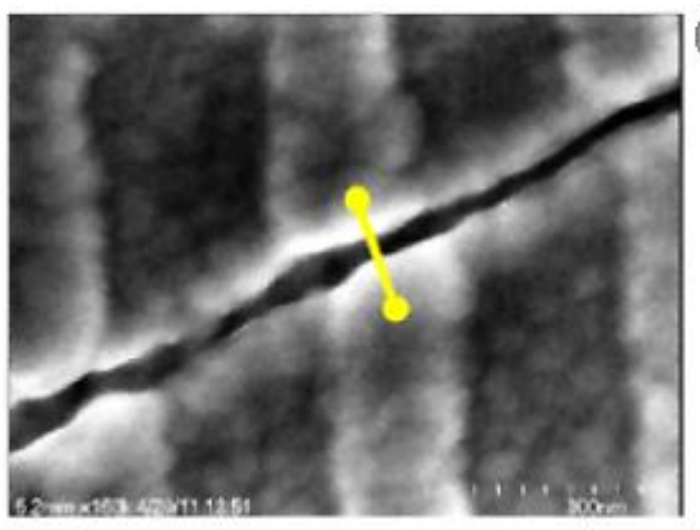

(b)

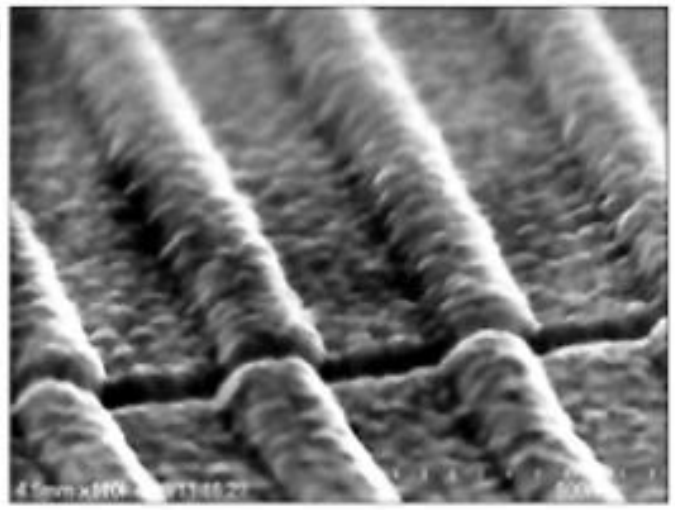

(c)

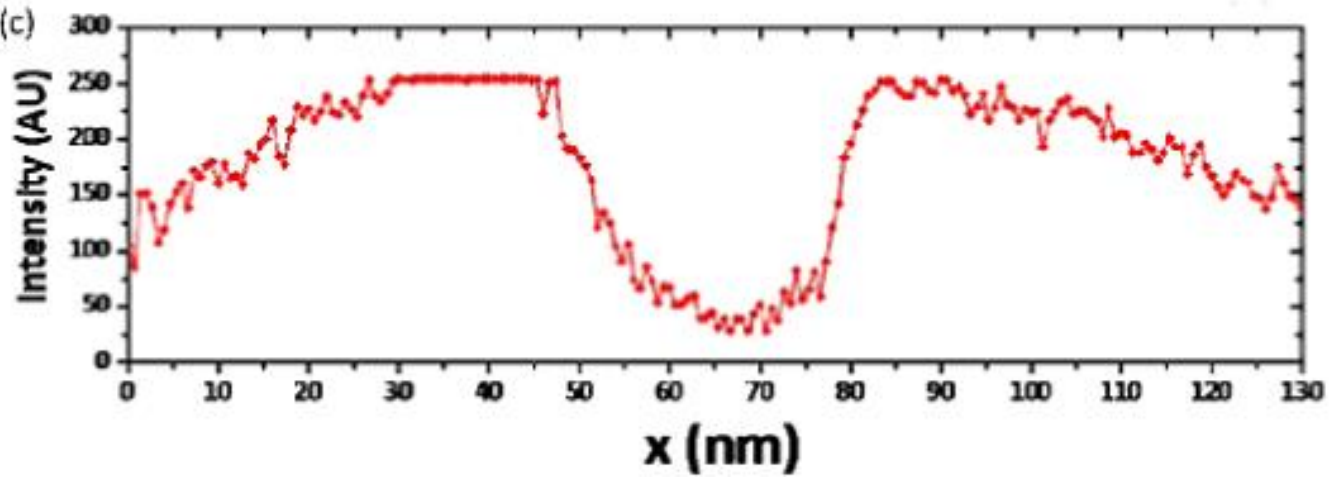

2.4.0 Figure 6 A top-view SEM image of the nano-gap embedded in the silver gratings

Figure 3. (a) A top-view SEM image of the nano-gap embedded in the silver gratings. (b) The two-point profile of the nano-gap along the yellow line shows a nano-gap with an approximate lateral width of $30 \mathrm{~nm}$. (c) A three-dimensional SEM image taken for the nano-gaps cutting across the silver grating, taken at $5 \mathrm{keV}$ accelerating voltage.

\section{Optical characterization}

Reflectance measurements are a standard way for spectral and angular characterization of plasmonic gratings $[49,50]$. The fabricated silver gratings were characterized using a $\mathbf{J}$ A Woollam VASE with air as the surrounding medium. The light was then made incident on the silver grating sample with the gratings aligned perpendicular to the plane of incidence (as mentioned before) for optimum excitation of the surface plasmons on the silver grating. The proper alignment of the grating was made possible by cutting the PDMS stamps in such a fashion that the circular tracks on the HD-DVD were parallel to one of the edges. Since the direction of the circular tracks was known, the gratings were aligned perpendicular to the incoming light as they are parallel to the stamp edge. Only a transverse magnetic (TM) polarized electric field ( $\mathrm{x}-\mathrm{z}$ plane) with a component in the $\mathrm{z}$-direction can generate surface plasmons, since the 
electron oscillations in this plane experience the discontinuity at $\mathrm{z}=0$. Suppose a transverse electric (TE) polarized electric field ( $x-y$ plane) was incident upon the interface, no surface plasmons would be generated since there is no discontinuity in this plane. Hence, surface plasmons modes can only be observed using TM polarization of the incident plane wave [19]. After reflecting from the silver grating substrate, the light reached the detector iris and was then passed on to a Si detector (spectral range: 200$1000 \mathrm{~nm}$ ). The normalized reflectance curves as a function of the incident TM polarized wavelength from 500 to $700 \mathrm{~nm}$ (steps of $1 \mathrm{~nm}$ ) for six different incident angles were obtained (figure 4). The resonance wavelength is characterized by a sharp minimum in the reflectance curves, indicating the localization of the electric field at the silver-air interface. Besides the sharp reflectance dip, which denotes the resonance wavelength for a specific angle of incidence, we observe a cusp that appears for slightly lower wavelengths, where the reflectance value drops as well. At the cusp, one of the diffracted orders has a diffraction angle of $90^{\circ}$, which leads to a lower value of reflectivity in that region [51]. The value of $\theta$ SPR for $495 \mathrm{~nm}$ TM polarized light can be calculated to be $8^{\circ}$ by linear extrapolation of the measured reflectance plots (figure 4 inset). This value agrees very well with the resonance angle calculated using equation (1). Similarly, the resonance angle for different wavelengths can be calculated from the linear fit obtained in figure 4 . 


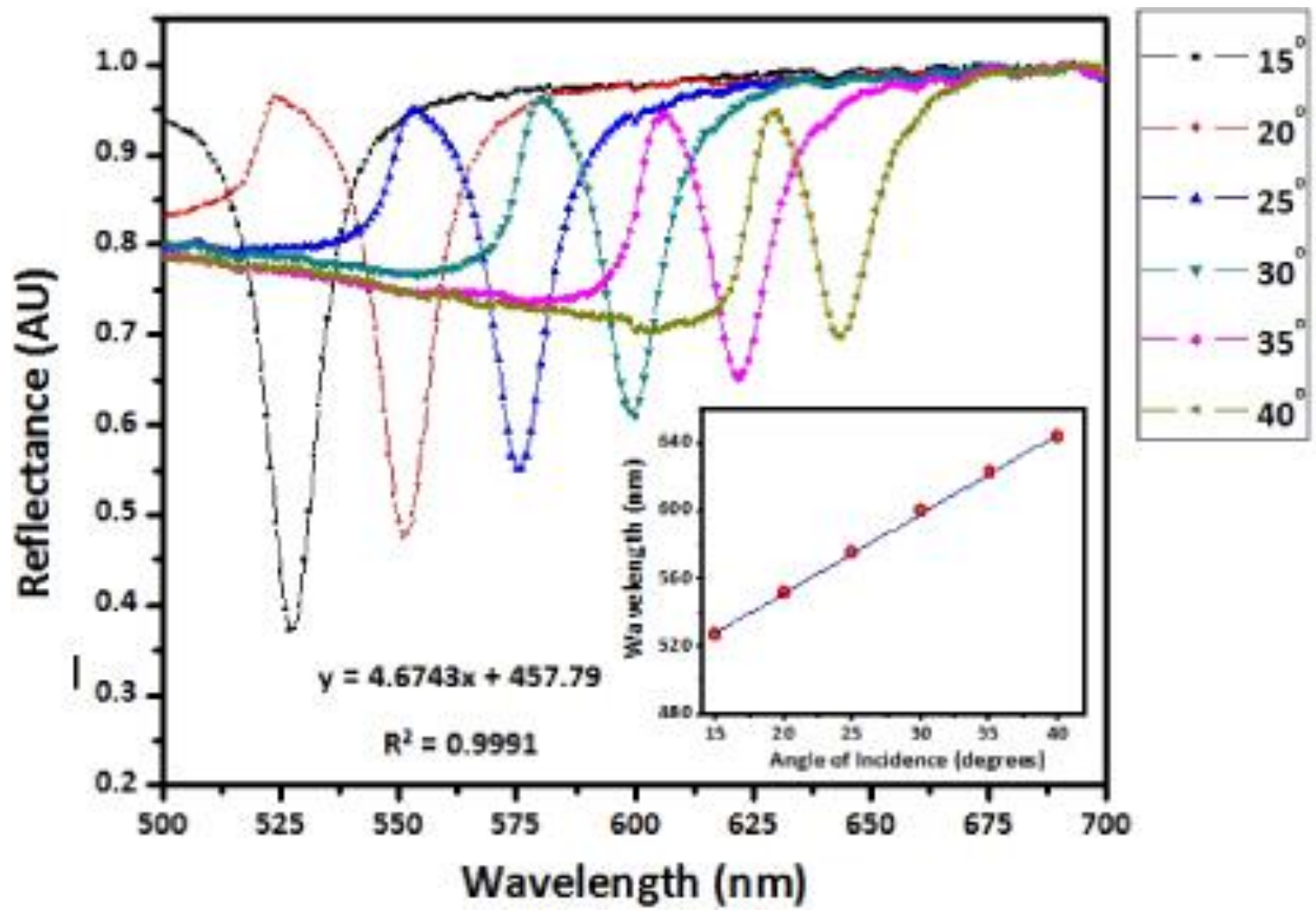

\subsubsection{Figure 7 Measured reflectance vs wavelength}

Figure 4. Measured reflectance spectra for the fabricated silver gratings as a function of wavelength for six different incident angles: $\theta_{\mathrm{inc}}=15^{\circ}, 20^{\circ}, 25^{\circ}, 30^{\circ}, 35^{\circ}$, and $40^{\circ}$. The SPR angle $\left(\theta_{\mathrm{SPR}}\right)$ is denoted by the sharp dip in reflected intensity at the minima. Inset: linear extrapolation of the measured data to calculate that $\theta_{\mathrm{SPR}}=8^{\circ}$ for $495 \mathrm{~nm}$ transverse magnetic light.

\section{Silver gratings FDTD simulations}

It is imperative to realize the near-field electromagnetic distributions to compare the experimental results of fluorescence enhancement to electromagnetic field enhancement from the modeled simulations of the nano-gap embedded plasmonic gratings. For this purpose, electromagnetic field computations were performed using commercially purchased software-FullWAVE (RSoft Inc.) based on the finite difference time domain (FDTD) algorithm [52-54]. The first configuration used for field simulations was a silver grating with a semi-circular shape, a grating pitch of $\Lambda=400 \mathrm{~nm}$, a grating height of $\mathrm{h}=65 \mathrm{~nm}$, and surrounding dielectric as air, which is very similar to the topography obtained using AFM. A rectangular launch field was described with unit power at $\mathrm{z}=0.35 \mu \mathrm{m}$ and the angle of incidence could be varied. A monitor was placed above the launch field at $\mathrm{z}=0.38 \mu \mathrm{m}$, measuring the received power and averaging it 
over time to provide the final output. This monitor could be thought of as a detector that measures reflectance. The computational domain was defined from $\mathrm{x}=-0.2$ to $+0.2 \mu \mathrm{m}$ and $\mathrm{z}=0$ to $0.4 \mu \mathrm{m}$. A periodic boundary condition was used in the $\mathrm{x}$-direction since the grating is periodic in this direction. For the maximum $\mathrm{z}$ value, a perfectly matched layer (PML) boundary condition was used, while the minimum $\mathrm{Z}$ value used a perfect electric conductor (PEC) boundary condition, as this boundary is inside the silver surface and can be regarded as a perfect electrical conductor. The grid size was uniform and equal in both directions and chosen to be $\Delta \mathrm{x}=\Delta \mathrm{z}=0.005 \mu \mathrm{m}$. The FDTD stop time was set to $40 \mu \mathrm{m}$ (in units of cT) and a continuous wave $(\mathrm{CW})$ excitation was chosen to be incident upon the silver structure. The reflected power was detected using the monitor and plotted as a function of the angle of incidence (varying from $0^{\circ}$ to $18^{\circ}$; steps of $0.01^{\circ}$ ) for an excitation wavelength of $495 \mathrm{~nm}$ and TM polarized light (E-field in the $\mathrm{x}-\mathrm{z}$ plane). Figure 5 shows the reflectance as a function of the angle of incidence for the above simulation parameters. It is clear that, at an incidence angle of $8^{\circ}$, the reflectance drops to a minimum, which is characteristic of the SPR dip as seen in silver gratings. Hence, this structure simulates the fabricated samples very closely, as the silver plasmonic gratings also showed a $\theta \mathrm{SPR}=8^{\circ}$ for $495 \mathrm{~nm} \mathrm{TM}$ polarized light in air, as shown previously. 


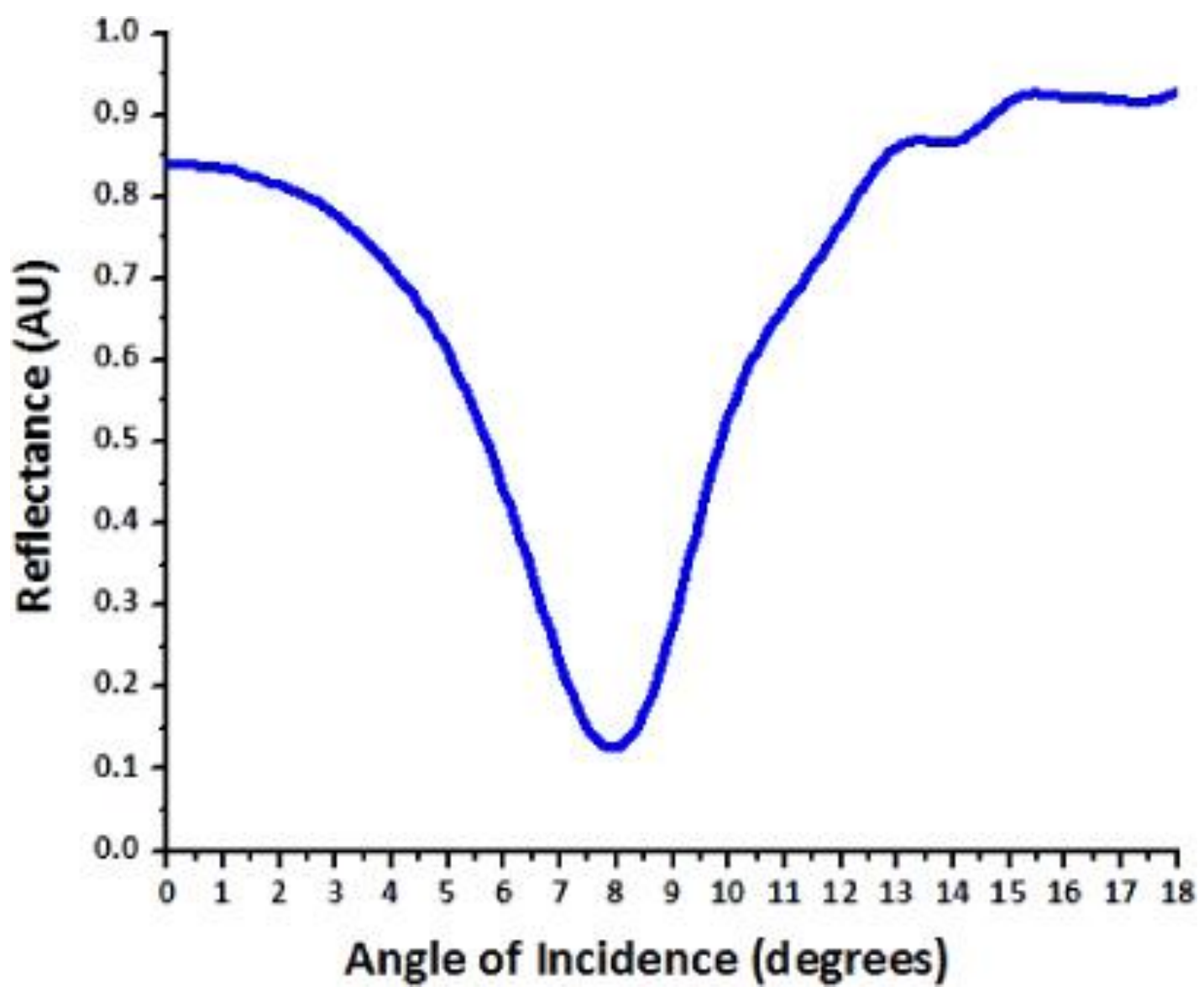

\subsubsection{Figure 8 Reflectance vs Angle of incidence}

Figure 5. Reflected power as measured by the monitor as a function of the angle of incidence. The SPR angle is denoted by the sharp dip in the reflectance value at $8^{\circ}$ for $495 \mathrm{TM}$ polarized light. This angle denotes the optimal coupling for the incident photons to the grating surface at a specific wavelength.

To map the near-field intensity distributions for the silver-air interface, another monitor was placed in the $x-z$ plane; this measured the magnetic field going out of the plane $\left(\boldsymbol{H}_{y}\right)$ for the TM polarized $495 \mathrm{~nm}$ light. In figure $\underline{6}$, three different excitation angles are shown, and it is evident that the maximum field intensity $\left(H_{y}^{2}\right)$ is seen at $\theta_{\mathrm{SPR}}=8^{\circ}$ as opposed to $0^{\circ}$ or $18^{\circ}$ incidence.
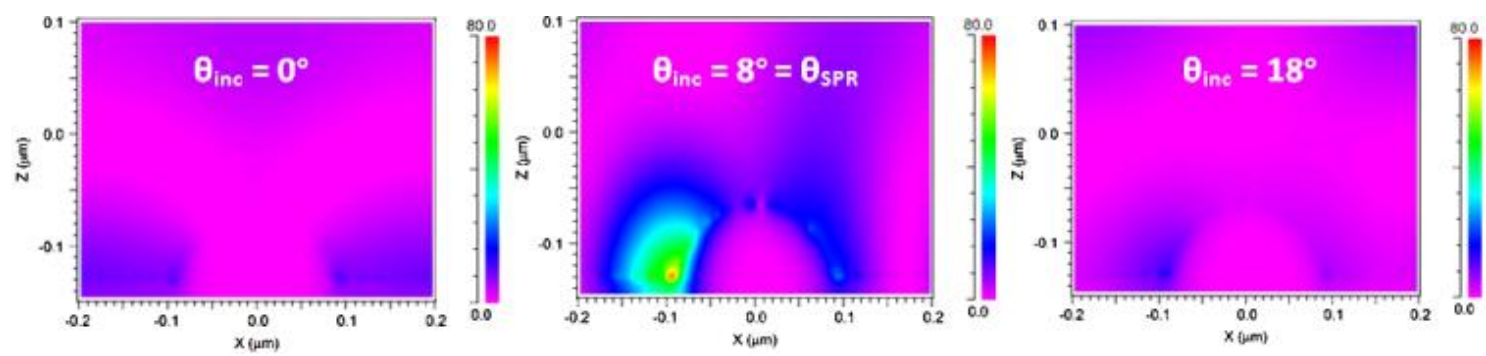


\subsubsection{Figure 9 Field intensity distributions}

Figure 6. Field intensity distributions $\left(H_{y}^{2} / H_{y 0}^{2}\right)$ for three different angles of incidence with $495 \mathrm{~nm}$ TM polarized light. An angle of $8^{\circ}$ provides the maximum field enhancement at the grating surface, while moving away from the SPR angle leads to lesser enhancement and a lower field concentration.

The field intensity was also plotted as a function of the distance into the dielectric $(z)$ from the silver grating surface at $x=-0.1 \mu \mathrm{m}$ (figure 7) and shows an evanescent profile for the SPR case. The maximum intensity is achieved at the silver-air interface and exponentially decreases as it moves into the air. The intensity reaches $1 / e(37 \%)$ of its maximum value at a distance of $\frac{1}{k_{Z} \prime}=47 \mathrm{~nm}$ away from the interface, which is also known as the 'decay length'.

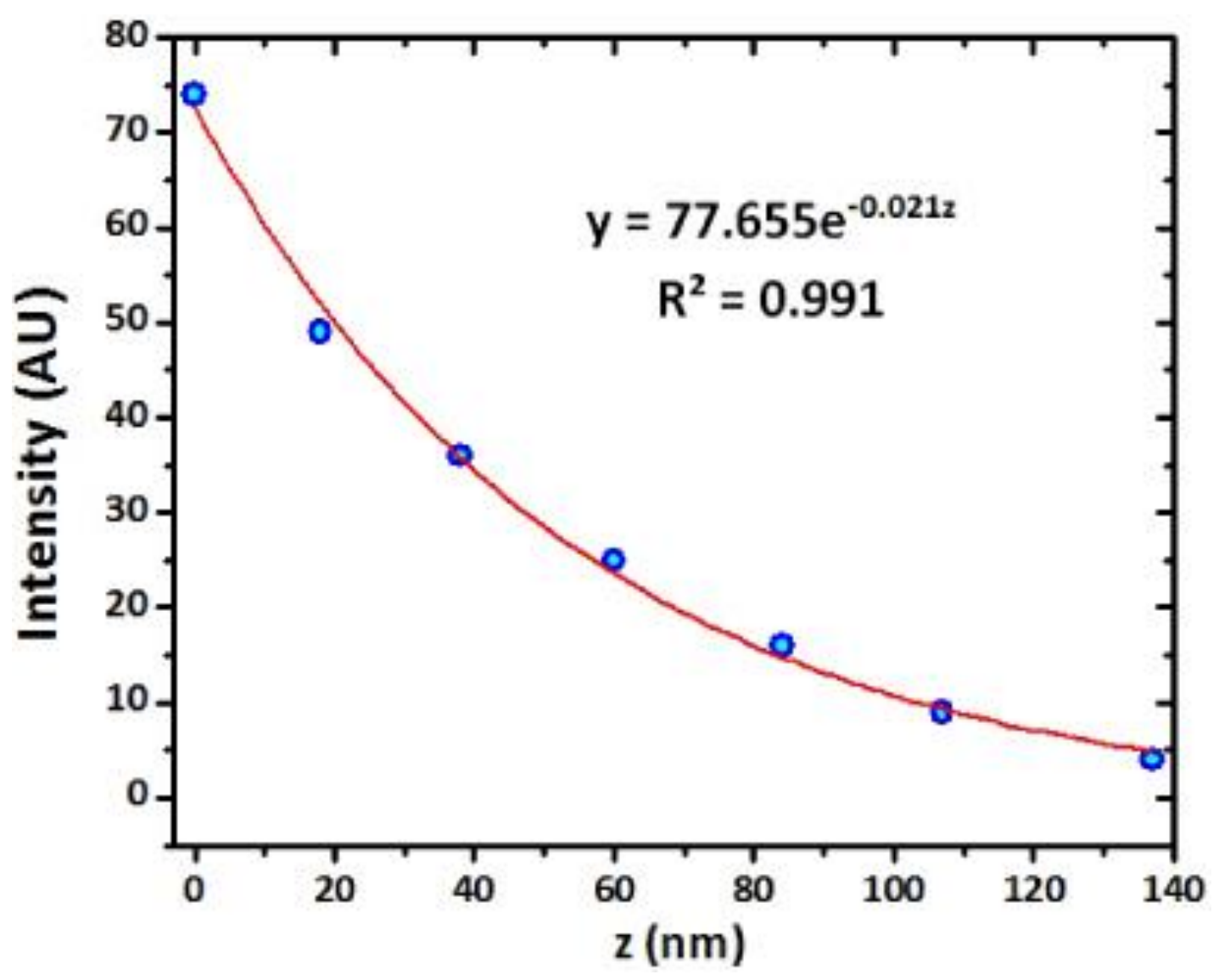

\subsubsection{Figure 10 Field intensity as a function of the distance}

Figure 7. The field intensity as a function of the distance away from the metal-air interface, showing an exponential decay as expected for surface plasmon resonance and a $k_{z}{ }^{\prime \prime}=0.021 \mathrm{~nm}^{-1}$. 
Based on the field intensity obtained above and the quenching behavior of a metal surface, the optimal fluorophore placement can be chosen by taking these two competing phenomena into account. The emission efficiency $\left(\eta_{\mathrm{em}}\right)$ of the dye in this case is given by the following equation [55]:

$\eta_{\mathrm{em}}(d)=\frac{I(d)}{I_{\infty}}=\left[1+\left(\frac{d_{\mathrm{F}}}{d}\right)^{4}\right]^{-1}$

where $d$ is the distance between the metal and the fluorophore, $I(d)$ is the emission intensity at a finite distance $d, d_{\mathrm{F}}$ is the Förster radius, and $I_{\infty}$ is the emission intensity where the fluorophore will not interact with the metal. The Förster radius is defined as the separation between the metal surface and the fluorophore where the emission efficiency is 0.5 . Figure $\underline{8}$ shows the normalized field intensity of the evanescent surface plasmon on the silver gratings, the emission efficiency of a dye in the presence of a fluorophore (refer to equation (2)), and the product of both these curves. From this curve, one can see that the optimal fluorophore to grating distance, $d_{\mathrm{opt}}$, is $11 \mathrm{~nm}$ for a dye with Förster radius, $d_{\mathrm{F}}=5 \mathrm{~nm}$.

(a)

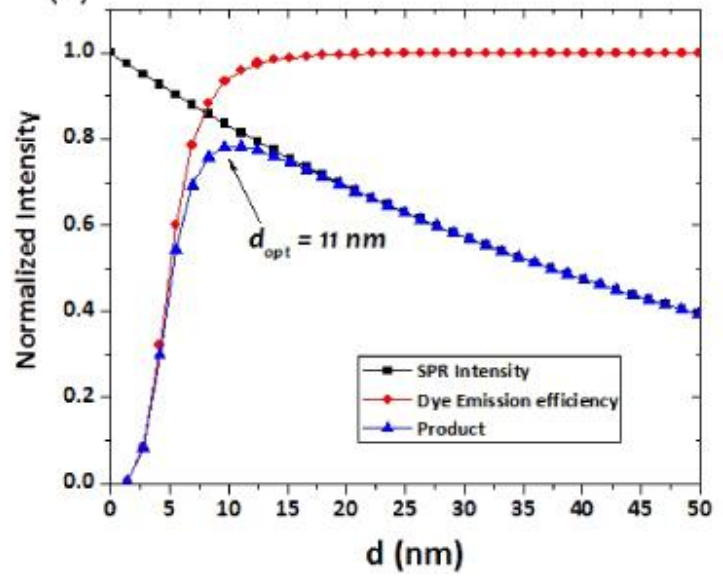

(b)

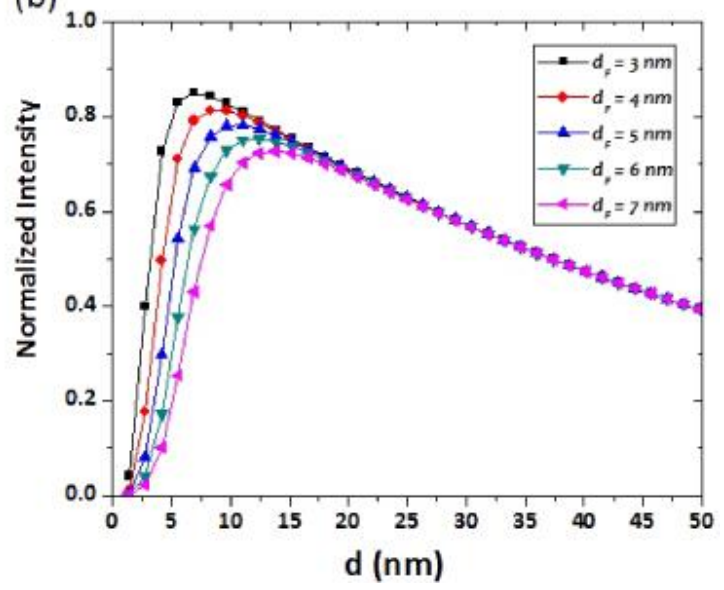

\subsubsection{Figure 11 Normalized SPR intensity at resonance}

Figure 8. (a) Normalized SPR intensity at resonance and fluorophore emission intensity as a function of the distance between the fluorophore and metal. The optimal separation is $11 \mathrm{~nm}$ for a Förster radius of $5 \mathrm{~nm}$, and the maximum efficiency also decreases with increasing $d_{\mathrm{opt}}$, as shown in (b). The optimal distance also changes as a function of the Förster radius and it can be seen that the maximum normalized intensity also decreases with increasing Förster radius [두, 57].

\section{Nano-gap embedded silver gratings FDTD simulations}


Since the nano-gap is of finite length, we cannot use the periodic boundary conditions anymore and will have to use PML in the $x$-direction. The new structure created is shown in figure $\underline{9}$, where the nano-gap with a lateral width of $20 \mathrm{~nm}$ (shown by SEM measurements previously) cuts across a silver grating structure with 21 periods. The computational domain was defined from $x=-4.2$ to $+4.2 \mu \mathrm{m}$ (PML boundary condition at both boundaries), $y=0.03 \mu \mathrm{m}$ (PEC) to $0.3 \mu \mathrm{m}$ (PML), and $z=-2.5$ to $+2.5 \mu \mathrm{m}$ (PML at both boundaries). The grid size for this three-dimensional computational domain was selected to be $\Delta x=\Delta y=\Delta z=0.01 \mu \mathrm{m}$. The FDTD stop time was set to $40 \mu \mathrm{m}$ (in units of cT) and a continuous wave (CW) excitation was chosen to be incident upon this nanostructure.
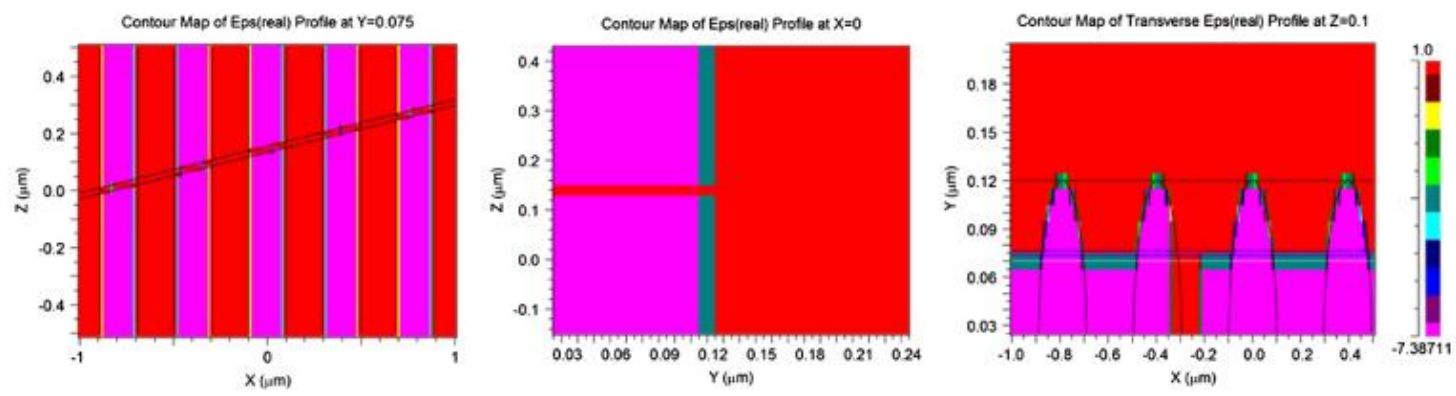

\subsubsection{Figure 12 Contour profile of the nano-gap embedded in silver gratings}

Figure 9. Contour profile of the nano-gap embedded in silver gratings in three different planes.

To choose the number of periods necessary to get a plasmonic interaction from the designed configuration, a convergence study was performed by varying the number of periods, $\mathrm{Np}$, from 1 to 29 (see supporting information available at stacks.iop.org/Nano/23/495201/mmedia). In the interest of keeping the computational domain smaller to avoid unrealistic simulation times, $\mathrm{Np}=21$ was chosen to be the optimal number to see the surface plasmon resonance from the silver gratings.

To see the field intensity distribution at the intersection of the nano-gap with the silver gratings, a spatial monitor was placed at $\mathrm{y}=0.08 \mu \mathrm{m}$. The excitation conditions were chosen as $495 \mathrm{~nm}$ TM polarized light for an incidence angle of $8^{\circ}$, to achieve surface plasmon resonance on the silver gratings. For the same optical launch conditions, three different configurations (figure 9) were compared: a nano-gap with the gratings, the gratings without a nano-gap, and a nano-gap on a flat film. The intensity values obtained from the monitor placed at $\mathrm{y}=0.08 \mu \mathrm{m}$, i.e., at the silver surface, for the different configurations in figure 10 are listed in table 1 . The intensity values listed are for the point $(0,0)$ in the field distribution maps. This demonstrates that the field enhancement in this case is due to the combined effect of grating coupled 
surface plasmon resonance (GC-SPR) and localized surface plasmon resonance, whereas in the case of silver gratings without nano-gaps the field enhancement is due only to the GC-SPR, and in the metallic nano-gap on a flat surface the enhancement is entirely due to a localized SPR phenomenon. The fluorescence enhancement characteristics of the nano-gap embedded gratings and their comparison to other plasmonic structures are discussed in section 3.5.

(a)

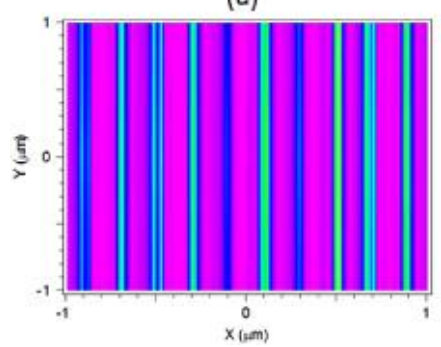

(b)

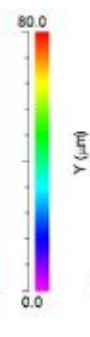

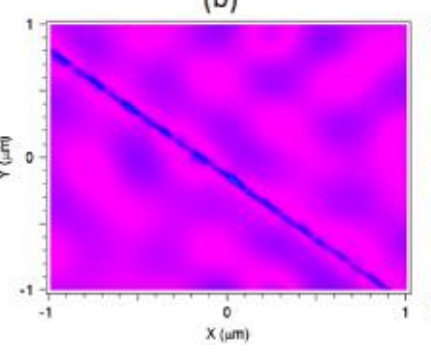

(c)

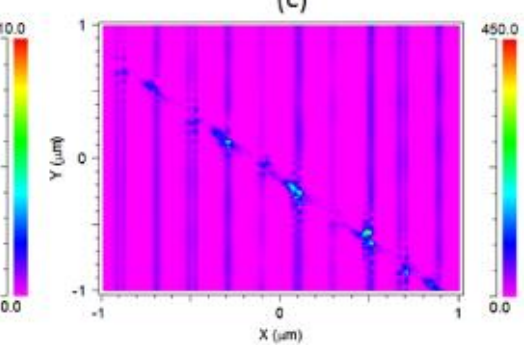

\subsubsection{Figure 13 Field intensity distributions at the air-silver interface}

Figure 10. Field intensity distributions at the air-silver interface (top-view) for $495 \mathrm{~nm}$ TM polarized light

(a) silver grating, (b) silver nano-gap and (c) nano-gap embedded in silver grating.

Table 1. Field intensity values for the different configurations at point $(0,0)$ under the same optical excitation conditions as shown in figure $\underline{10}$.

\begin{tabular}{ll}
\hline & Intensity \\
\hline Flat silver film & 1 \\
Nano-gap on flat silver & 3 \\
Silver grating & 52 \\
Nano-gap embedded in silver grating & 299 \\
\hline
\end{tabular}

\subsubsection{Table 1 Field intensity values}

Table 1. Field intensity values for the different configurations at point $(0,0)$ under the same optical excitation conditions as shown in figure $\underline{10}$.

\section{Fluorescence studies}

Plasmonic gratings have been used extensively to excite the fluorophores present in the immediate vicinity of the evanescent field and achieve better limits of detection and improved imaging contrast in fluorescence microscopy $[58,59]$. The combination of nano-gaps embedded in the plasmonic grating structure provides much higher levels of near-field concentration due to the combination of localized SPR and propagating surface plasmon polaritons, as discussed above. The evanescent field produced in the 
nano-gap embedded gratings is much stronger than the gratings alone. Therefore, we can expect much higher emission intensities from fluorophores present at the intersection of a nano-gap with the plasmonic grating, as opposed to a grating without the presence of nano-gaps or just nano-gaps on a flat silver film. Fluorescence studies were performed using a traditional epi-fluorescence microscope setup in which light illuminates the sample in the form of a cone of excitation, which contains all angles from $0^{\circ}$ to a maximum angle $\alpha$, given by the following equation:

$\mathrm{NA}=n \sin \alpha$

where NA is the numerical aperture of the objective lens used, $n$ is the refractive index of the medium between the sample and the objective, and $\alpha$ is the maximum incident angle [60,61]. For a $10 \times$ objective lens with an NA of 0.3 and air surrounding the sample, the maximum incident angle is $17.5^{\circ}$, which is greater than the $\theta \mathrm{SPR}=8^{\circ}$ of the silver gratings in air for $495 \mathrm{~nm}$ light as shown previously via reflectance measurements. Hence, the surface plasmons on the silver gratings can be excited using just a regular microscope setup, as the $\theta \mathrm{SPR}$ is contained within the cone of excitation of a $10 \times$ objective, which includes both TM as well as TE polarization states. Additionally, as seen in figure 11, the absorption peak of Rhodamine 6G (the dye used in this study) is centered on $535 \mathrm{~nm}$ and is ideal to be used with a 
fluorescein isothiocyanate (FITC) filter cube set, whose optical components are shown in the same figure.

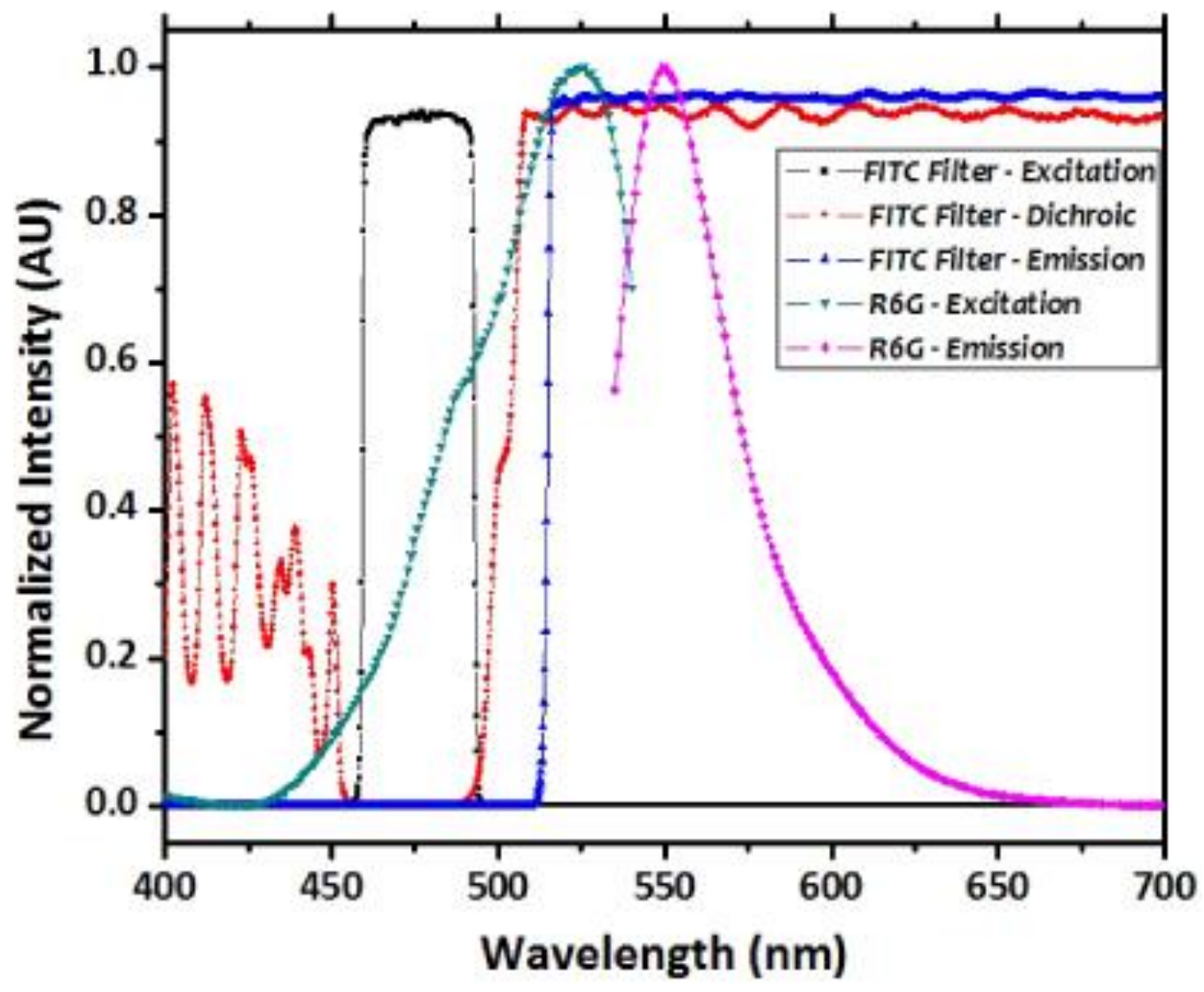

\subsubsection{Figure 14 The excitation and emission spectra of Rhodamine 590 (R6G)}

Figure 11. The excitation and emission spectra of commercially available Rhodamine 590 (R6G) along with the optical components of the FITC filter cube set used for measuring fluorescence.

The plasmonic substrates after the fluorophore film deposition were imaged with an epifluorescence microscope (Olympus BX51WI) equipped with a $10 \times$ objective $(\mathrm{NA}=0.3)$, a FITC filter cube (excitation: 460-495 nm; emission: >516 nm), a $300 \mathrm{~W}$ Xenon broadband white light source, and a high-resolution detector (ORCA-Flash 2.8 CMOS Camera, Hamamatsu). The fluorescence micrograph shown in figure $12(\mathrm{a})$, obtained using a $10 \times$ objective $(\mathrm{NA}=0.3)$, displays a clear fluorescence enhancement at the nano-gaps when compared to the surrounding grating region. 

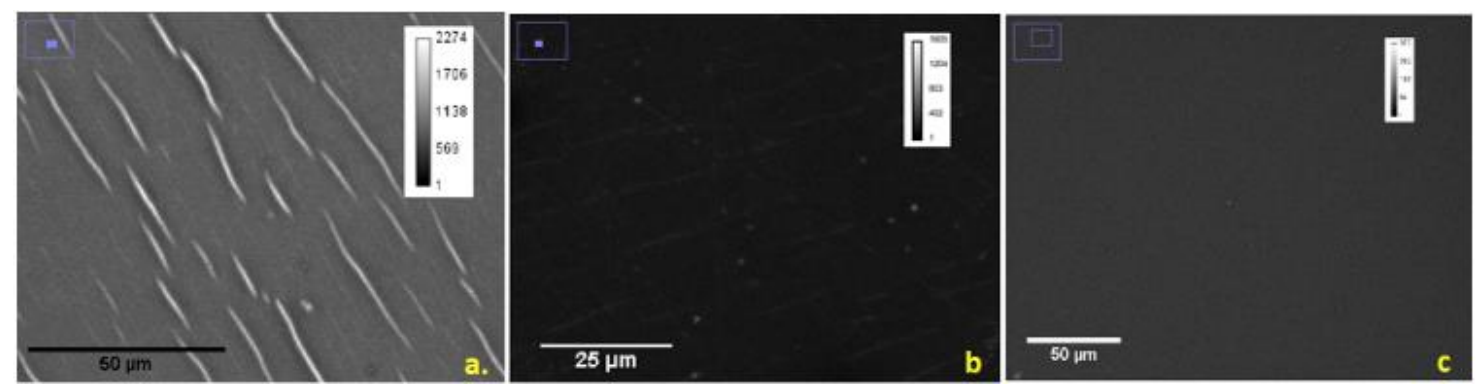

\subsubsection{Figure 15 Fluorescence micrographs}

Figure 12. Fluorescence micrographs of (a) 'nano-gaps' embedded in gratings, (b) 'nano-gaps' on flat silver and (c) glass slide taken with a $10 \times$ objective and a FITC filter and a $6 \%$ ND filter and 60 ms exposure time of the CMOS detector. The intensity inside the nano-gap embedded gratings is much brighter than for nano-gaps on a flat film for the exact same fluorophore thin film on the surface.

The fluorescence enhancement factors (EF) for the different substrates with respect to bare glass slides were calculated using the following equation:

$\mathrm{EF}=\frac{I_{n}-I_{n B}}{I_{f}-I_{B}}$

where In is the fluorescence intensity with the dye layer on the plasmonic substrate, InB is the intensity without the dye on the plasmonic substrate, and If and IB are the intensities with the dye and without the dye layers on glass, respectively. Fluorescence image analysis was performed using 'ImageJ' to measure the average and maximum intensity from the nano-gap regions. The performance of this substrate was evaluated by comparing the fluorescence intensities with other substrates, including nano-gaps on a flat silver surface, silver gratings with no nano-gaps, flat silver film and a transparent glass slide. As the quantum yield of Rhodamine 6G dye is inherently high (>95\%), the fluorescence enhancements observed upon the plasmonic substrates may be attributed solely to enhanced absorption of the incident light due to the extreme field localization enabled by the substrate architecture. To reduce the intensity of the incident light on the samples to prevent photobleaching, a neutral density filter, U-25ND6 (6\% transmission), was used.

Fluorescence data analysis was performed for the various micrographs obtained using the filter setup described above (see supporting information available at stacks.iop.org/Nano/23/495201/mmedia). Figure 13(a) shows the frequency distribution of the maximum enhancement factor, which is calculated 
using the highest pixel intensity within a nano-gap enclosed region in the fluorescence micrograph. Figure 13(b) shows the frequency distribution of the mean enhancement factor, which is calculated using the average pixel intensity within a nano-gap enclosed region in the fluorescence micrograph. The nano-gaps on flat silver films showed an average enhancement of 9 times when compared to a glass slide due to the excitation of localized surface plasmons [62]. The silver grating by itself provides almost a 25 -fold enhancement factor when compared to glass. An average enhancement factor of 68 times (figure 13(d)) and a maximum enhancement of 118 times (figure 13(c)) was observed for the nano-gaps embedded in the gratings with respect to a microscope glass slide. It is evident that the nano-gap embedded gratings show a much higher enhancement than the nano-gaps on a flat silver surface that were prepared by using a flat PDMS stamp instead of a periodically patterned stamp. This effect can be attributed to the presence of propagating plasmons on the silver grating surface as opposed to no plasmonic coupling on the flat silver films. The combination of both the localized and propagating surface plasmons yields the maximum enhancement effect in this scenario. 

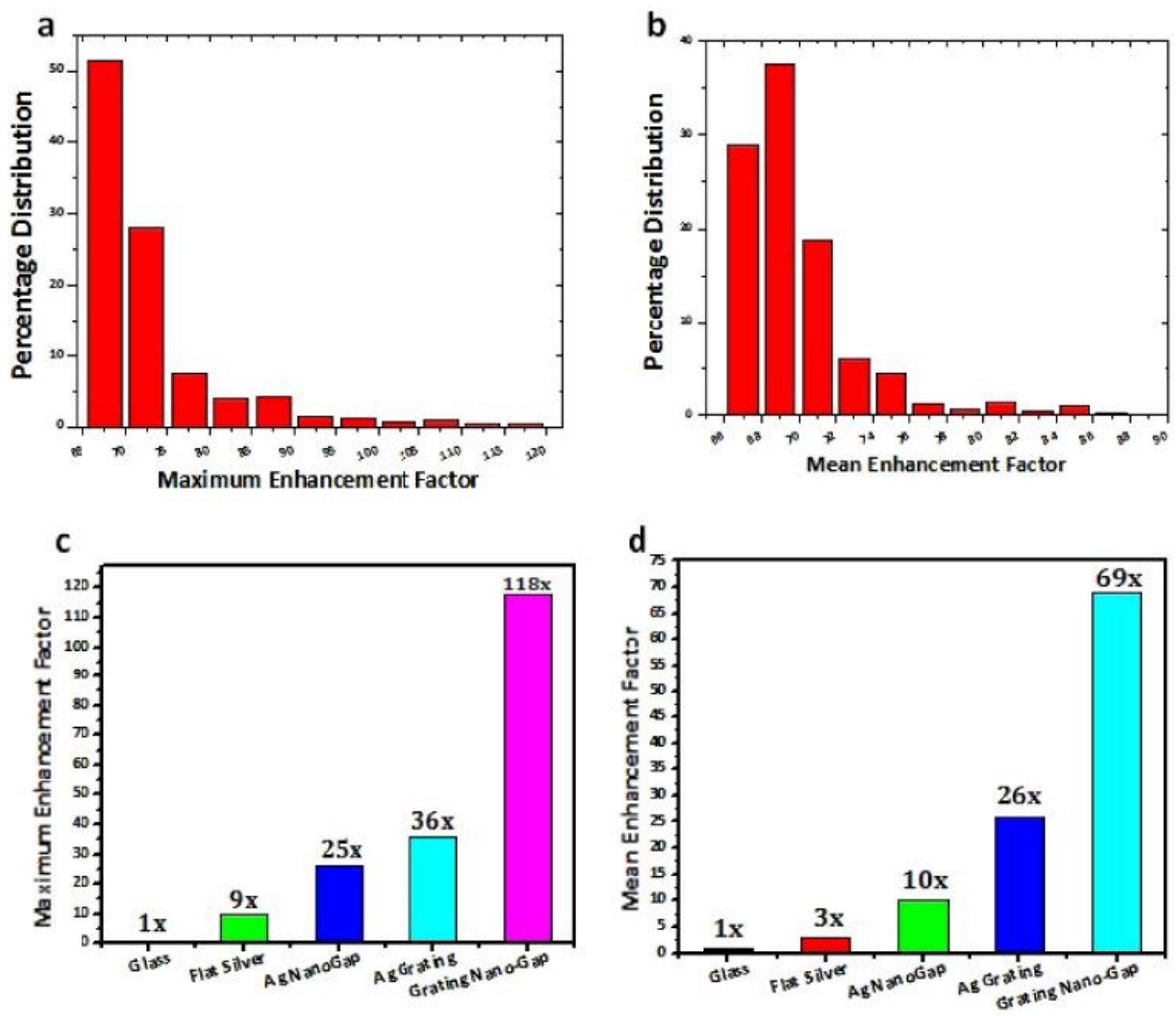

\subsubsection{Figure 16 Enhancement factors}

Figure 13. Percentage distribution for (a) the maximum enhancement factor and (b) the mean enhancement factor for Rhodamine fluorescence in the case of nano-gaps embedded in silver gratings. Comparison of the fluorescence enhancement for different substrates with respect to a regular microscope glass slide for (c) maximum enhancement factor and (d) mean enhancement factor.

The nano-gap embedded gratings with the thin fluorophore layer were also imaged using SEM as well as an optical microscope for a bright-field image. Figure 14 shows the images of the exact same area imaged using three different techniques, which agree well with each other. The bright-field image shows the nano-gaps as dark features in a white background of the reflected silver gratings. The SEM image also provides leverage to the fact the extraordinary fluorescence enhancement seen in these samples is due to 
the presence of very narrow nano-gaps, which are on the order of $20-30 \mathrm{~nm}$ in terms of their lateral width.
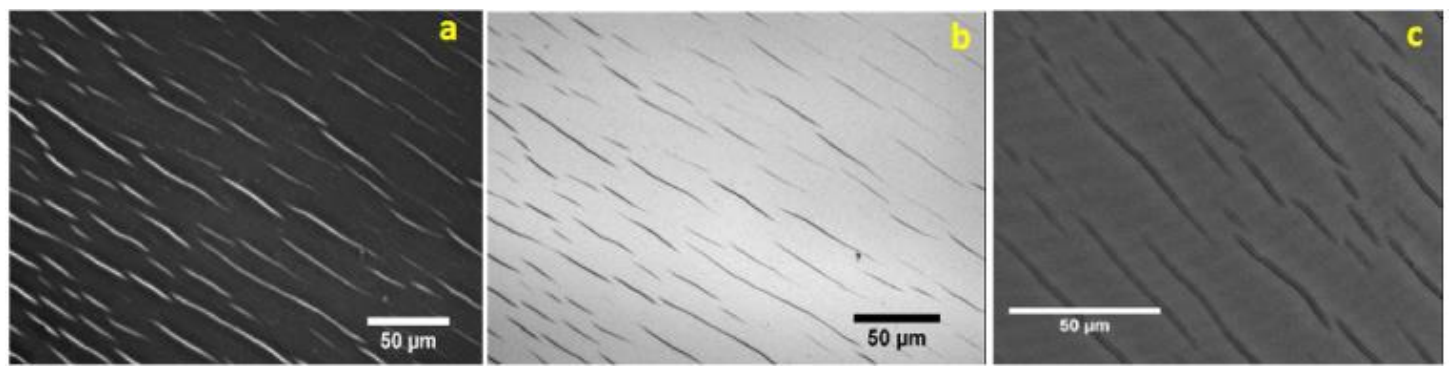

\subsubsection{Figure 17 Fluorescence micrographs}

Figure 14. (a) Fluorescence micrograph of the nano-gap embedded gratings with a thin Rhodamine-doped film on top using a $40 \times$ objective and the FITC filter set. (b) Bright-field image of the exact same area using a $40 \times$ objective. (c) SEM image of the same area as in (a) and (b) showing the actual physical dimensions of the nano-gaps.

A $100 \times$ oil-immersion objective (Olympus-UPlanApo, NA $=1.3$ ) was also used to image the fluorescence from the nano-gap embedded silver gratings. Figure 15(a) shows a very high-resolution image depicting the silver gratings and the nano-gap cutting across them at a random angle. The points of extraordinary fluorescence intensity occur at the intersection of the nano-gap with the grating pattern, due to the extreme field enhancement at these points, which is expected from the FDTD simulation results shown earlier. The scanning electron micrograph of one of the ultra-fluorescent nano-gaps embedded in the silver grating pattern with the thin Rhodamine-doped film deposited on top (figure 15(b)) shows a nano-gap lateral width of approximately $20 \mathrm{~nm}$.
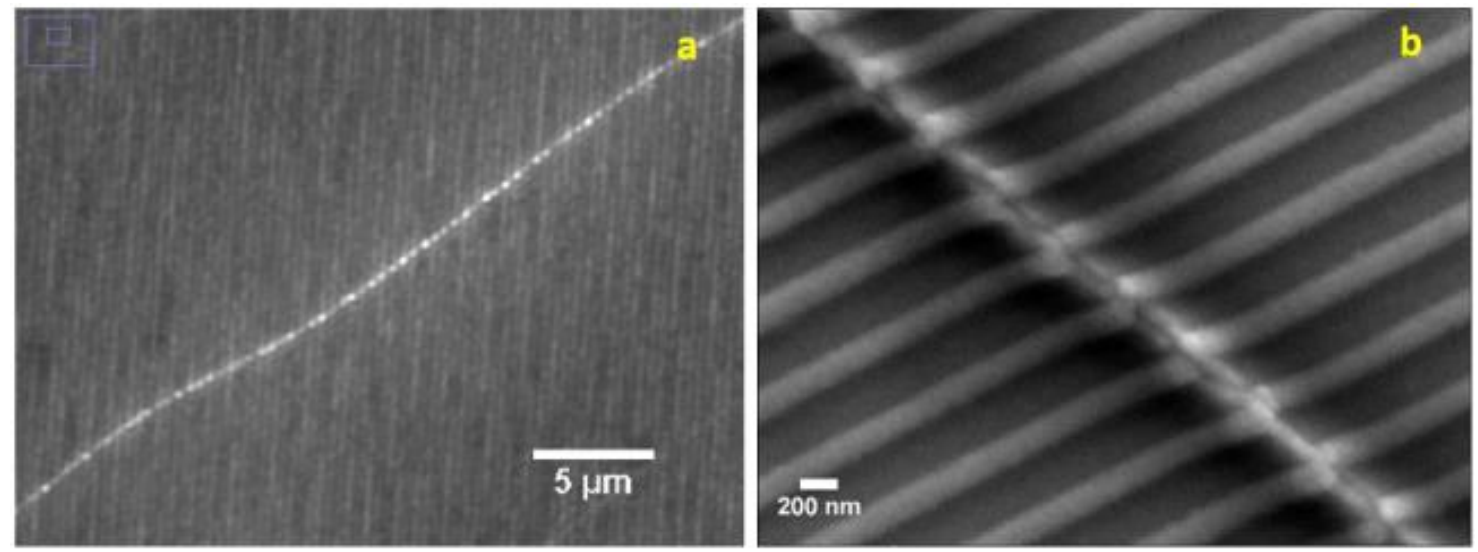

\subsubsection{Figure 18 Fluorescence micrographs}


Figure 15. (a) Fluorescence micrograph of the nano-gap embedded gratings with a thin Rhodamine-doped film on top using a $100 \times$ oil-immersion objective and the FITC filter set. (b) SEM micrograph of the nanogaps embedded in the gratings with the Rhodamine film on top, showing a lateral width of approximately $20 \mathrm{~nm}$.

From the fluorescence studies, it is evident that the nano-gaps embedded in gratings have much higher field intensities when compared to the gratings and the nano-gaps on a flat silver film under resonance conditions. However, these fluorescence enhancement factors are much lower compared to the field enhancement of 300 times observed using FDTD simulations for the nano-gap embedded gratings and field enhancement of 52 times for the silver gratings. This discrepancy comes from the fact that fluorescence enhancement depends not only on the electric field enhancement due to the plasmonic structure resonance, but is also heavily dependent on the optical characteristics of the fluorophore in the vicinity of the metal surface. In this case of thin fluorophore films on a plasmonic grating surface around 30 $\mathrm{nm}$ thick, the first $10 \mathrm{~nm}$ of the dye film will experience considerable quenching [63, 64], where as the top $20 \mathrm{~nm}$ will see the full effect of the evanescent field enhancement, as shown in section 3.4. Other possible reasons for the disagreement between the simulated enhancement factors in section 3.4 and the measured enhancement factors are as follows: (i) light illuminates the samples in the form of a cone of light containing not only the resonance angle but also other angles, leading to the observation of an average enhanced effect, whereas the theoretical enhancement factors are a result of specific illumination at the resonance angle of incidence. (ii) The use of p-polarized light in the simulations ensured optimal surface plasmon excitation, whereas the non-polarized light source used in the microscope contained a mixture of different polarizations and was unable to effectively couple the surface plasmons. A combination of the above mentioned phenomena are responsible for much lower emission intensities than the theoretically possible maximum intensities in the case of these plasmonic nanostructures. Similar observations have been reported by Cui et al [20] for microscopic observation of silver gratings coated with Cy5 dye, showing an optimum enhancement factor of 30 times when compared to a glass slide using an epi-fluorescence optical setup. It should also be noted that the fluorescence response on the flat silver film was about three times greater than on glass slides, which is greater than the mirror effect (two times enhancement). This 
shows that the enhancement of plasmonic excitation also occurs to some extent on a flat metal film due to surface roughness.

\subsubsection{Conclusion}

In conclusion, we have demonstrated extraordinary fluorescence enhancement from nano-gap embedded plasmonic substrates fabricated using an HD-DVD as a starting mold. The extreme field concentration in this case occurs at the nanoscale due to the interaction between localized and propagating surface plasmons. The nano-gap regions embedded in the gratings show much higher field enhancement than nano-gaps on a flat surface, thereby demonstrating the importance of these nanostructures. Theoretical simulations also reveal that the TM modes for these structures exhibit very high electromagnetic field intensities, up to a factor of almost 300. The fluorescence enhancement from these nanostructures was experimentally observed using a broadband light source, showing maximum enhancement of 118 times when compared to a microscope glass slide.

\subsubsection{Supplimentary information}

\section{Fluorescence Image Analysis}

Fluorescence studies of the samples were carried out using an Olympus BX51WI Epi-fluorescence Microscope with a $300 \mathrm{~W}$ Xenon white light source using a FITC filter and a 10x objective. A highresolution CMOS camera (Orca Flash 2.8, Hamamatsu Photonics, and InternalGain-200) was used to obtain the fluorescence micrographs with different exposure times. To reduce the intensity of the incident light on the samples to prevent photo bleaching, a neutral density filters-U-25ND6 (6\% transmission) was used. Fluorescence image analysis was done using open source software ImageJ; to measure the average intensity from all the images obtained using the in-built function in the software. The intensity of the nanogaps was measured by using the "adjust threshold" function and analyzing just the nano-gaps to measure the intensity. Then, the "analyze particles" in-built function was used to form an outline of the nano-gaps and separate them from the background grating as shown in Figure S1 below. After separating the nanogaps from the grating region, the mean and maximum values of the pixel intensities in each enclosed region were obtained which were used to calculate the mean enhancement factors and maximum enhancement factors, respectively. The mean enhancement factors were also calculated for different exposure times of the CMOS detector for all the various substrates-flat silver film, flat silver film with nano-gaps, silver 
gratings, glass, and silver gratings with nano-gaps. An optimum exposure time of $40 \mathrm{~ms}$ was found as shown Table 1. The background values correspond to the fluorescence intensities measured without any layer of Rhodamine-doped PMSSQ and the enhancement factors were calculated using equation 3.

\begin{tabular}{|l|l|l|l|l|l|l|}
\hline & $\begin{array}{l}\text { Nano-gaps } \\
\text { only }\end{array}$ & $\begin{array}{l}\text { Nanno-gaps } \\
\text { Gratings }\end{array}$ & Flat Ag & $\begin{array}{l}\mathrm{Ag} \\
\text { gratings }\end{array}$ & glass & $\begin{array}{l}\text { Background- } \\
\text { glass }\end{array}$ \\
\hline $40 \mathrm{~ms}$ & 137 & 936 & 86 & 380 & 65 & 52 \\
\hline $60 \mathrm{~ms}$ & 173 & 1287 & 107 & 544 & 74 & 54 \\
\hline $80 \mathrm{~ms}$ & 210 & 1795 & 128 & 706 & 84 & 55 \\
\hline $100 \mathrm{~ms}$ & 258 & 2171 & 148 & 870 & 93 & 57 \\
\hline & EF (gratings) & EF (NG gratings) & $\begin{array}{l}\text { EF } \\
\text { (ng- } \\
\text { flat) }\end{array}$ & $\begin{array}{l}\text { EF(flat } \\
\mathrm{Ag})\end{array}$ & $\begin{array}{l}\text { background } \\
\text {-flatAG }\end{array}$ & $\begin{array}{l}\text { background - Ag } \\
\text { grating }\end{array}$ \\
\hline $40 \mathrm{~ms}$ & 25 & 68 & 7 & 3 & 52 & 53 \\
\hline $60 \mathrm{~ms}$ & 24 & 62 & 6 & 3 & 54 & 55 \\
\hline $80 \mathrm{~ms}$ & 22 & 60 & 5 & 2 & 56 & 57 \\
\hline $100 \mathrm{~ms}$ & 23 & 59 & 6 & 3 & 58 & 60 \\
\hline
\end{tabular}

\subsubsection{Table 2 Enhancement factors and average intensity values}

Table 1. Enhancement factors and average intensity values for the different substrates for different exposure times of the CMOS camera.

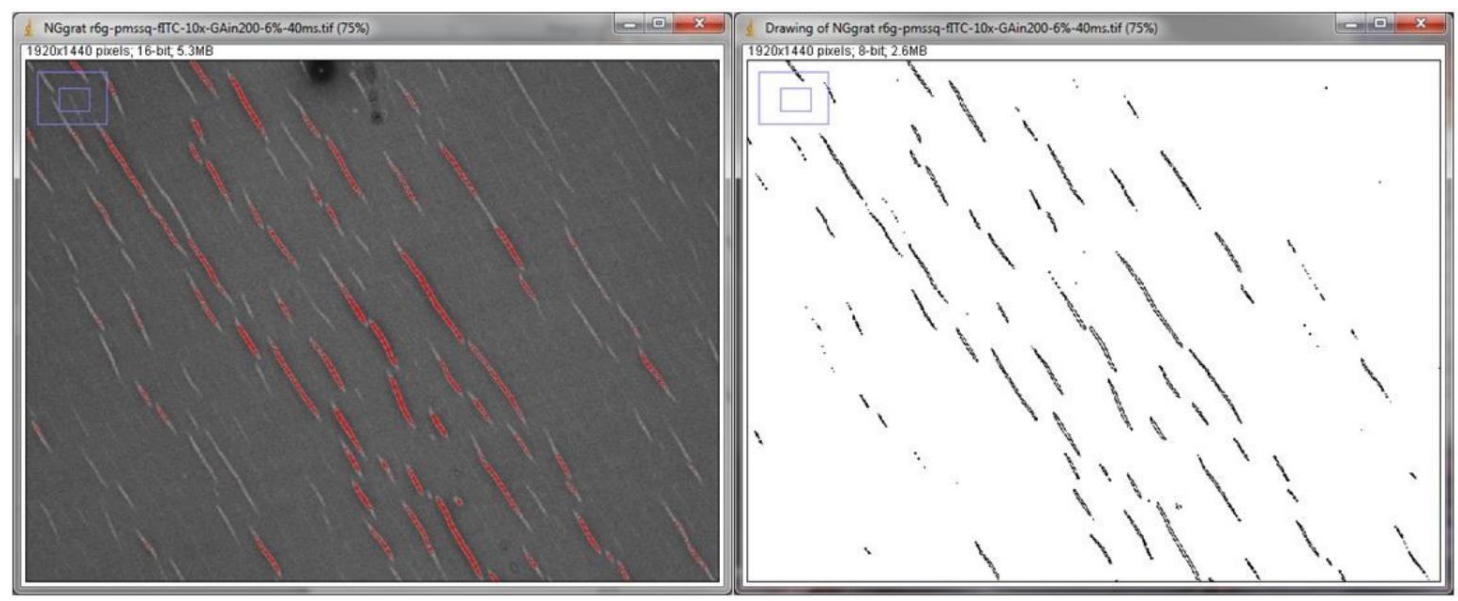

\subsubsection{Figure 19 ImageJ analysis}

Figure S1. The two images above show the ImageJ analysis windows displaying how the nano-gaps are separated from the background grating region in the image based on a threshold intensity.

\section{FDTD Simulation Details}

FullWAVE from RSOFT, Inc. was used to simulate the electric field distribution for the silver nanostructures using the finite different time domain (FDTD) method. The reflectance spectra (Figure S2) for the silver gratings were obtained for transverse magnetic (TM) -polarized $495 \mathrm{~nm}$ light with varying 
number of grating periods, $\mathrm{Np}$, using perfectly matched layer (PML) boundary conditions. It is seen as Np is increased from 1 to 29 , the SPR angle denoted by the sharp dip in reflectivity approaches the surface plasmon resonance $(\mathrm{SPR})$ angle for a periodic boundary condition where $\mathrm{Np}=\infty$. Therefore, for calculating the electric field distributions, a 21 period grating was chosen to save computational time and memory.

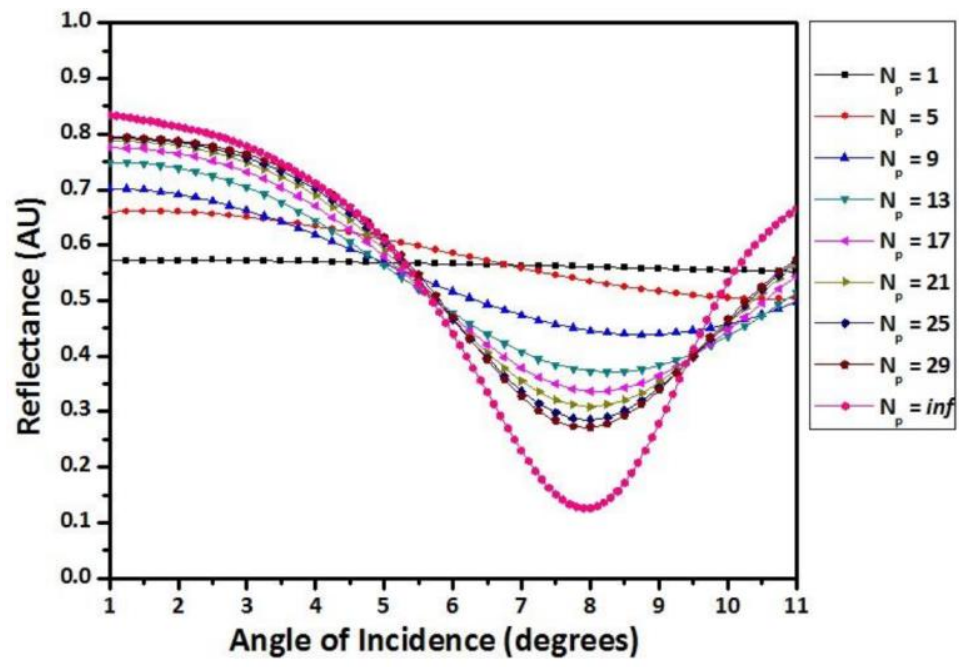

\subsubsection{Figure 20 Reflectance spectra for silver gratings}

Figure S2. Reflectance spectra obtained for silver gratings with a grating pitch, $\Lambda=395 \mathrm{~nm}$ and a grating height of, $\mathrm{h}=60 \mathrm{~nm}$ with different number of periods, $\mathrm{Np}$, with $\mathrm{Np}=\inf$ denoting a periodic boundary condition.

\subsubsection{Acknowledgments}

The authors would like to acknowledge support from the National Science Foundation grant number 1102070 and the Center for Micro/Nano Systems and Nanotechnology at the University of Missouri, Columbia.

\subsubsection{Contributions}

Drew Menke was responsible for the investigation of the surface properties of the plasmonic gratings using the Cornish labs homebuilt objective and prism based TIRF microscope.

\subsubsection{References}

1. Gramotnev D K and Bozhevolnyi S I 2010 Plasmonics beyond the diffraction limit Nature Photon. $483-91$ 
2. Ozbay E 2006 Plasmonics: merging photonics and electronics at nanoscale dimensions Science 311 189-93

3. Schuller J A, Barnard E S, Cai W, Jun Y C, White J S and Brongersma M L 2010 Plasmonics for extreme light concentration and manipulation Nature Mater. 9 193-204

4. Zhu X, Zhang Y, Zhang J, Xu J, Ma Y, Li Z and Yu D 2010Ultrafine and smooth full metal nanostructures for plasmonics Adv. Mater. 22 4345-9

5. Stockman M I 2011 Nanoplasmonics: the physics behind the applications Physics Today AIP 64 $39-44$

6. Hill M T 2009 Nanophotonics: lasers go beyond diffraction limit Nature Nanotechnol.4 706-7

7. Baker G A and Moore D S 2005 Progress in plasmonic engineering of surface-enhanced Ramanscattering substrates toward ultra-trace analysis Anal. Bioanal. Chem. 382 1751-70

8. Stranik O, McEvoy H M, McDonagh C and MacCraith B D 2005 Plasmonic enhancement of fluorescence for sensor applications Sensors Actuators B 107 148-53

9. MacDonald K F, Samson Z L, Stockman M I and Zheludev N I 2009 Ultrafast active plasmonics Nature Photon. $355-8$

10. Weissleder R 2006 Molecular imaging in cancer Science 312 1168-71

11. Achilefu S 2007 Biophotonics: unravelling animal anatomy Nature Photon. $1496-7$

12. Lakowicz J R and Masters B R 2008 Principles of fluorescence spectroscopy, Third Edition J. Biomed. Opt. 13029901

13. Wijaya E, Lenaerts C, Maricot S, Hastanin J, Habraken S, Vilcot J-P, Boukherroub R and Szunerits S 2011 Surface plasmon resonance-based biosensors: from the development of different SPR structures to novel surface functionalization strategies Curr. Opin. Solid State Mater.Sci.15208-24

14. Yu F, Yao D and Knoll W 2003 Surface plasmon field-enhanced fluorescence spectroscopy studies of the interaction between an antibody and its surface-coupled antigen Anal. Chem. 75 $2610-7$

15. Lakowicz J 2006 Plasmonics in biology and plasmon-controlled fluorescence Plasmonics 1 5-33 
16. Kinkhabwala A, Yu Z, Fan S, Avlasevich Y, Mullen K and Moerner W E 2009 Large singlemolecule fluorescence enhancements produced by a bowtie nanoantenna Nature Photon. 3 654-7

17. Kretschmann E 1968 Radiative decay of non-radiative surface plasmons excited by light Z. Naturf. A $232135-6$

18. Otto A 1968 Excitation of nonradiatve surface plasma waves in silver by the method of frustrated total reflection Z. Phys. 216 398-410

19. Raether H 1988 Surface Plasmons on Smooth and Rough Surfaces and on Gratings (Berlin: Springer)

20. Cui X, Tawa K, Hori H and Nishii J 2010 Tailored plasmonic gratings for enhanced fluorescence detection and microscopic imaging Adv. Funct. Mater. 20 546-53

21. Chandezon J, Dupuis M T, Cornet G and Maystre D 1982Multicoated gratings: a differential formalism applicable inthe entire optical regionJ. Opt. Soc. Am. 72 839-46

22. Cui X, Tawa K, Kintaka K and Nishii J 2010 Enhanced fluorescence microscopic imaging by plasmonic nanostructures: from a 1D grating to a 2D nanohole array Adv. Funct. Mater. 20 94550

23. Yokota Y, Ueno K and Misawa H 2011 Essential nanogap effects on surface-enhanced Raman scattering signals from closely spaced gold nanoparticles Chem. Commun. 47 3505-7

24. Willets K A and Van Duyne R P 2007 Localized surface plasmon resonance spectroscopy and sensing Annu. Rev. Phys. Chem. 58 267-97

25. Capasso F, Genevet P, Tetienne J P, Gatzogiannis E, Blanchard R, Kats M A and Scully M O 2011 Coupled nanocavity-grating resonances: large plasmonic enhancement of nonlinear optical phenomena CLEO: Conf. on Lasers and Electro-Optics 2011 pp 1-2

26. Genevet P, Tetienne J-P, Blanchard R, Kats M A, Muller J P B, Scully M O and Capasso F 2011 Enhancement of optical processes in coupled plasmonic nanocavities [Invited] Appl. Opt. 50 G56-62

27. Genevet P, Tetienne J-P, Gatzogiannis E, Blanchard R,Kats M A, Scully M O and Capasso F 2010 Large enhancement of nonlinear optical phenomena by plasmonic nanocavity gratings Nano Lett. $104880-3$ 
28. Miyazaki H T and Kurokawa Y 2006 Controlled plasmon resonance in closed metal/insulator/metal nanocavities Appl. Phys. Lett. 89211126

29. Søndergaard T and Bozhevolnyi S I 2007 Metal nano-strip optical resonators Opt. Express 15 4198-204

30. Renger J, Quidant R, van Hulst N and Novotny L 2010 Surface-enhanced nonlinear four-wave mixing Phys. Rev. Lett. 104046803

31. Coyle S, Netti M C, Baumberg J J, Ghanem M A, Birkin P R, Bartlett P N and Whittaker D M 2001 Confined plasmons in metallic nanocavities Phys. Rev. Lett. 87176801

32. Hatab N A, Hsueh C-H, Gaddis A L, Retterer S T, Li J-H, Eres G, Zhang Z and Gu B 2010 Freestanding optical gold bowtie nanoantenna with variable gap size for enhanced Raman spectroscopy Nano Lett. 10 4952-5

33. Schuck P J, Fromm D P, Sundaramurthy A, Kino G S and Moerner W E 2005 Improving the mismatch between light and nanoscale objects with gold bowtie nanoantennas Phys. Rev. Lett. 94 017402

34. Chou S Y, Krauss P R, Zhang W, Guo L and Zhuang L 1997 Sub-10 nm imprint lithography and applications J. Vac. Sci. Technol. B 15 2897-904

35. Deng X, Braun G B, Liu S, Sciortino P F, Koefer B, Tombler T and Moskovits M 2010 Singleorder, subwavelength resonant nanograting as a uniformly hot substrate for surface-enhanced Raman spectroscopy Nano Lett. 10 1780-6

36. Huebner U, Weber K, Cialla D, Schneidewind H, Zeisberger M, Meyer H G and Popp J 2011 Fabrication and characterization of silver deposited micro fabricated quartz arrays for surface enhanced Raman spectroscopy (SERS) Microelectron. Eng. 88 1761-3

37. Singh B K and Hillier A C 2006 Surface plasmon resonance imaging of biomolecular interactions on a grating-based sensor array Anal. Chem. 78 2009-18

38. Fontana E 2004 Theoretical and experimental study of the surface plasmon resonance effect on a recordable compact disk Appl. Opt. 43 79-87

39. Kaplan B, Guner H, Senlik O, Gurel K, Bayindir M and Dana A 2009 Tuning optical discs for plasmonic applications Plasmonics 4 237-43 
40. Sedoglavich N, Kunnemeyer R, Talele S R and Sharpe J C 2008 Phase-polarisation contrast for surface plasmon resonance based on low cost grating substrates Curr. Appl. Phys. 8 351-4

41. Gurel K 2009 Resonant transmission of light through surface plasmon structures Appl. Phys. Lett. 94233102

42. Wilbur J L, Kumar A, Kim E and Whitesides G M 1994Microfabrication by microcontact printing ofself-assembled monolayersAdv. Mater.6600-4

43. Perl A, Reinhoudt D N and Huskens J 2009 Microcontact printing: limitations and achievements Adv. Mater. 21 2257-68

44. Lee J, Shim W, Lee E, Noh J-S and Lee W 2011 Highly mobile palladium thin films on an elastomeric substrate: nanogap-based hydrogen gas sensors Angew. Chem. Int. Edn 50 5301-5

45. Xia Z C and Hutchinson J W 2000 Crack patterns in thin films J. Mech. Phys. Solids 48 1107-31

46. Liang J, Huang R, Pŕevost J H and Suo Z 2003 Evolving crack patterns in thin films with the extended finite element method Int. J. Solids Struct. 40 2343-54

47. Ghatak A, Mahadevan L, Chung J Y, Chaudhury M K and Shenoy V 2004 Peeling from a biomimetically patterned thin elastic film Proc. R. Soc. Lond. A 460 2725-35

48. Jiang Y, Wang H-Y, Wang H, Gao B-R, Hao Y-W, Jin Y, Chen Q-D and Sun H-B 2011 Surface plasmon enhanced fluorescence of dye molecules on metal grating films J. Phys. Chem. C 115 $12636-42$

49. Romanato F, Kang H K, Lee K H, Ruffato G, Prasciolu M and Wong C C 2009 Interferential lithography of 1D thin metallic sinusoidal gratings: accurate control of the profilefor azimuthal angular dependent plasmonic effects and applications Microelectron. Eng. 86 573-6

50. Ĺopez-Rios T, Mendoza D, Garćı-Vidal F J, Śanchez-Dehesa J and Pannetier B 1998 Surface shape resonances in lamellar metallic gratings Phys. Rev. Lett. 81 665-8

51. Dror Sarid W C 2010 Modern Introduction to Surface Plasmons (Cambridge: Cambridge University Press)

52. Kane Y 1966 Numerical solution of initial boundary value problems involving Maxwell's equations in isotropic media IEEE Trans. Antennas Propagat. 14 302-7 
53. Taflove A 1995 Computational Electrodynamics: The Finite Difference Time Domain Method (Boston, MA: Artech House Publishers)

54. Oubre C and Nordlander P 2004 Optical properties of metallodielectric nanostructures calculated using the finite difference time domain method J. Phys. Chem. B 108 17740-7

55. Kuhn H 1970 Classical aspects of energy transfer in molecular systems J. Chem. Phys. 53 101-8

56. Tawa K, Hori H, Kintaka K, Kiyosue K, Tatsu Y and Nishii J 2008 Optical microscopic observation of fluorescence enhanced by grating-coupled surface plasmon resonance Opt. Express $169781-90$

57. Thomas K G and Kamat P V 2003 Chromophore-functionalized gold nanoparticles Acc. Chem. Res. 36 888-98

58. Hung Y-J, Smolyaninov I I, Davis C C and Wu H-C 2006 Fluorescence enhancement by surface gratings Opt. Express 14 10825-30

59. Hori H, Tawa K, Kintaka K, Nishii J and Tatsu Y 2009Influence of groove depth and surface profile on fluorescence enhancement by grating-coupled surface plasmon resonance Opt. Rev. 16 $216-21$

60. Hecht E 2002 Optics (San Francisco, CA: Pearson Education)

61. Schwartz J J, Stavrakis S and Quake S R 2010 Colloidal lenses allow high-temperature singlemolecule imaging and improve fluorophore photostability Nature Nanotechnol.5127-32

62. Im H, Bantz K C, Lindquist N C, Haynes C L and Oh S-H 2010 Vertically oriented sub-10 nm plasmonic nanogap arrays Nano Lett. 10 2231-6

63. Lakowicz J R 2005 Radiative decay engineering 5: metal-enhanced fluorescence and plasmon emission Anal. Biochem. 337 171-94

64. Liebermann T and Knoll W 2000 Surface-plasmon field-enhanced fluorescence spectroscopy Colloids Surf. A 171 115-30 


\subsubsection{Chapter 3: Single Molecule Oscillations of an RNA/DNA Duplex in a Plasmonic Nanocavity}

As found in: Sagnik Basuray, Avinash Pathak, Biyan Chen, Drew Menke, Charles M. Darr, Keshab

Gangopadhyay, Peter V. Cornish, Shubhra Gangopadhyay, "Single Molecule Oscillations of an RNA/DNA Duplex in a Plasmonic Nanocavity”, J Nanomed Nanotechnol, 06, 03, 2015.

Copyright: (c) 2015 Basuray S, et al. This is an open-access article distributed under the terms of the Creative Commons Attribution License, which permits unrestricted use, distribution, and reproduction in any medium, provided the original author and source are credited.

\subsubsection{Abstract}

We report the visualization of single molecule dynamics in epifluorescence mode through extraordinary plasmonic enhancement provided by silver grating with embedded nanocavities. Cy3/Cy5labeled DNA/RNA hybrid duplexes were affixed to SiO2-capped silver gratings produced by soft lithography process. Tracking single-molecule fluorescence revealed damped sub-1 Hz periodic Cy3 intensity fluctuations with strong dependence on the bulk $\mathrm{MgCl} 2$ concentration. Extreme concentration of electric field at the nanocavity edge induces plasmonic heating, which sets up convection deep within the nanocavity. Local fluctuations in $\mathrm{Mg} 2+$ ion concentration promote a bent or unbent duplex conformational state, respectively, by varying degrees of negative charge screening along the duplex backbone. These oscillations continue until the duplex conformational state stabilizes or the dyes bleach. This unique molecular behavior in the nanocavity could be used to study duplex complementarity, structural polymorphisms, and protein-nucleic acid interactions at the single molecule level.

\subsubsection{Introduction}

Single-molecule (SM) analytical techniques have proven capable of revealing elusive biomolecular interaction fundamentals critical to advancing our knowledge of complex cellular pathways, mechanisms of disease pathogenesis, and next-generation translational research in novel drug design [1-4]. SM studies monitor the stochastic behavior of individual biomolecules under specific experimental 
conditions (e.g. temperature, salt concentration, enzyme substrates, analytes of interest, etc.) through the lens of a high-fidelity reporter (e.g. fluorescence). Elucidation of SM dynamics by fluorescence requires experimental setups that isolate individual molecules and simultaneously overcome the poor intrinsic signal-to-noise ratio (SNR) of single fluorophore labels over background. Using quartz as the immobilization substrate mitigates glass auto fluorescence, but epifluorescence microscopes introduce additional noise through excitation of the bulk solution. This is most often addressed through the use of complex optical setups such as total internal reflection (TIR) microscopes [5-7]. Alternatively, SM fluorescence and SNR can be improved through resonant photon coupling to surface plasmon polaritons extant at a metal-dielectric interface (SPR) [8]. SPR coupling to plasmonic active metals (e.g. gold, silver) generates a highly-concentrated evanescent electric (E-) field that is typically several-fold higher magnitude than the incident field, significantly increasing excitation rate as well as radiative decay rate of fluorophores immobilized near the plasmonic surface with respect to a quartz surface [9-11].

Matching the optical momentum of incident light to the propagation constant of the surface plasmons is difficult to achieve with flat metal films except through the use of high index prisms, but may be realized in free space by introducing wavelength-scale periodic perturbations on the metal-dielectric interface (i.e. gratings) [12-14]. Plasmonic E-field enhancement can also be produced locally (LSPR) by concentrating coupled light using nanostructured surface morphologies with geometric singularities such as bowties/ nanoantennae, [15,16]. nanolenses, [17] tip resonators, [18] and nanohole arrays [19]. Integrating LSPR light concentration through nanoscale geometric singularities with SPR coupling substrates leads to size-tunable, additive field enhancement and concentration [20-22]. Such enhancement may be sufficient to permit visualization of single molecules using less expensive and complex epifluorescence optical setups.

In this work, we have used a nanoscale periodic metallic grating with embedded nanocavities fabricated by a cost-efficient soft lithography process to achieve fluorescence enhancement factors sufficient to visualize single molecule dynamics in an epifluorescence microscope. The plasmonic substrate takes advantage of the strong free space light coupling properties of silver SPR gratings and field concentration at the discontinuities in the metal provided by the randomly generated 20-200 nm nanocavities, which we have previously shown to provide fluorophore enhancement exceeding 100 -fold with respect to glass [14]. We demonstrate the single molecule fluorescence capabilities of this system 
using a fluorophore-pair-labeled RNA/DNA hybrid duplex. Moreover, we report a unique observation that those duplex molecules identified as residing deep within the nanocavities exhibit ion-concentrationdependent conformational rearrangements in the form of fluorescence intensity oscillations. This behavior is related back to the duplex structure, nanocavity dimension, and significant charge screening set up by the high ionic strength in the nanocavity. We anticipate this observation could be extended to identify polymorphisms or duplex-target interactions through frequency modulation at a specific ion concentration.

\subsubsection{Materials and Methods}

\section{Sample preparation for single molecule experiments}

Silver gratings were fabricated on silicon substrates using a soft lithography stamping process replicating an HD DVD grating mold as described previously [14]. Briefly, poly-(methylsilsesquioxane) (PMSSQ, GR650F, Techneglas) was stamped onto a cleaned silicon wafer followed by sputter deposition of a $2 \mathrm{~nm}$ titanium adhesion layer and $100 \mathrm{~nm}$ silver layer. Mechanical stresses incurred in the stamping process result in formation of randomly sized and distributed nanocavities. A $10 \mathrm{~nm} \mathrm{SiO} 2$ capping layer was then deposited by e-beam physical vapor deposition.

Cy3/Cy5 dye pair-labeled DNA-RNA (duplex) hybrid molecules were immobilized on both SiO2capped silver gratings with embedded nanocavities and unmodified glass coverslips for comparison. A 50 $\mu \mathrm{L}$ aliquot of $1 \times \mathrm{T} 50$ buffer solution (10 mM TRIS, $50 \mathrm{mM} \mathrm{NaCl}, \mathrm{pH} 8.0)$ was first dispensed on the substrate surfaces followed by $10 \mathrm{~min}$ incubation with an additional $50 \mu \mathrm{L}$ of $1 \mu \mathrm{g} / \mu \mathrm{L}$ biotinylated bovine serum albumin (BSA-Biotin) in T50 buffer. Excess BSA-Biotin was removed by rinsing with $50 \mu \mathrm{L}$ fresh T50 buffer. Neutravidin $(50 \mu \mathrm{L})$ was then added and incubated for 5 minutes followed by another T50 buffer rinse. Finally, the duplex molecules were dispensed on the surface in imaging buffer $(20 \mu \mathrm{L} 5 \times$ T50 buffer, $15 \mu \mathrm{L} 2 \mathrm{M} \mathrm{MgCl}, 1.6 \mu \mathrm{L}$ D-Glucose, $1 \mu \mathrm{L}$ Gloxy, and 74.2 $\mu \mathrm{L}$ Trolox) to avoid fluorophore blinking.

SiO2-capped silver grating platforms were cleaned using highpressure nitrogen flow just prior to flow cell fabrication. Solvent-based washing was avoided to prevent damage to the silver or underlying PMSSQ layer. The flow cell was constructed by drilling entry and exit holes through a glass or quartz slide using a Dremel tool with a $0.75 \mathrm{~mm}$ diamond-coated bit. Glass spacers were secured at the edges by 5 minute epoxy (Devcon Home) and the grating was secured between the entry/exit holes. Slides and 
coverslips were cleaned by high-pressure N2 flow, passed over a diffuse flame, and allowed to cool. Double-sided tape was then used to separate each set of holes and define the ends and the cell was capped by a N2-cleaned coverslip. Finally, the ends were closed using a 5-minute epoxy.

\section{Instrumentation and measurement details}

Single molecule fluorescence measurements were performed on an Olympus IX-71 inverted microscope with custom epifluorescence excitation setup. Excitation sources were $100 \mathrm{~mW} 532 \mathrm{~nm}$ or 642 nm diode-pumped solid-state lasers (Spectra Physics, Excelsior One) to monitor Cy3/Cy5 FRET or only Cy5 emission, respectively. Laser power was attenuated to appropriate fluence ( $4.6 \mathrm{~mW}$ ) by a $532 \mathrm{~nm}$ or $633 \mathrm{~nm}$ zero-order half-wave plate (Thor Labs, WPH05M-532/-633), respectively, and neutral density filters (ThorLabs, NE40B, NE30B, NE20B, NE10B, NE06B, NE05B, NE04B, NE03B, and NE02B). Fluorophore emission was collected using either an UPlanSApo 100× oil-immersion objective (Olympus, $\mathrm{NA}=1.40$ ) or UPlanSApo $60 \times$ water-immersion objective (Olympus, $\mathrm{NA}=1.20$ ) using a zt532rdc dichroic and HQ545lp long-pass filter (Chroma) with additional 630dcxr dichroic/shot-pass filter to separate Cy3 and Cy5 emission into individual channels. Fluorescence intensities were recorded on an Andor iXon+ EMCCD camera at a rate of 10 frames per second (fps) for a total length of 1200 frames and $100 \mathrm{~ms}$ integration time per frame. Camera gain and scaling factors were kept constant for all substrates to allow for direct comparison of intensity values, signal-to-noise ratio, and calculation of fluorescence enhancement factors.

\section{Fluorescence recording analysis}

Fluorescence recordings were analyzed by MATLAB ${ }^{\circledR}$ for singlemolecule fluorescence intensity and power spectral density (PSD) of the intensity-time plots. Considering the low (pM) concentration of duplex added to the substrates, molecules are assumed to be sufficiently isolated so that intensity above the background noise represent individual molecules. Pixel intensities above the average first-frame intensity were recorded and analyzed across all frames as potential single-molecule fluorophores while belowaverage values are ignored. Presence of solitary dye-labeled duplex molecules was confirmed by identification of a single-step photobleaching event. FRET efficiency was analyzed by evaluating relative pixel intensity in both the donor and acceptor emission channel. Correct pixel mapping was confirmed daily prior to any experiment by running a plain glass slide with immobilized red fluorescent beads. 
PSD calculation was performed using a Fast Fourier Transform (FFT) on the entire frameset from a fluorescence recording (1200 frames per 2 min recording). The Nyquist criterion [23]. gives a maximum recordable frequency of $5 \mathrm{~Hz}$. Once a FFT command was executed on each trace file, the conjugate of the resultant FFT value was multiplied by the FFT values to derive the ultimate power contained within each frequency. This was then plotted with frequencies from 0 to $5 \mathrm{~Hz}$ along the $\mathrm{x}$-axis. Pre- and postphotobleaching regions were isolated with the post-photobleaching region then representing the background for calculating enhancement and PSD.

\subsubsection{Results}

Plasmonic-enhanced single molecule Förster resonance energy transfer (smFRET) studies were performed using an RNA-DNA hybrid structure hereafter referred to as 'duplex' (Figure 1A) affixed to our $\mathrm{SiO} 2$-capped silver plasmonic gratings with embedded nanocavities (Figure 1B) [14]. The duplex consists of a 137-base RNA backbone with 29-bp biotinylated DNA tethered to the substrate, 11-base open RNA region, 20-bp DNA labeled with Cyanine 3 (Cy3) donor and Cyanine 5 (Cy5) acceptor at opposite ends, and 77-base RNA tail extending into the medium. The $6.8 \mathrm{~nm}$ (20-bp) Cy3-Cy5 dye separation provides only 20\% FRET efficiency given the nominal Förster distance Ro=5.4 nm, [24]. so that the Cy3 donor fluorescence typically dominates under normal circumstances. The conformal $10 \mathrm{~nm} \mathrm{SiO} 2$ acts as a spacer layer to prevent metal-based fluorescence quenching, mitigate silver degradation in the aqueous environment, and provide a hydroxylterminated $(-\mathrm{OH})$ surface to promote adsorption of biotinylated BSA. Biotin-streptavidin linkage immobilizes the duplex to the surface, providing a Cy3-dominated fluorescence intensity map visible in epifluorescence mode (Figure 1C). As can be seen in Figure 1C, there is a distribution of fluorescence intensities across the surface with highest intensity located along the gratingnanocavity interface. These were confirmed to be single molecules by the presence of single-step photobleaching, indicating the intensity distribution was due to some variation in the enhancement of individual fluorophores across the substrate.

The plasmonic nanocavities result from shear stresses incurred in the stamp and polymer ink during fabrication and range in size from 20-200 $\mathrm{nm}$ in width and 20-100 $\mathrm{nm}$ in depth, intersecting the grating in randomly distributed and oriented arrangements [14]. Quantitation of the morphology-mediated plasmonic-enhanced E-field strength is fundamental to understanding the associated fluorescence 
enhancement phenomena. Figure 1D shows an electric field distribution at the cross-section of gratings with a $50 \mathrm{~nm}$ wide and $100 \mathrm{~nm}$ deep vertical nanocavity by finite-difference time-domain (FDTD) simulation (details in Supplementary Section 1 (Figures S1-S6)) [14]. For the simulation, the plasmonic substrate was illuminated using $532 \mathrm{~nm}$ light at the associated resonance angle given by the dispersion relation for $400 \mathrm{~nm}$ grating spacing $\left(\theta \mathrm{SP}=8^{\circ}\right)$. Figure 1D shows E-field enhancement $(\mathrm{Ez} / \mathrm{Ez}, 0)$ of as much as $19 \times$ the incident field strength where the propagating surface plasmon polaritons (SPPs) are concentrated at the upper edge of the grating intersection with the nanocavity. Meanwhile, the waterfilled depths within the nanocavity experience much lower E-fields due to field concentration at the geometrical singularity of the nanocavity edge. E-field magnitude increases with increasing height through the nanocavity edge from $\sim 1 \times$ up to $2.5 \times$ the incident field strength in the area just above the nanocavity as shown by the field intensity profile (Figure 1E) of the middle of the nanocavity (the white dashed line in Figure 1D). Figure 2 shows smFRET time traces extracted from multiple SM fluorescence movies recording intensity dynamics across a range of $\mathrm{MgCl} 2$ concentrations from $10 \mathrm{mM}$ to $1 \mathrm{M}$. Duplex mounted on quartz (Figure 2A) or on plasmonic grating surface away from the nanocavities (Figure 2B) exhibit no anticorrelated FRET behavior or intensity fluctuation observable above the noise prior to single-step photobleaching. Such behavior was expected as the dyes were separated to the lower limit of FRET efficiency. However, analysis of the molecules localized in the nanocavities such as those in Figure $2 \mathrm{C}$ displayed sub- $1 \mathrm{~Hz}$ fluorescence intensity oscillations, especially at extremely high $\mathrm{MgCl} 2$ concentrations (Figures 2D - 2F). Statistical analysis showed that these oscillations were not unique to only a few molecules, but seen in more than $50 \%$ of duplex single molecules immobilized within nanocavities (Supplementary Figure S7). It is important to note that $\mathrm{Cy} 3$ intensity oscillations occurring for dyes in the nanocavities (Figure 2D) were recorded in the same movies as non-fluctuating dyes outside the nanocavities (Figure 2B), which suggests there is a unique property 


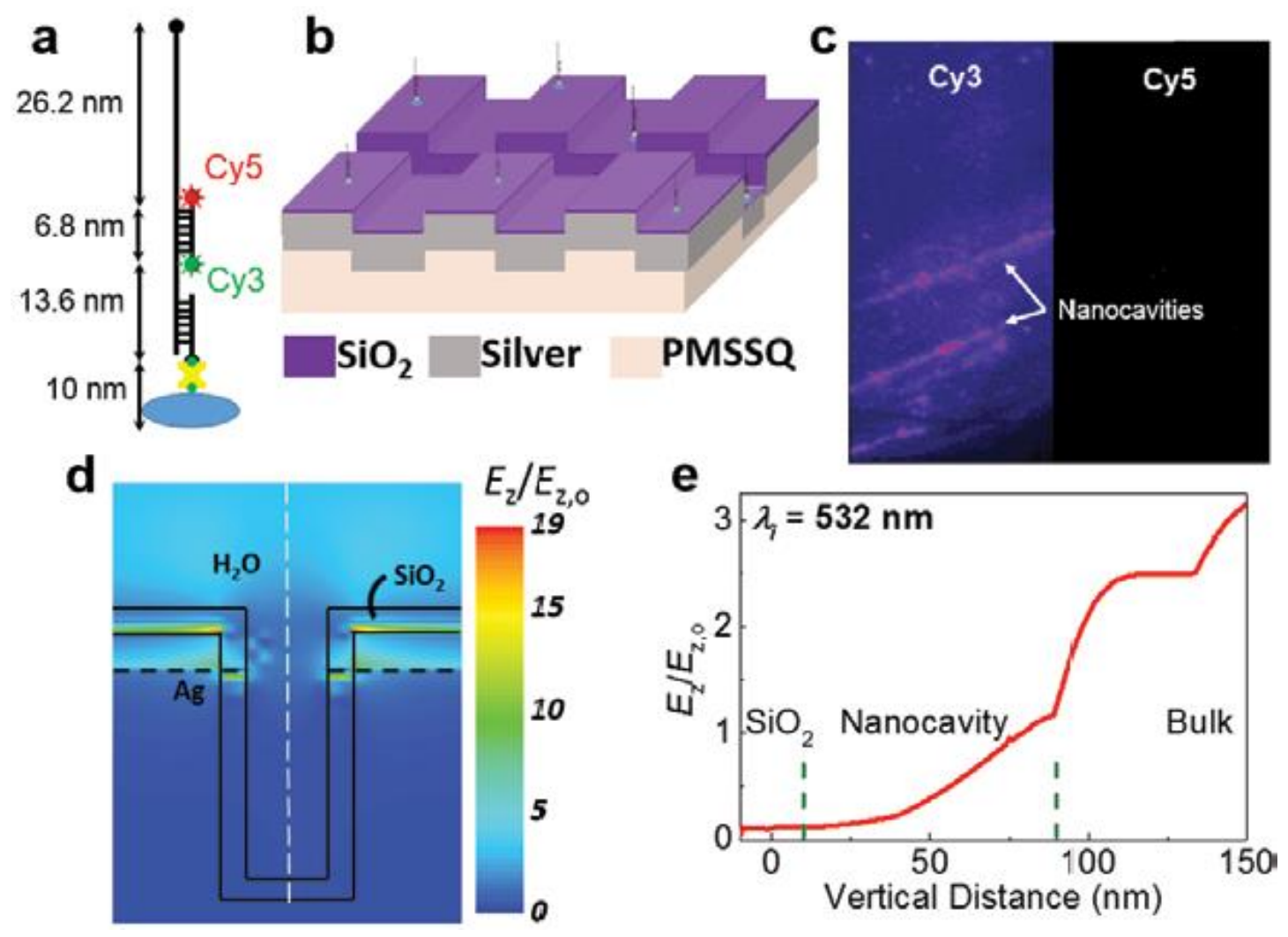

\subsubsection{Figure 21 Experimental design}

Figure 1: (A) Structure of the dye-labeled RNA/DNA duplex; (B) Schematic of the grating with embedded nanocavity; (C) Representative frames from the split-channel Cy3/Cy5 FRET recordings; (D) Simulated electric field distribution at the nanocavity-grating interface (black dashed line indicates the level of the base of the grating); and (E) Electric field depth profile along the middle of the nanocavity (white dashed line in (D))
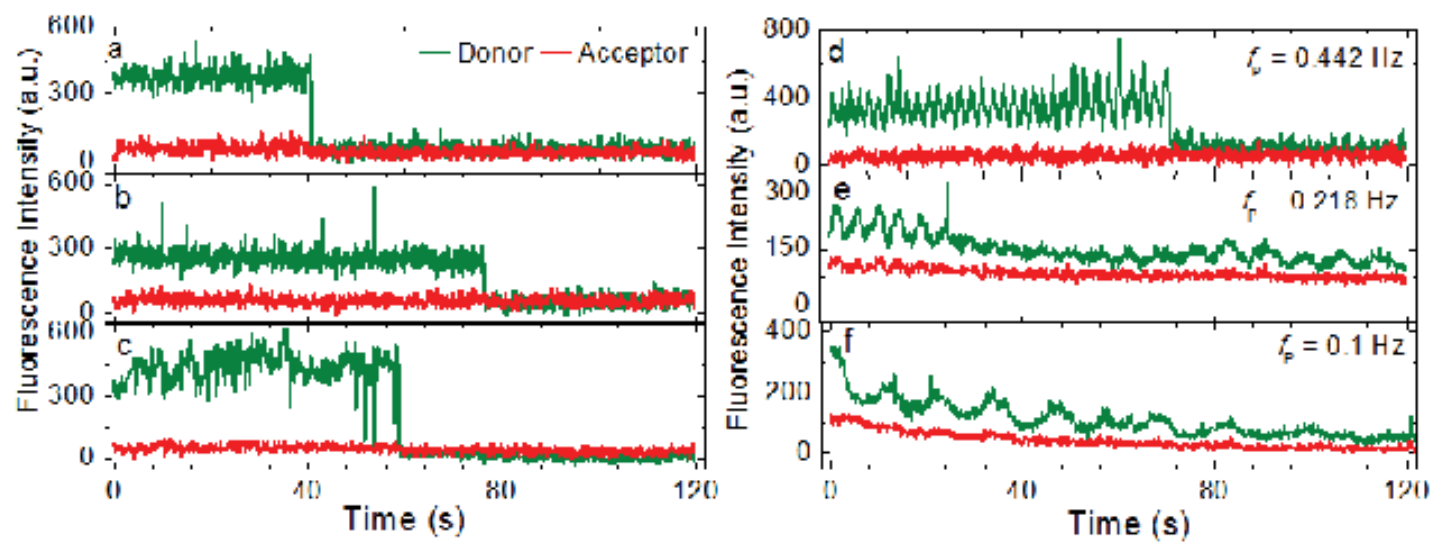

\subsubsection{Figure 22 smFRET traces taken with epi-fluorescence microscope}


Figure 2: (A and B) Cy3/Cy5 smFRET traces measured at $100 \mathrm{mM} \mathrm{MgCl} 2$ for duplex on (A) quartz and (B) the grating surface; (C-F) smFRET traces of duplex localized in the nanocavity measured at (C) 10 mM, (D) $100 \mathrm{mM}$, (E) $300 \mathrm{mM}$, and (F) $1 \mathrm{M} \mathrm{MgCl2}$. The data for quartz substrate was taken with TIRFM, and those for gratings and nanocavities were taken with epi-fluorescence microscope.

of the nanocavities giving rise to this phenomenon. There is also a notable lack of anticorrelated Cy5 intensity fluctuation in those traces showing oscillatory behavior. This will be addressed in the discussion section below.

Power spectral density (PSD) analysis was performed on the smFRET traces exhibiting fluorescence intensity oscillations to identify the characteristic frequencies for each $\mathrm{MgCl} 2$ concentration (Figure 3A). After removal of external noise sources (i.e. Brownian, instrument dark current, etc.), PSD analysis showed that oscillation frequency decreased with increasing $[\mathrm{MgCl} 2]$. from $0.4 \mathrm{~Hz}$ at $100 \mathrm{mM}$ $\mathrm{MgCl} 2$ to $0.1 \mathrm{~Hz}$ at $1 \mathrm{M} \mathrm{MgCl} 2$. At $10 \mathrm{mM} \mathrm{MgCl}$, there were no apparent intensity oscillations observed (Figure 2C). By extrapolating from the frequencies at higher [ $\mathrm{MgCl} 2]$, we find that the most likely frequency for $10 \mathrm{mM} \mathrm{MgCl} 2$ would be obscured by the instrument dark current noise $(\sim 1 \mathrm{~Hz})$ found by PSD analysis of the instrument response postphotobleaching (Supplementary Figure S8).

Fluorescence intensity oscillations report underlying physical phenomena, the identity of which relies on the physical and chemical conditions near the duplex molecule. We know that duplex molecules exhibiting oscillatory behavior are found only at points identified as being part of a nanocavity (Figure 1C). Possible explanations for duplex oscillation in the nanocavities in our case include quenching effects by close proximity to silver, E-field variation resulting in higher localized plasmonic enhancement of Cy3, changes in laser power, and conformational changes in duplex resulting in higher FRET efficiency. The laser power was held constant across all samples at $4.6 \mathrm{~mW}$ and so is not responsible for intensity changes between different samples. Silver quenching effects on $\mathrm{Cy} 3 / \mathrm{Cy} 5$ have been largely negated by inclusion of the $10 \mathrm{~nm} \mathrm{SiO} 2$ spacer layer, which insures the dyes are at least beyond the critical quenching distance for silver $(\sim 8 \mathrm{~nm})$ [14]. Variable plasmonic enhancement would rely on a sharp E-field gradient in the vicinity of the Cy3 label. Such gradients are located at the grating-nanocavity interface where the E-field magnitude is highest (Figure 1D). However, duplex molecules localized at the edges of the nanocavities 
exhibiting highest intensity and, therefore, experiencing highest local E-field displayed no observable periodic oscillations (Supplementary Section 2 and 4). Exclusion of these physical principles as sources of oscillatory behavior leaves conformational changes in the duplex, namely, bending and unbending behavior resulting in increased FRET efficiency between Cy3 and Cy5.

The lack of anticorrelated Cy5 intensity indicative of FRET is attributed to a combination of low FRET efficiency resulting from the dye separation and directional emission of Cy5 wavelengths away from the detector by surface plasmon-coupled emission (SPCE) [10,25-27]. SPCE occurs when the emitted wavelengths and angular conditions satisfy the dispersion relations for plasmonic gratings in a particular environment, converting normally isotropic emission into directional emission in a narrow angular distribution. The CCD camera window has a $5.1^{\circ} \times 10.2^{\circ}$ acceptance cone in these experiments (Figure S4) [25]. As a result, any fluorescence emission occurring at angles larger than the objective capture angle will be lost. SPCE significantly reduced emitted photon capture by the detector of Cy5 emission wavelengths. This reduced capture efficiency is illustrated by the study of Holliday Junction molecules (two adjacent arms are labeled with Cy3 and Cy5 molecules while a third arm is tethered to the surface) on two different substrates: quartz substrate (using TIRF), which does not have an angle-dependent emission profile, and silver gratings with embedded nanocavities, which have highly angle-dependent emission and excitation properties (Figure S5). Anticorrelated behavior is apparent for the molecules on quartz, with the on and off states averaging 0.7 and 0.3 FRET efficiency, respectively. The Cy3 channel intensity is $8-10 \times$ higher on gratings in epifluorescence mode than the molecules on quartz in TIR mode. Meanwhile, the acceptor emission capture is shown to be severely compromised, corresponding to FRET efficiencies in the on and off states of 0.28 and 0.08 , respectively. Assuming that the molecules behave similarly on the two platforms, the energy lost by moving from isotropic (quartz, TIR) to directed (gratings, SPCE) emission can be calculated. The change in FRET on and off states represents a loss of $60 \%$ to $75 \%$ of acceptor emission capture.

Periodic DNA motion has been observed in the presence of external forces such as a secondary laser, $\mathrm{pH}$ modulation, and chemical changes [28-30]. Based on the above observations, the origin, amplitude, and frequency of the oscillatory bending behavior must be strongly dependent on [ $\mathrm{MgCl} 2]$. while independent of silver proximity and variation in E-field magnitude. We further posit that the 
molecules displaying oscillatory behavior should be located deep within the nanocavity. This is supported by the fact that molecules residing on the gratings display no oscillatory behavior (Figure 2B) in the same sample in which nanocavity-bound molecules do oscillate (Figure 2D). Furthermore, the E-field magnitude deep within the nanocavity is much lower than that at the edges and varies by less than $10 \%$ of the incident field strength (Figure 1E), which is consistent with our observation that those molecules at the edges of the nanocavities display no oscillatory behavior.

\subsubsection{Discussion}

The bending of DNA strands shorter than the persistence length ( $<50 \mathrm{~nm}$ or $<150 \mathrm{bp}$ ) [31] is retarded in ambient conditions due to high entropy costs, though there have been observations to the contrary [32,33]. Further, it is well established that high [Mg2+]. significantly reduces the persistence length due to the screening of electrostatic repulsion between the negatively charged phosphate groups on the DNA backbone [34]. The increased charge screening effect with increasing [Mg2+]., leads to more stable collapsed conformational state of the DNA as is illustrated on the right in Figure 4B. The collapsed conformation pulls the dye pair closer together, which could be viewed in our FRET system as a generally lower initial Cy3 intensity at higher salt concentrations (Figure 3B), the Cy5 emission lost outside the viewable range of the camera due to SPCE as explained above. The collapsing effect of increased salt concentration can also be seen in the overall decay in fluorescence intensity at $300 \mathrm{mM}$ and $1 \mathrm{M} \mathrm{MgCl} 2$ (Figures 2E and 2F). This decay is inconsistent with the single-step photobleaching observed in Figures 2A-2D. Instead, the higher salt concentration perturbs the duplex molecules deep within the nanocavities, inducing the cyclical bending/unbending behavior as in Figure 2D, but ultimately trends toward a more stable bent or collapsed duplex conformation. 

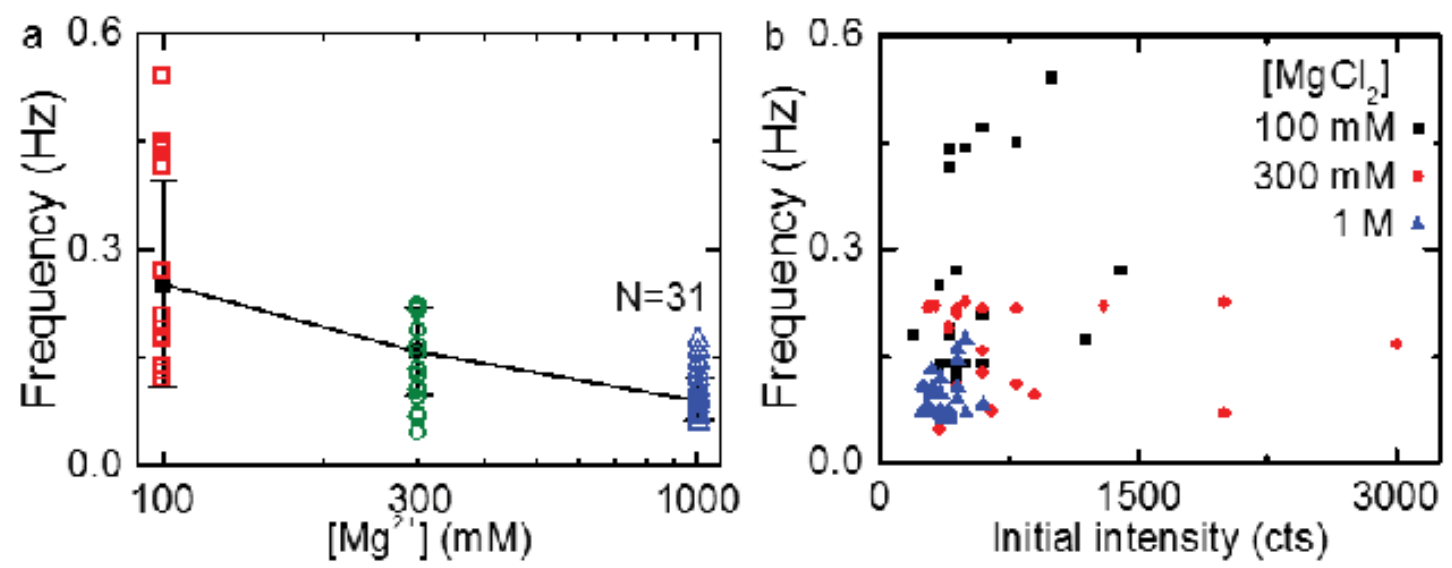

\subsubsection{Figure 23 Fluorophore intensity oscillation frequency}

Figure 3: (A) Fluorophore intensity oscillation frequency as a function of $\mathrm{Mg} 2+$ ion concentration (open points represent frequencies extracted from individual recordings) and (B) Oscillation frequency as a function of $\mathrm{MgCl} 2$ concentration and initial fluorophore intensity.

a

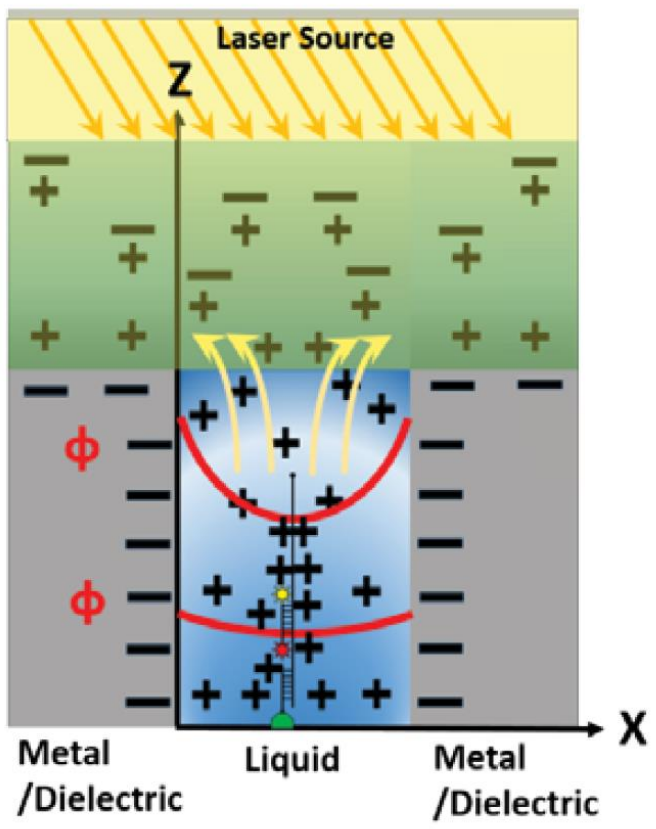

b

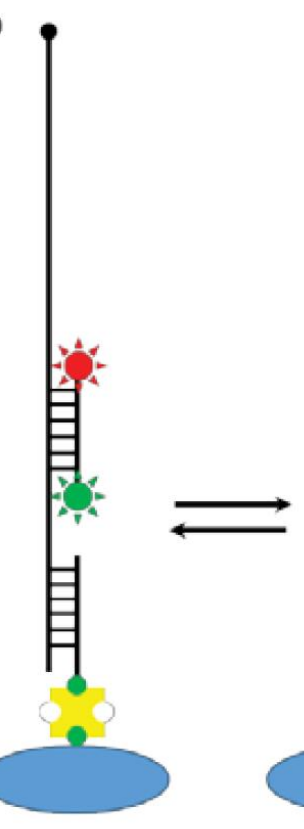

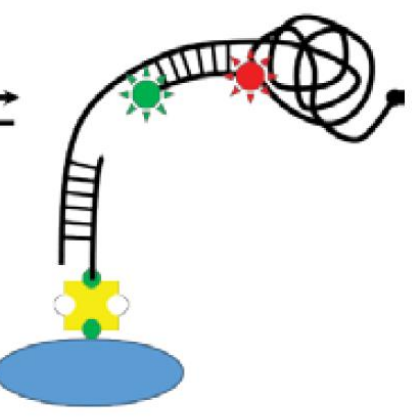

\subsubsection{Figure 24 Schematic of the ion concentration distribution and duplex behavior}

Figure 4: (A) Schematic of the ion concentration distribution within the nanocavity against that in the bulk and the convection induced by the laser source and (B) Bending behavior of duplex in the presence of variable ion flux. 
A similar charge screening effect occurs within the negatively charged SiO2-coated nanocavity; the counterion in the nanocavity should form a double layer with length $\lambda$ on each wall. Using the classical relation equation, $\lambda$ is found to be $\sim 5 \mathrm{~nm}$ for the lowest ion concentration $(10 \mathrm{mM})$. As the channel dimension (h) approaches $h \sim \lambda$, as in a nanocavity, the double layers start to overlap resulting in ionselective membranes [35]. However, ion selectivity in nanocavities can occur even for $h>2 \lambda[36]$. An overlapped double layer effect can be seen here, even though the nanocavity dimensions ( $\mathrm{w}=20-200 \mathrm{~nm}$, $\mathrm{d}=20-100 \mathrm{~nm}$ ) are much larger than the predicted double layer length. If the negatively charged duplex was located deep within the negatively charged $\mathrm{SiO} 2$ nanocavity, it will lead to the formation of a roughly unipolar solution of $\mathrm{Mg} 2+$ and other positive ions while negatively charged ions are simultaneously expelled (Figure 4A), an effect similar to ion-selective nanopores [36].

To determine the physics behind the initiation and continuation of the nanocavity based duplex fluctuations, it is necessary to examine the convective and diffusive mechanisms in the nanochannel and examine the possibility of plasmonic heating causing the oscillations. For plasmonic heating, an external Efield produces localized heating when it undergoes focusing in nanocavities, setting up a convective electroosmotic flux known as the thermal end effect [37]. This thermally-induced ion flux can create convective fluxes leading to disturbances in the concentration profile proportional to the charge inside the nanocavity [38]. However, here as the plasmonic enhanced field focusing is limited to the upper rim of the nanocavity (Figure 1D), thus any associated plasmonic heating will be limited to the surface only. Further, it is well established that diffusive forces dominate over convective forces in plasmonic systems, as illustrated by the associated small dimensionless thermal Péclet number (Pe) of $\sim 10-9$ when calculated using any standard temperature-based method (details in Supplementary Section SI-6). [38]. Thus, there is negligible increase in temperature near the duplex due to plasmonic heating, failing to melt or denature the duplex deep within the nanocavity. This arrangement is confirmed by the presence of fluorescence from molecules in the nanocavity, which would be rapidly removed if plasmonic heating above the melting temperature ( $\sim 65^{\circ} \mathrm{C}$ for the 20-bp duplex region) [39]. occurred near the duplex itself. Further, the diffusive forces relax at the order of $\mu \mathrm{s}$ and, thus, will not contribute to the frequencies observed here. 
However, exchanging the thermal velocity flow with an equivalent electroosmotic flow (due to free charges), the field gradient (osmotic pressure) induced convective flow due to plasmonic E-field focusing can be accounted for using an ionic Peclet number. This ionic Pe $(2.95 \times 10-3)$ is relatively higher (details in Supplementary Section SI-6), which indicates increased convective forces, specifically near the rim of the nanocavity where the field focusing is highest (Figure 1D). Thus, we identify the source of the oscillations as an axial ionic gradient flux resulting in a curved ion concentration profile from the increased convective forces similar to that observed in the thermal end effects from external E-field focusing in nanocavities [37]. The ion concentration profile can propagate into the nanocavity, leading to a subsequent Maxwell stress profile near the DNA duplex and providing sufficient osmotic forces to bend the duplex and begin the oscillatory cycle.

We can now assert that the initial oscillation frequencies are determined by the $[\mathrm{Mg} 2+]$. and localization of the duplex deep within a nanocavity to form an overlapped double layer and have sufficient E-field magnitude to induce thermally stimulated electroosmotic flow. Due to size and depth variations in the randomly generated nanocavities in this experiment, there is an equally wide variety of E-field magnitudes as well as double layer lengths within the nanocavities, leading to high variation in backbone flexibility, initial intensity, and oscillation frequency in the presence of moderately high bulk $[\mathrm{Mg} 2+]$ (Figures 2 and 3B). Meanwhile, the $1 \mathrm{M} \mathrm{MgCl} 2$ results in sufficient high $[\mathrm{Mg} 2+]$ concentration to induce stable collapsed conformational state of the duplex regardless of location, which is evidenced by the lower initial fluorescence intensity and oscillation frequency (Figure 3). This is also illustrated through linking the donor intensity with the salt concentration, which shows wide variation due to the random nanocavity dimensions, but overall lower oscillation frequency and frequency variability with increasing salt concentration (Figure 3B). In the presence of high salt concentration, the molecule oscillates, but the oscillation amplitude damps with time in a system resembling an underdamped mass-spring-dashpot system (Figures 2D and 2E). In this case, the DNA is the mass, the electroosmotic flow and [Mg2+]. gradient is the spring, and stabilization by charge screening is the dashpot or damper. This explains the oscillation of the duplex, for the charge screening effect at high $[\mathrm{Mg} 2+]$. is reversible and depends on system entropy [34]. The end convective flux of $\mathrm{Mg} 2+$ will lead to lowering of the positive ions in the cavity leading to duplex straightening [34]. After duplex straightening, there will be excess surface charge 
inside due to duplex charged backbone. To satisfy this changed electro-neutrality condition inside the nanocavity, it will lead to excess $\mathrm{Mg} 2+$ ions inside the cavity. These excess positive ions should leading to DNA bending. Thus, the energy necessary to reverse the bending process is supplied by the laser fluence and plasmonic field focusing effect [29]. A more quantitative explanation of these phenomena will require detailed simulation and experimentation with focus on characterizing the nanocavity dimension and lengthheight aspect ratio to optimize plasmonic field focusing and, thus, concentration gradient.

In summary, we have detailed a preliminary observation of unique oscillatory DNA/RNA duplex bending behavior in a single molecule fluorescence study using an epifluorescence mode microscope setup. The oscillation frequency was found to be governed by the bulk cation concentration, location of the duplex within the nanocavity, and the duplex molecular composition (Figure S9). This study sets the framework for further analysis of complex molecular interactions with the local environment. Characterizing oscillation frequency, amplitude, and damping rate with respect to varying duplex length, nucleotide content, and base-pair matching could provide abundant information for a library of hybrid duplex structures. Monitoring subsequent changes in a given duplex oscillation amplitude and frequency on addition of a ligand or analyte of interest allows tracking of DNA-ligand interactions as well as single nucleotide polymorphisms, leading to a better understanding of those systems and environmental effects on their interactions. Follow-up studies will be performed with greater focus on molecule- and ionconcentration-specific oscillation frequency and damping rates as well as the influence of the nanocavity dimensions on the extent of this behavior.

\subsubsection{Acknowledgements}

Funding for this work was provided by a grant from the National Science Foundation (Award No. ECCS1102070).

\subsubsection{Contributions}

Drew Menke was responsible for the investigation of the plasmonic gratings with the RNA-DNA hybrid biomolecules using the Cornish labs homebuilt objective and prism based TIRF microscope. Observations of the oscillation found within the data were first reported on by the Menke, Drew. 


\subsubsection{References}

1. Levene MJ, Korlach J, Turner SW, Foquet M, Craighead HG, et al. (2003) Zeromode waveguides for single-molecule analysis at high concentrations. Science 299: 682-686.

2. Armani AM, Kulkarni RP, Fraser SE, Flagan RC, Vahala KJ (2007) Label-free, single-molecule detection with optical microcavities. Science 317: 783-787.

3. Roy R, Hohng S, Ha T (2008) A practical guide to single-molecule FRET. Nat Methods 5: 507-516.

4. Jain A, Liu R, Ramani B, Arauz E, Ishitsuka Y, et al. (2011) Probing cellular protein complexes using single-molecule pull-down. Nature 473: 484-488.

5. Chu S (1991) Laser manipulation of atoms and particles. Science 253: 861-866.

6. Deniz AA, Dahan M, Grunwell JR, Ha T, Faulhaber AE, et al. (1999) Singlepair fluorescence resonance energy transfer on freely diffusing molecules: observation of Forster distance dependence and subpopulations. Proc Natl Acad Sci 96: 3670-3675.

7. Haustein E, Schwille P (2003) Ultrasensitive investigations of biological systems by fluorescence correlation spectroscopy. Methods 29: 153-166.

8. Lakowicz JR (2006) Plasmonics in Biology and Plasmon-Controlled Fluorescence. Plasmonics 1: 5-33.

9. Lakowicz JR (2001) Radiative decay engineering: biophysical and biomedical applications. Anal Biochem 298: 1-24.

10. Lakowicz JR (2005) Radiative decay engineering 5: metal-enhanced fluorescence and plasmon emission. Anal Biochem 337: 171-194.

11. Gramotnev DK, Bozhevolnyi SI (2010) Plasmonics beyond the diffraction limit. Nat Photon 4: 83-89.

12. Homola J, Yee SS, Gauglitz G (1999) Surface plasmon resonance sensors: review. Sensors and Actuators B: Chemical 54: 3-15.

13. Homola J1 (2008) Surface plasmon resonance sensors for detection of chemical and biological species. Chem Rev 108: 462-493.

14. Bhatnagar K, Pathak A, Menke D, Cornish PV, Gangopadhyay K, et al. (2012) Fluorescence enhancement from nano-gap embedded plasmonic gratings by a novel fabrication technique with HD-DVD. Nanotechnology 23: 49520. 
15. Liu Z, Boltasseva A, Pedersen RH, Bakker R, Kildishev AV, et al. (2008) Plasmonic nanoantenna arrays for the visible. Metamaterials 2: 45-51.

16. Ni X, Emani NK, Kildishev AV, Boltasseva A, Shalaev VM (2012) Broadband light bending with plasmonic nanoantennas. Science 335: 427.

17. Liu Z, Steele JM, Srituravanich W, Pikus Y, Sun C, et al. (2005) Focusing surface plasmons with a plasmonic lens. Nano Lett 5: 1726-1729.

18. Ropers C, Neacsu CC, Elsaesser T, Albrecht M, Raschke MB, et al. (2007) Grating-coupling of surface plasmons onto metallic tips: a nanoconfined light source. Nano Lett 7: 2784-2788.

19. Ebbesen TW, Lezec HJ, Ghaemi HF, Thio T, Wolff PA (1998) Extraordinary optical transmission through sub-wavelength hole arrays. Nature 39: 667-669.

20. Lévêque G, Martin OJ (2006) Optical interactions in a plasmonic particle coupled to a metallic film. Opt Express 14: 9971-9981.

21. Mock JJ, Hill RT, Degiron A, Zauscher S, Chilkoti A, et al. (2008) Distancedependent plasmon resonant coupling between a gold nanoparticle and gold film. Nano Lett 8: 2245-2252.

22. Mock JJ, Hill RT, Tsai YJ, Chilkoti A, Smith DR (2012) Probing dynamically tunable localized surface plasmon resonances of film-coupled nanoparticles by evanescent wave excitation. Nano Lett 12: 17571764.

23. Shannon CE (1949) Communication in the Presence of Noise. Proceedings of the IRE 37: 10-12.

24. Ha T, Rasnik I, Cheng W, Babcock HP, Gauss GH, et al. (2002) Initiation and re-initiation of DNA unwinding by the Escherichia coli Rep helicase. Nature 419: 638-641.

25. Xiao M, Selvin PR (2001) Quantum yields of luminescent lanthanide chelates and far-red dyes measured by resonance energy transfer. J Am Chem Soc 123: 7067-7073.

26. Gryczynski I, Malicka J, Gryczynski Z, Lakowicz JR (2004) Radiative decay engineering 4. Experimental studies of surface plasmon-coupled directional emission. Anal Biochem 324: 170-182.

27. Darr CM, Korampally V, Chen B, Gangopadhyay K, Gangopadhyay S (2014) Plasmonic-enhanced conjugated polymer fluorescence chemosensor for trace nitroaromatic vapor. Sensors and Actuators B: Chemical 202: 1088-1096. 
28. Prescott B, Steinmetz W, Thomas GJ Jr (1984) Characterization of DNA structures by laser Raman spectroscopy. Biopolymers 23: 235-256.

29. Mayama H, Nomura SM, Oana H, Yoshikawa K (2000) Self-oscillating polymer chain. Chemical Physics Letters 330: 361-367.

30. Liedl T, Simmel FC (2005) Switching the conformation of a DNA molecule with a chemical oscillator. Nano Lett 5: 1894-1898.

31. Baumann CG, Smith SB, Bloomfield VA, Bustamante C (1997) Ionic effects on the elasticity of single DNA molecules. Proc Natl Acad Sci U S A 94: 6185-6190.

32. Richmond TJ, Davey CA (2003) The structure of DNA in the nucleosome core. Nature 423: 145-150.

33. Wong OK, Guthold M, Erie DA, Gelles J (2008) Interconvertible lac repressor- DNA loops revealed by single-molecule experiments. PLoS Biol 6: e232.

34. Rouzina I, Bloomfield VA (1998) DNA bending by small, mobile multivalent cations. Biophys J 74: 3152 3164.

35. Wang M, Kang Q, Ben-Naim E (2010) Modeling of electrokinetic transport in silica nanofluidic channels. Anal Chim Acta 664: 158-164.

36. Karnik R, Fan R, Yue M, Li D, Yang P, et al. (2005) Electrostatic control of ions and molecules in nanofluidic transistors. Nano Lett 5: 943-948.

37. Xuan X, Sinton D, Li D (2004) Thermal end effects on electroosmotic flow in a capillary. International Journal of Heat and Mass Transfer 47: 3145-3157.

38. Donner JS, Baffou G, McCloskey D, Quidant R (2011) Plasmon-assisted optofluidics. ACS Nano 5: 54575462.

39. Sugimoto N, Nakano S, Katoh M, Matsumura A, Nakamuta H, et al. (1995) Thermodynamic parameters to predict stability of RNA/DNA hybrid duplexes. Biochemistry 34: 11211-11216. 


\subsubsection{Chapter 4: Preparation of Ribosomes for smFRET Studies: A Simplified}

\section{Approach}

As found in: *Shebl, B., *Menke, D. E., Pennella, M., Poudyal, R. R., Burke, D. H., \& Cornish, P. V. (2016). Preparation of ribosomes for smFRET studies: A simplified approach. Archives of biochemistry and biophysics, 603, 118-130.

* Co-first Authors are considered to have contributed equally to the manuscript

\subsubsection{Abstract}

During the past decade, single-molecule studies of the ribosome have significantly advanced our understanding of protein synthesis. The broadest application of these methods has been towards the investigation of ribosome conformational dynamics using single-molecule Förster resonance energy transfer (smFRET). The recent advances in fluorescently labeled ribosomes and translation components have resulted in success of smFRET experiments. Various methods have been employed to target fluorescent dyes to specific locations within the ribosome. Primarily, these methods have involved additional steps including subunit dissociation and/or full reconstitution, which could result in ribosomes of reduced activity and translation efficiency. In addition, substantial time and effort are required to produce limited quantities of material. To enable rapid and large-scale production of highly active, fluorescently labeled ribosomes, we have developed a procedure that combines partial reconstitution with His-tag purification. This allows for a homogeneous single-step purification of mutant ribosomes and subsequent integration of labeled proteins. Ribosomes produced with this method are shown to be as active as ribosomes purified using classical methods. While we have focused on two labeling sites in this report, the method is generalizable and can in principle be extended to any non-essential ribosomal protein.

\subsubsection{Introduction}

A significant component of the central dogma involves the translation of the genetic code to proteins, which function in many structural and enzymatic roles. Translation is a ubiquitous process that is shared across all domains of life. The platform upon which proteins are made is the ribosome, which is assisted by various accessory factors in an elegant and complex mechanism. At $\sim 2.5 \mathrm{MDa}$, the bacterial 
ribosome is one of the largest macromolecular complexes of the cell. The ribosome, composed of two subunits (large 50S and small 30S), is assembled from an interwoven mesh of ribosomal RNA (rRNA) and a total of 52 ribosomal proteins. Given the high degree of homology and absolute conservation of specific regions within the structure, the bacterial ribosome has served as a model for studying translation and more generally in understanding the structure and function of cellular macromolecular complexes [1]. Since the elucidation of the first atomic resolution ribosome structures in the early 2000 s, translation has gained an increased interest in many fields including X-ray crystallography, cryo-electron microscopy, and single-molecule biophysics [1]; [2]; [3]; [4]. These techniques among others have provided an expanded understanding of the different kinetics and conformational dynamics of translation machinery. In particular, single molecule Förster Resonance Energy Transfer (smFRET) experiments have allowed researchers to tap into uncharted territories in ribosome function and have uncovered key aspects of the mechanism of translation [5]. Due to the inherent nature of smFRET experiments and notably the direct detection of relative distance changes, bulk averaging can be overcome revealing real time visualization of complex pathways and conformational changes of the ribosome and translation factors. Thus, smFRET studies performed on the ribosome provide powerful insight, which complements structural studies [6]; [7]; [8]; [9]; [10]; [11].

In early pioneering smFRET experiments on the ribosome, transfer RNAs (tRNAs) were fluorescently labeled and were observed to spontaneously fluctuate between distinct conformational states [7]; [12]. These experiments confirmed previous results showing that tRNAs transition between a classical and hybrid state during translation and showed that P and A site tRNA can transition to the hybrid state independently [7]; [12]; [13]. While these experiments with labeled tRNAs have provided essential knowledge relative to the mechanism of translation, experiments with fluorescent dyes directly attached to the ribosome were necessary to gain insight into the conformational flexibility of the ribosome. Hybridlabeling schemes emerged involving the labeling of a ribosomal protein with another dye on P-site tRNA [6] or on a translation factor [14]; [15]. In separate experiments, dual labeling of the ribosome was accomplished by either labeling ribosomal proteins or by hybridizing fluorescently labeled DNA to rRNA extensions within the ribosome [16]; [17]. These studies collectively provided researchers with the ability to directly observe conformational changes occurring during translation and how these changes are 
influenced by differences in tRNA amino acylation states and the presence of antibiotics and other factors [8]; [9]; [18]; [19]. Labeling the ribosome in this manner poses a substantial technical challenge due to the complexity of the structure and the necessity, in some cases, to perform partial or full reconstitution of the ribosome [20]; [21]; [22]. Additionally, the chosen labeling scheme needs to be sufficient to produce a homogenous sample with minimal disruption to the biological structure, limiting the perturbation to functional activity.

Driven by the need for highly active, fluorescently labeled ribosomes, different laboratories have adapted standard methodologies and developed a wide range of purification protocols. Choosing a specific purification protocol is application-dependent. The classical purification approach involves a series of ultracentrifugation steps to isolate ribosomes from cellular debris and other macromolecular complexes [23]. While this procedure is sufficient to generate pure ribosomal subunits, the difficulty has been to target a dye molecule to a specific protein in the large ribosome complex. Two separate approaches have been employed to approach this problem. First, researchers have been able to specifically knockout individual non-essential ribosomal proteins generating knockout strains. The protein is then purified separately, fluorescently labeled and reconstituted into the ribosome purified from the knockout strain [17]. However, in the case of essential proteins, genetic knockouts are lethal. The entire set of ribosomal proteins of the target subunit has to be purified individually, fluorescently labeled and fully reconstituted into a ribosomal subunit [20]; [21]. This has only found success in the context of the 30S subunit and involves the purification of 20 small subunit ribosomal proteins and the $16 \mathrm{~S}$ rRNA. An alternate approach to labeling ribosomal proteins has been to label rRNA by first extending the rRNA to allow for this sequence to be hybridized to a short fluorescently labeled DNA oligonucleotide [16]. In both approaches, one or several ultracentrifugation steps are still necessary, which requires a significant time investment. Hence, there is a necessity for a more cost effective and timesaving approach that ultimately results in highly active ribosomes. For further discussion on the different labeling schemes used we refer the reader to the following reviews [5]; [24]; [25]; [26].

By exploiting the physical properties of ribosomes, techniques such as size exclusion chromatography [27] and hydrophobic interaction chromatography [28] have been used to purify ribosomes. Affinity-tag based chromatography [29]; [30]; [31]; [32]; [33]; [34] has gained much popularity 
among purification techniques considering the facility and specificity of the approach. Affinity-tag based methods for purifying ribosomes bearing mutations involve overexpression of a tagged ribosomal protein or rRNA and purifying the tagged ribosomes from a background of native/wild-type ribosomes [34]; [35]; [36]. A more recent approach for non-lethal mutants involves introducing a specific tag within the bacterial genome to the ribosomal L7/12 stalk producing a homogenous population of tagged ribosomes [31].

Here, we report a new hybrid method for producing fluorescently labeled ribosomes of high activity and larger yields than previous methods (Fig. 1). We combine both the ease of His-tag purification with the ability to genetically knock out ribosomal proteins in the same strain. This provides the benefit of producing labeled ribosome samples in significantly less time and provides the opportunity to extend this methodology to labeling other non-essential ribosomal proteins. Further, we have extensively characterized fluorescently labeled ribosomes produced using this technique and show that they are as active as ribosomes purified using classical purification methods.

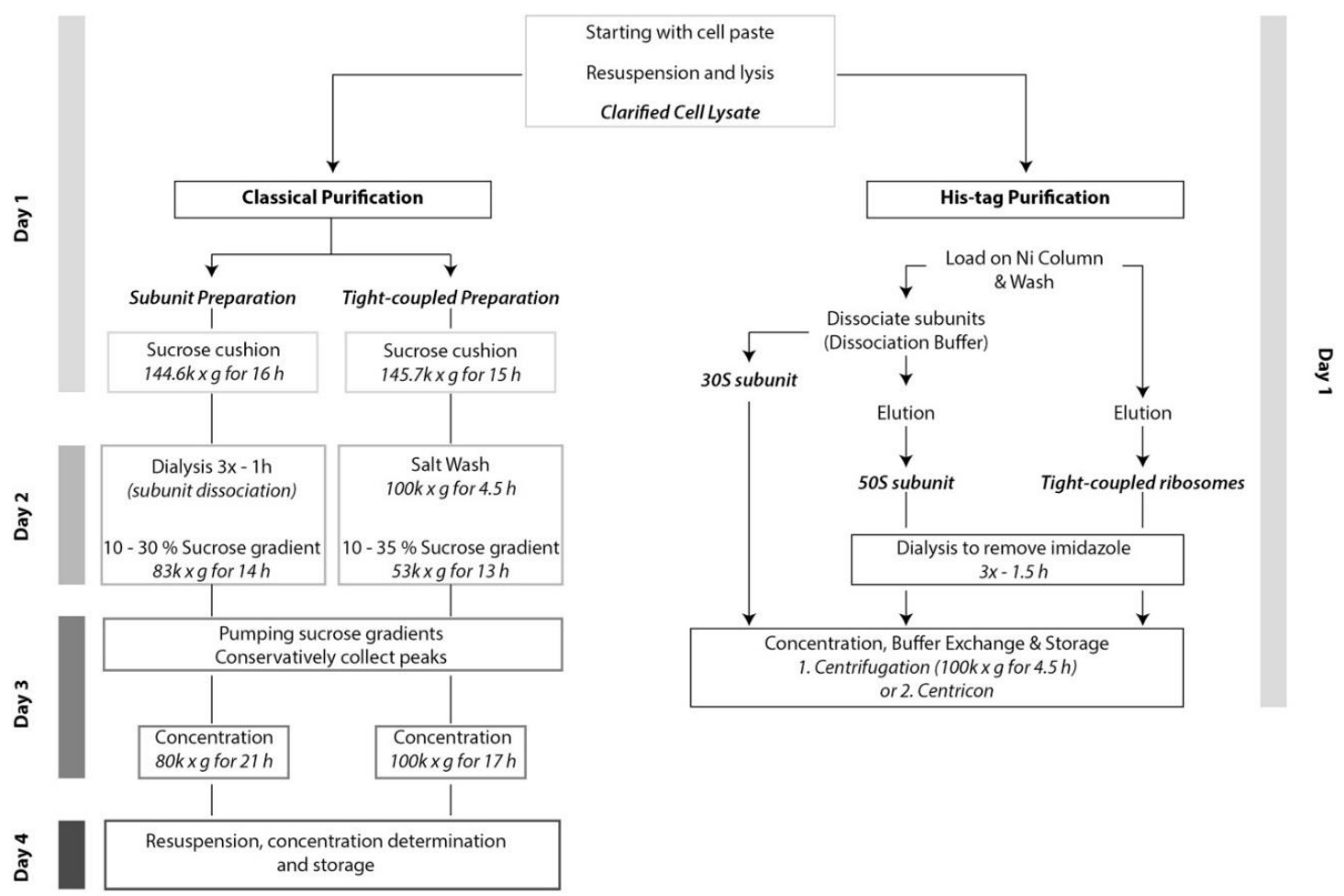

\subsubsection{Figure 25 Timelines for purifying ribosomes}

Figure 1: A Timeline for purifying ribosomes using a classical purification protocol versus a His-tagged purification protocol. 


\subsubsection{Materials and methods}

\section{Preparation of linear DNA cassette for L7/L12 His-tag}

The procedure was adapted from Ederth et al. [31]. pET-28a was used as a template to amplify the kanamycin (kan) -resistant cassette by primers L12Kanfor1 and PolBKanrev1 (Table 1). The amplified DNA fragment from the first PCR was purified and used as the template for the second PCR using primers L12homofor and polBhomorev (Table 1). The final DNA fragment has 47 nucleotides homologous to the 3' end of the E. coli rplL gene, minus the stop codon. A $(\mathrm{CAC})_{6}$ repeat coding for six Histidines was inserted before a stop codon TAA. The final DNA fragment has 45 nucleotides homologous to the DNA sequence after the $r p l L$ gene. The final PCR product was purified and used as linear DNA fragment for lambda Red recombineering (see below).

\subsubsection{Table 3 Oligonucleotides used}

\begin{tabular}{|c|c|}
\hline Table 1. & Oligonucleotides used. \\
\hline Name & Sequence \\
\hline L12Kanfor1 & $\begin{array}{l}\text { CGCTGAAGTTGAAGTTAAACACCACCACCACCACCACTAAAAACAGTAATAC } \\
\text { AAGGG }\end{array}$ \\
\hline $\begin{array}{l}\text { PolBKanrev } \\
1\end{array}$ & CTGCAACCGGAAGGGTTGGCTTAGAAAAACTCATCGAGCATC \\
\hline $\begin{array}{l}\text { L12homofo } \\
\text { r }\end{array}$ & CAGAAGCACTGAAAAAAGCTCTGGAAGAAGCTGGCGCTGAAGTT \\
\hline $\begin{array}{l}\text { PolBhomor } \\
\text { ev }\end{array}$ & GTCACCAGCCATCAGCCTGATTTCTCAGGCTGCAACCGGAAGGG \\
\hline S6forCat1 & $\begin{array}{l}\text { CAGGAGGCTGAATAATCCGTAAGGAGCAATTCGATGGAGAAAAAAATCACTG } \\
\text { G }\end{array}$ \\
\hline S6revCat 1 & $\begin{array}{l}\text { CGGTGCCGGACAACACCAGACGGTTGGTCATCAGAAATTAATGAATCGGCCA } \\
\text { ACG }\end{array}$ \\
\hline S6for2 & $\begin{array}{l}\text { TTTAACACGTTCCTTGCCTCCCCGGGATTCGGCTGACCCAGACAGGAGGCTGA } \\
\text { ATAATCC }\end{array}$ \\
\hline S6rev2 & $\begin{array}{l}\text { GGAATTCCTGATGGACTGACCTTTCGAAGGGGAGCCCTGCACACGGTGCCGG } \\
\text { ACAACACC }\end{array}$ \\
\hline
\end{tabular}

\section{Construction of the parent His-tagged strain}

Plasmid pKD46, a lambda Red recombinase expression plasmid under the araBAD promoter, was transformed into BW25113 [37]. A single colony was inoculated and electrocompetent cells were prepared by growing at $30{ }^{\circ} \mathrm{C}$ with 1-arabinose. The linear DNA fragment carrying the kan-resistance gene with homologous ends to $r p l L$ was electroporated into the competent cells. Colonies that successfully recombined with the linear DNA fragment were selected on kan plates at $37{ }^{\circ} \mathrm{C}$. The positive insertion colonies were verified by PCR amplification and DNA sequencing of the insertion site. The resulting strain 
with the correct insertion of a C-terminal (His) $)_{6}$ tag on ribosomal protein L7/L12 was named BD0012 (Table 2).

\subsubsection{Table 4 List of strains used}

\begin{tabular}{|l|l|}
\hline Table 2. & \\
\hline List of strains used. & \\
\hline Strain description & Strain name \\
\hline BW25113 $-\Delta$ S6 from the Keio collection & JW4158 \\
\hline BW25113 $-\Delta$ L9 from the Keio collection & JW4161 \\
\hline BW25113 $-\Delta$ S6/ $\Delta$ L9 & BD6900 \\
\hline BW25113 $-\Delta$ S6 - L12 His-tagged & BD6012 \\
\hline BW25113 - L12 His-tagged & BD0012 \\
\hline BW25113 $-\Delta$ L9 - L12 His-tagged & BD0912 \\
\hline BW25113 $-\Delta$ S6/ $\Delta$ L9 - L12 His-tagged & BD6912 \\
\hline Constrution of the knockout strains & \\
\hline
\end{tabular}

\section{Construction of the knockout strains}

\section{Single knockout mutants}

The $\Delta \mathrm{S} 6$ (JW4158) and $\Delta \mathrm{L} 9$ (JW4161) knockout mutants were purchased from the Keio collection and used for the purification of $\Delta \mathrm{S} 630 \mathrm{~S}$ and $\Delta \mathrm{L} 950 \mathrm{~S}$ subunits (Table 2) in the classical subunit purification protocol (see below).

\section{Generation of a His-tagged double ribosomal protein knockout}

The $\Delta \mathrm{L} 9$ knockout mutant (JW4161) transformed with pCP20 [37] was cured of its kan-resistance cassette. P1 Phage transduction (see below) was used to move the L7/L12 His-tagged mutation from BD0012 to the cured $\Delta \mathrm{L} 9$ mutant. The resultant strain ( $\Delta$ L9 L7/L12 His-tagged mutant) was named BD0912 (Table 2). Direct generation of a $\Delta$ L9 $\Delta$ S6 L7/L12 His-tagged mutant strain was not possible due to the close proximity of S6 $(r p s F)$ and L9 gene $(r p I I)$ in the genome and the limited packaging capacity of the P1 phage. A new S6 deletion cassette was designed to replace $r p s F$ with a chloramphenicol-resistance cassette in a similar fashion as performed for the L7/L12 His-tag (see above) using primers S6forCat1/S6revCat1 and S6for2/S6rev2 (Table 1). The transformation of the $\Delta \mathrm{S} 6$ chloramphenicoldeletion cassette and expression of the pKD46 system in the $\Delta \mathrm{L} 9 \mathrm{~L} 7 / \mathrm{L} 12$ His-tagged strain resulted in a new $\Delta$ S6 $\Delta$ L9, L7/L12 His-tag strain (BD6012) (Table 2) with antibiotic resistance cassettes for kan and chloramphenicol (cam).

\section{P1 phage transduction}


The P1 vir phage used was a gift from Bob Sauer (MIT). A detailed protocol can be accessed online (http://openwetware.org/wiki/Sauer:P1vir_phage_transduction). In brief, P1 vir phage was used in the transfer of genetic material from a donor strain to a recipient strain. This is accomplished by the unique mechanism by which P1 phage packages random fragments of the donor strain genome, $\sim 90,000 \mathrm{bp}$. The donor strain was transduced with P1 vir phage and left to shake at $37^{\circ} \mathrm{C}$ for $1-3 \mathrm{~h}$. Chloroform was added to complete cell lysis producing a new phage lysate. The clarified phage lysate was reintroduced to the original donor strain. A minimum of two passages was performed to clear the phage of previously held genetic material. The resultant phage was used to infect the recipient strain for $1 \mathrm{~h}$ at $37^{\circ} \mathrm{C}$ after which sodium citrate was added to prevent the reinfection of phage into Escherichia coli. Colonies were screened for the presence of the donor's antibiotic-resistance marker.

\section{Purification of ribosomes}

\section{Classical purification of tight-coupled ribosomes and subunits}

Tightly-coupled 70S ribosomes and/or purified 50S and 30S subunits were purified via preparative sucrose gradient density ultracentrifugation of clarified cell lysate from either wild-type (WT) or mutant strains as described previously [17]; [20]; [38]. For a detailed protocol and a list off buffers used (Table S1), refer to the supplementary information.

\section{Affinity purification of tight-coupled His-tagged ribosomes}

A fresh overnight culture of $E$. coli BW21153, bearing the desired mutation(s) (Table 2), was used to inoculate LB with $\operatorname{kan}(50 \mu \mathrm{g} / \mathrm{mL})$. The culture was grown with shaking at $37^{\circ} \mathrm{C}$ until an $\mathrm{OD}_{600}$ of 0.6 . The culture was left to cool on ice with occasional shaking. Cells were harvested by centrifugation in a Sorvall SLC-6000 at 3000 RPM for 20 min. The cell-pellet was briefly rinsed with cold lysis buffer G and stored at $-80^{\circ} \mathrm{C}$. The cell-pellet was resuspended in lysis buffer $\mathrm{G}$ and lysed using a French Press. The cell debris was spun down twice in a Sorvall SS-34 at 12,000 RPM for $20 \mathrm{~min}$ at $4{ }^{\circ} \mathrm{C}$. The clarified lysate was filtered and loaded onto a GE Healthcare Hisprep FF 16/10 equilibrated with buffer G. The column was washed with 5 column volumes of buffer $\mathrm{G}$ to baseline the $\mathrm{A}_{260}$ reading and to ensure the complete clearance of the flow-through. Next, non-specific binders were washed off with wash buffer $\mathrm{H}$ until the $\mathrm{A}_{260}$ went back to the baseline. The sample was eluted with elution buffer I. The elution fractions were pooled and dialyzed immediately $3 \times$ into $1 \mathrm{~L}$ of buffer $\mathrm{G}$ for $30 \mathrm{~min}$ each at $4{ }^{\circ} \mathrm{C}$ to remove the imidazole. 
The ribosomes were concentrated by ultracentrifugation at $150,000 \times \mathrm{g}$ for $2 \mathrm{~h}$ at $4{ }^{\circ} \mathrm{C}$. The pelleted ribosomes were resuspended in buffer B for BD0012 ribosomes or buffer S for the BD6912 ribosomes. Ribosomes were flash frozen and stored at $-80{ }^{\circ} \mathrm{C}$.

\section{Affinity purification of $50 S$ and $30 S$ subunits}

Individual subunits can be purified using affinity purification via minor modifications to the previous section. The clarified lysate was loaded on the GE Healthcare Hisprep FF 16/10. After the $260 \mathrm{~nm}$ absorbance baselined, the $30 \mathrm{~S}$ was eluted by dissociation from the $50 \mathrm{~S}$ with buffer $\mathrm{J}$. The column was washed with wash buffer $\mathrm{H}$ and the His-tagged 50S subunit was eluted using elution buffer I. Dialysis to remove imidazole was performed as described in the previous section. The purified subunits were concentrated by centrifugation at $80,000 \times \mathrm{g}$ for $21 \mathrm{~h}$ at $4{ }^{\circ} \mathrm{C}$ and resuspended in the appropriate buffer or exchanged and concentrated with a Millipore Centricon ${ }^{\circledR}$.

\section{Cloning and purification of ribosomal proteins}

$r p s F$ (S6 gene) and rpII (L9 gene) were cloned into pET-11a. Site-directed mutagenesis was performed to introduce a single cysteine at residues D41C for S6 and N11C for L9 [17]. The S6 D41C mutant was expressed in E. coli BL21 (DE3). Cultures were induced for $4 \mathrm{~h}$ with $1 \mathrm{mM}$ IPTG. Cells were pelleted in an SLC-6000 Sorvall rotor at $3000 \times \mathrm{g}$ for $20 \mathrm{~min}$ and stored at $-20{ }^{\circ} \mathrm{C}$. Cells were resuspended in lysis buffer $\mathrm{K}$ and lysed by French Press. The lysate was then clarified at 12,000 RPM for 15 min in a Sorvall SS-34 rotor at $4{ }^{\circ} \mathrm{C}$. The pellet containing inclusion bodies was then dissolved in buffer L. After the solubilization of the inclusion bodies, the sample was dialyzed three times against buffer $\mathrm{M}$ at $4{ }^{\circ} \mathrm{C}$. The sample was then clarified by centrifugation at 12,000 RPM for $15 \mathrm{~min}$ in a Sorvall SS-34 rotor at $4{ }^{\circ} \mathrm{C}$ and filtered through a $0.8 \mu \mathrm{m}$ cellulose acetate syringe filter. The protein was purified by a 100/10 Resource Q anion exchange column with a $120 \mathrm{~mL} 0-22 \%$ linear gradient of Buffer L in buffer M. S6 D41C was further purified by Hiload 26/60 Superdex prep grade in buffer M. The purified protein was then dialyzed into S6 labeling buffer N, concentrated and flash frozen.

The L9 N11C mutant was expressed in E. coli BL21 (DE3) by growth in 1.5L LB to an $\mathrm{OD}_{600}$ of $0.4-0.8$ at $37^{\circ} \mathrm{C}$. Cultures were induced for $4 \mathrm{~h}$ with $1 \mathrm{mM}$ IPTG. Cells were pelleted in an SLC-6000 Sorvall rotor at $3000 \times \mathrm{g}$ for $20 \mathrm{~min}$ and stored at $-20{ }^{\circ} \mathrm{C}$. Cells were resuspended in lysis buffer $\mathrm{K}$ and lysed by French Press. The lysate was then clarified at 12,000 RPM for 15 min in a Sorvall SS-34 rotor at 
$4{ }^{\circ} \mathrm{C}$. The pellet containing inclusion bodies was then dissolved in $10 \mathrm{~mL}$ of buffer $\mathrm{O}$. After solubilizing the inclusion bodies, the sample was dialyzed $3 \times$ against buffer $\mathrm{P}$ at $4{ }^{\circ} \mathrm{C}$. The sample was then clarified by centrifugation at 12,000 RPM for $15 \mathrm{~min}$ in an SS-34 rotor at $4{ }^{\circ} \mathrm{C}$ and filtered through a $0.8 \mu \mathrm{m}$ cellulose acetate syringe filter. The protein was purified by a 100/10 Resource S cation exchange column with a $120 \mathrm{~mL} 0-100 \%$ linear gradient of buffer O in buffer P. L9 N11C was further purified by Hiprep Butyl FF 16/10 using a $100-0 \%$ ammonium sulfate gradient in buffer Q. Protein obtained by this method was then dialyzed into L9 labeling buffer R, concentrated and frozen.

\section{Fluorescent labeling of ribosomal proteins}

50-200 $\mu \mathrm{M}$ of S6 D41C or L9 N11C was mixed with 2-fold excess of tris (2-carboxyethyl) phosphine (TCEP) and incubated at room temperature for $30 \mathrm{~min}$ [17]; [20]. Twenty-fold excess of sulfo$\mathrm{Cy} 3 / \mathrm{Cy} 5$ maleimide (Lumiprobe) was then added to the mixture and incubated for $2-4 \mathrm{~h}$ at room temperature or $4{ }^{\circ} \mathrm{C}$ overnight. The reaction was quenched by addition of $6 \mathrm{mM} \beta$-mercaptoethanol ( $\left.\beta \mathrm{ME}\right)$. A NAP-5 column (GE Healthcare Life Sciences) was used to separate the labeled protein from free dye. Fractions containing the labeled protein were pooled together and exchanged into either S6 reassociation buffer S or L9 reassociation buffer T. Purified, labeled protein was run on 16\% SDS-PAGE. The attachment of a fluorescent dye to a small ribosomal protein results in a small mass shift in the gel. Therefore, labeling efficiency was determined by a combination of fluorescent imaging on a Biorad ChemiDoc and Coomassie-staining. Labeling efficiencies near 100\% were obtained for both S6 and L9. Reconstitution and assembly of ribosomes

\section{Assembly of S6/L9 classically purified ribosomes}

1.2 nmoles of $\triangle \mathrm{S} 630 \mathrm{~S}$ and $1.8 \mathrm{nmoles}$ of S6 D41C sulfo-Cy5 were assembled together in S6 reassociation buffer $\mathrm{S}$ at $37^{\circ} \mathrm{C}$ for $15 \mathrm{~min} .1 .2$ nmoles of $\Delta \mathrm{L} 950 \mathrm{~S}$ and 1.8 nmoles of L9 N11C sulfo-Cy3 were assembled together in $\mathrm{L} 9$ reassociation buffer $\mathrm{T}$ at $37{ }^{\circ} \mathrm{C}$ for $15 \mathrm{~min}$. Both subunits were then exchanged into 70S reassociation buffer U using a $100 \mathrm{~K}$ MWCO Millipore Centricon ${ }^{\circledR}$. The reconstituted $30 \mathrm{~S}$ and $50 \mathrm{~S}$ subunits were heat activated at $42^{\circ} \mathrm{C}$ for $10 \mathrm{~min}$. The reconstituted subunits were mixed together and associated at $37{ }^{\circ} \mathrm{C}$ for $20 \mathrm{~min}$. The samples were layered on a $10-30 \%$ sucrose gradient in $70 \mathrm{~S}$ reassociation buffer $\mathrm{U}$. The gradients were centrifuged at $71,000 \times \mathrm{g}$ for $14 \mathrm{~h}$ in an SW41 rotor. The $70 \mathrm{~S}$ peak was conservatively collected and dialyzed into $70 \mathrm{~S}$ reassociation buffer $\mathrm{U}$ by using a $100 \mathrm{~K}$ 
MWCO Millipore Centricon ${ }^{\circledR}$. The reassociation efficiency was determined by absorption measurements at 260, 550 and 650 . Reassociation efficiencies near $\sim 100 \%$ were obtained. Purified ribosomes were diluted, aliquoted and frozen at $-80{ }^{\circ} \mathrm{C}$.

\section{Assembly of S6/L9 His-tagged ribosomes}

1.2 nmoles of $\Delta \mathrm{S} 6 / \Delta \mathrm{L} 9 \mathrm{~L} 12$ His-tagged $70 \mathrm{~S}$ and 1.8 nmoles of L9 N11C sulfo-Cy3 were assembled together in $\mathrm{L} 9$ reassociation buffer $\mathrm{T}$ at $37{ }^{\circ} \mathrm{C}$ for $15 \mathrm{~min}$. The ribosomes were then exchanged into S6 reassociation buffer S. 1.8 nmoles of S6 D41C sulfo-Cy5 were added and the mixture was incubated at $37^{\circ} \mathrm{C}$ for $15 \mathrm{~min}$. The reassociated ribosomes were then exchanged into $70 \mathrm{~S}$ reassociation buffer U by using a $100 \mathrm{~K}$ MWCO Millipore Centricon ${ }^{\circledR}$. Reassociation efficiencies were assessed as mentioned above. Purified ribosomes were aliquoted and flash frozen at $-80{ }^{\circ} \mathrm{C}$.

Measurement of the growth rate

All of the generated strains (Table 2) and WT parent strains were grown from overnight cultures. The cultures were grown with and without antibiotics ( $\mathrm{kan} 50 \mu \mathrm{g} / \mathrm{mL}$ and cam $35 \mu \mathrm{g} / \mathrm{m}$ ). The $\mathrm{OD}_{600}$ was monitored using a Biotek Synergy 2 Multi-Mode Reader over the course of $10 \mathrm{~h}$ with Greiner Bio-One 96 well plates.

\section{Sucrose density-gradient analysis}

$300-1200$ pmoles of ribosomal 70S, 50S or 30S subunits were layered onto a 10-30\% sucrose gradient in buffer $\mathrm{W}$ in an SW41 rotor at $71,000 \times \mathrm{g}$ for $14 \mathrm{~h}$ at $4{ }^{\circ} \mathrm{C}$. The gradients were pumped with $40 \%$ sucrose using a Brandel density gradient fractionator while monitoring the $\mathrm{A}_{260}$ absorbance.

Assessment of labeled protein incorporation

Purified, reconstituted ribosomes were assayed on a 16\% SDS-PAGE. Fluorescent images were taken using a Biorad ChemiDoc to detect fluorescently labeled proteins (Cy3: Excitation 548/Emission 563, and Cy5: Excitation 646/Emission 662). The gel was Coomassie-stained for total protein.

\section{Preparation of translation factors, mRNA, and tRNAs}

$\mathrm{tRNA}^{\mathrm{fMet}}, \mathrm{tRNA}^{\mathrm{Phe}}$ and tRNA ${ }^{\text {Tyr }}$ were purchased from MP Biomedicals, tRNA probes and Sigma, respectively. fMet-tRNA ${ }^{\mathrm{fMet}}$ and NAc-Phe-tRNA ${ }^{\text {Phe }}$ were aminoacylated using DEAE-purified S100 enzymes. Aminoacylated tRNAs were purified from the charging reaction and the extent of aminoacylation was verified by acid gel electrophoresis [39]; [40]; [41]. The messenger ribonucleic acid (mRNA) used for 
smFRET, m291, is a variant of T4 bacteriophage gene 32 (T4gp32) [42]. We used m301, a variant of m291, for filter binding, where the ATG codon was replaced with UAC [17]; [42]. The mRNA was in vitro transcribed from a PCR template with an upstream T7 promoter and purified on denaturing PAGE [43]; [44]. Elongation Factor-G (EF-G) was cloned from MRE600 into an expression vector with a Cterminal $(\mathrm{His})_{6}$ - tag. After overexpression in BL21, EF-G was purified on a GE Healthcare Hisprep FF $16 / 10$ column and eluted in $250 \mathrm{mM}$ imidazole. Purified EF-G was buffer exchanged to storage buffer V and stored at $-80{ }^{\circ} \mathrm{C}$ [45].

\section{tRNA-binding and puromycin reactivity assay}

The assay was performed as described previously with minor modifications [5]; [46]. To assay tRNA binding in the P-site, ribosomes were assembled with m301 and N-Ac- $\left[{ }^{3} \mathrm{H}\right] \mathrm{Phe}^{-} \mathrm{RNA}{ }^{\mathrm{Phe}}$ and incubated at $37^{\circ} \mathrm{C}$ for $20 \mathrm{~min}$. All samples were bound to a nitrocellulose membrane (Millipore HAWP $0.45 \mu \mathrm{m})$ and washed $3 \times$ with buffer $\mathrm{X}$ and the membranes were left to dry. All membranes were counted in scintillation cocktail (Bio-Safe II ${ }^{\mathrm{TM}}$, RPI). For assessing tRNA binding in the A-site, ribosomes were incubated with tRNA ${ }^{\text {Tyr }}$ at $37{ }^{\circ} \mathrm{C}$ for 20 min, followed by a second incubation with N-Ac- $\left[{ }^{3} \mathrm{H}\right]$ Phe-tRNA ${ }^{\text {Phe }}$ at $37^{\circ} \mathrm{C}$ for $20 \mathrm{~min}$. To assay peptidyl-transferase center (PTC) activity, ribosomes were assembled with N-Ac- $\left[{ }^{3} \mathrm{H}\right]$ Phe-tRNA ${ }^{\text {Phe }}$ as mentioned above, and incubated with puromycin, an antibiotic, to a final concentration of $1 \mathrm{mM}$ at $37^{\circ} \mathrm{C}$ for $10 \mathrm{~min}$. The reaction was quenched with an equivalent volume of $0.3 \mathrm{M} \mathrm{NaOAc}\left(\mathrm{pH} 5.4\right.$ ), saturated with $\mathrm{MgSO}_{4}$. Puromycin-bound $\left[{ }^{3} \mathrm{H}\right]$ Phe was extracted using $1 \mathrm{~mL}$ of ethyl acetate and $800 \mu \mathrm{l}$ of the organic phase was counted. Finally, EF-G translocation efficiency was assayed by preassembling the ribosome with $\mathrm{tRNA}^{\mathrm{Tyr}}$ and N-Ac- $\left[{ }^{3} \mathrm{H}\right]$ Phe-tRNA ${ }^{\text {Phe }}$ and incubating with EFG/GTP at $37^{\circ} \mathrm{C}$ for $15 \mathrm{~min}$. Subsequently, puromycin was added as mentioned above to assess the P-site bound N-Ac- $\left[{ }^{3} \mathrm{H}\right]$ Phe-tRNA ${ }^{\text {Phe }}$ fraction.

\section{Preparation of ribosomal complexes for smFRET experiments}

All ribosome complexes used for smFRET imaging were assembled in buffer Y (20 mM HEPES$\mathrm{KOH} \mathrm{pH}$ 7.5, $6 \mathrm{mM} \mathrm{MgCl}_{2}, 150 \mathrm{mM} \mathrm{NH} \mathrm{H}_{4} \mathrm{Cl}, 6 \mathrm{mM} \beta \mathrm{ME}, 2 \mathrm{mM}$ spermidine, and $0.1 \mathrm{mM}$ spermine) [8]. The presence of polyamines, spermidine and spermine, stabilizes ribosomal subunit association even at low $\mathrm{Mg}^{2+}$ concentrations [47]; [48]. Ribosomal complexes carrying a single tRNA bound to the P-site were assembled by incubating $1 \mu \mathrm{M}$ S6/L9 labeled ribosomes with $2 \mu \mathrm{M}$ m291, pre-annealed to biotin-labeled 
DNA primer (5' biotin, CTTTATCTTCAGAAGAAAAACC-3', Integrated DNA Technologies), and $2 \mu \mathrm{M}$ tRNA (tRNA ${ }^{\mathrm{fMet}}$ or fMet-tRNA ${ }^{\mathrm{fMet}}$ ) at $37^{\circ} \mathrm{C}$ for $20 \mathrm{~min}$. The pre-translocation complexes were assembled by incubation of $1 \mu \mathrm{M}$ S6/L9 labeled ribosomes with $2 \mu \mathrm{M} \mathrm{m} 291$ pre-annealed to biotin-labeled DNA primer, and $2 \mu \mathrm{M}$ tRNA ${ }^{\mathrm{fMet}}$, at $37^{\circ} \mathrm{C}$ for 20 min followed by a second incubation with $2 \mu \mathrm{M} \mathrm{N}$-Ac-PhetRNA ${ }^{\text {Phe }}$ at $37^{\circ} \mathrm{C}$ for $20 \mathrm{~min}$.

Quartz slides were cleaned with acetone, methanol and $1 \mathrm{M} \mathrm{KOH}$ in sequence [25]; [49]; [50]; [51]. The surface was passivated with a mixture of m-PEG - succinimidyl valerate (MW 5000) and Biotin - PEG - succinimidyl valerate (MW 5000) (Laysan Bio Inc.) and pretreated with neutravidin $(0.2 \mathrm{mg} / \mathrm{mL})$ (Pierce). Ribosomal complexes were diluted to a final concentration of $1 \mathrm{nM}$ and immobilized on a quartz slide. To minimize photobleaching, all samples were imaged in buffer Y supplemented with an oxygen-scavenging system; $0.8 \mathrm{mg} / \mathrm{mL}$ glucose oxidase (Sigma), $0.625 \% \beta$-d-glucose, $0.02 \mathrm{mg} / \mathrm{mL}$ catalase (Roche), and $1.5 \mathrm{mM}$ Trolox (Sigma).

\section{smFRET data acquisition and analysis}

smFRET measurements were acquired on an Olympus IX-71 microscope with a UPlanSApo 60XW 1.20 objective lens. A $532 \mathrm{~nm}$ laser (Spectra-Physics) was used for excitation of Cy3 via prism-type total internal reflection microscopy (TIRFM) [25]; [50]. The fluorescence emission was split in two pathways (Cy3, Cy5 emission) with a 630dexr dichroic mirror (Chroma). For visualizing the fluorescence signal, an Andor iXon ${ }^{\mathrm{EM}}+897$ EMCCD camera was used. Movies were recorded using Single (software downloaded from Taekjip Ha's lab website: https://cplc.illinois.edu/software/). The data was acquired at $100 \mathrm{~ms}$ for all constructs. A calibration image was acquired using crimson fluorescent FluoSpheres ${ }^{\circledR}$ (Invitrogen). This allows for mapping of the two channels and accounts for rescaling, rotation and shear distortion. The recorded movies were processed to extract data using custom IDL scripts (Exelisvis), which were subsequently analyzed using custom scripts in Matlab (Mathworks).

Selection criteria for valid traces included: choosing traces showing single step photobleaching for $\mathrm{Cy} 3$ and $\mathrm{Cy} 5$, the trace lasts for $>1 \mathrm{~s}$ and either showed a stable FRET or anti-correlated behavior. Traces showing positive correlation, fluctuating dye effect, an intensity less than 100 arbitrary units (au) or FRET states less than 0.15 were excluded. FRET histograms were constructed from traces fitting the set criteria. The contribution of each trace to the histogram was normalized to the length of the trace to ensure equal 
contribution to the final histogram [49]; [52]. Data were smoothed with a five-point window average. The histograms were fit to two Gaussian distributions using IGOR Pro (Wavemetrics).

\subsubsection{Results and discussion}

Through the experimental procedures as described in Materials and Methods, we constructed a mutant bacterial strain that contained a genetically encoded His-tag on the L7/L12 stalk and also contained genomic knockouts of both $r p s F$ and $r p l I$, which encode for ribosomal proteins S6 and L9, respectively. From this strain, ribosomes were purified through His-tag chromatography and partially reconstituted with fluorescently labeled S6 and L9 to generate S6 (Cy5)/L9 (Cy3) His-tagged ribosomes for use in fluorescence based studies. Due to the high conservation within the ribosome, concern for potential perturbations that may result from mutagenesis, and the partial reconstitution, a thorough assessment of the generated mutant strains and fluorescently labeled ribosomes was necessary. We assessed the impact of introducing a genomic tag on the vitality of the mutated strains and the quality of the purified ribosomes in terms of intactness, purity, and most importantly the biochemical activity related to protein synthesis. Finally, we assessed the feasibility of using the new construct in performing smFRET experiments. To compare these results with previous methods of preparing fluorescently labeled ribosomes, we purified 30S and 50S subunits separately using ultracentrifugation, partially reconstituted with fluorescently labeled S6 and L9 ribosomal proteins, and reassembled 30S and 50S subunits to construct 70S ribosome particles.

\section{Construct design and rationale}

L7/L12 is unique in that the protein is present on the ribosome in four copies. L7/L12 constitutes a crucial part of the ribosomal L7/L12 stalk, believed to be involved in recruiting translation factors and subsequent GTPase activity. The N-terminal domain (NTD) of L7/L12 is involved in protein-protein interaction with L10, another ribosomal protein [53]; [54]. L10 anchors the tetramer and interfaces with rRNA on the 50S subunit. Structural analysis shows the C-terminal domain (CTD) is linked to the NTD via a flexible hinge region. The flexibility of this region is required for conformational changes during translation. Structural analysis of L12 shows a strictly conserved region on the CTD involved in binding to different translation factors. However, the extreme terminus of the CTD region was not found to be involved in any binding interactions and does not require the rotational freedom of the NTD. Thus, it is a good candidate for the insertion of an affinity tag for rapid purification. Indeed, researchers were able to 
incorporate a genomic His-tag in the L7/L12 gene ensuring that the produced ribosomes are homogeneously tetra $-(\mathrm{His})_{6}-$ tagged. Further, they showed, that the tetra-tag was highly specific and provided strong binding of ribosomes in cell lysate to a nickel affinity column [31].

To expedite both purification and fluorescent labeling of ribosomes, we decided to make genomic knockouts in the same strain containing a His-tag on the L7/L12 stalk. While any non-essential ribosomal protein can in principle be directed for labeling, we decided to target proteins S6 and L9 (Fig. 2, PDB ID:

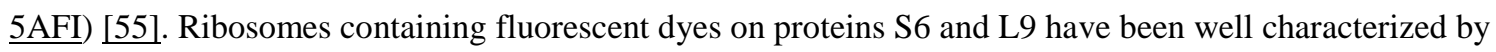
our studies and others to investigate intersubunit rotation and conformational dynamics [8]; [17]; [49]; [56]. The ribosome samples in these previous studies have been prepared by classical methods using partial and full reconstitution. However, in all cases 30S and 50S subunits were prepared separately and reassembled, which is known to result in reduced activity [17]; [20].

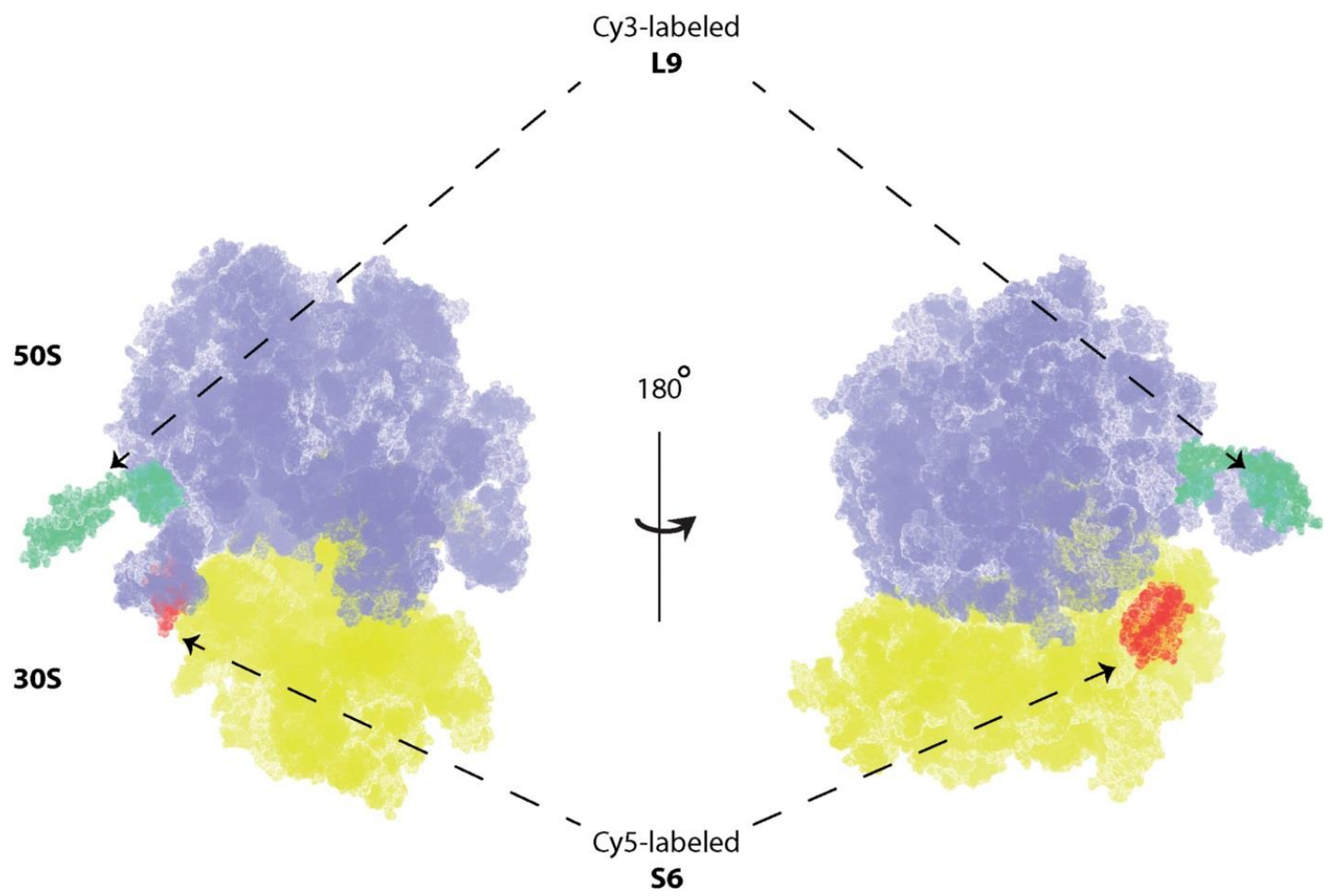

\subsubsection{Figure 26 Ribosome structure}

Figure 2: Ribosome structure highlighting L9 (green) and S6 (red). PDB ID: 5AFI. (For interpretation of the references to color in this figure legend, the reader is referred to the web version of this article.) Growth rate determination 
To measure the changes in growth rate as a result of the introduced genomic mutations, we grew all strains including controls in a temperature regulated plate reader as described in materials and methods. MRE600, which lacks ribonuclease I, is a commonly used E. coli strain for ribosome preparation, and BW25113 is the parent strain for the Keio collection. When compared to the parent strain BW25113, the L12 His-tagged strain (BD0012) showed a comparable growth rate consistent with previous results with

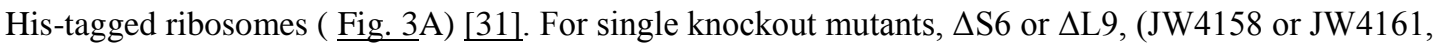
respectively) the growth rate was not significantly affected. However, the double knockout mutants (BD6900 or BD6912) required nearly double the time to reach the same OD as the parent strain. While non-essential for cell survival, the S6/L9 deletion in both strains could potentially have a negative effect on translation through reduced translation efficiency or ribosome assembly. Specific mutations or the complete absence of L9 is thought to interfere with the precise arrangement of the P-site tRNA [57]. S6 has no known function independent of binding directly to $16 \mathrm{~S}$ rRNA in the 30S subunit near the E-site [58]. However, it is clear that the cumulative effect of both deletions is not lethal. Since the stability and integrity of the ribosome is restored after reconstitution with S6 and L9 as shown below, the reason for reduced growth efficiency was not pursued further. The growth rate of all strains is not affected with the addition of antibiotics, kanamycin and chloramphenicol, to the cultures, which shows the stability of the deletion strains as evidenced by the maintenance of the antibiotic-resistance cassettes. (Fig. 3B). Finally, tagging the L7/L12 stalk with a tetra $-(\mathrm{His})_{6}-\operatorname{tag}$ did not affect the apparent growth rate of the WT strain. Also, there was no difference between the growth rate for BW6912 and BD6900, which only differ by a His-tag on the L7/L12 stalk.
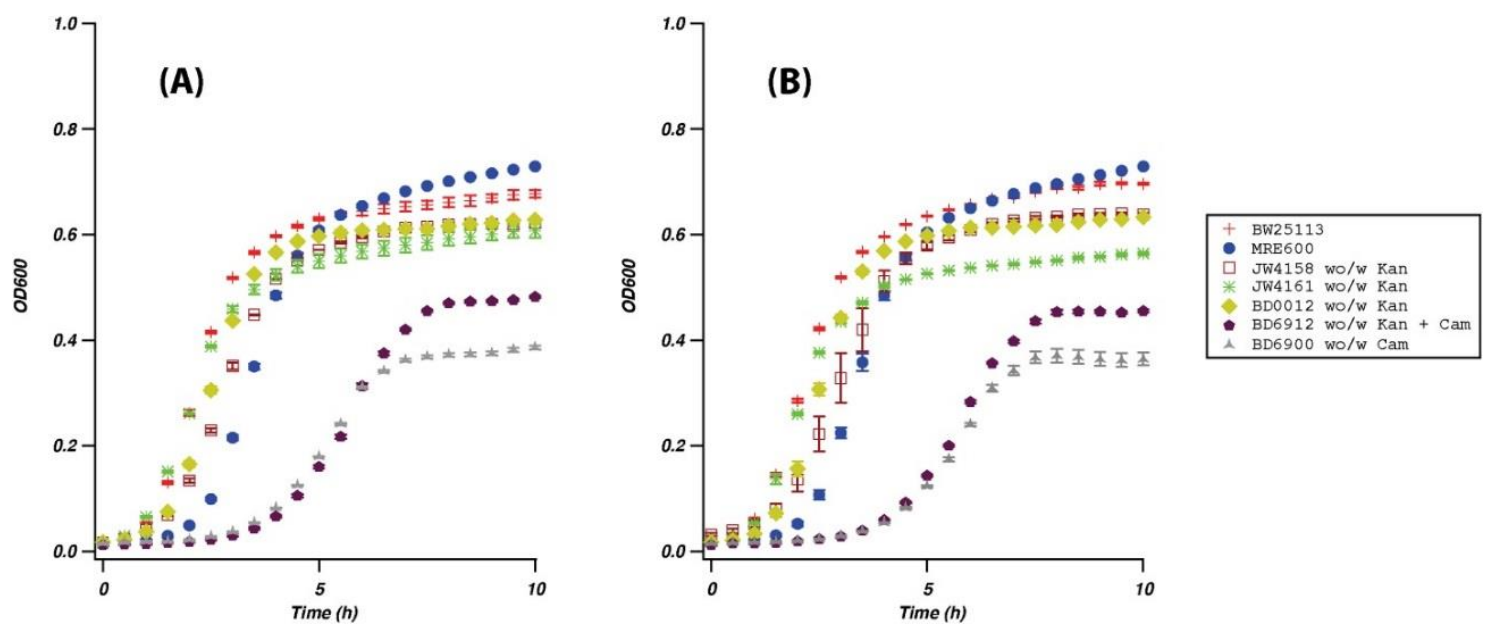


\subsubsection{Figure 27 Comparison of the growth rates}

Figure 3: Comparison of the growth rate of WT and mutant strains (Table 2) Without antibiotics (wo), and with antibiotics (w). Antibiotics used are kanamycin (Kan) and chloramphenicol (cam).

Sucrose gradient analysis

Similar to other reports, classically purified tight-coupled ribosomes under our purification conditions show a significant 50S peak along with a 70S peak (Fig. S1). The presence of 50S subunits indicates the dissociation of loosely bound subunits breaking up the 70S ribosome. In a classical subunit preparation, the magnesium concentration is lowered to dissociate the two subunits followed by a sucrose gradient purification to individually purify the two subunits [34]; [38]; [59]. Sucrose gradient peaks have to be collected conservatively to avoid cross-contamination, which has the negative consequence of obtaining a lower yield. As an example when purifying $70 \mathrm{~S}$ ribosomes as in Fig. S1, we selected only those fractions that would minimize $50 \mathrm{~S}$ contamination. This resulted in a loss of $\sim 10 \%$ of the total 70 S ribosomes present in the gradient. Additionally, $\sim 23 \%$ of $70 \mathrm{~S}$ ribosomes are lost due to dissociation into component subunits. To assess the purity of His-tagged WT ribosomes and subunits, we performed a sedimentation analysis using ultracentrifugation. These ribosomes or dissociated subunits were loaded on a 10-30\% analytical scale sucrose gradient. In contrast to tight-coupled classically purified ribosomes, His-tagged preparations, whether tight-coupled or subunit preparations, show primarily a single peak on the gradients (Fig. 4).

Hence, His-tagged ribosomes show tighter subunit coupling compared to a classical preparation.

Potentially, the constant exposure to elevated magnesium levels throughout the affinity-purification, reduced purification time, and the lack of ultracentrifugation preserves the integrity of the ribosomes in this context. Also, when performing a subunit preparation using His-tag purification, we were able to observe nearly complete dissociation of $50 \mathrm{~S}$ and $30 \mathrm{~S}$ subunits providing homogeneous samples of each subunit. Thus, affinity-purification preserves the integrity of ribosomes during purification and provides a superior method for separating dissociated subunits. 

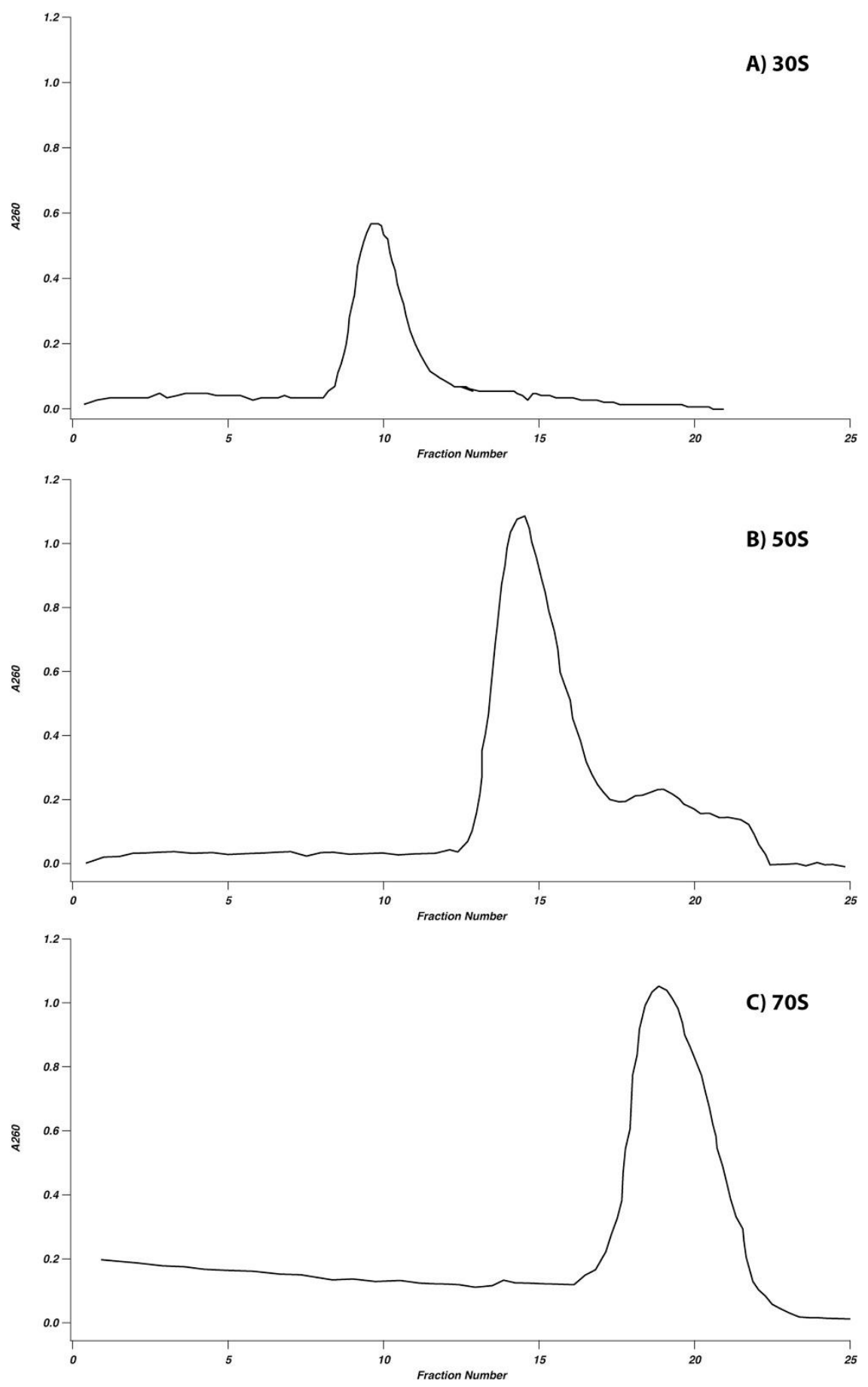

\subsubsection{Figure 28 Sucrose density-gradient analysis}

Figure 4: Sucrose density-gradient analysis of the His-tagged WT ribosomes and subunits. (A) 30S, (B) 50S, (C) 70S.

\section{D-gel analysis}


To compare the purity and consistency of the purified and/or reconstituted ribosomes, samples were denatured and subjected to SDS-PAGE (Fig. 5) [59]. S1 is the largest ribosomal protein and the only protein migrating above the $35 \mathrm{kDa}$ marker (Fig. 5A) [60]. The Coomassie-stained gel shows a comparable pattern among the four constructs, A through $\mathrm{D}$, with proteins grouping mostly in two major sets as shown previously [59]. When the gel was scanned for the presence of Cy3 and Cy5, fluorescent bands were present in the two center lanes, B and C, consistent with the molecular weights of Cy5-S6 and Cy3-L9 for both classically purified and His-tagged ribosomes (Fig. 5B). The protein-banding pattern is consistent across the four constructs and shows high purity with no crude cellular proteins. Further, this clearly shows that the fluorescently labeled proteins S6 and L9 were reconstituted into the purified knockout mutant ribosomes.

\section{A}
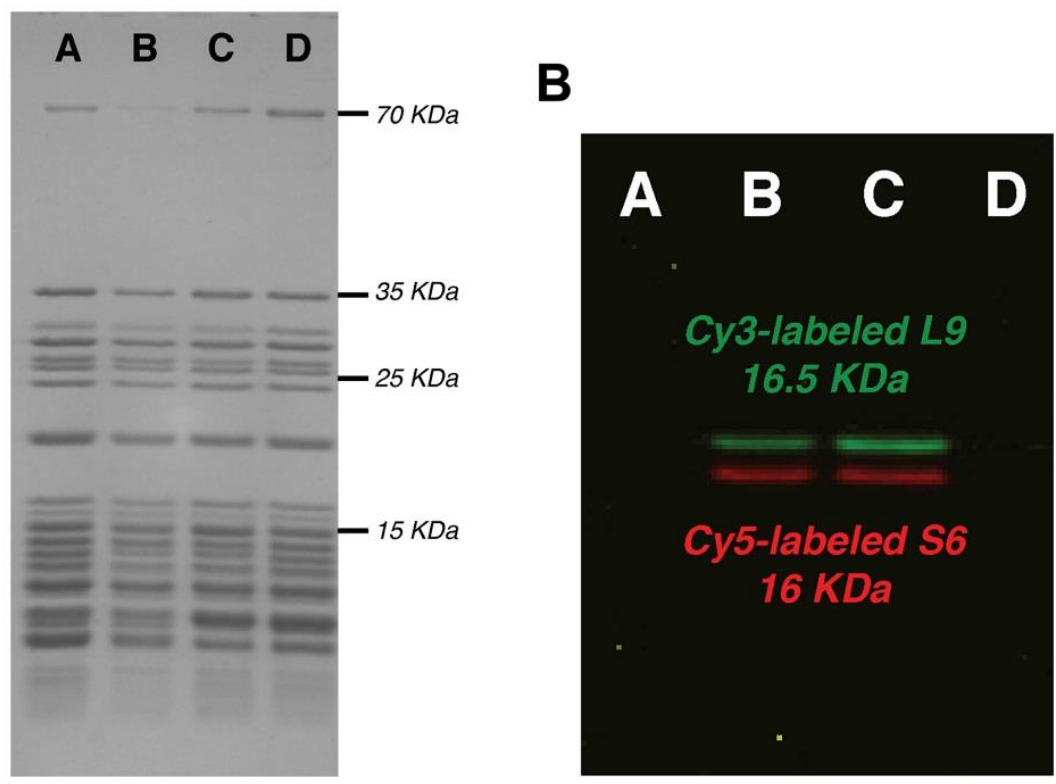

\subsubsection{Figure 29 SDS-PAGE of ribosomal constructs}

Figure 5: SDS-PAGE of ribosomal constructs. (A) Classically purified 70S WT ribosomes, (B) Classically purified 70S S6-Cy5/L9-Cy3 ribosomes, (C) His-tagged 70S S6-Cy5/L9-Cy3 ribosomes, (D) His-tagged 70S WT ribosomes. Left panel: Coomassie-staining with the size marker indicated. Right panel: overlapping channels for Cy3 and Cy5.

tRNA binding, PTC activity and EF-G translocation efficiency 
To assess tRNA binding efficiency and the integrity of both P and A sites, we employed radiolabeled N-Ac- $\left[{ }^{3} \mathrm{H}\right] \mathrm{Phe}_{\mathrm{TRNA}}{ }^{\text {Phe }}$ using filter binding as described in Materials and Methods. The results are expressed as a percentage of the efficiency/activity of the classically purified WT ribosomes (Fig. 6, Table S2). tRNA binding efficiency of His-tagged ribosomes is comparable for both $\mathrm{P}$ and A sites with respect to classically purified WT ribosomes [31]. Previously, S6/L9 ribosomes were constructed by full reconstitution of the $30 \mathrm{~S}$ and partial reconstitution of the $50 \mathrm{~S}$ with subsequent reassembly of the subunits to form $70 \mathrm{~S}$ ribosomes. It was reported that tRNA binding efficiency was $60 \%$ of WT ribosomes. In this report, we perform partial reconstitution for both S6 and L9 with either classically purified 30S and 50S subunits similar to more recent reports [22] or with intact 70S His-tagged ribosomes. We observed minimal differences within error in all cases between the fluorescently labeled ribosomes and WT classically or His-tag purified ribosomes. This suggests that the His-tagged fluorescently labeled ribosomes described here are identical within error to WT ribosomes in terms of tRNA binding efficiency.

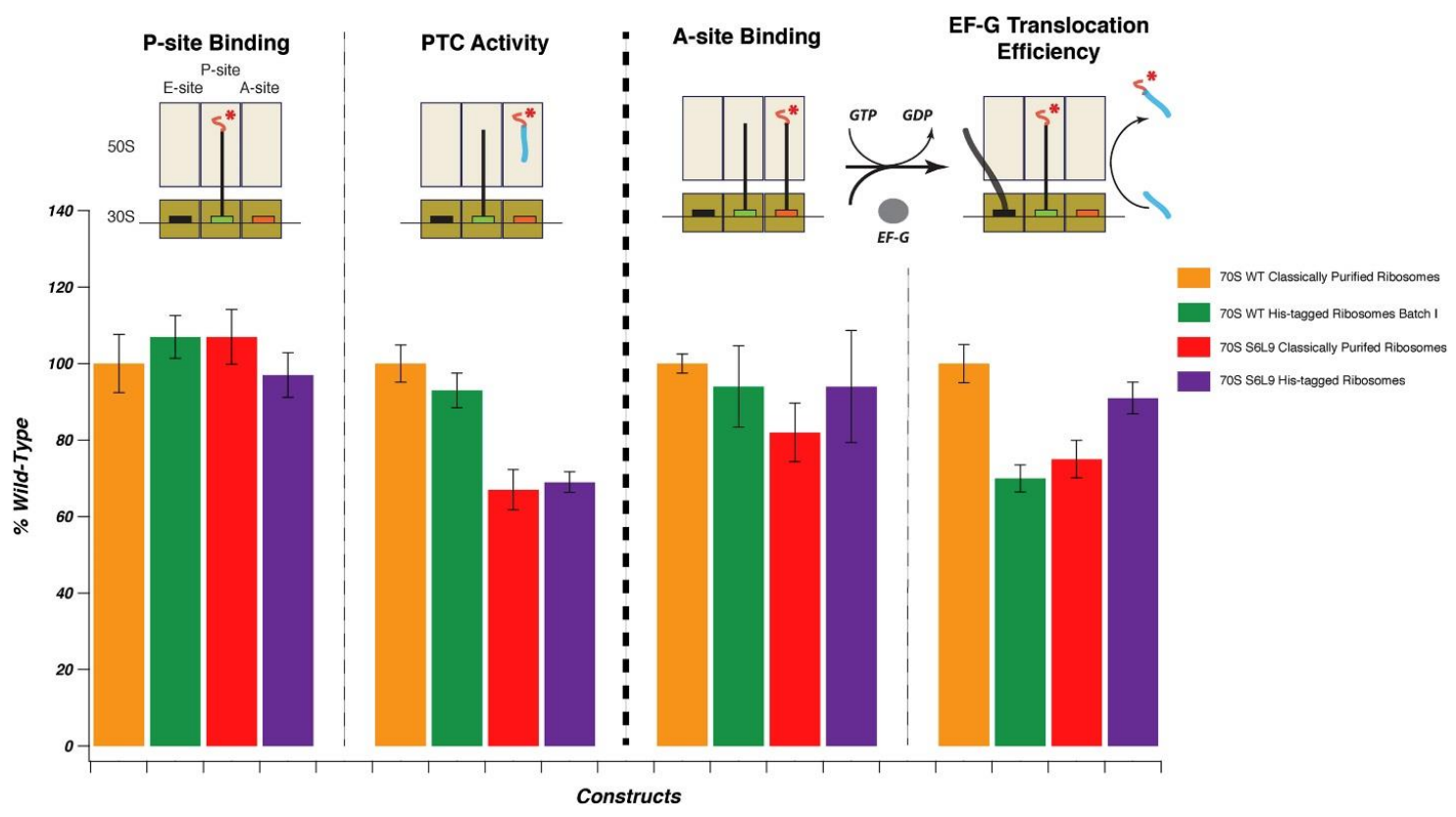

\subsubsection{Figure 30 tRNA binding}

Figure 6: tRNA binding, peptidyl transferase activity and translocation efficiency of WT and reconstituted ribosomes. Data is shown as a percentage of classically purified WT ribosomes. Cyan (puromycin), the asterisk indicates $[3 \mathrm{H}]$ Phe. (For interpretation of the references to color in this figure legend, the reader is referred to the web version of this article.) 
Next, ribosomes were tested for PTC activity by the addition of puromycin, which is an antibiotic that resembles a portion of the $3^{\prime}$ end of an aminoacyl tRNA [13]. Puromycin reacts preferentially with $P$ site aminoacyl tRNA over A site aminoacyl tRNA. Radiolabeled N-Ac- $\left[{ }^{3} H\right]$ Phe-tRNA ${ }^{\text {Phe }}$ was added to the P-site for each ribosomal constructs with subsequent addition of puromycin as described in Materials and Methods. The PTC activity was normalized against the P-site tRNA binding efficiency of the corresponding construct. When comparing PTC activity, we observe comparable activity between WT classically purified and His-tag purified ribosomes. In contrast to this, there was a drop to $\sim 70 \%$ activity for the S6/L9 labeled constructs. The reason for this is unclear, but S6 and/or L9 may have some unknown role in PTC activity.

Finally, translocation efficiency was verified by providing tRNA ${ }^{\text {Tyr }}$ to the P-site and radiolabeled N-Ac- $\left[{ }^{3} \mathrm{H}\right]$ Phe-tRNA ${ }^{\text {Phe }}$ to the A-site. EF-G.GTP was added as described and translocation efficiency was assessed by the addition of puromycin. In this case, all three constructs, WT His-tagged ribosomes, S6/L9 classically purified ribosomes, and S6/L9 His-tag purified ribosomes, exhibited reduced translocation efficiency as compared to WT classically purified ribosomes. However, the translocation efficiency of S6/L9 His-tag purified ribosomes showed the highest activity with translocation efficiency of $\sim 90 \%$. The apparent drop in translocation efficiency observed for the WT His-tagged ribosomes could result from batch-to-batch variability (see Supplementary Fig. S2). However, the data does not suggest that EF-G translocation efficiency drops due to the presence of a His-tag on the L7/L12 stalk as evidenced with the normal translocation efficiency shown for the His-tagged S6/L9 mutant and as shown previously [17]. Further, translocation is improved over previously reported reconstituted ribosomes [17]. smFRET measurements

To test fluorescently labeled S6 (Cy5)/L9 (Cy3) ribosome activity in smFRET experiments, Histagged and classically purified ribosomes were prepared in defined translation states as previously investigated [8]. The fluorescently labeled ribosomes were assembled at equilibrium conditions as described with a deacylated tRNA in the P-site, an amino acylated tRNA in the P-site or a deacylated tRNA in the P-site and an amino acylated tRNA in the A-site. The data were analyzed and traces exhibiting FRET were selected and combined into FRET histograms (Fig. 7). As observed previously, the histograms were 
best fit to two FRET states corresponding to a $\sim 0.4$ FRET rotated state and a $\sim 0.55$ FRET non-rotated state (Fig. 8). Based on the fitted histograms, we calculated the percentage of molecules residing in either state, rotated versus non-rotated. The acylation status of the P-site tRNA dictates the status of the majority of molecules [8]. Next, we calculated the equilibrium constant as the ratio between the rotated and non-rotated molecules. These data indicate that classically and His-tag purified ribosomes are identical and the results are consistent with previous observations ( $\underline{\text { Table } 3}$ ). Additionally, traces that transitioned between these two states were observed (Fig. 8). As before, the number of traces that fluctuate in a particular state is indicative of the dynamicity of the system. Again, the results are comparable between classical and His-tag purified ribosomes and previous reports. In general, the results are consistent with minor discrepancies between published and acquired data likely due to individual variations in data acquisition and sample preparation.

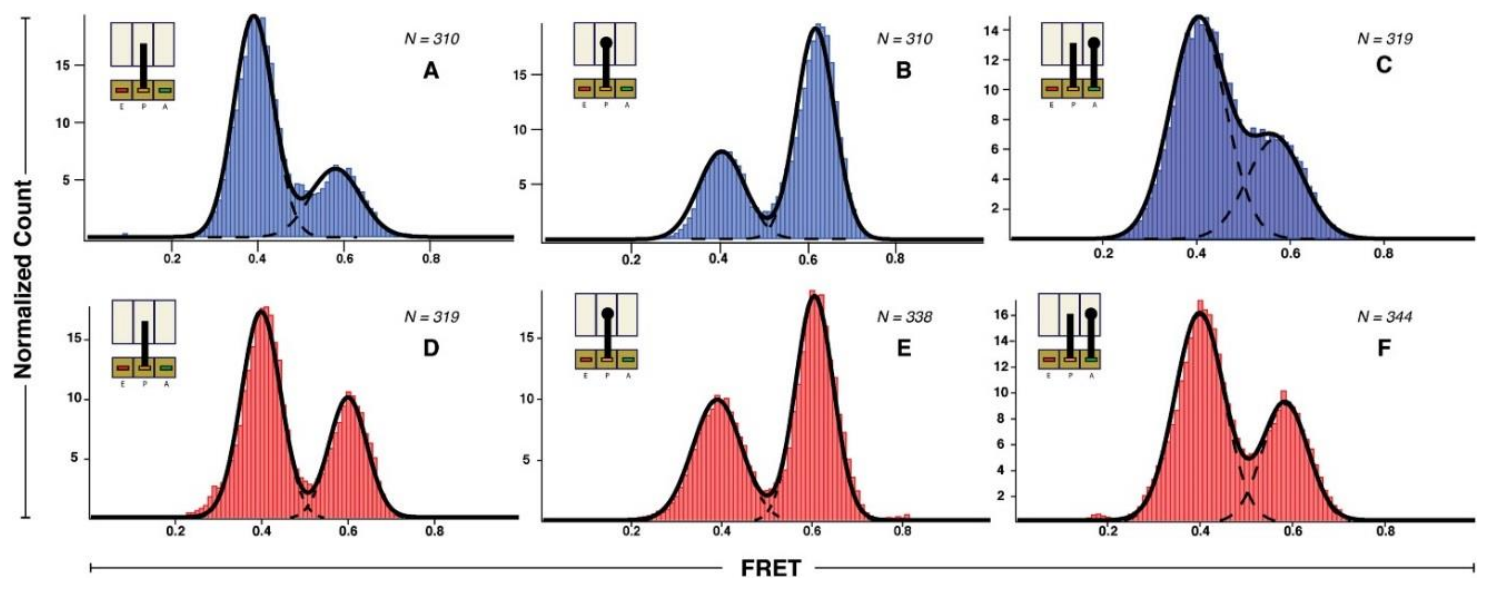

\subsubsection{Figure 31 Histograms of ribosome SmFRET data}

Figure 7: Histograms for different ribosomal complexes comparing classically purified ribosomes and Histagged ribosomes. Blue (classically purified S6L9 ribosomes) and red (His-tagged S6L9 ribosomes). (A) \& (D) $70 \mathrm{~S}$ ribosomes + mRNA + tRNAfMet in the P-site. (B) \& (E) 70S ribosomes + mRNA + fMettRNAfMet in the P-site. (C) \& (F) 70S ribosomes + mRNA + tRNAfMet in the P-site and NAc-PhetRNAPhe in the A-site. N: number of molecules. (For interpretation of the references to color in this figure legend, the reader is referred to the web version of this article.) 


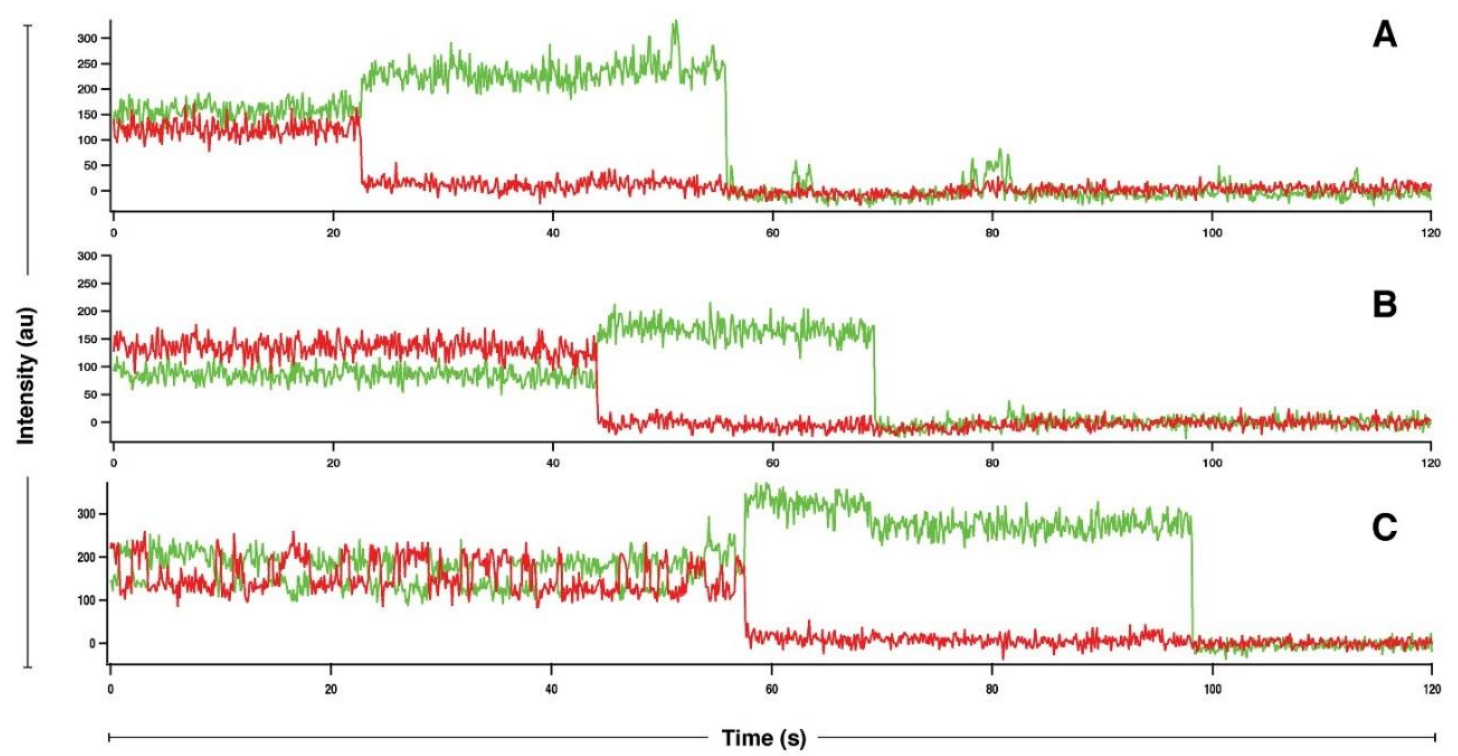

\subsubsection{Figure 32 Representative smFRET traces}

Figure 8: Representative smFRET traces for the reconstituted S6L9 ribosome mutant. Representative traces showing fluorescence intensities observed for the donor Cy3-lableled L9 (green) and the acceptor Cy5-labeled S6 (red) ribosome. A) 0.4 FRET - Rotated State, B) 0.6 FRET - non-rotated/Classical State, C) 0.4-0.6 Fluctuating trace. (For interpretation of the references to color in this figure legend, the reader is referred to the web version of this article.) 


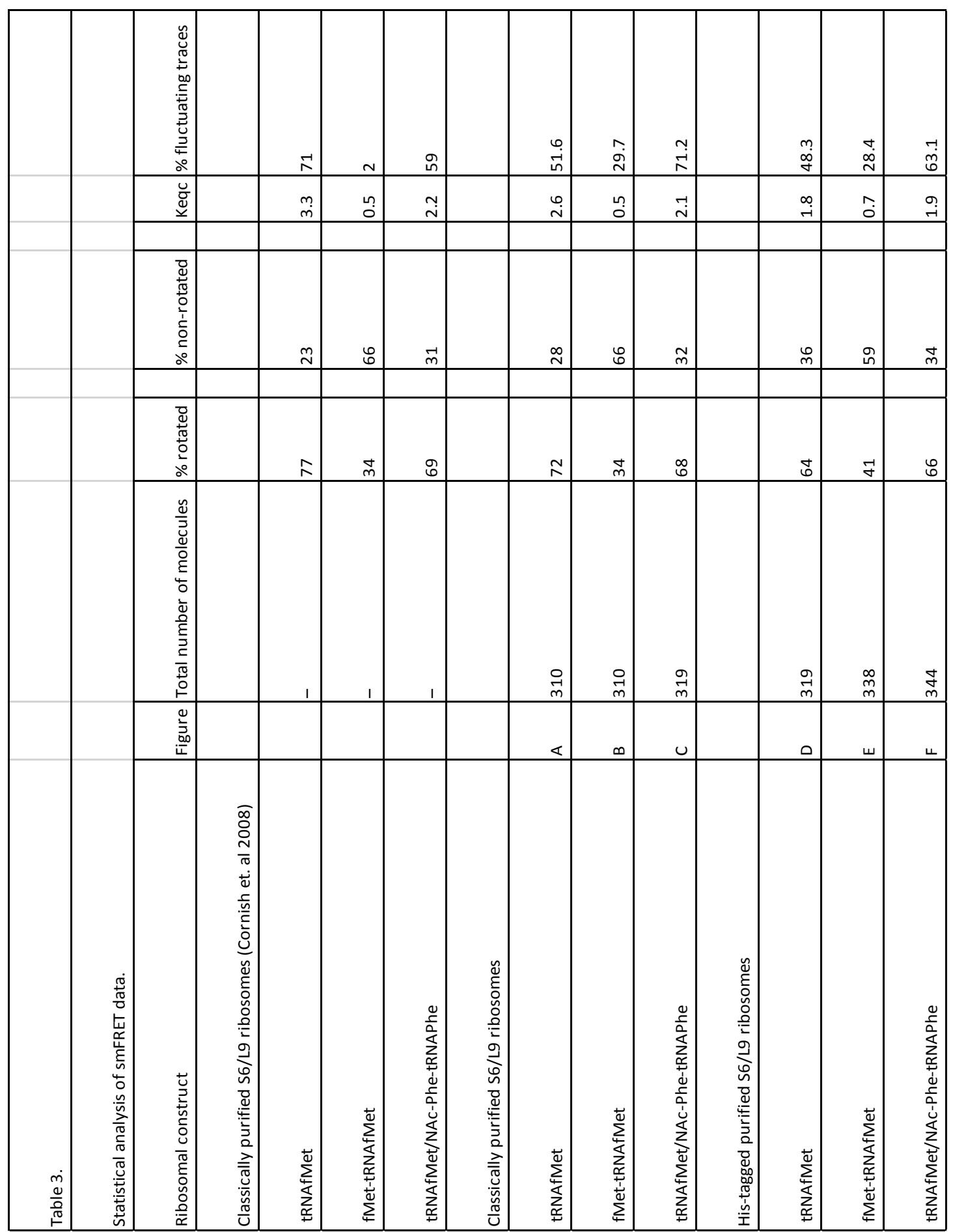

4.4.0 Table 5 Tabulated values

a Percentage of molecules in the rotated (0.4) state compared to the total number of molecules as calculated from the fitted histogram.

b Percentage of molecules in the non-rotated (0.6) state compared to the total number of molecules as calculated from the fitted histogram. 
c $\quad \mathrm{Keq}=[\%$ Rotated $] /[\%$ Non-Rotated $]$ as described previously.

d Percentage of the traces showing at least one transition event between rotated and non-rotated states compared to the total number of molecules as calculated from the fitted histogram.

\subsubsection{Conclusions}

Here, we present a faster, cheaper and simpler method of purifying and labeling ribosomes for fluorescence-based studies. The method presented makes it accessible to a wider audience of researchers as many researchers may lack the equipment for purifying ribosomes using classical methodology. In our experience, the preparation time is significantly reduced to as little as one day (as opposed to four days) with a sample yield of 2-3 times more than with the classical purification methods (Fig. 1). Also, when purifying ribosomes using the His-tag method, contamination of other subunits is significantly reduced or eliminated as compared to classical methods. Due to minimal sample manipulation and a gentler purification approach, the purified ribosomes are as good as, if not more active than, ribosomes purified by ultracentrifugation. Our studies and others have shown that antibiotics bound to the ribosome can significantly alter the conformational dynamics of the ribosome [8]; [61]; [62]. Thus, we envision that fluorescence based high-throughput screens could be a viable option for screening for new target molecules. The methodology developed here would provide the sample quantity and quality necessary for such experiments. In addition, using the His-tagged construct in conjunction with the labeling of ribosomal proteins S6 and L9, homogenous samples of different functional translation complexes produced from crude translation lysates could be easily pulled down and investigated with smFRET. It should be restated that in principle any non-essential ribosomal protein could be targeted for labeling for a fluorescent dye or any other molecule of interest. This would open new possibilities for purifying ribosome samples for contrast matching experiments in Small Angle Neutron Scattering (SANS), electron paramagnetic resonance (EPR) experiments, or even pursuing NMR based studies of individual ribosome subunits. Finally, the same principles could be applied to other cellular macromolecular complexes; facilitating the purification and labeling procedure.

\subsubsection{Supplementary information and supplementary tables}

\subsubsection{Table 6 Supplementary list of buffers used}

\section{Table S1. List of buffers used.}




\begin{tabular}{|c|c|}
\hline Buffer A & 20 mM Tris-HCl, $15.7 \mathrm{mM} \mathrm{MgCl} 2,100 \mathrm{mM}$ NH4Cl, 0.5 mM EDTA, $6 \mathrm{mM} \beta \mathrm{ME}$ \\
\hline Buffer B & $40 \mathrm{mM}$ Tris- $\mathrm{HCl}, 10 \mathrm{mM} \mathrm{MgCl} 2,100 \mathrm{mM} \mathrm{NH} 4 \mathrm{Cl}, 6 \mathrm{mM} \beta \mathrm{ME}$ \\
\hline Buffer C & $37.7 \%$ Sucrose, $20 \mathrm{mM}$ Tris- $\mathrm{HCl}, 15.7 \mathrm{mM} \mathrm{MgCl} 2,500 \mathrm{mM} \mathrm{NH} 4 \mathrm{Cl}, 0.5 \mathrm{mM}$ EDTA, $6 \mathrm{mM} \beta \mathrm{ME}$ \\
\hline Buffer D & $20 \mathrm{mM}$ Tris-HCl, $15.7 \mathrm{mM} \mathrm{MgCl} 2,100 \mathrm{mM} \mathrm{NH} 4 \mathrm{Cl}, 0.5 \mathrm{mM}$ EDTA, $6 \mathrm{mM} \beta \mathrm{ME}$ \\
\hline Buffer E & $37.7 \%$ Sucrose, $20 \mathrm{mM}$ Tris- $\mathrm{HCl}, 10.5 \mathrm{mM} \mathrm{MgCl} 2,500 \mathrm{mM} \mathrm{NH} 4 \mathrm{Cl}, 0.5 \mathrm{mM}$ EDTA, $6 \mathrm{mM} \beta \mathrm{ME}$ \\
\hline Buffer F & $50 \mathrm{mM}$ Tris- $\mathrm{HCl} \mathrm{pH} 7.0,1 \mathrm{mM} \mathrm{MgCl} 2,100 \mathrm{mM} \mathrm{NH} 4 \mathrm{Cl}, 6 \mathrm{mM} \beta \mathrm{ME}$ \\
\hline Buffer G & $20 \mathrm{mM}$ Tris- $\mathrm{HCl} \mathrm{pH} 7.6,10 \mathrm{mM} \mathrm{MgCl} 2,150 \mathrm{mM} \mathrm{KCl}, 30 \mathrm{mM} \mathrm{NH} 4 \mathrm{Cl}, 6 \mathrm{mM} \beta \mathrm{ME}$ \\
\hline Buffer H & $20 \mathrm{mM}$ Tris- $\mathrm{HCl} \mathrm{pH}$ 7.6, $10 \mathrm{mM} \mathrm{MgCl} 2,150 \mathrm{mM} \mathrm{KCl}, 30 \mathrm{mM} \mathrm{NH} 4 \mathrm{Cl}, 5 \mathrm{mM}$ Imidazole, $6 \mathrm{mM} \beta \mathrm{ME}$ \\
\hline Buffer I & $20 \mathrm{mM}$ Tris- $\mathrm{HCl} \mathrm{pH} 7.6,10 \mathrm{mM} \mathrm{MgCl} 2,150 \mathrm{mM} \mathrm{KCl}, 30 \mathrm{mM} \mathrm{NH} 4 \mathrm{Cl}, 150 \mathrm{mM}$ Imidazole, $6 \mathrm{mM} \beta \mathrm{ME}$ \\
\hline Buffer J & $20 \mathrm{mM}$ Tris- $\mathrm{HCl} \mathrm{pH} 7.6,1 \mathrm{mM} \mathrm{MgCl} 2,150 \mathrm{mM} \mathrm{KCl}, 30 \mathrm{mM} \mathrm{NH} 4 \mathrm{Cl}, 6 \mathrm{mM} \beta \mathrm{ME}$ \\
\hline Buffer K & $80 \mathrm{mM}$ HEPES-KOH pH 7.6, $20 \mathrm{mM} \mathrm{KCl,} 6 \mathrm{mM} \beta \mathrm{ME}$ \\
\hline Buffer L & Buffer L $20 \mathrm{mM} \mathrm{NaOAc}$ pH 5.6, $1 \mathrm{M} \mathrm{KCl}, 6 \mathrm{M}$ Urea, $6 \mathrm{mM} \beta \mathrm{ME}$ \\
\hline Buffer M & Buffer M $20 \mathrm{mM} \mathrm{NaOAc} \mathrm{pH} \mathrm{5.6,} 20 \mathrm{mM} \mathrm{KCl,} 6 \mathrm{M}$ Urea, $6 \mathrm{mM} \beta \mathrm{ME}$ \\
\hline Buffer N & Buffer N 30 mM HEPES-KOH pH 7.0, 20 mM MgCl2 1M KCl, 2x TCEP \\
\hline Buffer O & Buffer O $20 \mathrm{mM} \mathrm{NaOAc} \mathrm{pH} \mathrm{4.6,500} \mathrm{mM} \mathrm{KCL,} 6 \mathrm{M}$ Urea, $6 \mathrm{mM} \beta \mathrm{ME}$ \\
\hline Buffer P & Buffer P 20 mM NaOAc pH 4.6, 20 mM KCL 6 M Urea, 6 mM $\beta M E$ \\
\hline Buffer Q & Buffer Q $50 \mathrm{mM}$ Tris-HCl pH 7.0, $6 \mathrm{mM} \beta \mathrm{ME}$ \\
\hline Buffer R & Buffer R 30 mM HEPES-KOH pH 7.0, 4 mM MgCl2 1M NH4Cl, 2x TCEP \\
\hline Buffer S & Buffer S $80 \mathrm{mM}$ HEPES-KOH pH 7.6, $20 \mathrm{mM} \mathrm{MgCl} 2,330 \mathrm{mM} \mathrm{KCl,} 6 \mathrm{mM} \beta \mathrm{ME}$ and Nikkol $0.01 \% \mathrm{w} / \mathrm{v}$ \\
\hline Buffer T & Buffer T $50 \mathrm{mM}$ HEPES-KOH pH 7.5, $4 \mathrm{mM} \mathrm{MgCl} 2400 \mathrm{mM} \mathrm{NH} 4 \mathrm{Cl}, 6 \mathrm{mM} \beta \mathrm{ME}$ and Nikkol $0.01 \% \mathrm{w} / \mathrm{v}$ \\
\hline Buffer U & $\begin{array}{l}\text { Buffer U } 20 \mathrm{mM} \text { HEPES-KOH pH 7.5, } 20 \mathrm{mM} \mathrm{MgCl} 2,100 \mathrm{mM} \mathrm{NH} 4 \mathrm{Cl}, 6 \mathrm{mM} \beta \mathrm{ME} \text { and Nikkol } 0.01 \% \mathrm{w} / \mathrm{v}, 2 \\
\mathrm{mM} \text { Spermidine, } 0.1 \mathrm{mM} \text { Spermine }\end{array}$ \\
\hline Buffer V & Buffer V 50 mM Hepes-KOH (pH 7.6), 60 mM NH4Cl, 7 mM MgCl2, $6 \mathrm{mM} \beta \mathrm{ME}$ \\
\hline Buffer W & Buffer W 50 mM Hepes-KOH pH 7.6, 20 mM MgCl2, $100 \mathrm{mM} \mathrm{KCl,} \mathrm{0.01 \%} \mathrm{Nikkol,} 6 \mathrm{mM} \beta \mathrm{ME}$ \\
\hline Buffer X & Buffer X 50 mM Hepes pH 7.5, $20 \mathrm{mM} \mathrm{MgCl2,100} \mathrm{mM} \mathrm{NH4Cl,} 6 \mathrm{mM} \beta \mathrm{ME}$ \\
\hline Buffer Y & $\begin{array}{l}\text { Buffer Y } 20 \mathrm{mM} \text { HEPES-KOH pH 7.5, } 6 \mathrm{mM} \mathrm{MgCl} 2,150 \mathrm{mM} \mathrm{NH} 4 \mathrm{Cl}, 6 \mathrm{mM} \beta \mathrm{ME}, 2 \mathrm{mM} \text { spermidine, and } 0.1 \\
\mathrm{mM} \text { spermine }\end{array}$ \\
\hline
\end{tabular}




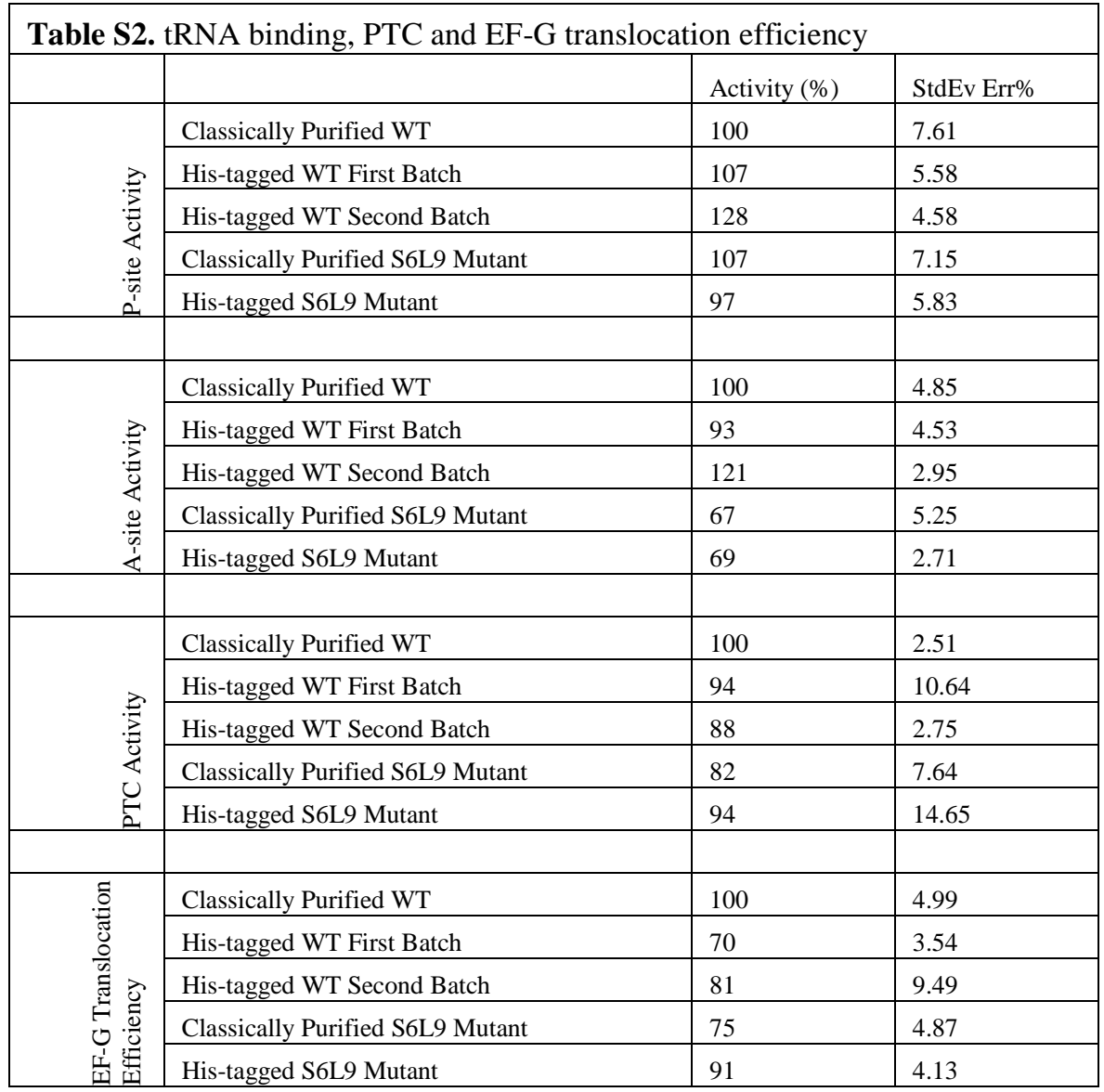

\subsubsection{Table 7 tRNA binding data}

\section{Supplementary Figures}

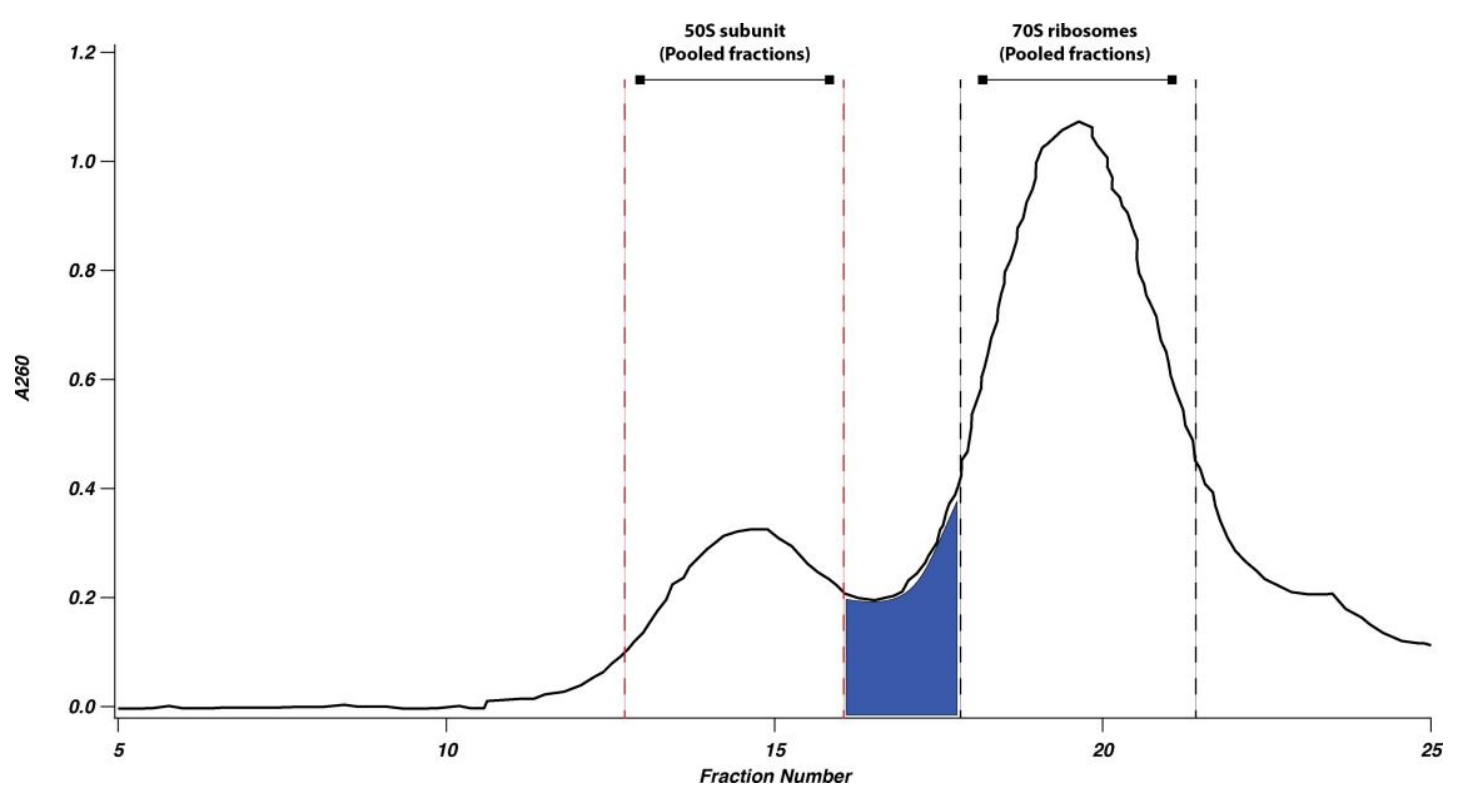

4.6.0 Figure 33 Sucrose density-gradient analysis 
Figure Supplementary 1. Sucrose density-gradient analysis of the classically purified WT ribosomes and subunits. The overlapping fractions are highlighted in cyan.

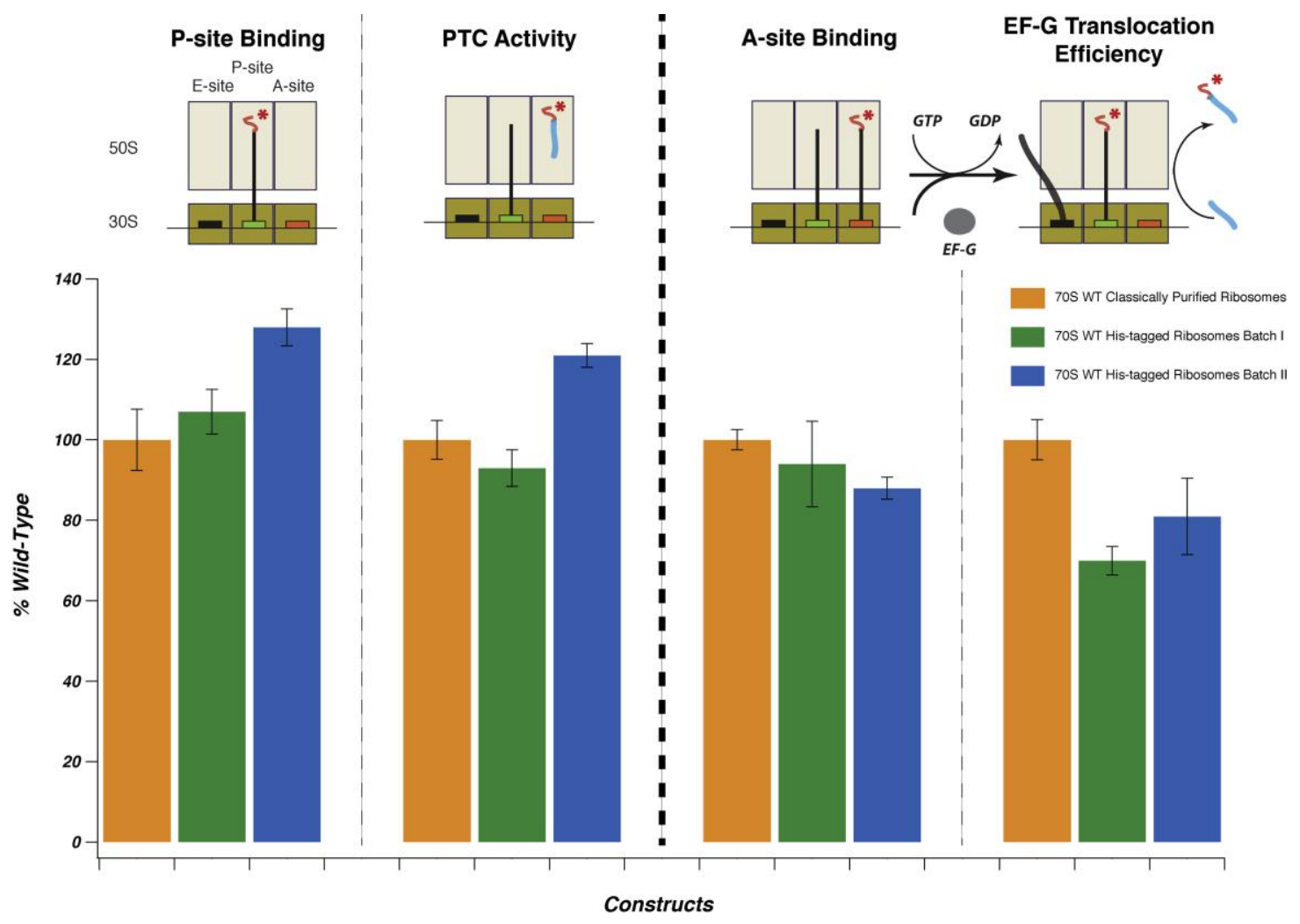

\subsubsection{Figure 34 tRNA binding}

Figure Supplementary 2. tRNA binding, peptidyl transferase activity and translocation efficiency of different batches of WT ribosomes. Data is shown as a percentage of classically purified WT ribosomes. Cyan (puromycin), the asterisk indicates $[3 \mathrm{H}]$ Phe.

\section{Supplementary Methods}

\section{Classical purification of tight-coupled ribosomes}

All ultracentrifugation steps performed in which a rotor is not specifically indicated were performed using a Type $60 \mathrm{Ti}$ or Type $45 \mathrm{Ti}$ fixed angle rotor. For all buffer composition refer to table S1. A fresh overnight culture of E. coli BW21153, wild-type (WT) or mutant strains, was used to inoculate an LB culture. The culture was grown with shaking at $37^{\circ} \mathrm{C}$ until an OD600 of 0.6. The culture was left to cool on ice with occasional shaking. Cells were harvested by centrifugation in a Sorvall SLC-6000 at 3,000 RPM for 20 min. The cell-pellet was briefly resuspended in cold buffer A, spun down and stored at $-80^{\circ} \mathrm{C}$. The cell-pellet was resuspended in buffer B and lysed using a French Press. The cell debris was spun down 
twice in a Sorvall SS-34 at 12,000 RPM for 20 min at $4^{\circ} \mathrm{C}$. The clarified supernatant was layered onto a $37.7 \%$ sucrose cushion in buffer $\mathrm{C}$. The ribosomes were pelleted by ultracentrifugation at $100,000 \mathrm{x} \mathrm{g}$ for $21 \mathrm{~h}$ at $4^{\circ} \mathrm{C}$. The ribosome pellet was rinsed carefully and resuspended in buffer D. The $\mathrm{NH} 4 \mathrm{Cl}$ concentration was adjusted to $400 \mathrm{mM}$ and pelleted by ultracentrifugation at $100,000 \mathrm{xg}$ for $4.5 \mathrm{~h}$ at $4{ }^{\circ} \mathrm{C}$. The pelleted ribosomes were rinsed and resuspended in buffer B. $10 \mathrm{mg}$ of ribosomes were layered on top of a $10-35 \%$ sucrose gradient in an SW28 rotor and ultracentrifuged at 53,000 x $\mathrm{g}$ for $13 \mathrm{~h}$ at $4^{\circ} \mathrm{C}$. The gradients were pumped using $40 \%$ sucrose using a Brandel gradient fractionator. The 70 S ribosome peak was conservatively pooled and the final $\mathrm{MgCl} 2$ concentration was adjusted to $10 \mathrm{mM}$. Purified ribosomes were pelleted by ultracentrifugation at $100,000 \mathrm{x} \mathrm{g}$ for $17 \mathrm{~h}$ at $4{ }^{\circ} \mathrm{C}$. The pelleted ribosomes were resuspended and stored in buffer $\mathrm{B}$ at $-80^{\circ} \mathrm{C}$. An alternative to ultracentrifugation for concentrating ribosomes was to use a Millipore Centricon® with a molecular weight cutoff of $100 \mathrm{~K}$ for concentration and buffer exchange before freezing and storage.

\section{Classical purification of $50 \mathrm{~S}$ and $30 \mathrm{~S}$ subunits}

Purification of 50S and 30S subunits were performed following a similar methodology as mentioned above. In brief, cell-pellets were lysed by French Press and clarified by centrifugation. The clarified supernatant was layered onto a $37.7 \%$ sucrose cushion in buffer E and pelleted by ultracentrifugation at $150,000 \mathrm{x}$ f for $16 \mathrm{~h}$ at $4^{\circ} \mathrm{C}$. The pellet was resuspended and dialyzed $3 \mathrm{x}$ against $1 \mathrm{~L}$ of buffer $\mathrm{F}$ for $1 \mathrm{~h}$ each at $4^{\circ} \mathrm{C} .10-35 \mathrm{mg}$ of ribosomes were layered on top of a $10-30 \%$ sucrose gradient and centrifuged in an SW28 rotor at $83,000 \mathrm{x}$ f for $14 \mathrm{~h}$ at $4^{\circ} \mathrm{C}$. The sucrose gradients were pumped using $40 \%$ sucrose using a Brandel gradient fractionator and appropriate fractions were collected. The $\mathrm{MgCl} 2$ concentration was adjusted to $10 \mathrm{mM}$ and the ribosome subunits were pelleted by ultracentrifugation at $80,000 \mathrm{x}$ g for 21 at $4^{\circ} \mathrm{C}$. Pelleted subunits were rinsed, resuspended and stored at $-80^{\circ} \mathrm{C} . \Delta \mathrm{S} 630 \mathrm{~S}$ was resuspended in buffer S, $\Delta \mathrm{L} 950 \mathrm{~S}$ was resuspended in buffer T and WT 30S and WT 50S were resuspended in buffer B.

\subsubsection{Acknowledgments}

The authors thank Bob Sauer for providing the P1 Phage. This work was supported by National Science Foundation CAREER award [grant number: MCB-115343] to P.V.C. P.V.C is a Pew Scholar in the Biomedical Sciences. Funding for open access charge: National Science Foundation. 


\subsubsection{Contributions}

Drew Menke conceived the project, helped with the genomic manipulation, was responsible for the generation of all of the constructs, performed smFRET experimentation on the classically made S6/L9 sample, analyzed the smFRET data, aided in the tRNA binding experiments and helped write the initial manuscript.

\subsubsection{References}

1 Korostelev, A., D.N. Ermolenko, and H.F. Noller, Structural dynamics of the ribosome. Current opinion in chemical biology, 2008. 12(6): p. 674-683.

2 Frank, J. and R.K. Agrawal, A ratchet-like inter-subunit reorganization of the ribosome during translocation. Nature, 2000. 406(6793): p. 318-22.

3 Wimberly, B.T., et al., Structure of the 30S ribosomal subunit. Nature, 2000. 407(6802): p. 327-39.

4 Ban, N., et al., The complete atomic structure of the large ribosomal subunit at 2.4 A resolution. Science (New York, N.Y.), 2000. 289(5481): p. 905-920.

5 Yusupov, M.M., et al., Crystal structure of the ribosome at 5.5 A resolution. Science (New York, N.Y.), 2001. 292(5518): p. 883-896.

6 Shebl, B., Z. Norman, and P.V. Cornish, Ribosome structure and dynamics by smFRET microscopy. Methods Enzymol, 2014. 549: p. 375-406.

7 Cornish, P.V., et al., Spontaneous intersubunit rotation in single ribosomes. Mol Cell, 2008. 30(5): p. $578-88$.

8 Blanchard, S.C., et al., tRNA dynamics on the ribosome during translation. Proc Natl Acad Sci U S A, 2004. 101(35): p. 12893-8.

9 Cornish, P.V., et al., Following movement of the L1 stalk between three functional states in single ribosomes. Proc Natl Acad Sci U S A, 2009. 106(8): p. 2571-6.

10 Fei, J., et al., Coupling of Ribosomal L1 Stalk and tRNA Dynamics during Translation Elongation. 2008. 30: p. 348-359.

11 Chen, C., et al., Dynamics of translation by single ribosomes through mRNA secondary structures. Nat Struct Mol Biol, 2013. 20(5): p. 582-8. 
12 Munro, J.B., et al., Identification of two distinct hybrid state intermediates on the ribosome. Mol Cell, 2007. 25(4): p. 505-17.

13 Moazed, D. and H.F. Noller, Intermediate states in the movement of transfer RNA in the ribosome. Nature, 1989. 342(6246): p. 142-148.

14 Munro, J.B., et al., A fast dynamic mode of the EF-G-bound ribosome. EMBO J, 2010. 29(4): p. 77081.

15 Salsi, E., et al., Following movement of domain IV of elongation factor G during ribosomal translocation. Proceedings of the National Academy of Sciences of the United States of America, 2014. 111(42): p. 15060-15065.

16 Dorywalska, M., et al., Site-specific labeling of the ribosome for single-molecule spectroscopy. Nucleic Acids Res, 2005. 33(1): p. 182-9.

17 Ermolenko, D.N., et al., Observation of intersubunit movement of the ribosome in solution using FRET. Journal of molecular biology, 2007. 370(3): p. 530-540.

18 Ermolenko, D.N., et al., Antibiotics that bind to the A site of the large ribosomal subunit can induce mRNA translocation. RNA, 2013. 19(2): p. 158-66.

19 Marshall, R.A., M. Dorywalska, and J.D. Puglisi, Irreversible chemical steps control intersubunit dynamics during translation. Proceedings of the National Academy of Sciences of the United States of America, 2008. 105(40): p. 15364-15369.

20 Culver, G.M. and H.F. Noller, [30] In vitro reconstitution of 30s ribosomal subunits using complete set of recombinant proteins. 2000. 446.

21 Svidritskiy, E., et al., Blasticidin S inhibits translation by trapping deformed tRNA on the ribosome. Proc Natl Acad Sci U S A, 2013. 110(30): p. 12283-8.

22 Hickerson, R., et al., Measurement of internal movements within the $30 \mathrm{~S}$ ribosomal subunit using Förster resonance energy transfer. Journal of molecular biology, 2005. 354(2): p. 459-472.

23 Staehelin, T. and D.R. Maglott, [47] Preparation of Escherchia coli ribosomal subunits active in polypeptide synthesis. 1971. 449. 
24 Culver, G.M. and H.F. Noller, Efficient reconstitution of functional Escherichia coli 30S ribosomal subunits from a complete set of recombinant small subunit ribosomal proteins. RNA (New York, N.Y.), 1999. 5(6): p. 832-843.

25 Marshall, R.A., et al., Translation at the single-molecule level. Annual review of biochemistry, 2008. 77: p. 177-203.

26 Roy, R., S. Hohng, and T. Ha, A practical guide to single-molecule FRET. Nature methods, 2008. 5(6): p. 507-516.

27 Perez, C.E. and R.L. Gonzalez, In vitro and in vivo single-molecule fluorescence imaging of ribosome-catalyzed protein synthesis. Current opinion in chemical biology, 2011. 15(6): p. 853-863.

28 Jelenc, P.C., Rapid purification of highly active ribosomes from Escherichia coli. Analytical Biochemistry, 1980. 105(1): p. 369.

29 Kopaskie, K.S., K.G. Ligtenberg, and O. Schneewind, Translational regulation of Yersinia enterocolitica mRNA encoding a type III secretion substrate. The Journal of biological chemistry, 2013. 288(49): p. 35478-35488.

30 Gan, X., et al., Tag-mediated isolation of yeast mitochondrial ribosome and mass spectrometric identification of its new components. European Journal of Biochemistry, 2002. 269(21): p. 5203.

31 Inada, T., et al., One-step affinity purification of the yeast ribosome and its associated proteins and mRNAs. RNA (New York, N.Y.), 2002. 8(7): p. 948-958.

32 Leonov, A.A., et al., Affinity purification of ribosomes with a lethal G2655C mutation in 23S rRNA that affects the translocation. The Journal of biological chemistry, 2003. 278(28):p. 25664-25670.

33 Youngman, E.M. and R. Green, Affinity purification of in vivo-assembled ribosomes for in vitro biochemical analysis. Methods (San Diego, Calif.), 2005. 36(3): p. 305-312.

34 Zanetti, M.E., et al., Immunopurification of polyribosomal complexes of Arabidopsis for global analysis of gene expression. Plant physiology, 2005. 138(2): p. 624-635.

35 Ederth, J., et al., A single-step method for purification of active His-tagged ribosomes from a genetically engineered Escherichia coli. Nucleic Acids Research, 2008. 37(2): p.e15.

36 Wang, L., et al., Allosteric control of the ribosome by small-molecule antibiotics. Nat Struct Mol Biol, 2012. 19(9): p. 957-63. 
37 Wasserman, M.R., et al., Chemically related 4,5-linked aminoglycoside antibiotics drive subunit rotation in opposite directions. Nat Commun, 2015. 6: p. 7896.

38 Datsenko, K.A. and B.L. Wanner, One-step inactivation of chromosomal genes in Escherichia coli K12 using PCR products. Proceedings of the National Academy of Sciences of the United States of America, 2000. 97(12): p. 6640-6645.

39 Fei, J., et al., A Highly Purified, Fluorescently Labeled In Vitro Translation System for SingleMolecule Studies of Protein Synthesis. 2010. 472: p. 221-259.

40 Walker, S.E. and K. Fredrick, Preparation and evaluation of acylated tRNAs. Methods, 2008. 44(2): p. 81-6.

41 Dubnoff, J.S. and U. Maitra, Isolation and properties of polypeptide chain initiation factor FII from Escherichia coli: evidence for a dual function. Proceedings of the National Academy of Sciences of the United States of America, 1971. 68(2): p. 318-323.

42 Takyar, S., R.P. Hickerson, and H.F. Noller, mRNA helicase activity of the ribosome. Cell, 2005. 120(1): p. 49-58.

43 Milligan, J.F., et al., Oligoribonucleotide synthesis using T7 RNA polymerase and synthetic DNA templates. Nucleic Acids Research, 1987. 15(21): p. 8783.

44 Fredrick, K. and H.F. Noller, Accurate translocation of mRNA by the ribosome requires a peptidyl group or its analog on the tRNA moving into the 30S P site. Molecular Cell, 2002.9(5): p. 1125-1131.

45 Wilson, K.S. and H.F. Noller, Mapping the position of translational elongation factor EF-G in the ribosome by directed hydroxyl radical probing. Cell, 1998. 92(1): p. 131-9.

46 Spiegel, P.C., D.N. Ermolenko, and H.F. Noller, Elongation factor G stabilizes the hybridstate conformation of the 70S ribosome. RNA (New York, N.Y.), 2007. 13(9): p. 1473-1482.

47 Blaha, G., et al., [19] Preparation of functional ribosomal complexes and effect of buffer conditions on tRNA positions observed by cryoelectron microscopy. 2000. 292.

48 Umekage, S. and T. Ueda, Spermidine inhibits transient and stable ribosome subunit dissociation. FEBS Lett, 2006. 580(5): p. 1222-6.

49 Qin, P., et al., Structured mRNA induces the ribosome into a hyper-rotated state. EMBO Rep, 2014. 15(2): p. 185-90. 
50 Joo, C. and T. Ha, Labeling DNA (or RNA) for single-molecule FRET. Cold Spring Harbor protocols, 2012. 2012(9): p. 1005-1008.

51 Joo, C. and T. Ha, Single-molecule FRET with total internal reflection microscopy. Cold Spring Harbor protocols, 2012. 2012(12): p. pdb.top072058.

52 Elvekrog, M.M. and R.L. Gonzalez, Jr., Conformational selection of translation initiation factor 3 signals proper substrate selection. Nat Struct Mol Biol, 2013. 20(5): p. 628-33.

53 Diaconu, M., et al., Structural basis for the function of the ribosomal L7/12 stalk in factor binding and GTPase activation. Cell, 2005. 121(7): p. 991-1004.

54 Helgstrand, M., et al., The ribosomal stalk binds to translation factors IF2, EF-Tu, EF-G and RF3 via a conserved region of the L12 C-terminal domain. J Mol Biol, 2007. 365(2):p. 468-79.

55 Fischer, N., et al., Structure of the E. coli ribosome-EF-Tu complex at $<3$ A resolution by Cscorrected cryo-EM. Nature, 2015. 520(7548): p. 567-70.

56 Ling, C. and D.N. Ermolenko, Initiation factor 2 stabilizes the ribosome in a semirotated conformation. Proc Natl Acad Sci U S A, 2015. 112(52): p. 15874-9.

57 Adamski, F.M., J.F. Atkins, and R.F. Gesteland, Ribosomal protein L9 interactions with 23 S rRNA: the use of a translational bypass assay to study the effect of amino acid substitutions. J Mol Biol, 1996. 261(3): p. 357-71.

58 Brodersen, D.E., et al., Crystal structure of the $30 \mathrm{~S}$ ribosomal subunit from Thermus thermophilus: structure of the proteins and their interactions with 16 S RNA. J Mol Biol, 2002. 316(3): p. 725-68.

59 Mehta, P., et al., Ribosome purification approaches for studying interactions of regulatory proteins and RNAs with the ribosome. Methods Mol Biol, 2012. 905: p. 273-89.

60 Schnier, J., et al., Primary structure of Escherichia coli ribosomal protein S1 and of its gene rpsA. Proc Natl Acad Sci U S A, 1982. 79(4): p. 1008-11.

61 Ermolenko, D.N., et al., The antibiotic viomycin traps the ribosome in an intermediate state of translocation. Nature structural \& molecular biology, 2007. 14(6): p. 493-497.

62 Llano-Sotelo, B., et al., Fluorescently labeled ribosomes as a tool for analyzing antibiotic binding. RNA, 2009. 15(8): p. 1597-604. 


\subsubsection{Chapter 5: Using smFRET to dissect the L1 stalk-tRNA interaction}

Menke, D. E., Shebl, B., \& Cornish, P. V. 


\subsubsection{Abstract}

The ribosome undergoes large-scale dynamic rearrangement when synthesizing proteins during translation. Throughout translation, the ribosome interacts with various initiation, elongation, and terminations factors, as well as various other components that help to regulate and facilitate protein synthesis. These interactions result in large-scale structural rearrangements which are responsible for the preferential stabilization and shift in the distribution of states that influences how the ribosome interacts and responds. Despite continued research on translation, a number of important questions still remain unanswered as to the coordination of large-scale dynamics and interconnectivity of the ribosome.

Here, we use smFRET to investigate two large-scale dynamic motions of the ribosome, subunit rotation and L1 stalk motion, by disrupting key interactions between the L1 stalk and tRNA or removal of the L1 protein component of the L1 stalk. Our findings demonstrate that disruption of key interactions at the L1 stalk-tRNA interfaces, results in a reduction in the ribosome's ability to stabilize the L1 stalk in the closed state. Furthermore, our investigations of the construct lacking the ribosomal protein component of the L1 stalk resulted in the ribosome unable to stabilize in the rotated state.

Our smFRET data supports the hypothesis that the L1 stalk-tRNA interaction plays a critical role in both the local motion of the L1 stalk but also global dynamics of subunit rotation.

\subsubsection{Introduction}

The Escherichia coli ribosome is a macromolecular complex made up of two subunits, the $30 \mathrm{~S}$, which contains $\sim 21$ proteins and the $16 \mathrm{~S}$ rRNA, and the 50S, which consist of the $23 \mathrm{~S}$ and 5S rRNA and $\sim 34$ proteins. The ribosome is responsible for the synthesis of protein during translation. Translation is ubiquitous throughout all known organisms, and, without it, life would not be possible. Large-scale dynamics have been shown to be an integral part of the mechanism 
by which the ribosome synthesizes protein. The importance of the ribosome to the cell makes it an excellent target for the development and application of drugs.

FRET is the non-radiative transfer of energy from a donor fluorophore to an acceptor fluorophore within a defined distance, typically within 2-10 nanometers. Single molecule fluorescence resonance energy transfer (SmFRET), an adaptation of FRET, allows for the observation of a single molecule and reports on distance changes that can be used to examine the real time dynamics of a biomolecule. Constructs are specifically engineered to contain a donor and acceptor fluorophore at defined positions on the molecule in order to examine a specific process and design is often aided by structural data from X-ray crystallography and cryoEM.

For the past decade, smFRET has been used to study the dynamics of numerous systems including the ribosome. SmFRET data has shown that dynamics play a fundamental role in the mechanism of the ribosome during translation. The major dynamic motions of the ribosome have been studied extensively and have given the field a better understanding of how the ribosome functions during translation. When first investigating the ribosome with smFRET, studies examined a single motion to try to understand its importance and contribution to the ribosome's function.

Inter-subunit rotation is the rotation of the $30 \mathrm{~S}$ subunit counterclockwise and $50 \mathrm{~S}$ in the clockwise, when viewed from the top, and is the largest dynamic motion of the ribosome. During translation under equilibrium conditions, the rotation state of the ribosome exists as a distribution of states, and the ribosome is able to freely fluctuate between the rotated and non-rotated states. The rotation state of the ribosome is affected by the type of tRNA in the A, P and E site of the ribosome, the acylation status of the tRNA bound to the ribosome, and other factors involved [1]. When the ribosomes are unable to rotate, the ribosomes becomes inactive and unable to synthesize protein, and thus ribosome rotation is required for translation [2].

The L1 stalk is made up of a portion of the 23S, rRNA, including helix 76, 77 and 78, as well as the L1 stalk protein and has been shown to be involved in the removal of the tRNA from 
the E site of the ribosome as well as to make contact with tRNA within the ribosome during translocation $[3,17]$. Three distinct states have been identified for the L1 stalk, the open, half closed, and closed states, and their distribution is determined by the tRNA bound to the A, P and E site of the ribosome $[4,5]$.

The motion of tRNA within the ribosome during elongation can form a number of states involved in the binding and accommodation of tRNA onto the ribosome [6]. Typically, the tRNA within the ribosomes occupy the A/A and P/P sites of the ribosome, which correspond to the $30 \mathrm{~S}$ and $50 \mathrm{~S}$ portion of the ribosome. Further studies have identified additional states corresponding to what have been termed the hybrid states, which is when the tRNA occupies different sites of both the $30 \mathrm{~S}$ and 50S and is split between, A/P and P/E sites [7]. The specific tRNA in the A, P and $\mathrm{E}$ site of the ribosome, the acylation status of the tRNA bound to the ribosome, and any additional factors involved can influence the structure of the rest of the ribosome. Additional evidence points to positioning, and thermal fluctuations that make up the primary mechanism for initial tRNA selection [8]. The environment of the ribosome can have a large effect on its dynamics, variation in the $\mathrm{MgCl}_{2}$ concentration has been shown to bias the distribution of states for tRNA bound to the ribosome when observed by smFRET [9]. At times, solution conditions and their relevancy to environmental conditions has been a challenge of smFRET studies.

It is possible to disrupt key interactions of the ribosome by mutational analysis. Previous studies using mutant tRNA combined with smFRET experiments have provided insight into the type of contributions that key portions of the tRNA make with the ribosome and how they influence the dynamics of the ribosome. This has led to the hypothesis that tRNAs provide a type of control during translation [10].

The process of translocation is of significant importance because of its complex structural rearrangement. Following the accommodation of tRNA into the ribosome and peptidyl bond formation, the ribosome assumes a conformation in which the subunits are rotated, the L1 stalk is closed, and the tRNAs, are in the hybrid states. This positions the ribosome in the correct 
confirmation for the binding of EF-G and, eventually, translocation to the following codon. EF-G, an important enzyme which facilitates the translocation of the ribosome by the hydrolysis of GTP, is also dynamic and helps to facilitate translocation [11]. Investigation of EF-G during translocation has revealed that it undergoes a conformational change from a more compact form to an extended conformation [12]. Studying each motion independently has provided a wealth of knowledge but how the dynamics of the ribosome work together is not well understood.

There have been a number of studies aimed at understanding how large-scale dynamics of the ribosome work together to translate protein. Understanding if and how coordination and/or correlation of large-scale dynamics is important to the mechanism of the ribosome for translation is critical. The ribosome is very large 2.5 Mega Dalton macromolecular complex that comes in contact with numerous factors and cellular environments but is still required to be able to efficiently synthesize proteins with fidelity. Early studies determined that the ribosome under goes a number of large-scale structural rearrangements, but a comprehensive understanding of how each dynamic motion fits together is still unknown.

A comparison of the rates of subunit rotation and L1 stalk motion showed that the forward rotation and closure of the L1 occur on a similar timescale. While the reverse rotation and opening of the L1 stalk do not necessarily occur on the same timescale. This suggests a number of important questions about the coordination and correlation of these two large-scale motions of the ribosome. [4]

It has been shown that when the ribosome's subunits are rotated, the L1 stalk is closed and the tRNA in the hybrid states, the ribosome is said to be in the Pre-translocation state. This can then lead to the binding of EF-G and translocation of the ribosome. Experimentation with the ribosome in the Pre-translocated state has determined that L1 stalk dynamics are not tightly coupled to tRNA motion. Rather, the closure of the L1 stalk and formation of the hybrid state by the tRNA must converge at the same time, as a stochastic multistep process, where the extent of conformational coupling depends on the nature of tRNA and bound substrate [13]. The presence 
of EF-G during smFRET experiments with ribosomes prepared in the Pre-translocation has been shown to accelerate the dynamics of the L1 stalk and tRNA of the ribosome [14]. Together the data suggests that the coupling of conformational processes on the ribosome helps to regulate translocation and translation as a whole.

We used smFRET to examine the importance of the L1 stalk-tRNA interaction toward the local and global dynamics of subunit rotation and L1 stalk motion. We mutated key residues of the L1 protein component of the L1 stalk while examining L1 stalk motion and deleted the L1 protein component of the L1 stalk while examining subunit rotation. We hypothesized that the interaction between the L1 protein component of the L1 stalk and tRNA bound to the ribosomes would influence both local and global dynamics of the ribosome. More specifically, we hypothesized that perturbations to the interface between the L1 stalk and tRNA would result in changes to the equilibrium and rates of large scale dynamics of the ribosome.

Our observation of the wild-type and mutant smFRET constructs demonstrated that the residues we chose to mutate did alter the dynamics of the L1 stalk. Additionally, we found that removal of the $\mathrm{L} 1$ protein component of the $\mathrm{L} 1$ stalk resulted in a ribosome unable to preferentially stabilize in the rotated state.

\subsubsection{Results}

\section{Preparation of fluorescently labeled ribosome constructs}

We chose to use previously established ribosomal smFRET constructs that are able to examine both subunit rotation [1] and L1 stalk motion [4]. Cysteine and alanine point mutations were made by sitedirected mutagenesis and allowed for protein labeling with fluorescent dye and the disruption of important interactions between the L1 protein component of the L1 stalk and tRNA within the ribosome. Purified proteins were conjugated to either Cy3 maleimide: L33T29C-Cy3 and L9N11C-Cy3, or Cy5 maleimide: L1K88C-Cy5, L1K88C-Cy5 R53A, L1K88C-Cy5 K54A, L1K88C-Cy5 R60A, L1K88C-Cy5 R164A, L1K88C-Cy5 R53A R60A R164A and S6D41C-Cy5. Fluorescently labeled proteins were then reassociated into their component subunit and re-purified. Both constructs were used for experimentation 
with smFRET and confirmation of activity was previously established [1,4]. Ribosomes were purified by both classical and affinity purification methods. Ribosomes used for experimentation were purified from the corresponding strains containing $\Delta \mathrm{S} 6 \mathrm{His}, \Delta \mathrm{L} 1 / \Delta \mathrm{L} 9 \mathrm{His}, \Delta \mathrm{L} 1 / \Delta \mathrm{L} 33$. In the case of the S6/L9/ $\Delta \mathrm{L} 1$ construct, ribosomal subunits were joined and re-purified by density gradient ultracentrifugation after reassociation of labeled protein. Similarly, the L1/L33 constructs were re-associated with their component labeled protein and re-purified.

\section{SmFRET experimental design}

We developed two sets of constructs to examine subunit rotation in ribosomes lacking the L1 protein and ribosome constructs that contain mutations in the $\mathrm{L} 1$ protein that report on the dynamics of the L1 stalk; both used two color smFRET. Known positively conserved residues of Arginine and Lysine were chosen for Alanine substitutions and were picked based on structural data and proposed interactions thought to be crucial in the formation the L1 protein-tRNA-tRNA interactions [15]. Residues R53, R60 and R164 are known to make contact with $\Psi 55$ of the tRNA-fMet. The mutation of the $\Psi 55 \cdot \mathrm{G} 18$ base pair resulted in an 80-fold decrease in translocation rates. Observation of the tRNA dynamics within ribosomes prepared in the Pre-translocation state but which were lacking the L1 protein component of the L1 stalk saw significant stabilization of what is called the tRNA classical state which has the A and P-site tRNA occupy the $\mathrm{A} / \mathrm{A}$ and $\mathrm{P} / \mathrm{P}$ states as opposed to the Hybrid state which is $\mathrm{A} / \mathrm{P}$ and $\mathrm{P} / \mathrm{E}$ stabilization which is typically observed [16]. These previous studies raised a number of questions that suggest that the interactions between the L1 stalk and tRNA are critical to the formation of the Pre-translocation complex, translation, and may be critical to other processes of the ribosome.

\section{SmFRET Data:}

\section{Ribosome construct that reports on L1 stalk dynamics L1 (Cy3)/L33 (Cy5)}

L1/L33 ribosomes assembled in complex with mRNA and tRNA have been shown to fluctuate between three well defined FRET states, the open state which is seen as a $\sim 0.25$ FRET state, the closed state which is seen as a $\sim 0.55$ FRET state, and half-closed state which is seen as a $\sim 0.4$ FRET state [4]. The L1 stalk's half-closed state is thought to represent an intermediate state during which the tRNAs are in the process of being removed from the ribosome. The three states, of the L1 stalk exist at an equilibrium which is freely transitioning without the addition of external factors or chemical energy. It has been shown that 
the proportion of ribosome occupying a specific distribution of states is depending on the factors involved and the stage within translation. Functionally the L1 stalk has been shown to facilitate the removal of tRNA from the E-Site of the ribosome as well as to help stabilize the ribosome throughout translation. During normal translation the ribosome primarily occupies the open and closed state of the L1 stalk at an equilibrium which is dependent on the stage of translation, the nature of the tRNAs occupying the P and A sites and the acylation status of the P site tRNA.

\section{L1 stalk motions in mutants:}

The previously tested construct L1/L33, which reports L1 stalk motions, possesses cysteine point mutations and is fluorescently labeled at L1K88C-Cy5 and L33T29C-Cy3, was chosen for examination of the L1 stalk. In addition, site-directed mutagenesis was used to change positively charge arginine and lysine residues to alanine. This included: R53A, K54A, R60A, R164A and R53A R60A R164A (triple mutant). Our intent was to remove the charge interaction between the L1 stalk and tRNA bound to the ribosome in order to disrupt their interactions, which would result in a change in the L1 stalks distribution of states, a change in the number of fluctuating molecules, and a change in rates.

\section{Investigating L1 stalk dynamics of ribosomes with alanine substitutions}

The four single point mutations and one triple mutant all had an effect on the ability of the ribosomes to form the open and closed conformation of the L1 stalk when assembled with three different preparations of tRNA. Wildtype L1/L33 ribosomes prepared with tRNA-fMet in the P-site had their L1 stalk in the open conformation $52 \%$ of the time with $40 \%$ of the traces fluctuating between the open and closed conformation. L1/L33 mutant construct R53A prepared identically was 77\% of the time within the open conformation with $41.5 \%$ of trances fluctuating between the open and closed conformation. L1/L33 mutant constructs K54A, R60A, R164A and the triple mutant were also prepared identically to wildtype but were $76 \%, 80 \%, 81 \%$ and $78 \%$ of the time in the open conformation respectively and had an increased number of fluctuating traces with $54.5 \%, 54.4 \%, 51.7 \%$ and $55.8 \%$.

Wildtype L1/L33 ribosomes prepared with tRNA-fMet in the P-site and N-acetylatedPhenylalanine-tRNA-Phenylalanine (Nac-Phe-tRNA-Phe) in the A-site had their L1 stalk $67 \%$ of the times in the open conformation with $56.7 \%$ of the traces fluctuating between the open and closed conformations. All mutant constructs, R53A, K54A, R60A, R164A and the triple mutant saw a higher proportion of 
ribosomes in the open conformation with 76\%, 75\%, 83\%, 80\% and 85\%. Mutant constructs R53A, K54A, R60A and R164A, but not the triple, saw little change in the number of fluctuating traces with $54.6 \%$, $54.6 \%, 55.6 \%, 57.6 \%$, and $57.5 \%$; whereas, the triple construct saw a decrease in the number of fluctuating traces to $35.2 \%$ when compared to the wildtype L1/L33 constructs with $56.7 \%$.

Wildtype L1/L33 ribosomes prepared with Nac-Phe-tRNA-Phe in the P site had their L1 stalk $63 \%$ of the time in the open conformation with $24.8 \%$ of the molecules fluctuating between an open and closed conformation. All of the mutant L1/L33 constructs, R53A, K54A, R60A, R164A and the triple had the L1 stalk occupy a higher proportion of the open state with $74 \%, 77 \%, 74 \%, 78 \%$ and $84 \%$ as well as an increase in the number of fluctuating traces $44.4 \%, 46.5 \%, 47.5 \%, 51.1 \%$ and $56.2 \%$. 


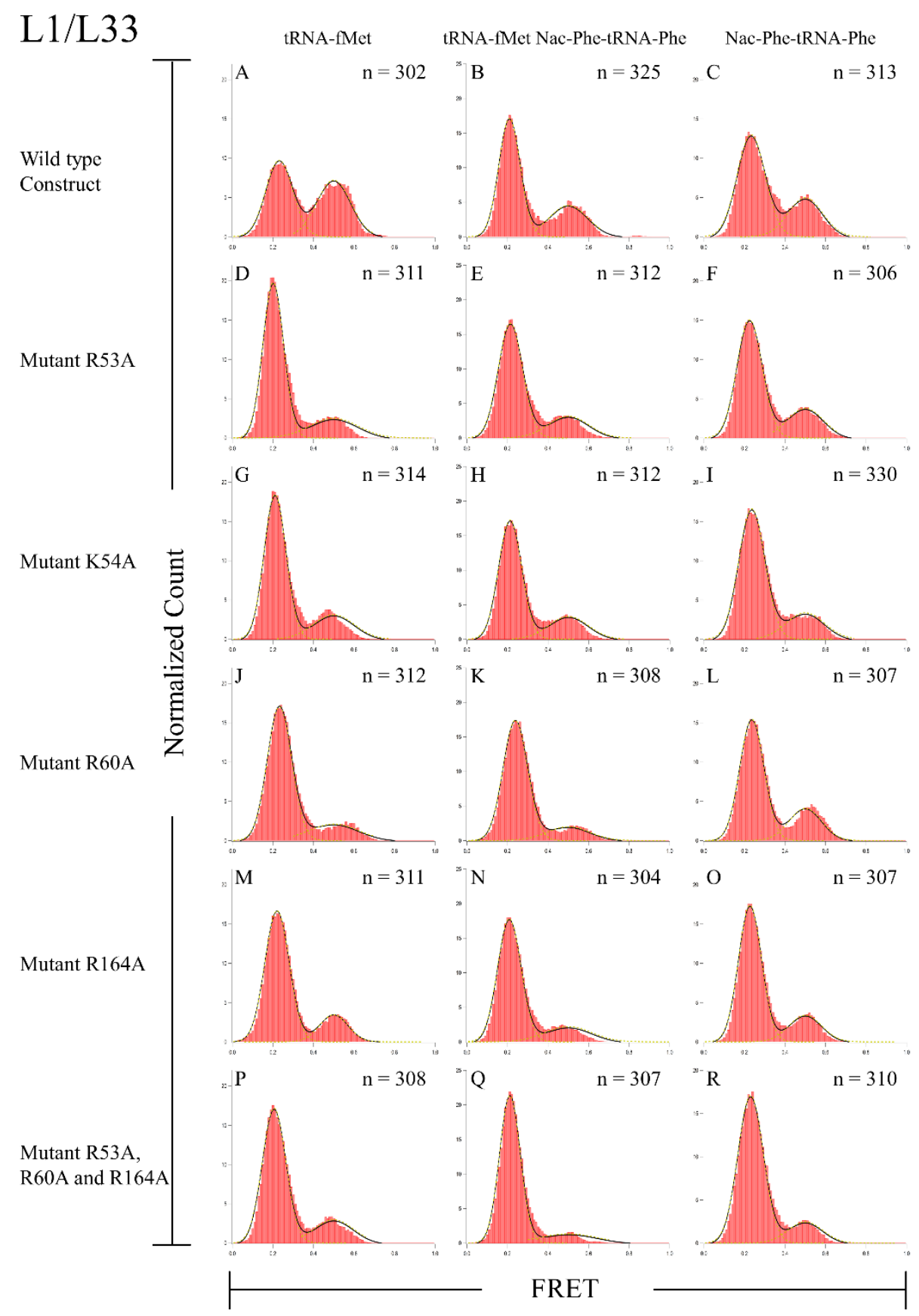

\subsubsection{Figure 35 smFRET L1/L33 data}

smFRET Histograms of the L1/L33 data for the samples: L1/L33 ribosome constructs prepared with m291 (A-C) A. tRNA-fMet B. tRNA-fMet Nac-Phe-tRNA-Phe C. Nac-Phe-tRNA-Phe. (D-F) L1/L33 R53A D. tRNA-fMet E. tRNA-fMet Nac-Phe-tRNA-Phe F. Nac-Phe-tRNA-Phe. (G-I) L1/L33 K54A G. tRNA-fMet H. tRNA-fMet Nac-Phe-tRNA-Phe I. Nac-Phe-tRNA-Phe. (J-L) L1/L33 R60A J. tRNA-fMet K. tRNAfMet Nac-Phe-tRNA-Phe L. Nac-Phe-tRNA-Phe 
(M-O) L1/L33 R164A M. tRNA-fMet N. tRNA-fMet Nac-Phe-tRNA-Phe O. Nac-Phe-tRNA-Phe. (P-R) L1/L33 triple R53A, R60A and R164A P. tRNA-fMet Q. tRNA-fMet Nac-Phe-tRNA-Phe R. Nac-PhetRNA-Phe.

\begin{tabular}{|c|c|c|c|c|}
\hline L1/L33 & $\%$ in the .2 state & $\%$ in the .5 state & $\operatorname{trans} \%$ & $\begin{array}{l}\text { Number of } \\
\text { Molecules }\end{array}$ \\
\hline tRNA-fMet & 52.07 & 47.93 & 40.00 & 302 \\
\hline tRNA-fMet/Nac-Phe-tRNA-Phe & 67.37 & 32.63 & 56.70 & 325 \\
\hline Nac-Phe-tRNA-Phe & 63.04 & 36.96 & 24.80 & 313 \\
\hline \multicolumn{5}{|l|}{ R53A } \\
\hline tRNA-fMet & 77.04 & 22.96 & 41.50 & 311 \\
\hline tRNA-fMet/Nac-Phe-tRNA-Phe & 75.95 & 24.05 & 54.60 & 312 \\
\hline Nac-Phe-tRNA-Phe & 73.63 & 26.37 & 44.40 & 306 \\
\hline \multicolumn{5}{|l|}{ K54A } \\
\hline tRNA-fMet & 75.94 & 24.06 & 54.50 & 314 \\
\hline tRNA-fMet/Nac-Phe-tRNA-Phe & 75.35 & 24.65 & 54.60 & 312 \\
\hline Nac-Phe-tRNA-Phe & 77.04 & 22.96 & 46.50 & 330 \\
\hline \multicolumn{5}{|l|}{ R60A } \\
\hline tRNA-fMet & 80.01 & 19.99 & 54.40 & 312 \\
\hline tRNA-fMet/Nac-Phe-tRNA-Phe & 83.32 & 16.68 & 55.60 & 308 \\
\hline Nac-Phe-tRNA-Phe & 73.75 & 26.25 & 47.50 & 307 \\
\hline \multicolumn{5}{|l|}{ R164A } \\
\hline tRNA-fMet & 80.56 & 19.44 & 51.70 & 311 \\
\hline tRNA-fMet/Nac-Phe-tRNA-Phe & 80.10 & 19.90 & 57.50 & 304 \\
\hline Nac-Phe-tRNA-Phe & 78.36 & 21.64 & 51.10 & 307 \\
\hline \multicolumn{5}{|l|}{ triple } \\
\hline tRNA-fMet & 77.59 & 22.41 & 55.80 & 308 \\
\hline tRNA-fMet/Nac-Phe-tRNA-Phe & 85.09 & 14.91 & 35.20 & 307 \\
\hline Nac-Phe-tRNA-Phe & 83.60 & 16.40 & 56.20 & 310 \\
\hline
\end{tabular}

\subsubsection{Table 8 Calculated values for the L1/L33 smFRET data}

Table 1: Values for the percent of the population in the $\sim 0.2$ state and the percent of the population in the $\sim 0.5$ state as well as the $\%$ of transitioning molecules and number of molecules used to build each histogram. Listed are L1/L33 wild type, L1/L33 R53A, L1/L33 K54A, L1/L33 R60A, L1/L33 R164A and L1/L33 triple. Histograms for tRNA preparation of, tRNA-fMet, tRNA-fMet Nac-Phe-tRNA-Phe and NacPhe-tRNA-Phe were analyzed for each construct. 


\section{L1/L33 mutant ribosome construct smFRET rate analysis}

The traces that contained fluctuations for each construct were subjected to Hammy and TDP to determine the rate of L1 closure and opening. (Table 2) Tabulated are the rates of L1 stalk closure $\mathrm{k}_{1}$ and rate of $\mathrm{L} 1$ stalk opening $\mathrm{k}_{-1}$. The rate was determined by a Gaussian fit of the Hammy data by TDP and the Width column represents the variability of the data.

\begin{tabular}{|c|c|c|c|c|c|c|}
\hline & $\mathrm{k}_{1}$ & Closure & & $\mathrm{k}_{-1}$ & Opening & \\
\hline $\mathrm{L} 1 / \mathrm{L} 33 \mathrm{Wt}$ & Rate $\left(\mathrm{s}^{-1}\right)$ : & Width $\left(\mathrm{s}^{-1}\right)$ : & \# of trans: & Rate $\left(\mathrm{s}^{-1}\right)$ : & Width $\left(\mathrm{s}^{-1}\right)$ : & \# of trans: \\
\hline tRNA-fMet & 0.108 & 0.027 & 103 & 0.539 & 0.412 & 94 \\
\hline $\begin{array}{l}\text { tRNA-fMet Nac- } \\
\text { Phe-tRNA-Phe }\end{array}$ & 0.239 & 0.111 & 221 & 0.878 & 0.487 & 207 \\
\hline Nac-Phe-tRNA-Phe & 0.464 & 0.306 & 85 & 0.875 & 0.219 & 80 \\
\hline L1/L33 R53A & Rate $\left(\mathrm{s}^{-1}\right)$ : & Width $\left(\mathrm{s}^{-1}\right)$ : & \# of trans: & Rate $\left(\mathrm{s}^{-1}\right)$ : & Width $\left(\mathrm{s}^{-1}\right)$ : & \# of trans: \\
\hline tRNA-fMet & 0.136 & 0.136 & 219 & 0.574 & 0.598 & 212 \\
\hline $\begin{array}{l}\text { tRNA-fMet Nac- } \\
\text { Phe-tRNA-Phe }\end{array}$ & 0.181 & 0.204 & 429 & 0.670 & 0.479 & 425 \\
\hline Nac-Phe-tRNA-Phe & 0.371 & 0.110 & 357 & 0.337 & 0.084 & 367 \\
\hline L1/L33 K54A & Rate $\left(\mathrm{s}^{-1}\right)$ : & Width $\left(\mathrm{s}^{-1}\right)$ : & \# of trans: & Rate $\left(\mathrm{s}^{-1}\right)$ : & Width $\left(\mathrm{s}^{-1}\right)$ : & \# of trans: \\
\hline tRNA-fMet & 0.133 & 0.156 & 253 & 0.482 & 0.507 & 244 \\
\hline $\begin{array}{l}\text { tRNA-fMet Nac- } \\
\text { Phe-tRNA-Phe }\end{array}$ & 0.300 & 0.081 & 250 & 0.819 & 0.620 & 241 \\
\hline Nac-Phe-tRNA-Phe & 0.569 & 0.142 & 196 & 0.479 & 0.120 & 185 \\
\hline L1/L33 R60A & Rate $\left(\mathrm{s}^{-1}\right)$ : & Width $\left(\mathrm{s}^{-1}\right)$ : & \# of trans: & Rate $\left(\mathrm{s}^{-1}\right)$ : & Width $\left(\mathrm{s}^{-1}\right)$ : & \# of trans: \\
\hline tRNA-fMet & 0.174 & 0.165 & 208 & 0.860 & 0.333 & 204 \\
\hline $\begin{array}{l}\text { tRNA-fMet Nac- } \\
\text { Phe-tRNA-Phe }\end{array}$ & 0.357 & 0.123 & 252 & 0.465 & 0.116 & 243 \\
\hline Nac-Phe-tRNA-Phe & 0.336 & 0.263 & 309 & 0.741 & 0.453 & 300 \\
\hline L1/L33 R164A & Rate $\left(\mathrm{s}^{-1}\right)$ : & Width $\left(\mathrm{s}^{-1}\right)$ : & \# of trans: & Rate $\left(\mathrm{s}^{-1}\right)$ : & Width $\left(\mathrm{s}^{-1}\right)$ : & \# of trans: \\
\hline tRNA-fMet & 0.172 & 0.211 & 241 & 0.575 & 0.579 & 246 \\
\hline $\begin{array}{l}\text { tRNA-fMet Nac- } \\
\text { Phe-tRNA-Phe }\end{array}$ & 0.163 & 0.198 & 249 & 0.565 & 0.625 & 235 \\
\hline Nac-Phe-tRNA-Phe & 0.434 & 0.472 & 219 & 0.743 & 0.339 & 205 \\
\hline L1/L33 triple & Rate $\left(\mathrm{s}^{-1}\right)$ : & Width $\left(\mathrm{s}^{-1}\right)$ : & \# of trans: & Rate $\left(\mathrm{s}^{-1}\right)$ : & Width $\left(\mathrm{s}^{-1}\right)$ : & \# of trans: \\
\hline tRNA-fMet & 0.145 & 0.138 & 295 & 1.095 & 0.521 & 285 \\
\hline $\begin{array}{l}\text { tRNA-fMet Nac- } \\
\text { Phe-tRNA-Phe }\end{array}$ & 0.213 & 0.257 & 198 & 0.866 & 0.217 & 193 \\
\hline Nac-Phe-tRNA-Phe & 0.233 & 0.058 & 194 & 1.516 & 0.379 & 203 \\
\hline
\end{tabular}

\subsubsection{Table 9 Calculated rates for the L1/L33 constructs}

Table 2 Calculated rates for the five mutant constructs and the wild type L1/L33 construct: R53A, K54A, R60A, R164A and triple (R53A R60A and R164A) 
The forward and reverse rates for $\mathrm{L} 1$ stalk motion are significantly different between the $\mathrm{Wt}$ L1/L33 construct and the mutant L1/L33 constructs when assembled with either tRNA-fMet, or tRNAfMet, Nac-phe-tRNA-Phe or Nac-phe-tRNA-Phe (5.3.0 Table 9). An increase in the forward rate, which is the transition from the open to closed conformation, was seems for all of the mutant constructs except R60A and R164A when assembled with tRNA-fMet. The largest change from Wt $0.108 \mathrm{~s}^{-1} \pm 0.027$ was for R60A $0.174 \mathrm{~s}^{-1} \pm 0.165$ and R164A $0.172 \mathrm{~s}^{-1} \pm 0.211$. The other constructs forward rate also changed to lesser degree: R53A $0.136 \mathrm{~s}^{-1}+-0.136$, K54A $0.133 \mathrm{~s}^{-1} \pm 0.156$, and triple $0.145 \mathrm{~s}^{-1} \pm 0.138$.

An increase in the reverse rate, which is the transition from the closed to the open conformation, was seen for all of the mutant constructs except for K54A when assembled with tRNA-fMet. The Wt L1/L33 reverse rate was reported as $0.539 \mathrm{~s}^{-1} \pm 0.412$ while the K54A mutant rate was $0.482 \mathrm{~s}^{-1} \pm 0.507$ and the triple mutant's rate reverse rate was $1.095 \mathrm{~s}^{-1} \pm 0.521$. The reverse rate increased for R53A $0.574 \mathrm{~s}^{-1} \pm$ $0.598, \mathrm{R} 60 \mathrm{~A} 0.860 \mathrm{~s}^{-1} \pm 0.333$ and R164A $0.575 \mathrm{~s}^{-1} \pm 0.579$.

The forward rate increased or decreased depending on which L1/L33 mutant construct was assembled with tRNA-fMet and Nac-Phe-tRNA-Phe. K54A $0.300 \mathrm{~s}^{-1} \pm 0.081$ and R60A $0.357 \mathrm{~s}^{-1} \pm 0.123$ had increased rates from the Wt $0.239 \mathrm{~s}^{-1} \pm 0.11$. The mutant constructs R53A $0.181 \mathrm{~s}^{-1} \pm 0.204$, R164A $0.163 \mathrm{~s}^{-1} \pm 0.198$ and the triple $0.213 \mathrm{~s}^{-1} \pm 0.257$ had decreased rates when compared to the Wt $0.239 \mathrm{~s}^{-1} \pm$ 0.11 .

A decrease in the reverse rate was seen for all of the L1/L33 mutant constructs when assembled with tRNA-fMet and Nac-Phe-tRNA-Phe. The K54A $0.819 \mathrm{~s}^{-1} \pm 0.620$ and triple $0.819 \mathrm{~s}^{-1} \pm 0.620$ mutant constructs had a slight decrease in their rate when compared to the Wt $0.878 \mathrm{~s}^{-1} \pm 0.487$. The R53A0.670 $\mathrm{s}^{-1}$ +- 0.479, R60A $0.465 \mathrm{~s}^{-1} \pm 0.116$ and R164A $0.565 \mathrm{~s}^{-1} \pm 0.625$ mutant constructs all saw a larger decrease in their rate.

A decrease in the forward rate was seen for the L1/L33 mutant constructs assembled with NacPhe-tRNA-Phe, excluding the K54A mutant construct which had an increased rate. The R53A $0.371 \mathrm{~s}^{-1} \pm$ 0.110, R60A $0.336 \mathrm{~s}^{-1} \pm 0.263$ and R164A $0.434 \mathrm{~s}^{-1} \pm 0.472$ rates were slightly decreased but remained similar to the Wt $0.464 \mathrm{~s}^{-1} \pm 0.306$. The K54A $0.569 \mathrm{~s}^{-1} \pm 0.142$ mutant construct had a forward rate when 
compared to the Wt construct. The most drastic change was the triple $0.233 \mathrm{~s}^{-1} \pm 0.058$ which decreased to almost half of the rate of the Wt construct.

A decrease in the reverse rates we seen for the L1/L33 mutant constructs except the triple which had an increased reverse rate when assembled with Nac-Phe-tRNA-Phe. The R53A $0.337 \mathrm{~s}^{-1} \pm 0.084$ and K54A $0.479 \mathrm{~s}^{-1} \pm 0.120$ mutant constructs had a large decrease in their reverse rate form the Wt $0.875 \mathrm{~s}^{-1} \pm$ 0.219. A smaller decrease was seen for R60A $0.741 \mathrm{~s}^{-1} \pm 0.453$ and R164A $0.743 \mathrm{~s}^{-1} \pm 0.339$ mutant constructs. The triple $1.516 \mathrm{~s}^{-1} \pm 0.379$ mutant construct almost doubled the rate of the Wt sample.

\section{Ribosome construct that reports on inter-subunit rotation $\mathrm{S6}(\mathrm{Cy5}) / \mathrm{L9}(\mathrm{Cy3})$}

S6/L9 ribosomes assembled in complex with mRNA and tRNA have been shown to fluctuate between two well defined states, the non-rotated $(\sim 0.58)$ and the rotated $(\sim 0.38)$ states [1]. The addition of a downstream element during translation has been shown to drive the ribosome into what has been termed the hyper-rotated state which has a FRET state of ( 0.22). [18] The ribosome has also been shown to be able to occupy what has been termed the semi rotated conformation which has a ( 0.5) FRET state and has been shown to be induced by initiation factor 2 during initiation of the ribosome [19].

\section{Observations of subunit rotation with ribosomes lacking the L1 protein component of the}

\section{L1 stalk}

We generated the ribosomal construct S6/L9 which lacked the L1 protein component of the L1 stalk through genomic deletion. SmFRET experiments demonstrate ribosomes lacking the L1 stalk were unable to efficiently occupy the rotated state under normal circumstances.

Wildtype S6/L9 ribosomes prepared with tRNA-fMet in the P-site were primarily in the rotated conformation with $77 \%$ of the molecules occupying the low FRET state and $70 \%$ of the traces containing transitions. With a transition being defined as a single dynamic event between the two known states, the S6/L9 ribosomes lacking the $\mathrm{L} 1$ protein component of the L1 stalk occupied the rotated state $41 \%$ of the time and a similar amount of fluctuating traces with $71 \%$.

Wildtype S6/L9 ribosomes prepared with tRNA-fMet in the P-site and Nac-Phe-tRNA-Phe is the A-site occupied the rotated state $69 \%$ of the time with $59 \%$ of molecules having fluctuations. S6/L9 ribosomes lacking the $\mathrm{L} 1$ protein component of the $\mathrm{L} 1$ stalk occupied the rotated state $37 \%$ of the time and with $54 \%$ of molecules having transitions. 
Wildtype S6/L9 ribosomes prepared with Nac-Phe-tRNA-Phe in the P-site were in the rotated state $25 \%$ of the time with $4 \%$ of their molecules containing transitions. S6/L9 ribosomes lacking the L1 component of the L1 stalk were in the rotated state $44 \%$ of the time with $50 \%$ of their molecules containing transitions.

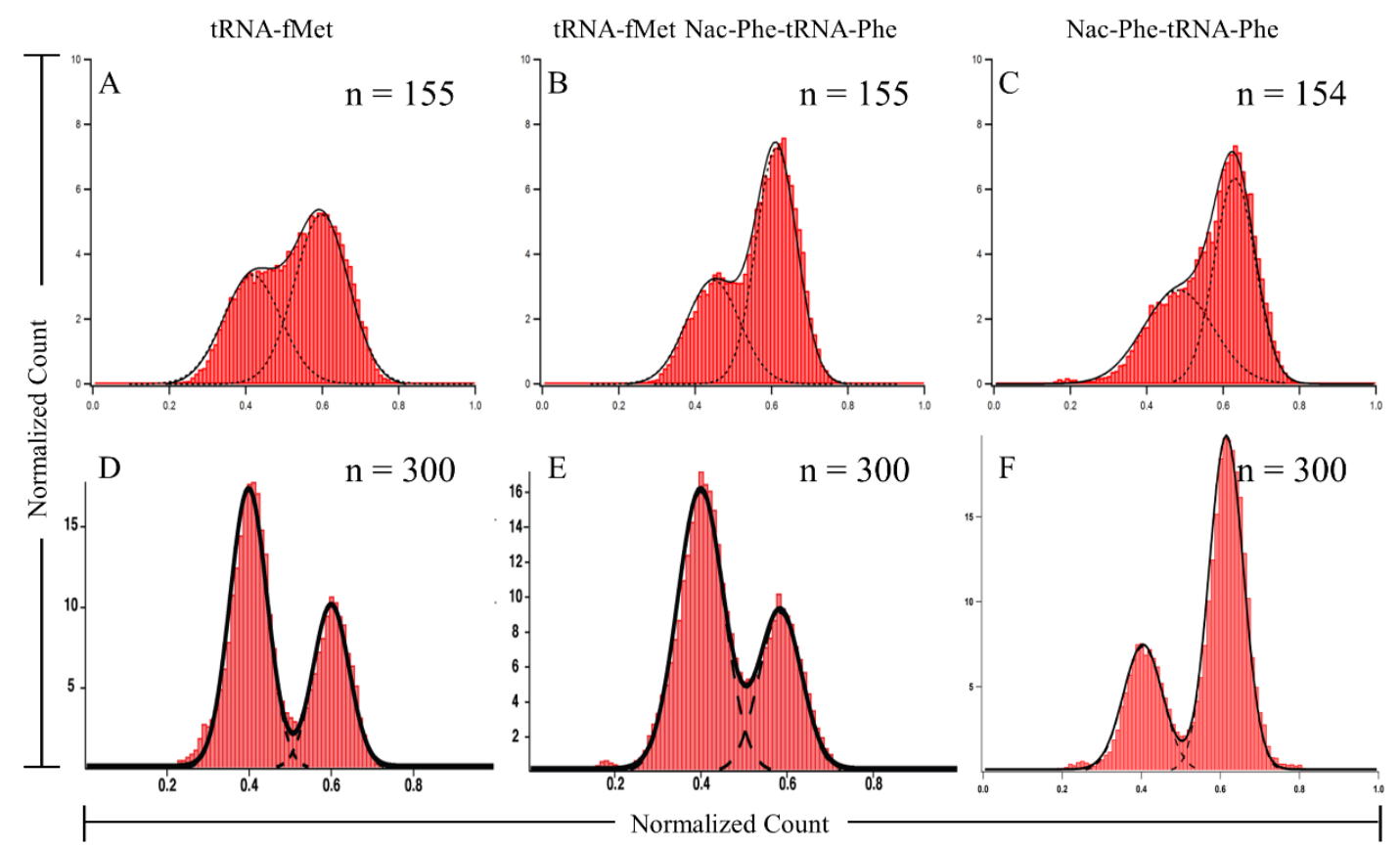

\subsubsection{Figure 36 S6/L9/AL1 smFRET data}

Figure 2 S6/L9/AL1 smFRET data: A-C S6/L9/AL1 preparations of ribosomes with tRNA-fMet, tRNA-

fMet, Nac-Phe-tRNA-Phe and Nac-Phe-tRNA-Phe. L1 D-F S6/L9 tRNA-fMet, tRNA-fMet, Nac-PhetRNA-Phe and Nac-Phe-tRNA-Phe.

\begin{tabular}{|l|l|l|l|}
\hline & \% Rotated & \% Not rotated & \% of traces with transitions \\
\hline S6/L9/AL1 & & & \\
\hline tRNA-fMet & 41 & 59 & 70 \\
\hline tRNA-fMet Nac-Phe-tRNA-Phe & 37 & 63 & 54 \\
\hline Nac-Phe-tRNA-Phe & 44 & 56 & 50 \\
\hline S6/L9 & & & \\
\hline tRNA-fMet & 77 & 23 & 71 \\
\hline tRNA-fMet Nac-Phe-tRNA-Phe & 69 & 31 & 59 \\
\hline Nac-Phe-tRNA-Phe & 25 & 75 & 4 \\
\hline
\end{tabular}




\subsubsection{Table 10 Calculates values for the S6/L9/ $\Delta \mathrm{L} 1 \mathrm{smFRET}$ data}

Table 3: Values for the $\%$ of the population in the $\sim 0.38$ state and the $\%$ of the population in the $\sim 0.58$ state and the $\%$ of molecules with transitions. Listed are $\mathrm{S} 6 / \mathrm{L} 9 / \Delta \mathrm{L} 1$ and $\mathrm{S} 6 / \mathrm{L} 9$ ribosomes prepared with, tRNAfMet, tRNA-fMet Nac-Phe-tRNA-Phe and Nac-Phe-tRNA-Phe.

\subsubsection{Material and Methods:}

\section{Classical ribosome purification}

$70 \mathrm{~S}$ ribosomes carrying the $\Delta \mathrm{L} 1 / \Delta \mathrm{L} 33$ genomic deletions were purified as mentioned in Chapter 4 section title: Classical purification of tight-coupled ribosomes and subunits.

\section{Affinity purification of $50 S$ and $30 S$ subunits}

Ribosomal subunits $\Delta \mathrm{S} 6$ His and $\Delta \mathrm{L} 1 / \Delta \mathrm{L} 9$ His were purified based the protocol found in Chapter 4 chapter's section title: Affinity purification of $50 \mathrm{~S}$ and $30 \mathrm{~S}$ subunits.

\section{Protein purification}

Ribosomal proteins S6, L9, L1 and L33 were cloned from E. coli strain BW25113 into Pet11a using Cloning sites BamHI and NdeI. The cloning was verified by sequencing. The generations of mutant constructs containing single cysteine residues to be used for labeling were made by site directed mutagenesis and are S6D41C, L9N11C, L1K88C, L33T29C, L1K88C R53A, L1K88C K54A, L1K88C R60A, L1K88C R164A and L1K88C R53A R60A R164A. All constructs were verified by sequencing.

\section{Purification of S6D41C}

S6D41C was purified by the protocol found in Chapter 4 section title: Purification of S6D41C.

\section{Purification of L9N11C}

L9N11C was purified by the protocol found in Chapter 4 section title: Purification of L9N11C.

\section{Purification of ribosomal proteins L1K88C, L33T29C, L1K88C R53A, L1K88C K54A,}

\section{L1K88C R60A, L1K88C R164A and L1K88C R53A R60A R164A}

Following cloning, rplA (L1 gene) mutants and rpmG (L33 gene) mutants were expressed in E. coli BL21 (DE3) by growth in 1L LB to an OD600 of $.4-.6$ at $27^{\circ} \mathrm{C}$. Cultures were induced for $4 \mathrm{~h}$ with $1 \mathrm{mM}$ IPTG. Cells were pelleted in an SLC-6000 Sorvall rotor at $3000 \times \mathrm{g}$ for $20 \mathrm{~min}$ and stored at $-20^{\circ} \mathrm{C}$. Cells were resuspended in Buffer $\mathrm{C}$ and lysed by French Press. The lysate was then clarified at 12,000 
RPM for 15 min in a Sorvall SS-34 rotor at $4{ }^{\circ} \mathrm{C}$. The pellet containing inclusion bodies was then dissolved in $10 \mathrm{~mL}$ of buffer B. After solubilizing the inclusion bodies, the sample was dialyzed $3 \times$ against buffer A at $4{ }^{\circ} \mathrm{C}$. The sample was then clarified by centrifugation at 12,000 RPM for $15 \mathrm{~min}$ in an SS-34 rotor at 4 ${ }^{\circ} \mathrm{C}$ and filtered through a $0.8 \mu \mathrm{m}$ cellulose acetate syringe filter. The protein was purified by a Resource $\mathrm{S}$ cation exchange column with a 20x column volume linear gradient 0-30\% of buffer B in buffer A. Protein obtained by this method was then dialyzed into buffer R, concentrated and frozen at $-80{ }^{\circ} \mathrm{C}$.

\section{Generation of the $\Delta \mathrm{S} 6 \mathrm{His}$ and $\Delta \mathrm{L} 1 / \Delta \mathrm{L} 9 \mathrm{His}$ constructs}

The protocols for generating the $\Delta \mathrm{L} 1 / \Delta \mathrm{L} 9$ His tagged ribosomes and $\Delta \mathrm{S} 6$ His tagged construct can be found in Chapter 4 section title: Construction of the knockout strains, or can be obtained through the following citation, P1 phage transduction and related protocols [20].

\section{Ribosomal construct $\Delta \mathrm{L} 1 / \Delta \mathrm{L} 33$}

Ribosomes containing $\Delta \mathrm{L} 1 / \Delta \mathrm{L} 33$ genomic deletions were a gift from the Noller lab [4].

\section{Labeling of S6D41C and L9N11C}

Protocols for labeling of S6D41C and L9N11C can be found in Chapter 4 section title: Fluorescent labeling of ribosomal proteins.

\section{Fluorescent labeling L1K88C and L33T29C}

Labeling of L1K88C, L33T29C, L1K88C R53A, L1K88C K54A, L1K88C R60A, L1K88C

R164A and L1K88C R53A R60A R164A were accomplished by using the protocol to L9N11C which can be found in Chapter 4 section title: Fluorescent labeling of ribosomal proteins.

\section{Construct assembly}

\section{Generation of $\mathrm{L} 1 / \mathrm{L} 33$ constructs}

All of the L1/L33 constructs were made in an identical method to those generated previously [4]. 1.2 nmols of $\Delta \mathrm{L} 1 / \Delta \mathrm{L} 3370 \mathrm{~S}$ ribosomes were incubated with $1.8 \mathrm{nmols}$ of one of the following L1 proteins: L1K88C-Cy5, L1K88C-Cy5 R53A, L1K88C-Cy5 K54A, L1K88C-Cy5 R60A, L1K88C-Cy5 R164A and L1K88C-Cy5 R53A R60A R164A and L33T29C-Cy3 for 15 min at $4{ }^{\circ} \mathrm{C}$ in 300ul of Buffer H. Ribosomes were then washed and exchanged into Buffer G by 100 K MWCO Millipore Centricon® with 3-4, 1:10 dilutions.

\section{Generation of $\mathrm{S6} / \mathrm{L} 9 / \Delta \mathrm{L} 1$ construct}


1.2 nmoles of $\Delta$ S6 30S was re-associated with 1.8 nmoles S6D41C-Cy5 in a total volume of 300ul of Buffer $\mathrm{E}$ and incubated at $37^{\circ} \mathrm{C}$ for $15 \mathrm{~min}$. 1.2 nmoles of $\Delta \mathrm{L} 1 / \Delta \mathrm{L} 950 \mathrm{~S}$ was re-associated with 1.8 nmoles L9N11C-Cy3 in a total volume of 300ul of Buffer F and incubated at $37{ }^{\circ} \mathrm{C}$ for $15 \mathrm{~min}$. Both subunits were exchanged into Buffer G using a 100 K MWCO Millipore Centricon® with 3-4, 1:10 dilutions. The 30S S6D41C-Cy5 was then joined to the $\Delta \mathrm{L} 1 / 50 \mathrm{~S}$ L9N11C-Cy3 in a final volume of $300 u$ l with a 15 minute incubation. 1.2nmols was then split between two Sw41 tubes of 10-30\% sucrose gradients made with Buffer $\mathrm{G}$ and centrifuged at $71,000 \times \mathrm{g}$ for $14 \mathrm{~h}$ at $4{ }^{\circ} \mathrm{C}$. The gradients were pumped by the addition of $40 \%$ sucrose to the bottom of the gradient using a Brandel density gradient fractionator while monitoring the absorbance at $260 \mathrm{~nm}$ to identify the peaks corresponding to $30 \mathrm{~S}, 50 \mathrm{~S}$ and $70 \mathrm{~S}$ ribosome.

\section{Preparation of charged tRNA and mRNA}

The mRNA, m291 a derivative of T4 bacteriophage gene 32 was prepared via in-vritro transcription using a purified PCR template and denaturing polyacrylamide gel electrophoresis. tRNA, tRNA-fMet and Nac-Phe-tRNA-Phe were prepared by previously established methods [1, 4] using commercially available tRNA purchased from MP Biomedicals and tRNA probes.

\section{Assembly of ribosome complexes for smFRET}

All experiments were performed in buffer I at room temperature $\left(\sim 21^{\circ} \mathrm{c}\right)$. Ribosomal constructs S6/L9/AL1 and L1/L33 were assembled under equilibrium conditions with mRNA, m291 or deacylated tRNA, acylated tRNA or N-actylated tRNA in the A and P site of the ribosome as previously described [1, $4]$.

\section{Acquisition of smFRET data}

SmFRET experiments were performed on our home build two color smFRET instrument. Images were taken using an Andor iXonEM + 897 Electron Multiplying Charge Coupled Device (EMCCD) camera with the Cy3 and Cy5 emission sorted by a 630dcxr dichroic mirror (Chroma) with the images set side by side on the camera. Movies were taken using the custom acquisition software single, which was first developed in the Ha lab [23]. Data showing FRET between the donor and acceptor and photobleaching events for both dyes were selected for further analysis.

\section{Analysis of smFRET data}


Movies were further analyzed using custom IDL software to correlate single molecules overtime. Traces were visualized and individually analyzed using custom MatLab software, used to differentiate between traces with and without fluctuations. Once $\sim 300$ traces were chosen from the data, the data was smoothed, binned and built into histograms using IGOR. The generation of figures and fitting of the data was accomplished using IGOR's built in multipeak fitting 2 package. The peak location of L1/L33 constructs was constrained for to a FRET value of 0.5 . The number of fluctuating traces was taken directly from the Matlab analysis while the distribution of states was calculated from the area under the fit Gaussian peaks for each histogram. Rates were calculated using Hammy and TDP as previously explained above.

\section{Buffer List}

Buffer A: Low Salt Buffer: $20 \mathrm{mM}$ Tris $\mathrm{HCl}(\mathrm{pH} 7.5)$ at $4^{\circ} \mathrm{c} 20 \mathrm{mM} \mathrm{KCl} 6 \mathrm{M}$ UREA $6 \mathrm{mM} \beta \mathrm{ME}$

Buffer B: High Salt Buffer: 20mM Tris $\mathrm{HCl}(\mathrm{pH} 7.5)$ at $4^{\circ} \mathrm{c} 1 \mathrm{M} \mathrm{KCl} 6 \mathrm{M}$ UREA $6 \mathrm{mM} \beta \mathrm{ME}$

Buffer C: $80 \mathrm{mM}$ HEPES-KOH (pH 7.6), $20 \mathrm{mM} \mathrm{KCl}$, and $6 \mathrm{mM} \beta \mathrm{ME}$

Buffer D: 50 mM HEPES-KOH (pH 7.5), 4 mM MgCl2 400 mM NH4Cl, 6 mM $\beta$ ME \& Nikkol 0.01\% w/v Buffer E: 80 mM HEPES-KOH (pH 7.6), 20 mM MgCl2, 330 mM KCl, 6 mM $\beta$ ME \& Nikkol 0.01\% w/v

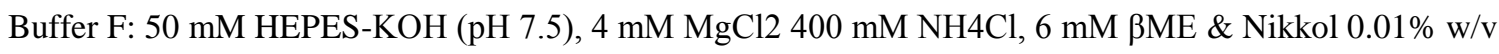
Buffer G 20 mM HEPES-KOH (pH 7.5), 20 mM MgCl2, 100 mM NH4Cl, 6 mM ßME \& Nikkol 0.01\% w/v, 2 mM Spermidine, $0.1 \mathrm{mM}$ Spermine

Buffer H 20 mM Hepes· KOH (pH 7.5), 4 mM MgCl2, 330 mM NH4Cl, 6 mM $\beta$ ME, \& 0.1\% Nikkol Buffer I 20 mM HEPES-KOH (pH 7.5), 6 mM MgCl2, 150 mM NH4Cl, 6 mM $\beta$ ME, 2 mM spermidine, and $0.1 \mathrm{mM}$ spermine

\subsubsection{Discussion}

The importance of the L1 stalk-tRNA interaction toward local and global dynamics of the ribosomes during translation is not fully understood. Using two color smFRET, combined with site-directed mutagenesis and ribosomal protein deletion, we have shown that disruptions in the L1 stalk-tRNA interaction resulted in a drastic change in the distribution of states and number of dynamic molecules for L1 stalk motion and subunit rotation. The residues we chose to mutate had various effects on the distribution of states of the L1 stalk, the number of dynamic molecules and rate. This was also the case for the deletion of the L1 protein component of the L1 stalk for which we observed the disruption of subunit rotation by a 
change in the equilibrium of states and number of dynamic molecules. These changes in the ribosome's structure suggest that our targeted approach altered the ribosome's energy landscape, and additional analysis could provide valuable insight into global dynamics of the ribosome. Furthermore, these experiments highlight the importance of the L1 stalk-tRNA interaction, showing that it is critical to the mechanism of translation by the ribosome.

The L1 stalk has been shown to be involved in the removal of E-site tRNA and to help to facilitate tRNA hybrid state formation [16]. A better understanding of the interaction between the L1 stalk, both RNA and protein components, and tRNA within the ribosome will help to better explain the mechanism of the ribosome. This includes the thermodynamic landscape of the ribosome and how factors influence and tune the ribosomes during translation. The L1 stalk-tRNA interaction may also be important in additional processes like ribosomal frameshifting, ribosomal pausing and termination in a manner not yet fully understood.

\section{Disruption of the L1 stalk-tRNA interactions remaps the energy landscape of the ribosome}

L1/L33 constructs containing mutations of lysine and arginine residues to alanine at known positions shown to interact with tRNA bound to the ribosome were probed with two color smFRET. Histograms, built from the single molecule traces for all of the generated mutations showed an altered distribution of states and changes in the number of fluctuating molecules as compared to the wild type. Furthermore, constructs that were generated without the L1 protein component of the L1 stalk showed drastic changes in the ribosomes ability to occupy the rotated conformation with all of the tRNA combinations used during experimentation. These results indicate that the interface between the L1 stalk and tRNA inside the ribosome is an important component in L1 stalk dynamics, its interaction plays an important role in the ribosome's ability to assume the correct state and the mechanism of the ribosome. Furthermore, observations of the construct examining subunit rotation but that lacked the L1 protein component of the L1 stalk demonstrated that the L1 protein component of the L1 stalk-tRNA interaction is integral to correct subunit rotation state formation and global conformational dynamics of the ribosome.

Investigating L1 stalk dynamics of ribosomes with alanine substitution within the L1 protein 
Preparation of L1/L33 ribosome constructs containing mutations at R53A, K54A, R60A, R164A and the triple mutant all saw an increase in time molecules spent in the open conformation, $77 \%, 76 \%$, $80 \%, 81 \%$ and $78 \%$ respectively as compared to the wildtype $52 \%$. This would indicate a destabilization of interactions between the L1 protein and tRNA-fMet in the P-site of the ribosome. L1/L33 construct R53A, however, did not see a change in the number of fluctuating traces, with $41.5 \%$ as compared to the wildtype 40\%, while K54A, R60A, R164A, and the triple mutant all saw an increase in the number of traces containing fluctuations with $54.5 \%, 54.4 \%, 51.7 \%$ and $55.8 \%$.

L1/L33 mutant ribosome constructs prepared with tRNA-fMet in the P-site and Nac-Phe-tRNAPhe in the A-site which mimics Pre-translocated ribosomes saw an increase in the number of the ribosomes occupying the open conformation of the L1 stalk for R53A, K54A, R60A, R164A and triple with 76\%, $75 \%, 83 \%, 80 \%$ and $85 \%$ respectively. Mutant constructs, R53A, K54A, R60A and R164A except for the triple saw little to no change in the number of fluctuating traces with $54.6 \%, 54.6 \%, 55.6 \%, 57.6 \%$, and $57.5 \%$ whereas the triple construct saw a decrease in the number of fluctuating traces to $35.2 \%$ when compared to the wildtype L1/L33 constructs with 56.7\%. Our smFRET experiment reported that the L1 occupied the open state 67\%; however, this differs from the reported literature value of $75 \%$ [4]. This is consistent with literature that suggests tRNA hybrid state formation, L1 stalk closure and the movement of the ribosomal subunits are loosely coupled but must independently come together to allow for EF-G binding [14]. In this experiment disruption of the interaction between the L1 stalk and tRNA within the ribosome resulted in varying degrees of disruption for the distribution of states and a change in the number of transitions for the triple mutant.

L1/L33 mutant ribosome constructs prepared with Nac-Phe-tRNA-Phe in the P-site which mimics post-translocated ribosomes had a higher proportion of the L1 stalk occupy the open conformation for R53A, K54A, R60A, R164A and the triple with 74\%, 77\%, 74\%, 78\% and 84\%, respectively, as compared to the wildtype $63 \%$. There was a larger change in the number of fluctuating molecules from the wildtype of $24.8 \%$ to the mutant R53A, K54A, R60A, R164A and the triple of 44.4\%, 46.5\%, 47.5\%, 51.1\% and $56.2 \%$. Our data suggest that disruption of the L1 stalk-tRNA interaction causes the ribosome to be unable to correctly form the closed state of the L1 stalk.

\section{The disruption of charge-charge interactions with the use of alanine substitution}


For the preparation of the L1/L33 mutant constructs, the choice to mutate specific lysine and arginine residues aimed to remove the charge interaction between the positive amino acids and negative phosphodiester backbone of the interacting tRNA. Alternative strategy to probe and disrupt the L1 stalktRNA interaction that could be employed, include, the addition of more positive residues or negatively charged residues to try to more tightly bind or repel the tRNA. The use of large hydrophobic residues meant to induce steric clash could be used to disrupt the formation of interactions or repel the tRNA and might provide interesting results.

\section{The L1 stalk-tRNA is an essential to the global dynamics of the ribosome}

Whether examining L1 stalk motions with L1protein mutations at the L1 protein-tRNA interface or examining subunit rotation with ribosomes lacking the $\mathrm{L} 1$ protein, both show disruptions in their distribution of states and often have a change in the number of fluctuating traces. This would suggest that the $\mathrm{L} 1$ protein component of the L1 stalk is critical for global dynamics of the ribosome through stabilization of tRNA within the ribosomes. These results are consistent with current opinions within the field including those that have studied the coordination of the L1 stalk with tRNA motion within the ribosome [16]. Large-scale dynamic motions of the ribosome are not necessarily coordinated but rather contribute to a network of interactions that influence their ability to populate specific states.

\section{Structural analysis}

Analysis of the interactions between the L1 stalk-tRNA was previously performed using X-ray crystallographic and CryoEM structural data from Thermus thermophiles by the Noller lab [3]. Four main structures were examined including the ribosome in the classical state with a vacant $\mathrm{E}$ site (PDB ID: 4GD2), Hybrid state with the (P/E) arrangement (PDB ID: 4V9H), Chimeric Hybrid with the (pe/E) arrangement (PDB ID: 4V9K), and Classical (E/E) arrangement (PDB ID: 4V67). We considered the Hybrid (P/E) arrangement bound with EF-G just prior to translocation to a good representation of the closed state of the L1 stalk.

We examined the Hybrid (P/E) arrangement with tRNA-Met, Nac-Phe-tRNA-Phe, and with EF-G bound to the Escherichia coli 70S (PDB ID: 4V7D) to examine interacting residues between the L1 protein and tRNA bound to the ribosome. For our experiments, we used tRNA-fMet instead of tRNA-Met although there are some minor differences between the two structures in Escherichia coli [24]. The structure shows 
residues 53R and 54K interacting with the T loop of tRNA-Met and appears to interact with the sugar edge of residue $61 \mathrm{C}, 62 \mathrm{C}, 63 \mathrm{C}, 64 \mathrm{G}, 54 \mathrm{~T}$, and $55 \psi$ of the tRNA. Although they are far apart in sequence, residues $60 \mathrm{R}$ and $164 \mathrm{R}$ are situated right next to one another in space, and look as if they interact with the phosphodiester backbone of residues 54T and $55 \psi$ of tRNA-Met. The L1 stalk has been shown to make further interactions with the tRNA bound to the P-site during its removal from the E-site through a variety of intermediate states [15]. Disruption of primary interacting partners, as shown above, would help to destabilize the closed state of the L1 stalk, which we have seen in our data. Experimentation using additional tRNA, thus, possible different interactions with the L1 stalk, would help to provide further information on the important of these interactions to translation.

\section{Understanding L1 stalk dynamics by rate analysis of the SmFRET data}

We examined the forward and reverse rate of L1 stalk motion to try to better understand the mechanism and dynamics of the L1 stalk. We compared the forward and reverse rates of the Wt L1/L33 construct to the mutant constructs: R53A, K54A, R60A, R164A and triple. The forward and reverse rates for the Wt and L1/L33 mutant constructs were examined when assembled with: tRNA-fMet, or tRNAfMet, Nac-phe-tRNA-Phe or Nac-phe-tRNA-Phe. The L1 proteins mutational sites were chosen to disrupt specific known interactions between the L1 protein and tRNA bound to the ribosome, specifically the L1 proteins interactions with $55 \psi$ of tRNA-Met [15]. The changes in the forward and reverse rate that we observed are thought to be the result of the specific mutations but may be due to number of effects like: general structural changes, altered binding sites, and the L1 stalks inability properly transition between states. The rate data from the mutant constructs suggests that the L1 stalks interaction with the tRNA bound to the ribosome change throughout translocation. We also found that the mutation sites we used had different effects on the L1 stalk dynamics that depended on what tRNA was assembled with the ribosome. There was a large amount of variability in the L1/L33 ribosome mutant constructs forward and reverse rates but they generally stayed within a factor of two of the L1/L33 Wt rate.

The interpretation of smFRET data assembled with a variety of different tRNA, examined in the context of crystallographic and CryoEM data, may help to further our understanding of the mechanism of translation. Our data is further supported by studies detailing the mutation of the $\psi 55$ residue of tRNA-fMet which has shown it to be the interacting partner of some of our L1 protein mutational sites [15]. 
The forward rates for the L1/L33 mutant constructs assembled with tRNA-fMet increased. The largest changes were for the forward rate, or closure of the L1 stalk, for the R60A and R164A mutant constructs which were $75 \%$ larger than the Wt. Also, the R53A, R54A and the triple mutant constructs increased about $40 \%$ over the Wt construct. The increase in forward rate is thought to be the result of the L1 proteins altered structure in response to the specific mutations and not due to its direct interaction with the tRNA (5.3.0 Table 9).

The ribosome constructs assembled with tRNA-fMet had larger reverse rate for all of the constructs except K54A which decreased but only by $10 \%$ of the Wt. This was attributed to the K54A residue being positioned differently when interacting with tRNA-fMet and in changes to the conformation of the L1 protein during interaction with tRNA-fMet.

For the majority of the forward and reverse rates for the L1/L33 mutant constructs assembled with tRNA-fMet their rates increased. The increase in the forward and reverse rates would suggest that these mutations resulted in a lowering of the energy barrier needed to transition between the open and closed states.

For the ribosomes mutant constructs assembled with tRNA-fMet and Nac-Phe-tRNA-Phe, the forward rate increased for the K54A and R60A, and it decreased for R53A, R164A and the triple. The data suggests that K54A and R60A lowered the energy barrier between the open and closed state while R53A, R164A and the triple constructs increased the energy barrier needed to transition to the closed state.

A small decrease in the reverse rate was seen for the K54A and triple constructs assembled with tRNA-fMet Nac-Phe-tRNA-Phe. A larger decrease, almost half of the Wt rate was seen for the R53A, R60A and R164A constructs. A decrease in the reverse rate would suggest that the L1 mutations strengthened the L1 proteins-tRNA interaction and increasing the energy need to transition to the open state.

The forward and reverse rates of the Wt L1/L33 construct increased when assembled with tRNAfMet, Nac-Phe-tRNA-Phe from tRNA-fMet. The reverse rate of R60A L1/L33 construct assembled with tRNA-fMet, Nac-Phe-tRNA-Phe dropped below the rate of the Wt L1/L33 construct when assembled with tRNA-fMet. The R60A L1/L33 mutant ribosome constructs reverse rate, when assembled with tRNA-fMet, was much higher than the same constructs assembled with tRNA-fMet, Nac-Phe-tRNA-Phe. Our data 
suggest that the R60A residue of the L1 protein may be critical to the interaction between the L1 protein and tRNA-fMet. The R164A L1/L33 mutant ribosome constructs reverse rate remained the same when assembled with tRNA-fMet or tRNA-fMet, Nac-Phe-tRNA-Phe. This leads us to believe that it may not be as important to binding of the tRNA except that the histograms of the smFRET data for this construct remain similar to the other mutants.

All of the L1/L33 mutant ribosome constructs assembled with Nac-Phe-tRNA-Phe had a decrease in their forward rate except K54A which increased. R164A L1/L33 mutant ribosome construct had a slight decrease while the triple almost decreased by half the Wt. An increase in the forward rate of only the K54A L1/L33 mutant ribosome construct is, as we mentioned previously, likely due to its position on the L1 proteins structure.

The reverse rates decreased for the L1/L33 mutant ribosome constructs, assembled with Nac-PhetRNA-Phe, except the triple, which had an increase of almost doubled the Wt. The R53A and K54A L1/L33 mutant ribosome constructs reverse rates decrease to halve of the Wt, while the R60A and R164A saw a much smaller decrease in their reverse rates. Single mutants, R60A and R164A look to have less of an effect on the forward and reverse rate of L1/L33 mutant ribosome constructs assembled with Nac-PhetRNA-Phe than the R53A, K54A and the triple mutants.

The data from the L1/L33single mutant ribosome constructs suggests that the L1 protein-tRNA interaction is much more complicated than previous thought but there are trends in the data which set specific residues apart from the rest. For the majority of the L1/L33mutant ribosome constructs tested, the reverse rate was larger than the forward rate except for L1/L33 mutant constructs R53A and K54A assembled with Nac-Phe-tRNA-Phe. We found this unusual except when you consider that only the fluctuating traces are used for the rate analysis. The proportion of non-fluctuating traces in either the open or closed state must change depending on the mutant ribosome construct in order for the mutant construct to have such similar histograms. Our data demonstrates that the mutational sites we chose do disrupt, which have been shown to interact with $\psi 55$ residue of tRNA-fMet, change the dynamics of the L1 stalk.

More experimental data is needed to further our understanding with this project. Additional smFRET data with a variety of tRNA combinations would help to sort out their unique interaction with the L1 stalk. This data would provide informative to the continued study of the ribosome with X-ray 
crystallography and CryoEM. A limitation of the data that we collected is the large amount of variability due to the small sample size with $80-429$ transitions per rate analysis. The results are within reason when compared to previously published values [4].

\section{Removal of the L1 protein results in the ribosome unable to form the rotated state}

Ribosomes lacking the L1 protein portion of the L1 stalk were unable to assume the rotated state even in circumstances where the ribosome would normally primarily occupy the rotated state. Wildtype S6/L9 ribosomes prepared with tRNA-fMet in the P-site had 77\% of the molecules occupy the rotated state

with $71 \%$ of the molecules having fluctuations. Ribosomal construct S6/L9/ $\mathrm{L} 1$ had molecules occupy the rotated state $41 \%$ of the time with $70 \%$ molecules containing at least one transition between the rotated and non-rotated states. Removal of the L1 stalk would remove its possible interaction with tRNA bound to the ribosomes and the interactions it provides to tRNA. Energy provided by the L1protein to stabilization of tRNA-fMet would be lost and a possible decrease in the rotated state stabilization would be consistent.

Wildtype S6/L9 ribosomes prepared with tRNA-fMet in the P-site and Nac-Phe-tRNA-Phe in the A site had $69 \%$ of the molecules occupy the rotated state and only $59 \%$ of molecules containing transitions. S6/L9/ $\mathrm{L} 1$ ribosomes had only $37 \%$ of molecules occupy the rotated state and $54 \%$ containing transition. The S6/L9/ $\mathrm{L} 1$ ribosomes spend significantly less time in the rotated state because of the ribosome lacks the L1 protein-tRNA interaction. This data shows that without the L1 stalk-tRNA interaction, the ribosome is unable to assume the Pre-translocation state which helps to explain the reduced growth phenotype of cells lacking the L1 protein from their genome.

Wildtype S6/L9 ribosomes prepared with Nac-Phe-tRNA-Phe in the P-site had molecules $25 \%$ of time in the rotated state with only $4 \%$ of molecules containing transitions. S6/L9/ $\Delta \mathrm{L} 1$ ribosomes had molecules occupy the rotated state $44 \%$ of the time with $50 \%$ containing transitions. Together this data indicates that the L1 protein component of the L1 stalk seems to be involved in the stabilization of the ribosome multiple processes during elongation. Ribosomes lacking the L1 stalk obtain a muted distribution of states and are unable to fully occupy the rotated or non-rotated state. This indicates the importance of the L1 stalk-tRNA interaction toward global dynamics of the ribosome.

\section{Investigation of subunit rotation with L1 stalk mutants}


Despite our current understanding of the various interacting partners of the ribosome there is still a substantial amount we do not know about how these interactions between multiple different mobile elements of the ribosome facilitates translation.

A set of constructs with identical mutational sites to the L1/L33 construct were generated to examine subunit rotation using S6/L9, however, because of difficulties with the method of construct generation, the ribosomes produced by this method were defective and not suitable. It is our thought that the generation of these constructs through an alternative approach would likely result in ribosomes of sufficient quality for smFRET studies that could examine subunit rotation with mutation to the L1 stalktRNA interface. Further investigation of the L1 stalk-tRNA interaction through observations of changes in subunit rotation as a result of $\mathrm{L} 1$ protein mutations would help to demonstrate the importance and interconnectivity of dynamics of the ribosome. Interactions between the L1 stalk and tRNA may be involved in the majority of ribosomal processes including ribosomal frameshifting or ribosomal pausing. Understanding how the thermodynamic landscape of the ribosome changes during translation and how various factors influence its processes would help to build a more detailed understanding of the ribosome.

\subsubsection{Conclusions}

Our findings demonstrate that disruption of the L1 stalk-tRNA interface alters the ability of the L1 stalk to form both the open and closed conformation. These findings also show that removal of the L1 portion of the L1 stalk results in ribosomes that are unable to correctly form the rotated state during intersubunit rotation. Rate analysis yielded results that were comparable to published values and demonstrated that specific mutations alter the dynamics of the ribosome differently during translation. Our results are consistent with proposed hypotheses and represent an important method for continued investigation into the importance of the L1 stalk-tRNA interface. Our hope is that by better understanding the ribosome dynamics and the mechanism of protein synthesis our results will help in the development of treatment for diseases and possibly be used as a starting point for the investigation of eukaryotes.

\subsubsection{Acknowledgements}

This work was supported by National Science Foundation CAREER award [grant number: MCB115343] to P.V.C. P.V.C is a Pew Scholar in the Biomedical Sciences. 


\subsubsection{Contributions}

Drew Menke was responsible for the entire project.

\subsubsection{References}

1. Cornish, P.V., et al., Spontaneous Intersubunit Rotation in Single Ribosomes. Molecular Cell, 2008. 30(5): p. 578-588.

2. Horan, L.H. and H.F. Noller, Intersubunit movement is required for ribosomal translocation. Proceedings of the National Academy of Sciences of the United States of America, 2007. 104(12): p. 4881-4885.

3. Mohan, S. and H.F. Noller, Recurring RNA structural motifs underlie the mechanics of L1 stalk movement. Nature Communications, 2017. 8: p. 14285.

4. Cornish, P.V., et al., Following movement of the L1 stalk between three functional states in single ribosomes. Proceedings of the National Academy of Sciences, 2009. 106(8): p. 2571-2576.

5. Fei, J., et al., Allosteric collaboration between elongation factor G and the ribosomal L1 stalk directs tRNA movements during translation. Proceedings of the National Academy of Sciences of the United States of America, 2009. 106(37): p. 15702-15707.

6. Blanchard, S.C., et al., tRNA selection and kinetic proofreading in translation. Nat Struct Mol Biol, 2004. 11(10): p. 1008-1014.

7. Blanchard, S.C., et al., tRNA dynamics on the ribosome during translation. Proceedings of the National Academy of Sciences of the United States of America, 2004. 101(35): p. 12893-12898.

8. Lee, T.-H., et al., The role of fluctuations in tRNA selection by the ribosome. Proceedings of the National Academy of Sciences, 2007. 104(34): p. 13661-13665.

9. Kim, H.D., J.D. Puglisi, and S. Chu, Fluctuations of Transfer RNAs between Classical and Hybrid States. Biophysical Journal, 2007. 93(10): p. 3575-3582.

10. Fei, J., et al., Transfer RNA-mediated regulation of ribosome dynamics during protein synthesis. Nat Struct Mol Biol, 2011. 18(9): p. 1043-1051.

11. Salsi, E., et al., Following movement of domain IV of elongation factor G during ribosomal translocation. Proceedings of the National Academy of Sciences of the United States of America, 2014. 111(42): p. 15060-15065. 
12. Salsi, E., et al., Movement of Elongation Factor G between Compact and Extended Conformations. Journal of molecular biology, 2015. 427(2): p. 454-467.

13. Munro, J.B., et al., Spontaneous formation of the unlocked state of the ribosome is a multistep process. Proceedings of the National Academy of Sciences of the United States of America, 2010. 107(2): p. 709-714.

14. Munro, J.B., et al., A fast dynamic mode of the EF-G-bound ribosome. The EMBO Journal, 2010. 29(4): p. 770-781.

15. Bock, L.V., et al., Energy barriers and driving forces in tRNA translocation through the ribosome. Nat Struct Mol Biol, 2013. 20(12): p. 1390-1396.

16. Munro, J.B., et al., Identification of Two Distinct Hybrid State Intermediates On the Ribosome. Molecular cell, 2007. 25(4): p. 505-517.

17. Réblová, K., J. Šponer, and F. Lankaš, Structure and mechanical properties of the ribosomal L1 stalk three-way junction. Nucleic Acids Research, 2012. 40(13): p. 6290-6303.

18. Qin, P., et al., Structured mRNA induces the ribosome into a hyper-rotated state. EMBO Reports, 2014. 15(2): p. 185-190.

19. Ling, C. and D.N. Ermolenko, Initiation factor 2 stabilizes the ribosome in a semirotated conformation. Proceedings of the National Academy of Sciences of the United States of America, 2015. 112(52): p. 15874-15879.

20. Datsenko, K.A. and B.L. Wanner, One-step inactivation of chromosomal genes in Escherichia coli K-12 using PCR products. Proceedings of the National Academy of Sciences of the United States of America, 2000. 97(12): p. 6640-6645.

21. Blanco, M. and N. Walter, Analysis of Complex Single Molecule FRET Time Trajectories. Methods in enzymology, 2010. 472: p. 153-178.

22. Schuster-Böckler, B. and A. Bateman, An Introduction to Hidden Markov Models, in Current Protocols in Bioinformatics. 2002, John Wiley \& Sons, Inc.

23. McKinney, S.A., C. Joo, and T. Ha, Analysis of Single-Molecule FRET Trajectories Using Hidden Markov Modeling. Biophysical Journal, 2006. 91(5): p. 1941-1951. 
24. Laursen, B.S., et al., Initiation of Protein Synthesis in Bacteria. Microbiology and Molecular Biology Reviews, 2005. 69(1): p. 101-123. 


\subsubsection{Appendix}

\subsubsection{Patent}

(19) United States

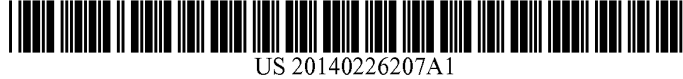

(12) Patent Application Publication

(10) Pub. No.: US 2014/0226207 A1 Gangopadhyay et al.

(43) Pub. Date: Aug. 14, 2014

(54) NANO-GAP GRATING DEVICES WITH ENHANCED OPTICAL PROPERTIES AND METHODS OF FABRICATION

(22) Filed: Nov. 15, 2013

Related U.S. Application Data

(71) Applicant: The Curators of the University of Missouri, Columbia, MO (US)

(60) Provisional application No. 61/850,232, filed on Feb. 11, 2013.

Publication Classification

(72) Inventors: Shubhra Gangopadhyay, Columbia, MO (US); Venu Korampally, Aurora, I (US); Sagnik Basuray, Columbia, MO (US); Kunal Bhatnagar, College Station, TX (US); Avinash Pathak, Columbia, MO (US); Arnab Ghosh, Columbia, MO (US); Drew Edwin Menke, Berger, MO (US); Joseph Menke, Berger, MO (US); Joseph
Mathai, Columbia, MO (US); Peter Cornish, Columbia, MO (US); Keshab Gangopadhyay, Columbia, MO (US); Aaron Wood, Columbia, MO (US)

(73) Assignee: THE CURATORS OF THE UNIVERSITY OF MISSOURI Columbia, MO (US)

(51) Int. Cl. G02B 5/18 (2006.01)

(52) U.S. Cl. CPC …….... G02B 5/1842 (2013.01); G02B 5/1852 USPC …................................. 359/566; $101 / 483$ (57) ABSTRACT

A method of producing a grating structure comprises the steps of forming a stamp from flexible plastic material, the stamp including a negative of a periodic grating pattern on a first surface; forming an ink by applying a polymer film to the first strface, forming an ink by applying a polymer film to the stamp, the ink including a first surface and an opposing second surface, wherein the first surface of the ink contacts the first surface of the stamp such that the ink retains a positive of the periodic grating pattern; placing the ink and the stamp on a substrate such that the second surface of the ink contacts an upper surface of the substrate; and removing the stamp from

(21) Appl. No.: 14/081,353

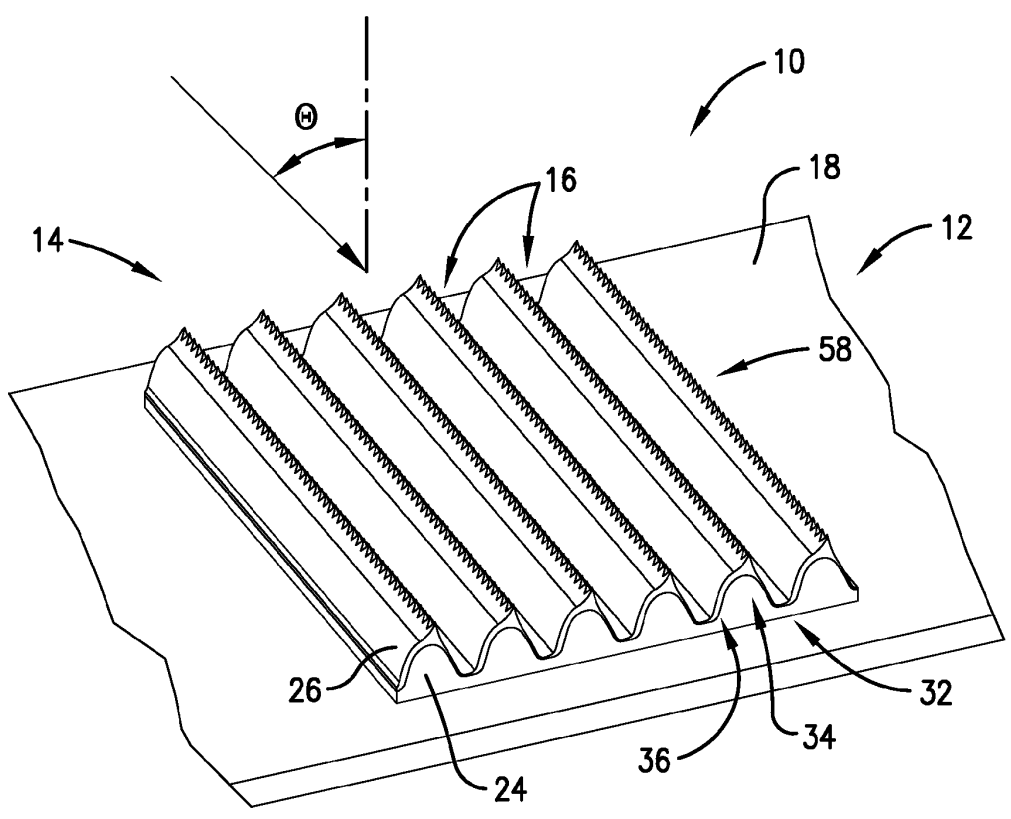


Patent Application Publication Aug. 14, 2014 Sheet 1 of 12 US 2014/0226207 A1
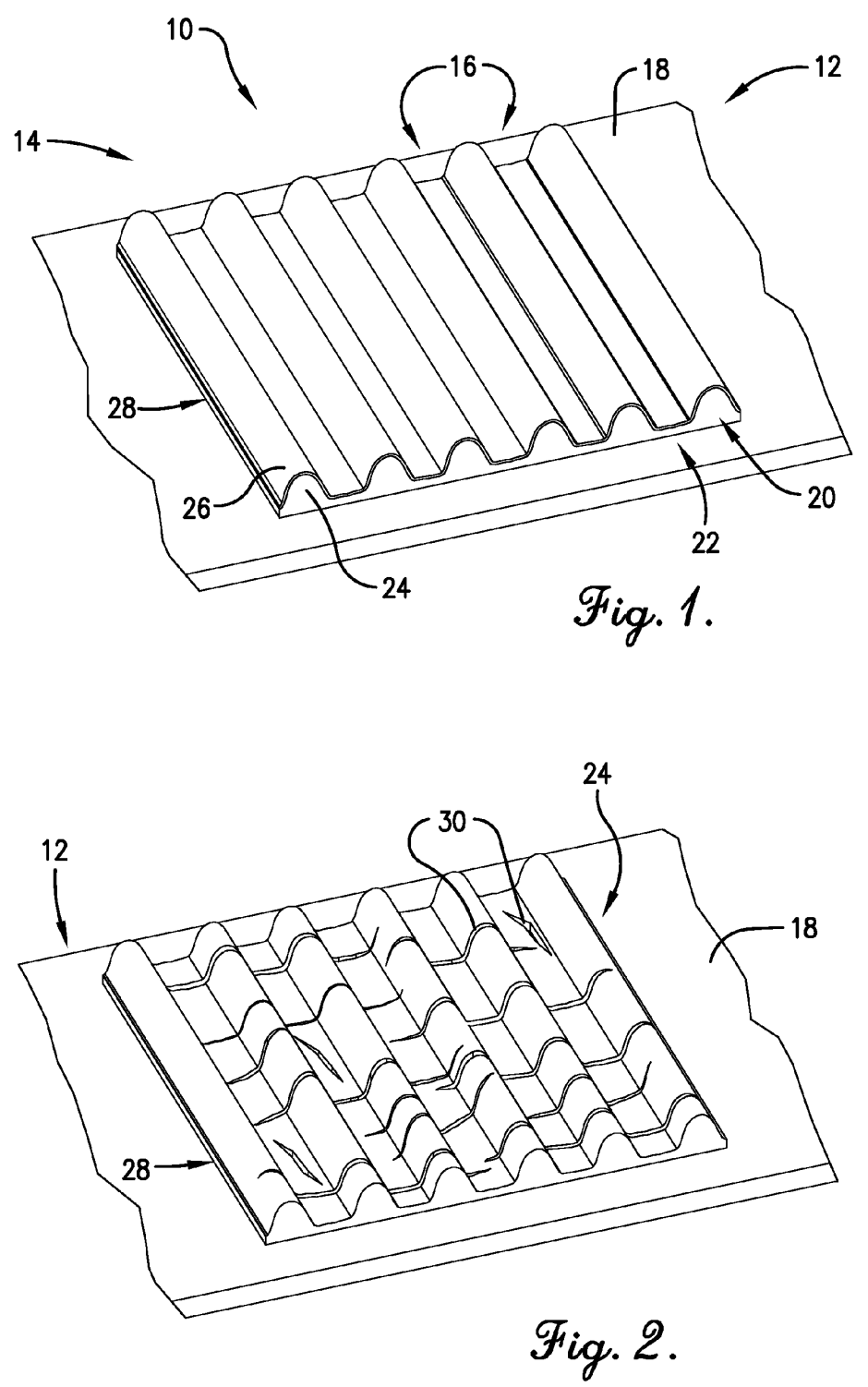
Patent Application Publication Aug. 14, 2014 Sheet 2 of 12 US 2014/0226207 A1
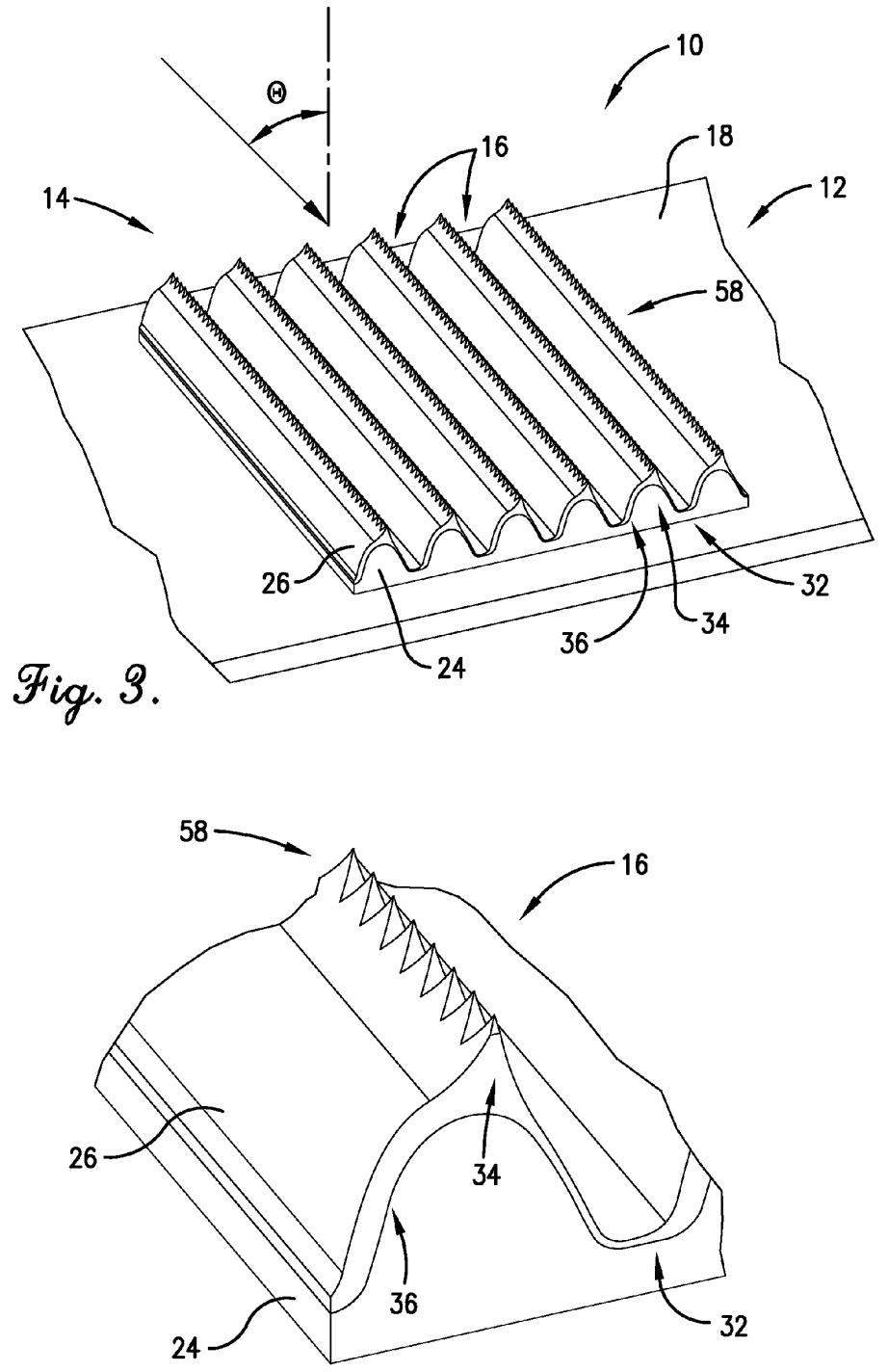

Fig. 4. 
Patent Application Publication Aug. 14, 2014 Sheet 3 of 12 US 2014/0226207 A1

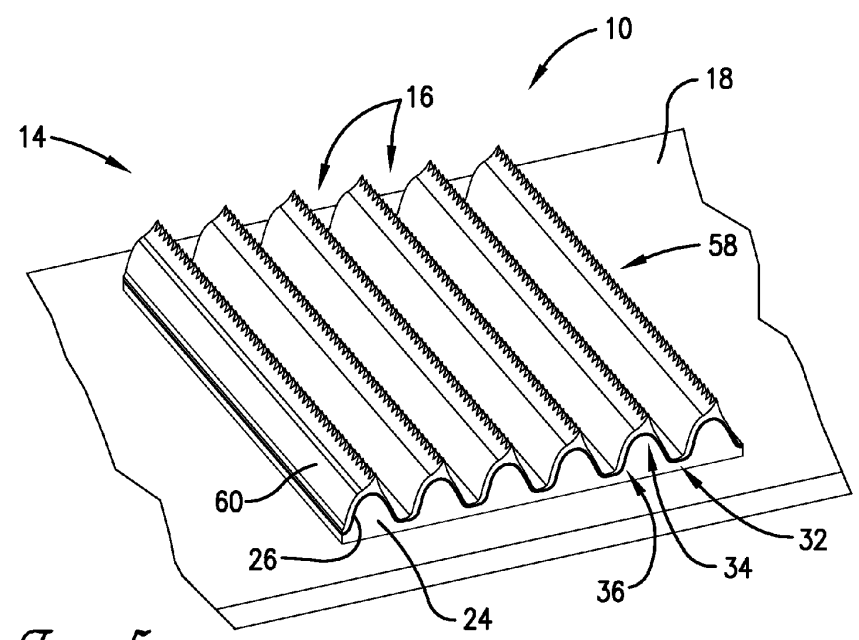

Fig. 5.

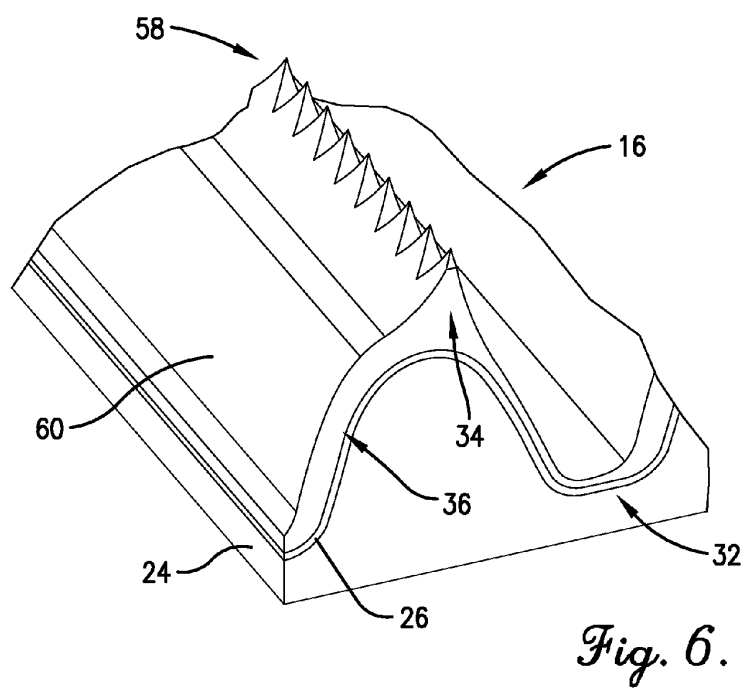


100

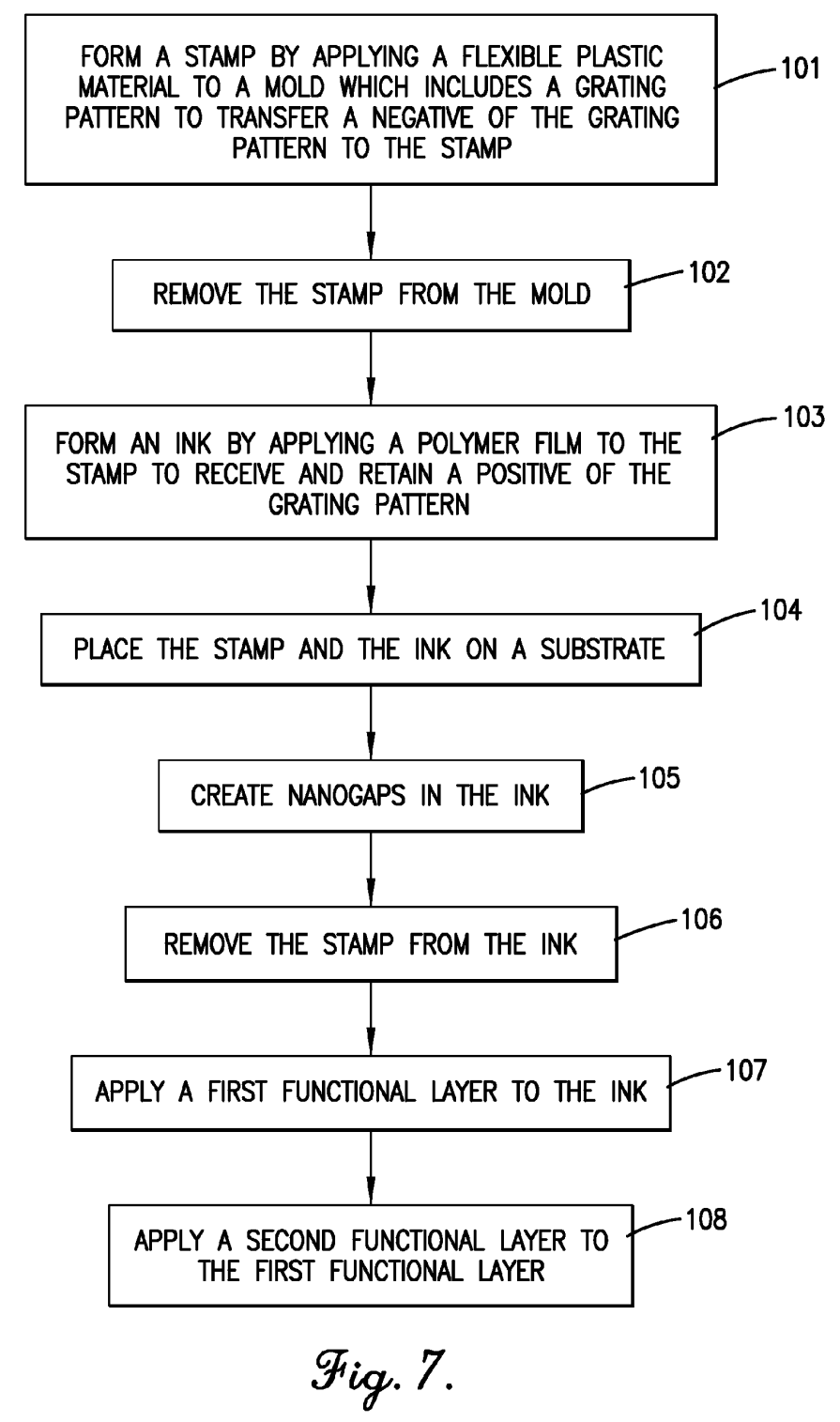


Patent Application Publication Aug. 14, 2014 Sheet 5 of 12 US 2014/0226207 A1

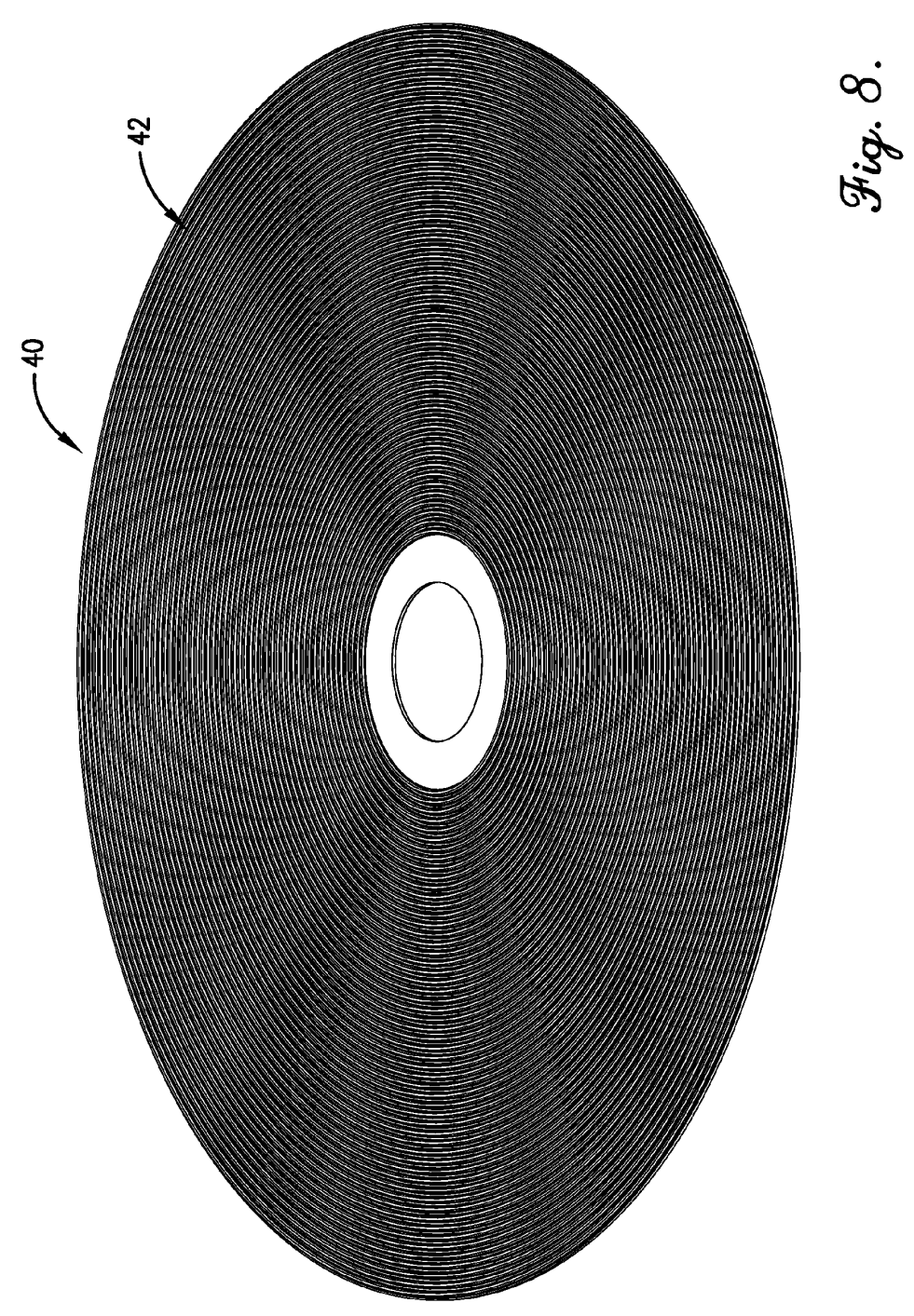


Patent Application Publication Aug. 14, 2014 Sheet 6 of 12 US 2014/0226207 A1

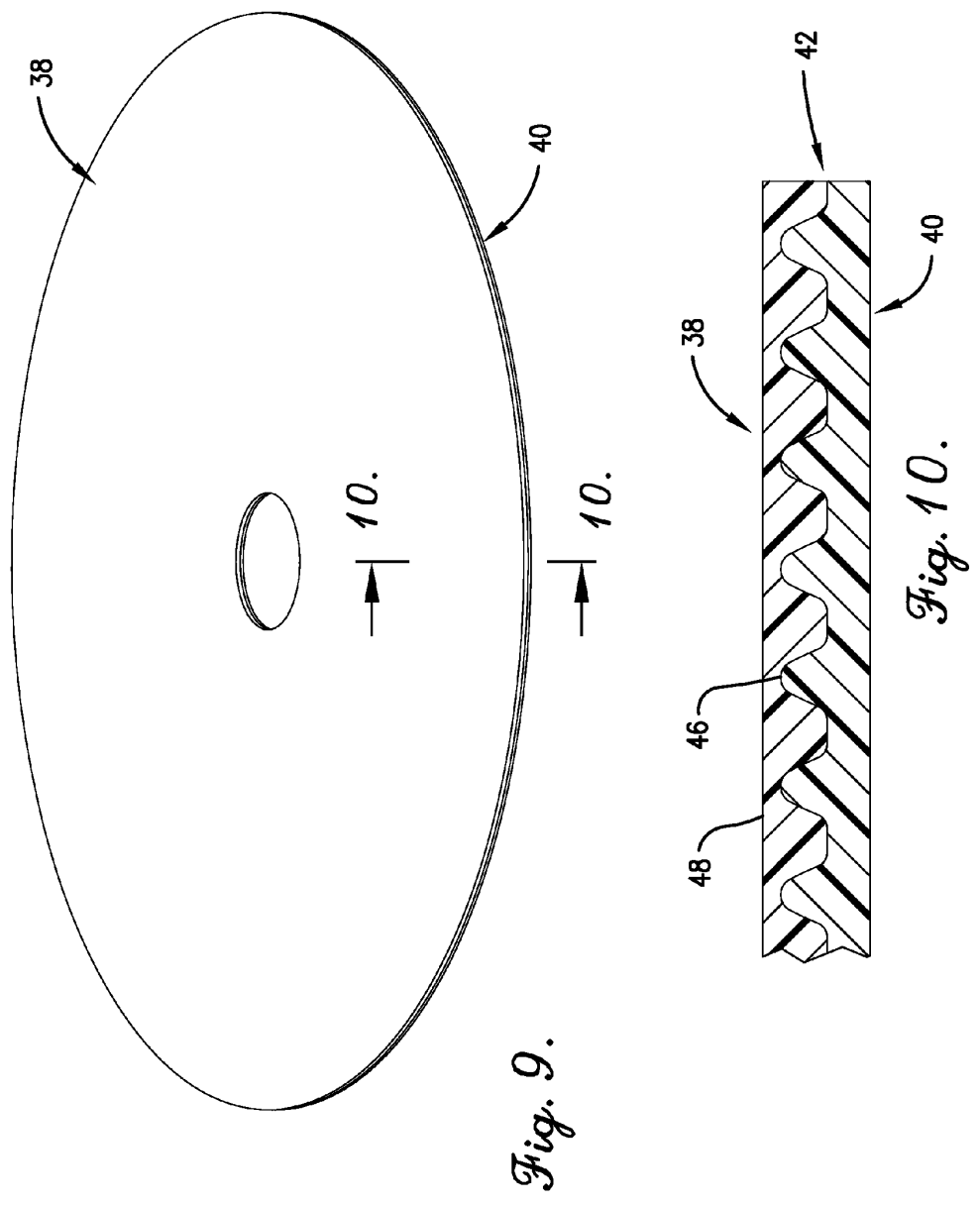


Patent Application Publication Aug. 14, 2014 Sheet 7 of 12

US 2014/0226207 A1

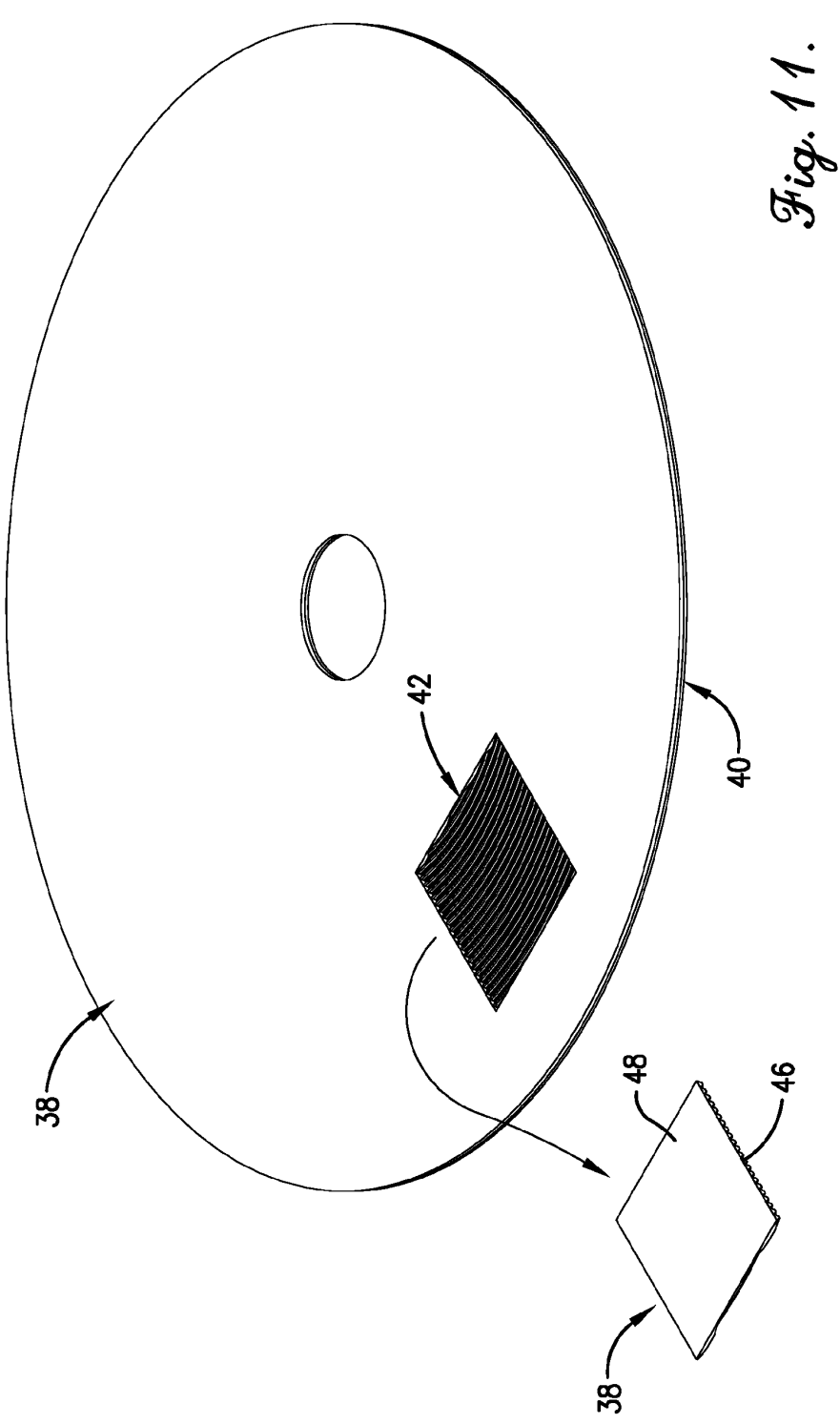


Patent Application Publication Aug. 14, 2014 Sheet 8 of 12 US 2014/0226207 A1
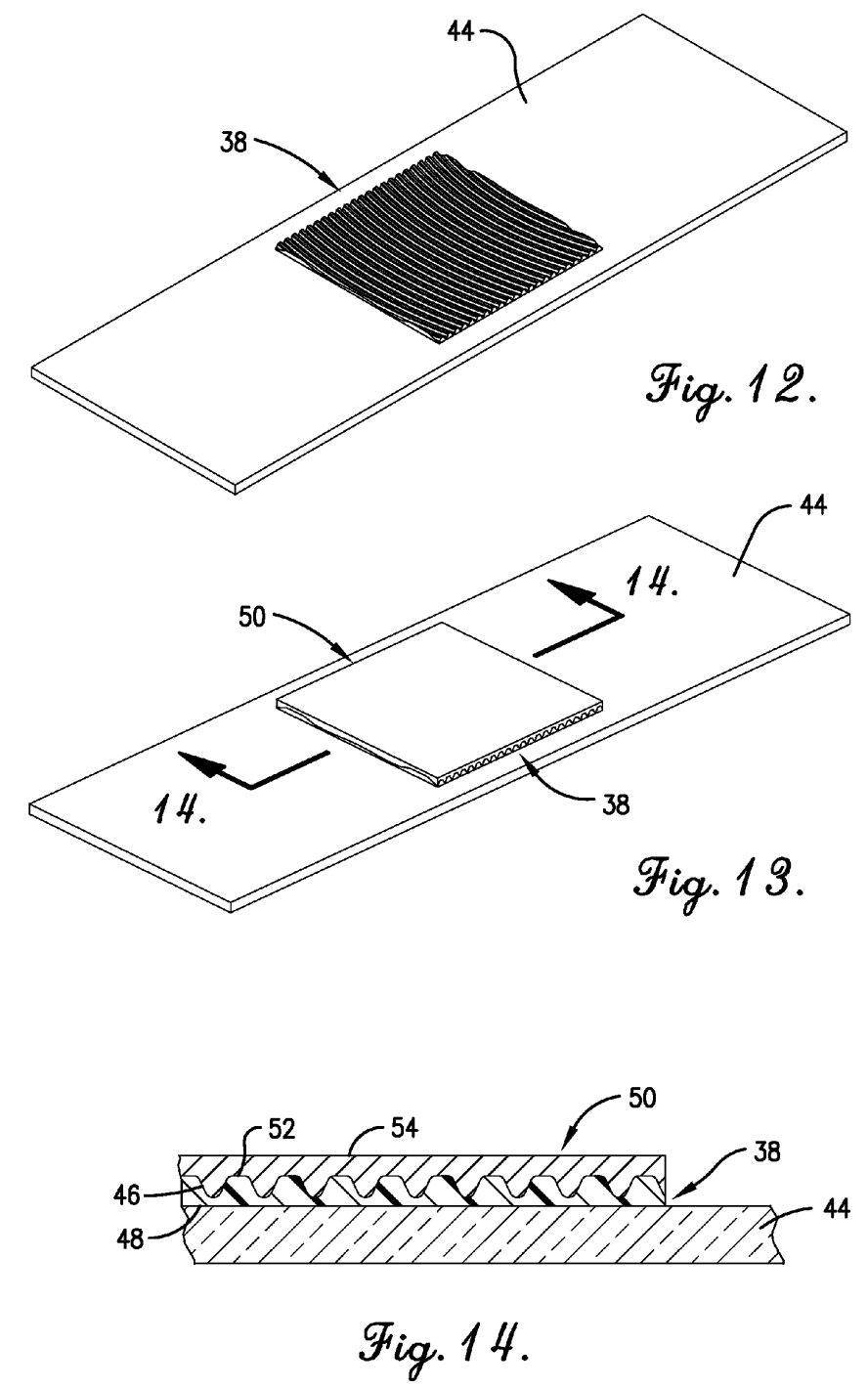
Patent Application Publication Aug. 14, 2014 Sheet 9 of 12 US 2014/0226207 A1
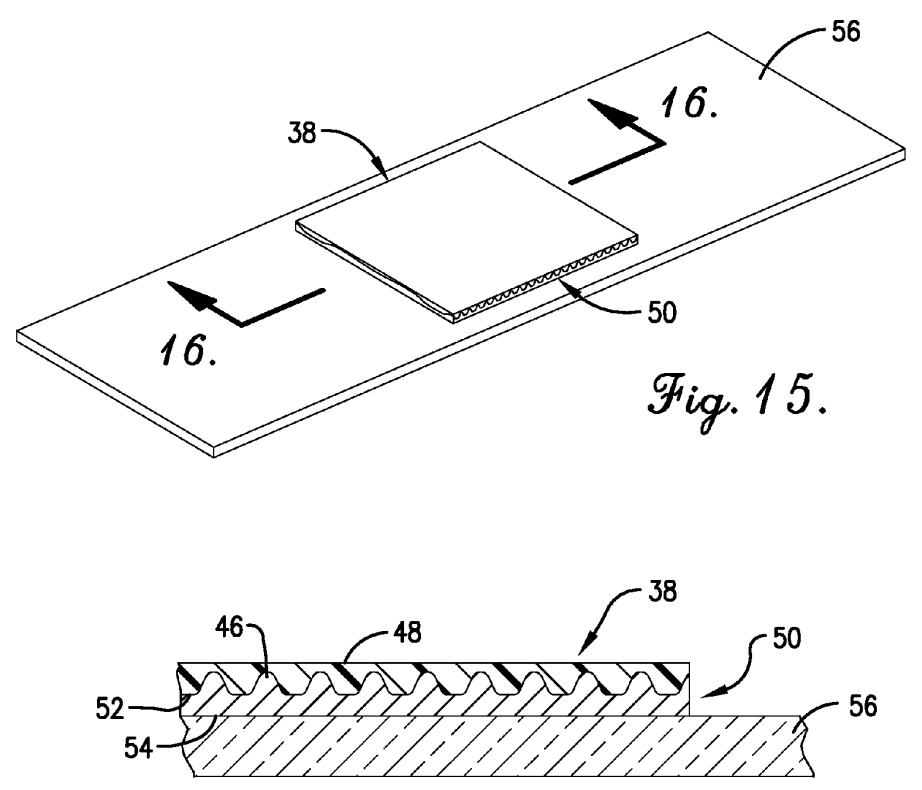

Fig. 16 .

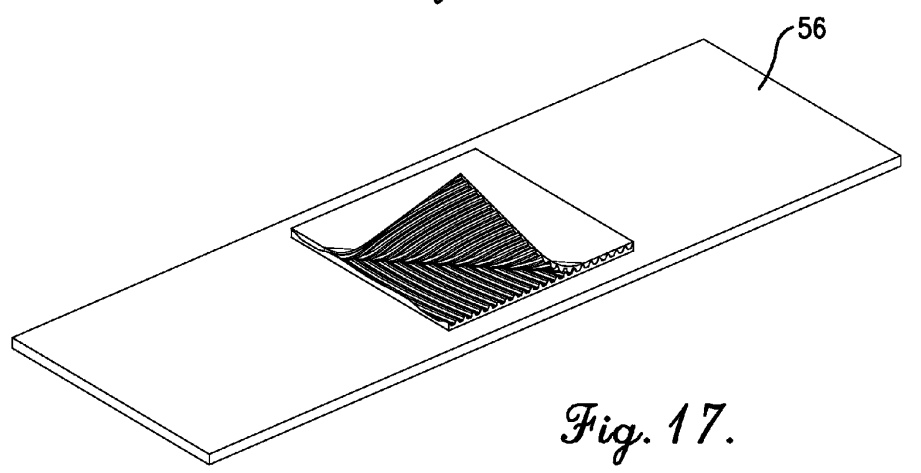


$\underline{200}$

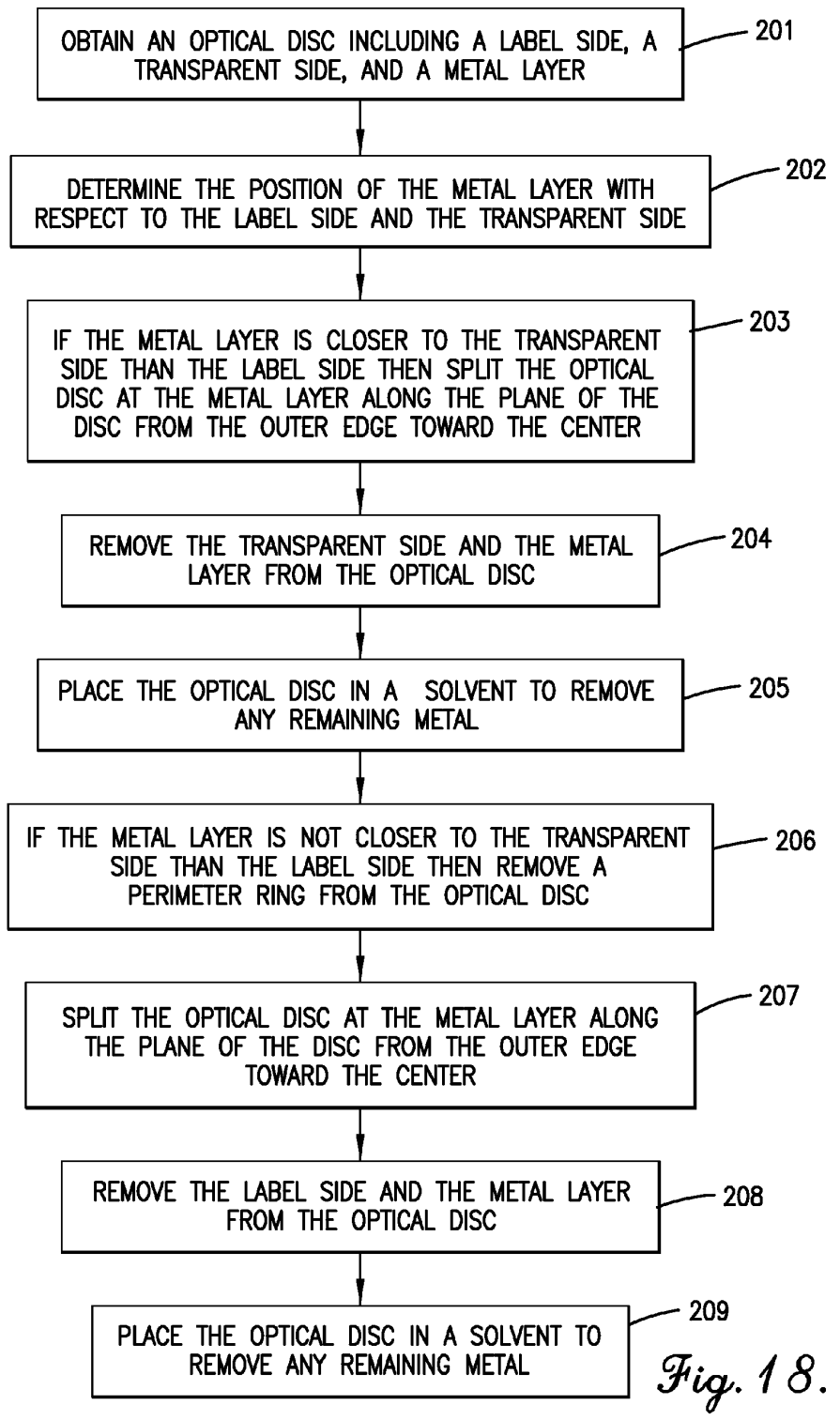



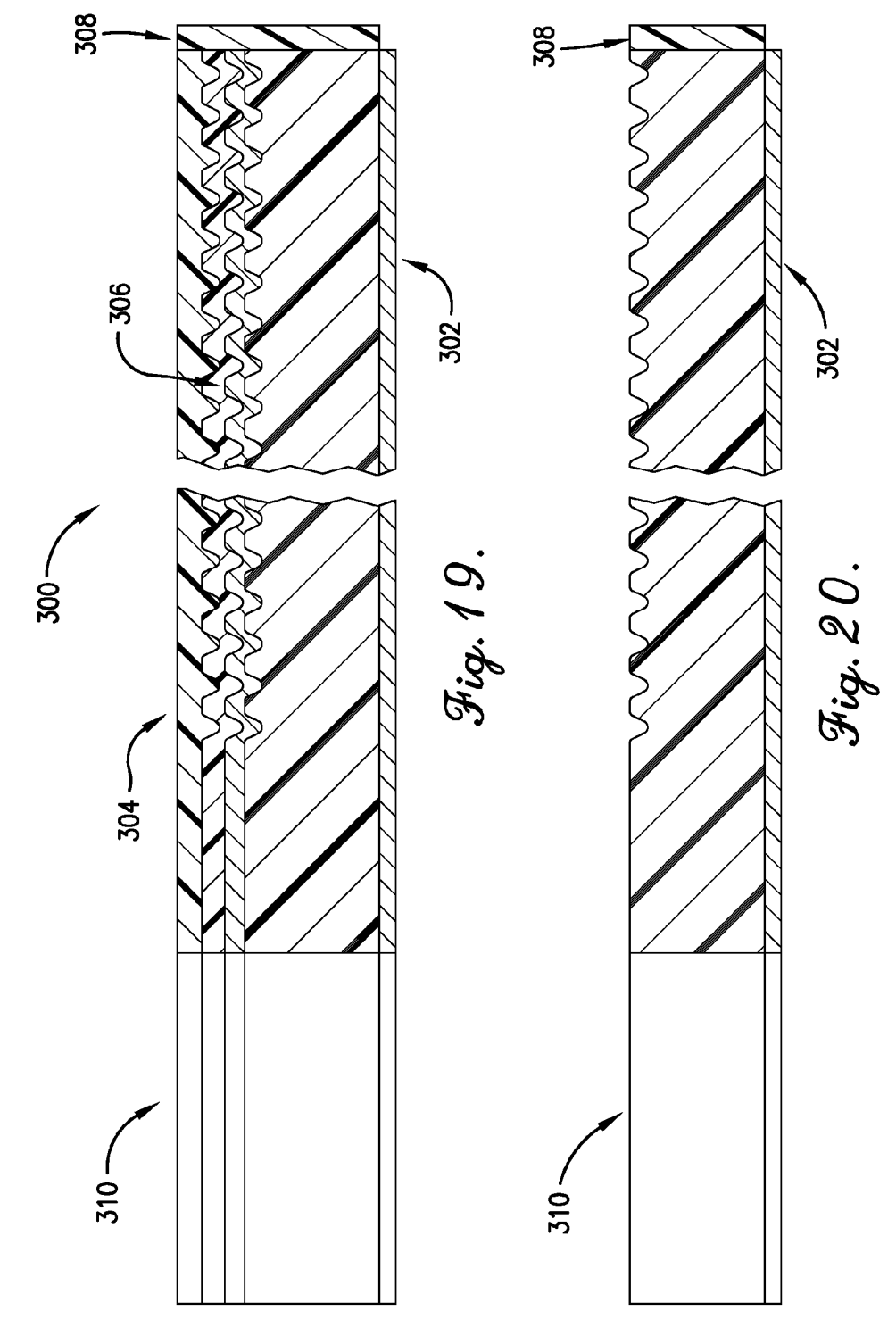
Patent Application Publication Aug. 14, 2014 Sheet 12 of 12 US 2014/0226207 A1

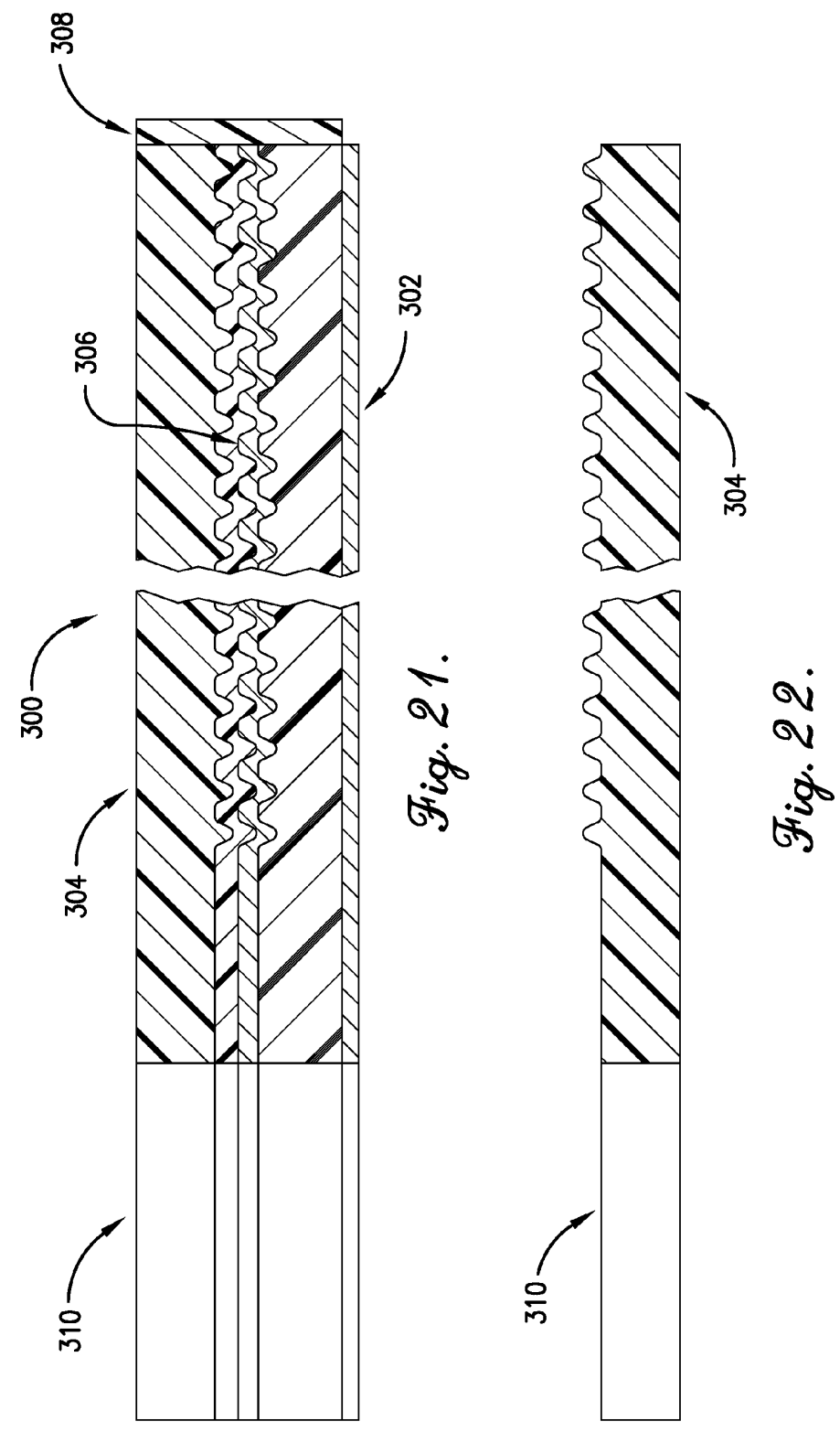




\section{NANO-GAP GRATING DEVICES WITH ENHANCED OPTICAL PROPERTIES AND METHODS OF FABRICATION}

\section{RELATED APPLICATION}

[0001] The present application is a non-provisional application, and claims priority benefit with regard to all commo subject matter, of U.S. Provisional Application No. 61/850, 232, filed Feb. 11, 2013, and entitled "NANO-GAP GRAT232, filed Feb. 11, 2013, and entitled "NANO-GAP GRATING DEVICES WITH ENHANCED OPTICAL PROP-
ERTY AND FABRICATIONS THEREOF." The identified ERTY AND FABRICATIONS THEREOF." The identified
earlier-filed provisional application is hereby incorporated by reference in its entirety.

\section{FEDERALLY SPONSORED RESEARCH OR} DEVELOPMENT

[0002] This invention was made with Government support under Grant No. ECCS-1102070 awarded by the National Science Foundation and Grant No. W15QKN-11-9-0001RPP1-H awarded by the Nano Technology Enterprise Consortium (NTEC). The Government has certain rights in the invention.

\section{BACKGROUND}

[0003] 1. Field

[0004] Embodiments of the invention relate to structures for improving optical imaging and methods of their fabrication.

[0005] 2. Related Art

[0006] Microscopic imaging is utilized for studying small objects in fields such as medical development, biological research, cancer research, metallurgy, and others. Imaging of microscopic objects often encounters the diffraction limit when trying to image increasingly small objects. Fluorescence spectroscopy can be utilized in ultrasensitive chemical and biological threat sensors. But fluorescence spectroscopy suffers from low image contrast and a limit on the detection capabilities. Uniform, periodic grating structures have been developed to offer improvement in microscopic imaging and fluorescence spectroscopy. However, the improvements may be limited and the production of the grating structures may require the usage of costly fabrication equipment and complex manufacturing techniques, leading to a high cost of the grating structures.

\section{SUMMARY}

[0007] Embodiments of the invention solve the above-mentioned problems and provide a distinct advance in the art of microscopic imaging and fluorescence spectroscopy. More particularly, embodiments of the invention provide grating structures with enhanced optical properties and methods of their fabrication.

[0008] A first embodiment of the invention provides a grating structure comprising a substrate, a base layer, and a first functional layer. The base layer is positioned on the substrate and includes a first surface with a plurality of grating elements positioned adjacent one another and an opposing second surface in contact with a surface of the substrate. The grating elements include a longitudinal peak and a longitudinal valley. The functional layer is positioned on the second surface of the base layer and provides electromagnetic field enhancement in the vicinity of the grating structure.
[0009] A second embodiment of the invention provides a method of producing a grating structure. The method comprises the steps of forming a stamp from flexible plastic material, the stamp including a negative of a periodic grating pattern on a first surface; forming an ink by applying a polymer film to the stamp, the ink including a first surface and an mer film to the stamp, the ink including a first surface and an
opposing second surface, wherein the first surface of the ink contacts the first surface of the stamp such that the ink retains a positive of the periodic grating pattern; placing the ink and the stamp on a substrate such that the second surface of the ink contacts an upper surface of the substrate; and removing the stamp from the ink by applying a tensional force to one edge of the stamp.

[0010] A third embodiment of the invention provides a method of producing a. The method comprises the steps of forming a stamp by applying a flexible plastic material to a mold which includes a periodic grating pattern, wherein the stamp retains a negative of the grating pattern on a first surface; forming an ink by applying a polymer film to the stamp, the ink including a first surface and an opposing second surface, wherein the first surface of the ink contacts the first surface of the stamp such that the ink retains a positive of the periodic grating pattern; placing the ink and the stamp on a substrate such that the second surface of the ink contacts an upper surface of the substrate; removing the stamp from the ink by applying a tensional force to one edge of the stamp; and applying a functional layer to the first surface of the ink.

[0011] This summary is provided to introduce a selection of concepts in a simplified form that are further described below in the detailed description. This summary is not intended to identify key features or essential features of the claimed subject matter, nor is it intended to be used to limit the scope of the claimed subject matter. Other aspects and advantages of the invention will be apparent from the following detailed description of the embodiments and the accompanying drawing figures.

BRIEF DESCRIPTION OF THE DRAWING FIGURES

[0012] Embodiments of the invention are described in detail below with reference to the attached drawing figures, wherein:

[0013] FIG. 1 is a perspective view of a grating structure including a first functional layer, a base layer, and a substrate, constructed in accordance with various embodiments of the invention;

[0014] FIG. 2 is a perspective view of the base layer and the substrate from the grating structure of FIG. 1, the base layer including a plurality of nanogaps

[0015] FIG. 3 is a perspective view of the grating structure with the first functional layer being deposited at an angle away from normal to the plane of the grating structure;

[0016] FIG. 4 is an enlarged view of a portion of the grating structure of FIG. $\mathbf{3}$

[0017] FIG. 5 is a perspective view of the grating structure with the first functional layer being deposited normal to the plane of the grating structure and a second functional layer being deposited at an angle away from normal to the plane of the grating structure;

[0018] FIG. 6 is an enlarged view of a portion of the grating structure of FIG. 5

[0019] FIG. 7 is a flow diagram of at least a portion of the steps of a method of producing a grating structure; 
[0020] FIG. 8 is a perspective view of a mold created from a modified optical disc;

[0021] FIG. 9 is a perspective view of the mold coated with a plastic material to form a stamp;

[0022] FIG. 10 is a sectional view of the mold and the stamp from FIG. 9 cut along the line $\mathbf{1 0 - 1 0}$

[0023] FIG. 11 is a perspective view of the mold and the stamp with a portion of the stamp removed to create a small stamp;

[0024] FIG. 12 is a perspective view of the stamp placed on a temporary substrate;

[0025] FIG. 13 is a perspective view of the stamp on the temporary substrate coated with a polymer film to create an ink;

[0026] FIG. 14 is a sectional view of the stamp and the ink from FIG. 13 cut along the line 14-14;

[0027] FIG. 15 is a perspective view of the stamp and the ink placed on a permanent substrate;

[0028] FIG. 16 is a sectional view of the stamp and the ink from FIG. 15 cut along the line 16-16;

[0029] FIG. 17 is a perspective view of the stamp and the ink on the permanent substrate depicting the stamp being removed from the ink

[0030] FIG. 18 is a flow diagram of at least a portion of the steps of a method of producing a mold that includes a grating structure;

[0031] FIG. 19 is a sectional view of a first type of optical disc with a metal layer and a transparent side in which the metal layer is closer to the transparent side;

[0032] FIG. 20 is a sectional view of the first type of optical [0032] FIG. 20 is a sectional view of the first type of optical
disc in which the metal layer and the transparent side have been removed

[0033] FIG. 21 is a sectional view of a second type of optical disc with a metal layer, a transparent side, and a label side in which the metal layer is not closer to the transparent side; and

[0034] FIG. 22 is a sectional view of the second type of optical disc in which the metal layer and the label side have been removed.

[0035] The drawing figures do not limit the invention to the specific embodiments disclosed and described herein. The drawings are not necessarily to scale, emphasis instead being placed upon clearly illustrating the principles of the invention.

\section{DETAILED DESCRIPTION}

[0036] The following detailed description of embodiments of the invention references the accompanying drawings that illustrate specific embodiments in which the invention can be illustrate specific embodiments in which the invention can be
practiced. The embodiments are intended to describe aspects practiced. The embodiments are intended to describe aspects
of the invention in sufficient detail to enable those skilled in of the invention in sufficient detail to enable those skilled in
the art to practice the invention. Other embodiments can be utilized and changes can be made without departing from the scope of the present invention. The following detailed description is, therefore, not to be taken in a limiting sense. The scope of the present invention is defined only by the appended claims, along with the full scope of equivalents to which such claims are entitled.

[0037] In this description, references to "one embodiment", "an embodiment", or "embodiments" mean that the feature or features being referred to are included in at least one embodiment of the technology. Separate references to "one embodiment" "an embodiment", or "embodiments" in this description do not necessarily refer to the same embodiment and are also not mutually exclusive unless so stated and/or except as will be readily apparent to those skilled in the art from the description. For example, a feature, structure, act, etc. described in one embodiment may also be included in other embodiments, but is not necessarily included. Thus, the technology can include a variety of combinations and/or integrations of the embodiments described herein.

[0038] A grating structure 10 with nanogap features, constructed in accordance with various embodiments of the invention, is shown in FIGS. 1, 3, and $\mathbf{4}$ and may broadly comprise a substrate $\mathbf{1 2}$ and an array $\mathbf{1 4}$ of grating elements 16. Various embodiments of the invention can provide optical wave guiding and imaging beyond the diffraction limit. The invention may be utilized in a sensing or imaging system which may improve the detection limits of harmful biological and chemical agents in food down to pico or femtomolar concentration levels. Similarly the invention may be used in anti-terrorism to detect any trace quantities of bio or chemically hazardous material. Embodiments of the invention may also be utilized in studying single molecule fluorescence phenomena in real time, enhancing surface-enhanced Raman spectroscopy (SERS), and the like.

[0039] The substrate 12, as shown in FIGS. 1-3, generally provides a structural base or foundational support for the grating elements 16 and may be formed from silicon, glass, or combinations thereof. The substrate $\mathbf{1 2}$ may include an upper surface 18 that receives the grating elements $\mathbf{1 6}$. The upper surface 18 may be planar and smooth so as to easily bond with the grating elements 16 .

[0040] The array 14, as shown in FIGS. 1 and 3 , is arranged on the substrate 12 so that the grating elements 16 are paralle and positioned abutting one another. Each grating element 16 is generally elongated, relatively narrow, and is formed with a longitudinal peak 20 positioned next to a longitudinal valley 22. In some embodiments, the longitudinal axis of the grating elements 16 may be straight, while in other embodiments, the longitudinal axis may be curved. In some embodiments, the peak 20 may have a constant and continuous height along the length of the grating element 16, while in other embodiments, the peak 20 may have a variable height along the length of the grating element 16

[0041] An exemplary grating element 16, as seen in FIG. 1, may have a cross-sectional profile that is a roughly half sine wave with the peak 20 having a generally rounded shape and the valley 22 being generally flat. An exemplary grating element $\mathbf{1 6}$ may have a width or pitch of approximately 400 nanometers $(\mathrm{nm})$. The width of each grating element 16 may vary depending on the application for which the orating structure $\mathbf{1 0}$ is used. An exemplary peak $\mathbf{2 0}$ may have a height relative to the valley 22 of approximately $60 \mathrm{~nm}$. In other embodiments, the grating element 16 may have a cross-sectional profile that is a roughly sine wave shape, a roughly triangle wave shape, a roughly square wave shape, a roughly sawtooth shape, variations thereof, or combinations thereof. [0042] Each grating element 16 may include a base layer 24 and a first functional layer 26. The base layer $\mathbf{2 4}$, as seen without the first functional layer $\mathbf{2 6}$ in FIG. 2, may be positioned on the substrate 12 and may include a lower surface 28 which contacts and adheres to the upper surface 18 of the substrate 12. The base layer $\mathbf{2 4}$ may be formed from a polymer material or a polymer film such as polymethylsilsesquioxane (PMSSQ) and may have the same cross-sectional profile as the grating element 16 including the peak 20 and the valley 22 . The base layer $\mathbf{2 4}$ may also be considered an ink. 
[0043] The base layer 24 may further include a plurality of nanogaps 30, best seen in FIG. 2, wherein each nanogap 30 is an elongated, generally narrow gap or opening in the material of the base layer 24. Exemplary nanogaps 30 may have a width ranging from approximately $20 \mathrm{~nm}$ to approximately $30 \mathrm{~nm}$, while the length of the nanogaps 30 may vary more greatly. In some embodiments, the nanogaps 30 may be randomly oriented and randomly distributed primarily in proximity to the edges of the array $\mathbf{1 4}$. In other embodiments, the nanogaps $\mathbf{3 0}$ may be more uniformly distributed across the array 14. In addition, the nanogaps 30 may generally be oriented or aligned with one another In still other embodiments, there may be a combination of randomly oriented and distributed nanogaps $\mathbf{3 0}$ and more uniformly oriented and distributed nanogaps $\mathbf{3 0}$.

[0044] The first functional layer 26, as seen in FIGS. 1, 3, and $\mathbf{4}$, generally provides electromagnetic field enhancement in the vicinity of the grating structure 10. The first functional in the fill in or completely cover the nanogaps 30 of the base layer $\mathbf{2 4}$. The first functional layer $\mathbf{2 6}$ may include either a plasmonic component or a photonic component. In embodiments of the first functional layer 26 with plasmonics, the first functional layer 26 primarily includes one or more layers of metal. An optional lower metal layer may provide improved adhesion between the base layer $\mathbf{2 4}$ and an upper layer of metal. An exemplary lower metal ayer may titanium, approximately $2 \mathrm{~nm}$ thick. A main upper layer of metal may provide plasmonic functionality. An exemplary metal for the upper metal layer is silver, approximately 100 $\mathrm{nm}$ thick, deposited on the first lower layer of metal. Silver is often utilized as the plasmonic material because it may allow or promote fluorescence in various applications of the grating structure 10 to a greater extent than other metals. In addition, silver may have a lower energy loss then other metals. Other metals can be used for either the first lower adhesion layer or the second, upper metal layer including silver, titanium, gold, chromium, alloys, or combinations thereof. In some embodiments, the plasmonic first functional layer $\mathbf{2 6}$ may further include a thin layer of dielectric or insulating material, such as silicon dioxide, rhodamine-doped PMSSQ, or similar materials positioned on the metal. The layer of dielectric may have a thickness ranging from approximately $10 \mathrm{~nm}$ to approximately $30 \mathrm{~nm}$.

[0045] In embodiments of the first functional layer 26 with photonics (also referred to as photonic crystals), the first functional layer $\mathbf{2 6}$ primarily includes one or more layers of dielectrics. An exemplary photonic first functional layer may include titanium oxide $\left(\mathrm{TiO}_{2}\right.$ ), with a approximately $100 \mathrm{~nm}$ to approximately $200 \mathrm{~nm}$, deposited on the base layer 24. When the grating structure 10 is used for photonic applications, the thickness of the base layer 24 may be varied as well to provide different performance characteristics.

[0046] The first functional layer 26 may further include nanogaps 32 as well, seen in FIGS. 3 and $\mathbf{4}$, depending on the angle of deposition of the plasmonic or photonic material
onto the base layer 24. If the plasmonic or photonic material onto the base layer $\mathbf{2 4}$. If the plasmonic or photonic material
of the first functional layer $\mathbf{2 6}$ is deposited onto the base layer 24 at an angle that is roughly normal to the plane of the substrate 12 and the base layer 24, as shown in FIG. 1, then no nanogaps $\mathbf{3 2}$ in the first functional layer $\mathbf{2 6}$ are formed. If the plasmonic or photonic material is deposited at an angle, such as 8 shown in FIG. 3, away from the normal, then nanogaps $\mathbf{3 2}$ may be formed in parallel with the grating elements $\mathbf{1 6}$. The deposition angle may range from approximately zero degrees to approximately 85 degrees from planar normal, with a range from approximately 65 degrees to approximately 80 degrees providing optimal results. Typically, the plasmonic or photoproviding optimal results. Typically, the plasmonic or photo-
nic material is also deposited in a direction that is transverse to the longitudinal axis of the grating elements $\mathbf{1 6}$.

[0047] When the first functional layer 26 is deposited at an angle other than planar normal, each grating element 16 may include three regions, as seen in FIGS. 3 and $\mathbf{4}$, instead of just the two discussed above. The regions may include the nanogap 32, a tip 34, and a plateau $\mathbf{3 6}$. Furthermore, the tip $\mathbf{3 4}$ may include a plurality of nanospurs $\mathbf{5 8}$. When viewed in may include a plurality of nanospurs $\mathbf{5 8}$. When viewed in
cross-sectional profile, the nanogap $\mathbf{3 2}$ may present a low cross-sectional profile, the nanogap 32 may present a low
point for the grating element 16, while the tip 34 may present a high point, with an angled, generally smooth surface therebetween. The plateau 36 may have a rounded shape that falls from the tip of one grating element 16 to the nanogap 32 of the the first functional layer 26 was deposited at an angle of approximately 76 degrees away from planar normal, the nanogap 32 has a width ranging from approximately $10 \mathrm{~nm}$ to approximately $30 \mathrm{~nm}$, the tip $\mathbf{3 4}$ has a width ranging from approximately $10 \mathrm{~nm}$ to approximately $30 \mathrm{~nm}$, and the plateau 36 has a width of approximately $90 \mathrm{~nm}$. The nanospurs $\mathbf{5 8}$ may include or may form a plurality of peaks abutting one Each nanospur $\mathbf{5 8}$ may have a shape of roughly a triangle, roughly a sawtooth, roughly a half sine wave, variations thereof, or combinations thereof. At the edges of each nanospur $\mathbf{5 8}$, or where the nanospurs $\mathbf{5 8}$ join with one another, there may be a sharp point.

[0048] The nanogap 32 and the tip 34 may behave like electromagnetic field concentrators, wherein the nanogap 32 acts as a lightning rod and the tip $\mathbf{3 4}$ acts as a nano antenna. The plateau 36 experiences interference from the two distinct electromagnetic fields resulting in either constructive or destructive interference. The large electromagnetic fields produced in the nanogap 30 , the tip $\mathbf{3 4}$, and the plateau 36 are a result of the localized electromagnetic field enhancement. When the grating structure $\mathbf{1 0}$ is utilized in fluorescence imaging, either the lightning rod, the nano antenna, or the constructive interference effect may cause any fluorophore placed in the right region to fluoresce with a several fold higher intensity in comparison to the other regions. In addition, the nanospurs $\mathbf{5 8}$ positioned along the tips $\mathbf{3 4}$ may enhance or amplify the electromagnetic field to create regions along the grating structure 10 with an increased electromagnetic field known as hotspots.

[0049] In various embodiments, the grating structure 10 may further include a second functional layer $\mathbf{6 0}$, as seen in FIGS. 5 and $\mathbf{6}$, positioned on the upper surface of the first functional layer 26. Generally, with such embodiments, the first functional layer $\mathbf{2 6}$ is a photonic material functional laye 26 wherein the photonic material is a dielectric such as titanium oxide. The first functional layer $\mathbf{2 6}$ is deposited at angle of approximately zero degrees from planar normal, so that the first functional layer $\mathbf{2 6}$ may have a shape as seen in FIG. 1. The second functional layer 60 typically includes plasmonic material such as silver that is deposited at an angle ranging from greater than zero degrees to approximately 85 degrees from planar normal. Alternatively, both the first functional layer $\mathbf{2 6}$ and the second functional layer $\mathbf{6 0}$ may include plasmonic materials. 
[0050] At least a portion of the steps of a method 100, in accordance with various aspects of the invention, for producing a grating structure $\mathbf{1 0}$ is shown in FIG. 7. The steps of the method $\mathbf{1 0 0}$ may be performed in the order as shown in FIG. 7 , or they may be performed in a different order. Furthermore, some steps may be performed concurrently as opposed to sequentially. In addition, some steps may not be performed. [0051] Referring to step 101, a stamp 38 is formed by applying a flexible plastic material to a mold $\mathbf{4 0}$, shown in FIG. 8, which includes a grating pattern $\mathbf{4 2}$ such that the plastic material receives and retains a negative of the grating pattern 42. The plastic material may include any flexible, somewhat resilient polymer that can adapt to the shape of a mold and retain that shape. An exemplary plastic material is polydimethylsiloxane (PDMS)

[0052] The mold $\mathbf{4 0}$ may be any solid object that includes the grating pattern $\mathbf{4 2}$ on one of its surfaces and may be constructed from materials such as metals, glass, silicon, or the like. The process described below or by forming the grating pattern $\mathbf{4 2}$ in the mold $\mathbf{4 0}$ material using patterning and etching, e-beam lithography, reactive ion etching, machining, or the like. An exemplary mold $\mathbf{4 0}$ is an optical disc, such as a compact disc (CD), a digital video disc (DVD), a high definition DVD (HD-DVD), a Blu-ray ${ }^{\mathrm{TM}}$ disc, etc., which includes an internal data layer defined by a grating pattern, the same as or similar to the grating pattern $\mathbf{4 2}$, with a portion of the disc removed to expose the data layer and the grating pattern, as seen in FIG.

[0053] The grating pattern $\mathbf{4 2}$ may be similar to the array 14, discussed above, wherein the grating pattern $\mathbf{4 2}$ includes a plurality of grating elements $\mathbf{1 6}$ with the characteristics mentioned above. The grating elements 16 of an exemplary grating patte 42 may include a longitudina peak 20 posigrating tioned next to a longitudinal valley $\mathbf{2 2}$ that in combination have a cross-sectional width of approximately $400 \mathrm{~nm}$. [0054] The plastic material that forms the stamp 38 may initially be in a liquid form and may be applied to the surface of the mold $\mathbf{4 0}$ that includes the grating pattern $\mathbf{4 2}$ by spin coating the plastic material onto the mold 40 in a known fashion, the result of which is shown in FIGS. 9 and 10. The plastic material may cure on the mold $\mathbf{4 0}$ until it hardens and is solid to the touch - thereby forming the stamp 38 .

[0055] Referring to step 102, the stamp 38, or a portion thereof, is removed from the mold $\mathbf{4 0}$ and is placed on a temporary substrate $\mathbf{4 4}$. The temporary substrate $\mathbf{4 4}$ is generally a rigid body with at least one surface that is flat and smooth An exemplary temporary substrate 44 is a glass slide, silich An exemp 38 is formed, seen in FIGS. 9-11, it may include a first surface 46 which includes the negative of the grating pattern $\mathbf{4 2}$ and an opposing second surface 48 which is generally flat and smooth. The stamp 38 is placed on the temporary substrate 44 such that the second surface 48 contacts the temporary substrate 44 and the first surface $\mathbf{4 6}$ is exposed and accessible, as seen in FIG. $\mathbf{1 2}$. [0056] Referring to step $\mathbf{1 0 3}$, an ink $\mathbf{5 0}$ is formed by applying a polymer film to the stamp $\mathbf{3 8}$ to receive and retain a positive of the grating pattern 42 . The polymer film may include any flexible, somewhat resilient polymer that can adapt to the shape of a mold and retain that shape. An exemplary polymer film is polymethylsilsesquioxane (PMSSQ). In various embodiments, the PMSSQ may be mixed with ethanol. The polymer film that forms the ink $\mathbf{5 0}$ may initially be in a liquid form and may be applied to the stamp $\mathbf{3 8}$ by spin coating onto the exposed first surface 46, the result of which is shown in FIGS. 13 and 14. The polymer film may cure on the stamp 38 until it hardens and is solid to the touchthereby forming the ink $\mathbf{5 0}$. After its formation, the ink $\mathbf{5 0}$ may include a first surface $\mathbf{5 2}$ and an opposing second surface $\mathbf{5 4}$, as best seen in FIG. 14. The first surface 52 includes the positive of the grating pattern $\mathbf{4 2}$ and is in contact with the first surface $\mathbf{4 6}$ of the stamp 38 . The second surface 54 is generally flat and smooth

[0057] Referring to step 104, nanogaps 30 are created in the ink 50, similar to those of the base layer 24 seen in FIG. 2 . The nanogaps $\mathbf{3 0}$ may include tears, openings, or gaps in the polymer material of the ink $\mathbf{5 0}$. Exemplary nanogaps $\mathbf{3 0}$ may polymer material of the ink $\mathbf{5 0}$. Exemplary nanogaps $\mathbf{3 0}$ may
have a width ranging from approximately $20 \mathrm{~nm}$ to approximately $30 \mathrm{~nm}$, while the length of the nanogaps 30 may vary more greatly. The nanogaps $\mathbf{3 0}$ may be formed by applying a tensional force to opposing ends of the stamp $\mathbf{3 8}$ and the ink 50 while they are in contact with one another. The nanogaps 30 may also be formed by poking holes in the combination of the stamp 38 and the ink $\mathbf{5 0}$, by bending the combination of the stamp $\mathbf{3 8}$ and the ink $\mathbf{5 0}$, or by reverse bending the combination of the stamp $\mathbf{3 8}$ and the ink $\mathbf{5 0}$. This step may be
optional, it may be performed after the stamp $\mathbf{3 8}$ and ink $\mathbf{5 0}$ optional, it may be performed after the stamp 38 and ink 50 performed during or after step $\mathbf{1 0 5}$.

[0058] Referring to step 105, the stamp 38 and the ink $\mathbf{5 0}$ are removed from the temporary substrate $\mathbf{4 4}$ and placed on a permanent substrate 56, as shown in FIGS. 15 and 16. The permanent substrate $\mathbf{5 6}$ may be similar to the temporary substrate 44. An exemplary permanent substrate $\mathbf{5 6}$ is a glass slide, a silicon wafer, or the like. The stamp $\mathbf{3 8}$ and ink $\mathbf{5 0}$ may be removed from the temporary substrate $\mathbf{4 4}$ as a unit while still contacting one another. They may be removed manually by a technician or by an automated machine. The stamp $\mathbf{3 8}$ and ink $\mathbf{5 0}$ may be placed on the permanent substrate $\mathbf{5 6}$ with the second surface of the ink $\mathbf{5 0}$ contacting a surface of the permanent substrate 56 and the second surface of the stamp 38 facing away from the permanent substrate 56 . The placement may be performed manually or by automated machine. After the placement of the stamp 38 and ink 50, a period of time may elapse to allow the ink $\mathbf{5 0}$ to seal with the permanent substrate 56.

[0059] Referring to step 106, the stamp 38 is removed from the ink $\mathbf{5 0}$. In some embodiments, the stamp $\mathbf{3 8}$ may be peeled from the ink $\mathbf{5 0}$ by manually lifting one corner or edge of the stamp 38, as seen in FIG. 17, with a pair of tweezers. In other embodiments, the stamp $\mathbf{3 8}$ may be removed from the ink $\mathbf{5 0}$ by an automated machine. The process of removing the stamp $\mathbf{3 8}$ from the ink $\mathbf{5 0}$ may create nanogaps $\mathbf{3 0}$ in the stamp $\mathbf{3 8}$ which are transferred to the ink $\mathbf{5 0}$. Thus, after removing the stamp $\mathbf{3 8}$ from the ink $\mathbf{5 0}$, the ink $\mathbf{5 0}$ may a plurality of nanogaps 30 that are randomly oriented and generally positioned in proximity to the perimeter of the ink 50. Furthermore, after removing the stamp $\mathbf{3 8}$, the first surface $\mathbf{5 2}$ of the ink 50 is exposed such that the positive of the grating pattern $\mathbf{4 2}$ is facing upwards. In addition, the ink 50 on the permanent substrate 56 generally forms the base layer $\mathbf{2 4}$ on the substrate 12 of the grating structure 10

[0060] Referring to step 107, a first functional layer 26 is applied to the ink $\mathbf{5 0}$. The first functional layer 26 is applied to the first surface $\mathbf{5 2}$ on top of the grating pattern $\mathbf{4 2}$. The first functional layer $\mathbf{2 6}$ may include plasmonic material, such as metals, or photonic material, such as dielectrics, as discussed above. The first functional layer $\mathbf{2 6}$ may be applied to the ink 
50 using known deposition techniques. The first functional layer $\mathbf{2 6}$ may also be applied to the ink $\mathbf{5 0}$ by chemical procedures such as the sol-gel process in which the material of the first functional layer $\mathbf{2 6}$ is applied to the ink $\mathbf{5 0}$ in a solution. In some embodiments, the material of the first functional layer 26 may deposited at an angle that is normal to the plane of the ink 50. The result of the deposition may be similar to the grating structure 10 as shown in FIGS. 1 and $\mathbf{3}$. The first functional layer $\mathbf{2 6}$ may also be applied such that it does not fill in or completely cover the nanogaps 30 which are formed in the ink $\mathbf{5 0}$.

[0061] In other embodiments, the material of the first functional layer 26 may deposited at an angle that is not normal to the plane of the ink $\mathbf{5 0}$, similar to the deposition illustrated in FIG. 3. This angled deposition is typically accomplished by tilting the permanent substrate $\mathbf{5 6}$ and the ink $\mathbf{5 0}$ thereupon with respect to the source from which the first functional layer $\mathbf{2 6}$ material is deposited. The deposition angle, i.e., the angle of tilt, may range from approximately zero degrees to approximately 85 degrees from planar normal. Furthermore, the axis of tilt for the permanent substrate $\mathbf{5 6}$ is generally parallel to the longitudinal axis of the grating elements 16 of the grating pattern $\mathbf{4 2}$ of the ink 50 . The tilting of the permanent substrate 56 and the ink 50 during deposition of the first functional layer $\mathbf{2 6}$ may result in each grating element $\mathbf{1 6}$ having the features of the nanogap $\mathbf{3 2}$, the tip $\mathbf{3 4}$, the plateau $\mathbf{3 6}$, and the nanospurs $\mathbf{5 8}$, as described above and shown in FIGS. 3 and 4.

[0062] The permanent substrate 56 and the ink 50 may have an inherent surface energy or their surface energies may be controlled and adjusted. The material of the first functional layer 26 may have an inherent energy level or may be given an energy level as well. In addition, the material of the first material of the first functional layer $\mathbf{2 6}$, the permanent substrate 56, and the ink $\mathbf{5 0}$ may be controlled or adjusted thermally, through temperature control, electrically, through voltage control, or by other methods. The energy levels of one or more of the three components may determine the optimal angle for applying the material of the first functional layer $\mathbf{2 6}$ in order to form the features of the nanogap 32 , the tin 34 , the plateau 36 , and the nan as the spacing between grating elements 16, the height of the peak 20 , and/or the aspect ratio of the height to the spacing may also influence the value of the optimal angle for applying the material of the first functional layer 26. Furthermore, the thickness of the functional layer $\mathbf{2 6}$ may influence the value of the optimal angle for applying the material of the first functional layer 26. As an example, for an ink $\mathbf{5 0}$ made from PMSSQ with a grating pattern $\mathbf{4 2}$ that is formed from an HD-DVD mold $\mathbf{4 0}$ (and has the corresponding spacings and heights) and a first functional layer 26 of silver applied with a thickness of $40 \mathrm{~nm}$, the optimal angle of deposition is approximately 75 degrees. [0063] In certain embodiments, the first functional layer $\mathbf{2 6}$ may include one or more sublayers of material. For example, when forming a plasmonic first functional layer $\mathbf{2 6}$, there may be a sublayer of metal, which enhances adhesion between the ink $\mathbf{5 0}$ and the first functional layer $\mathbf{2 6}$. The sublayers may be
deposited onto the ink $\mathbf{5 0}$ in the same fashion as the first deposited onto the
functional layer $\mathbf{2 6}$

[0064] Referring to step 108, a second functional layer 60 is applied to the first functional layer 26, as shown in FIGS. 5 and 6. This step may be optional. However, when it is per- formed, the first functional layer 26 typically includes photonic material such as titanium oxide, and the second functional layer 60 includes plasmonic material such as silver. Furthermore, the first functional layer $\mathbf{2 6}$ is deposited at angle of approximately zero degrees from planar normal. The second functional layer 60 is typically deposited at an angle ond functional layer 60 is typically deposited at an angle
ranging from greater than zero degrees to approximately 85 degrees from planar normal.

[0065] At least a portion of the steps of a method 200, in accordance with various aspects of the invention, for producing a mold $\mathbf{4 0}$ with a grating pattern $\mathbf{4 2}$ is shown in FIG. 18. The steps of the method $\mathbf{2 0 0}$ may be performed in the order as shown in FIG. 18, or they may be performed in a different order. Furthermore, some steps may be performed concurrently as opposed to sequentially. In addition, some steps may not be performed.

[0066] Referring to step 201, an optical disc 300 is obtained, including a label side 302, a transparent side 304, and a metal layer 306, as seen in FIGS. 19 and 21. The optical disc 300 may include a perimeter ring 308 and a center ring $\mathbf{3 1 0}$ as well. The optical disc $\mathbf{3 0 0}$ may be of known optical $\mathbf{3 1 0}$ as well. The optical disc $\mathbf{3 0 0}$ may be of known optical
data storage disc types with data tracks arranged in concentric circles. Exemplary optical dises 300 include 12-centimeter diameter dises, such as compact disc (CD), digital video disc (DVD), high definition DVD (HD-DVD), Blu-ray ${ }^{\mathrm{TM}}$ disc, and the like. Typically, the optical disc $\mathbf{3 0 0}$ is blank (with no pre-recorded data on it), single sided, and with a single data layer. The label side $\mathbf{3 0 2}$ may be what is considered the top surface of the disc, often with a manufacturer's name or logo on it. The label side 302 may also have an opaque coating. The transparent side 304 may be considered the bottom surface of the disc, on the opposite side from the label. The label side 302 and the transparent side 304 may both include a rigid polymer material, such as polycarbonate, that gives the optical disc 300 its structure. During usage of the optical disc $\mathbf{3 0 0}$, a laser shines light through the transparent side 304 in order to read data from the optical disc $\mathbf{3 0 0}$. Thus, the transparent side 304 is transmissive to light of varying wavelengths. The metal layer 306 generally provides reflection of the laser light which determines the data stored on the optical disc $\mathbf{3 0 0}$. The perimeter ring 308 is a space at the edge of the optical disc 300 in which there are no data tracks.

[0067] At the metal layer 306, there may be two grating patterns forming the data tracks, one on the label side 302 and one on the transparent side 304, wherein one grating pattern is a mirror image or negative of the other and the metal layer is positioned between the two patterns. Furthermore, the grating patterns may be similar to the grating pattern $\mathbf{4 2}$ and the array 14 of grating elements 16.

[0068] Referring to step 202, a position of the metal layer 306 is determined with respect to the label side 302 and the transparent side 304. The thickness of the optical disc 300 is generally constant for the different types of discs, however the position of the metal layer 306 varies with the type. The metal layer 306 in a $C D$ is close to the label side 302 . The metal layer 306 in a DVD and an HD-DVD is positioned near the center of the optical disc 300. In a Blu-ray ${ }^{\mathrm{TM}}$ disc, the metal layer 306 is close to the transparent side $\mathbf{3 0 4}$.

[0069] Referring to step 203, the optical disc 300 is split at the metal layer 306 along the plane of the disc from the outer edge at the perimeter ring 308 toward the center ring 310 , if the metal layer 306 is closer to the transparent side 304 than the label side $\mathbf{3 0 2}$ as seen in FIG. 19. The optical disc 300 is likely a Blu-ray ${ }^{\mathrm{TM}}$ disc. An exemplary method to splitting the 
optical disc 300 includes cutting the optical disc $\mathbf{3 0 0}$ with a sharp object, such as a razor blade, at the edge of the disc parallel to its plane. The optical disc $\mathbf{3 0 0}$ usually separates into two pieces along the metal layer 306 the two pieces being the label side 302 and the transparent side 304 .

[0070] Referring to step 204, the transparent side 304 and the metal layer $\mathbf{3 0 6}$ are removed from the optical disc $\mathbf{3 0 0}$. Thus, the label side $\mathbf{3 0 2}$ with a grating pattern remain, as seen in FIG. 20. In some cases, fragments of the metal layer 306 may remain as well.

[0071] Referring to step 205, the optical disc 300 is placed in a solvent to remove any remaining metal. An exemplary solvent includes $15 \%$ nitric acid. Other solvents, cleaners, and debris removal techniques may be utilized as well. [0072] Referring to step 206, the perimeter ring 308 is removed from the optical disc $\mathbf{3 0 0}$, if the metal layer $\mathbf{3 0 6}$ is not closer to the transparent side 304 than the label side $\mathbf{3 0 2}$, as seen in FIG. 21. The optical disc $\mathbf{3 0 0}$ may be a DVD or an HD-DVD. The perimeter ring 308 may be removed by cutting it off of the optical disc $\mathbf{3 0 0}$ using a sharp object or by grinding it off. Removal of the perimeter ring $\mathbf{3 0 8}$ may make it easier to access the metal layer 306 for performing the next step.

[0073] Referring to step 207, the optical disc 300 is split at the metal layer 306 along the plane of the disc from the outer edge toward the center ring 310 . As discussed in step 203, the optical disc $\mathbf{3 0 0}$ separates into two pieces, the label side $\mathbf{3 0 2}$ and the transparent side 304, along the metal layer 306.

[0074] Referring to step 208, the label side 302 and the metal layer 306 are removed from the optical disc 300 . Thus, the transparent side 304 with a grating pattern remain, as seen in FIG. 22. In some cases, fragments of the metal layer $\mathbf{3 0 6}$ may remain as well.

[0075] Referring to step 209, the optical disc 300 is placed in a solvent to remove any remaining metal. As with step 205, the solvent may include $15 \%$ nitric acid. Other solvents, cleaners, and debris removal techniques may be utilized as well.

[0076] Although the invention has been described with reference to the embodiments illustrated in the attached drawing figures, it is noted that equivalents may be employed and substitutions made herein without departing from the scope of the invention as recited in the claims.

Having thus described various embodiments of the invention, what is claimed as new and desired to be protected by Letters Patent includes the fo

1. A grating structure comprising:

a substrate including a smooth upper surface;

a base layer positioned on the substrate, the base layer including

a first surface with a plurality of grating elements positioned adjacent one another, each grating element including a longitudinal peak and a longitudinal valley, and

a second surface, opposing the first surface, in contact with the upper surface of the substrate; and

a first functional layer for providing electromagnetic field enhancement in the vicinity of the grating structure, the functional layer positioned on the second surface of the base layer.

2. The grating structure of claim 1 , wherein the first functional layer includes a longitudinal peak aligned with the peak of the base layer and a longitudinal valley aligned with the valley of the base layer.
3. The grating structure of claim 1 , wherein the first functional layer includes a nanogap, a plateau, and a tip, wherein the nanogap is a longitudinal valley, the tip is a longitudinal peak, and the plateau is a rounded surface extending therebetween.

4. The grating structure of claim 3 , wherein the tip includes a plurality of nanospurs abutting one another along the length thereof, wherein each nanospur includes or forms a peak.

5 . The grating structure of claim 1 , further comprising a second functional layer applied to an upper surface of the first functional layer, such that the first functional layer is formed from a dielectric and the second functional layer is formed from a metal or a metal alloy.

6. The grating structure of claim 1 , wherein the first functional layer is formed from a metal or a metal alloy.

7. The grating structure of claim 1 , wherein the first functional layer is formed from a dielectric.

8. A method of producing a grating structure, the method comprising the steps of:

forming a stamp from flexible plastic material, the stamp including a negative of a periodic grating pattern on a first surface;

forming an ink by applying a polymer film to the stamp, the ink including a first surface and an opposing second surface, wherein the first surface of the ink contacts the first surface of the stamp such that the ink retains a positive of the periodic grating pattern;

placing the ink and the stamp on a substrate such that the second surface of the ink contacts an upper surface of the substrate; and

removing the stamp from the ink by applying a tensional force to one edge of the stamp.

9. The method of claim $\mathbf{8}$, further comprising the step of applying a first functional layer to the first surface of the ink.

10. The method of claim 9 , wherein the functional layer includes a metal or a metal alloy.

11. The method of claim 9 , further comprising the step of applying a second functional layer to an upper surface of the first functional layer

12. The method of claim 11, wherein the ink includes a plane and the first functional layer is deposited onto the ink approximately normal to the plane of the ink and the second functional layer is deposited onto the first functional layer at an angle away from the plane of the ink.

13. The method of claim 9 , wherein the ink includes a plane and the first functional layer is deposited onto the ink at an angle away from normal to the plane of the ink.

14. The method of claim 13, wherein the angle ranges from greater than 0 degrees to approximately 85 degrees.

15. The method of claim 8 , further comprising the step of applying a flexible plastic material to a mold, which includes the grating pattern, to form the stamp.

16. The method of claim 15 , wherein the mold includes an optical disc with an internal grating pattern, wherein a portion of the optical disc is removed to expose the grating pattern.

17. A method of producing a grating structure, the method comprising the steps of:

forming a stamp by applying a flexible plastic material to a mold which includes a periodic grating pattern, wherein the stamp retains a negative of the grating pattern on a first surface;

forming an ink by applying a polymer film to the stamp, the ink including a first surface and an opposing second surface, wherein the first surface of the ink contacts the 
first surface of the stamp such that the ink retains a positive of the periodic grating pattern;

placing the ink and the stamp on a substrate such that the second surface of the ink contacts an upper surface of the substrate;

substrate;
removing the stamp from the ink by applying a tensional force to one edge of the stamp; and

applying a functional layer to the first surface of the ink.

18. The method of claim 17, further comprising the step of applying a second functional layer to an upper surface of the first functional layer.

19. The method of claim 17, wherein the ink includes a

19. The the first function plane and he first functional layer is deposited onto the ink approximately normal to the plane of the ink and the second functional layer is deposited onto the first functional layer at an angle away from the plane of the ink.

20. The method of claim 17, wherein the mold includes an optical disc with an internal grating pattern, wherein a portion of the optical disc is removed to expose the grating pattern.

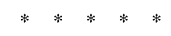




\subsubsection{VITA}

Born 1987, Drew Edwin Menke's early childhood was spent mostly in Illinois, but he later moved to Missouri where he graduated from New Haven high school. In 2010 he graduated with his Bachelor of Science degree in Biological Sciences, with a minor in chemistry, from the Missouri University of Science and Technology. He continued his education in the Biochemistry department at the University of Missouri, Columbia, where he will be receiving his $\mathrm{Ph} . \mathrm{D}$. this fall 2017. His future plans are to enjoy life. 